\title{
Mikrophasenseparation von photo-vernetzbaren Blockcopolymeren in dünnen Filmen
}

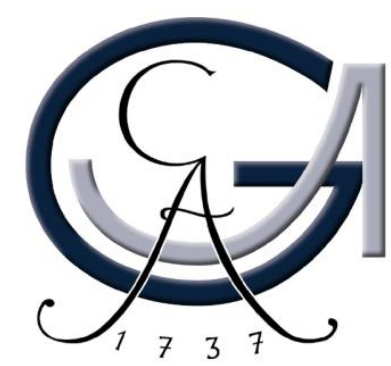

Dissertation

zur Erlangung des mathematisch-naturwissenschaftlichen Doktorgrades

„Doctor rerum naturalium“

an der Georg-August-Universität Göttingen

im Promotionsprogramm Chemie

der Georg-August University School of Science (GAUSS)

vorgelegt von

Katharina Tietz

aus Blankenburg

Göttingen 2014 
Betreuungsausschuss

Prof. Dr. Philipp Vana Makromolekulare Chemie, Institut für Physikalische Chemie, Georg-August-Universität Göttingen

Prof. Dr. Michael Buback Technische und Makromolekulare Chemie, Institut für Physikalische Chemie, Georg-August-Universität Göttingen

\section{Mitglieder der Prüfungskommission}

Referent:

Prof. Dr. Philipp Vana $\quad$ Makromolekulare Chemie, Institut für Physikalische Chemie, Georg-August-Universität Göttingen

Korreferent:

Prof. Dr. Michael Buback Technische und Makromolekulare Chemie, Institut für Physikalische Chemie, Georg-August-Universität Göttingen

Weitere Mitglieder der Prüfungskommission

Prof. Dr. Peter Botschwina Theoretische Chemie, Institut für Physikalische Chemie, Georg-August-Universität Göttingen

Prof. Dr. Burkhard Geil Biophysikalische Chemie, Institut für Physikalische Chemie, Georg-August-Universität Göttingen

Jun.-Prof. Dr. Ricardo Mata Computerchemie und Biochemie, Institut für Physikalische Chemie, Georg-August-Universität Göttingen

PD Dr. Thomas Zeuch Physikalische Chemie II, Institut für Physikalische Chemie, Georg-August-Universität Göttingen

Tag der mündlichen Prüfung: 15.12 .2014 
Meinen Eltern 


\section{Inhaltsverzeichnis}

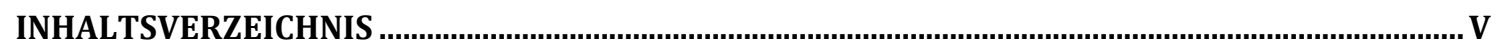

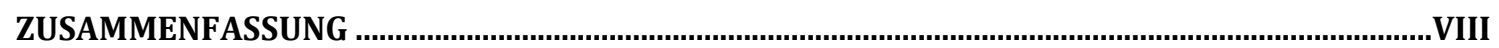

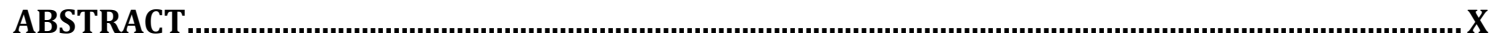

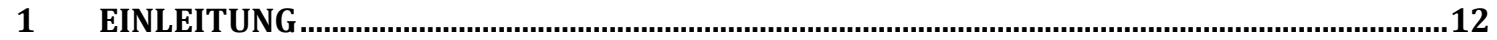

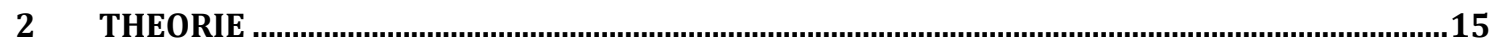

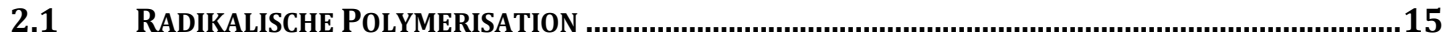

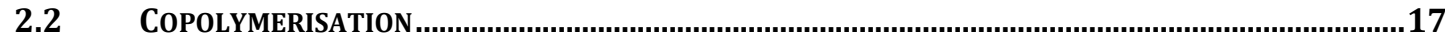

2.3 REVERSIBEL-DESAKTIVIERENDE RADIKALISCHE POLYMERISATION.............................................17

2.3.1 Reversibler Additions-Fragmentierungs-Kettentransfer (RAFT) ..................................19

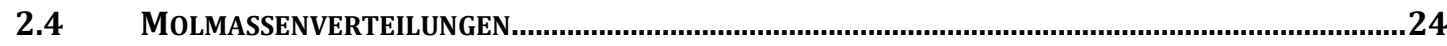

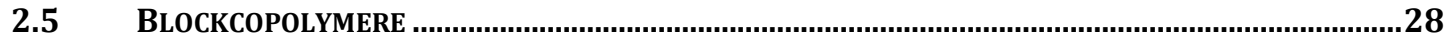

2.5.1 Mikrophasenseparation in Blockcopolymeren ....................................................................30

2.5.2 Morphologien der Mikrophasenseparation........................................................................33

2.5.3 Weitere Einflussfaktoren bei der Mikrophasenseparation ..................................................36

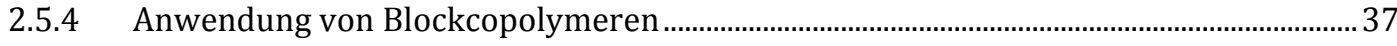

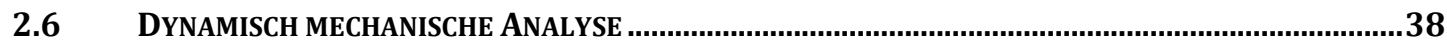

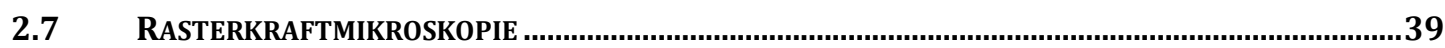

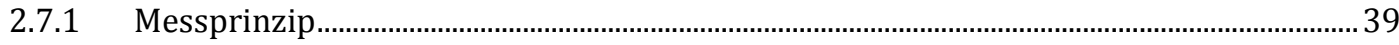

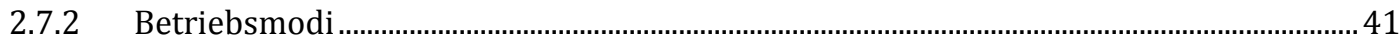

2.7.3 Bestimmung mechanischer Eigenschaften mittels AFM ................................................ 42

2.7.4 Bildanalyse mit Hilfe der Leistungsspektraldichte-Funktion............................................ 45

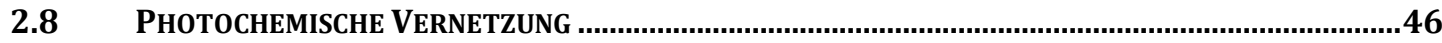

2.8.1 Coumarin und seine Anwendungsgebiete.............................................................................. 47

2.8.2 Photodimerisierung und Dimerspaltung ........................................................................... 47

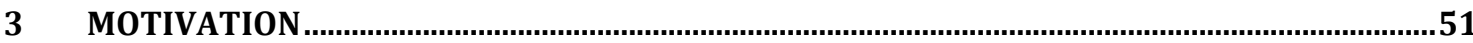

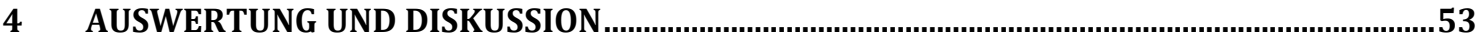

4.1 COPOLYMERISATION DER COUMARIN-DERIVATE .......................................................................53

4.1.1 Copolymerisation von MMA mit CMA …................................................................................ 54

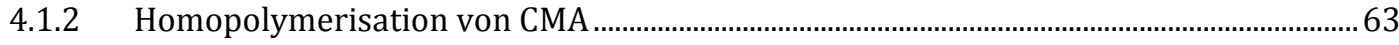

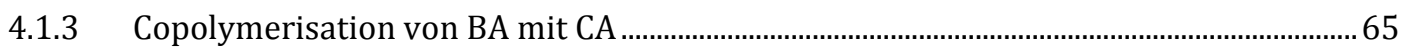

4.1.4 Übersicht verwendeter makroRAFT-Agenzien.......................................................................... 70 


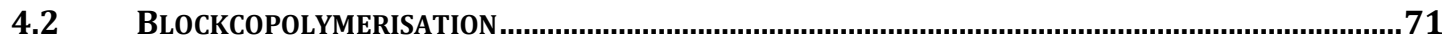

4.2.1 Herstellung von P(MMA-co-CMA)- $b$-PBA .............................................................................. 72

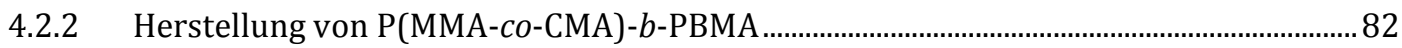

4.2.3 Übersicht verwendeter Blockcopolymere ...........................................................................87

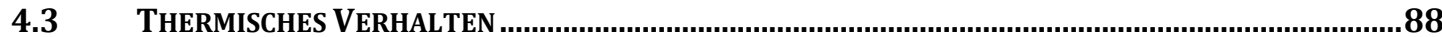

4.4 BESTIMMUNG DER MIKROPHASENSEPARATION DER BLOCKCOPOLYMERSYSTEME MITTELS AFM.93

4.4.1 Untersuchung des Blockcopolymersystems P(MMA-co-CMA)- $b$-PBA .......................... 93

4.4.2 Untersuchung des Blockcopolymersystems P(MMA-co-CMA)- $b$-PBMA ....................... 106

4.5 EINFLUSS DER TEMPERBEDINGUNGEN AUF DIE MIKROPHASENSEPARATION................................. 109

4.5.1 Defektdefinition für Probe P(MMA95-co-CMA9)- $b-\mathrm{PBA}_{229}$ [VII] ...................................... 111

4.5.2 Abhängigkeit von der Temperzeit .............................................................................................. 112

4.5.3 Abhängigkeit von der Tempertemperatur ........................................................................... 117

4.5.4 Defektentwicklung in Abhängigkeit von der Temperzeit und Temperatur .................. 118

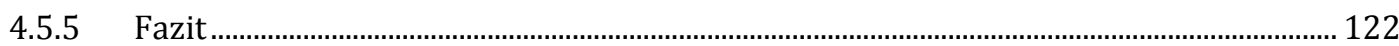

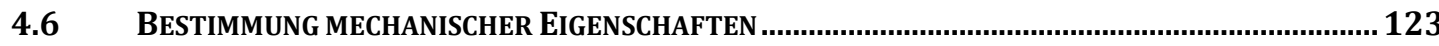

4.6.1 Verwendung von PeakForce-QNM ${ }^{\mathrm{TM}}$ bei P(MMA390-Co-CMA 29$)-b-\mathrm{PBA}_{426} \ldots \ldots \ldots . . . . . . . . . . . . . . .123$

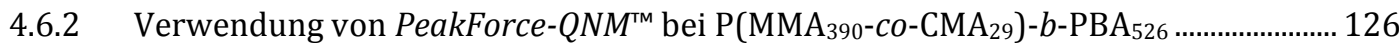

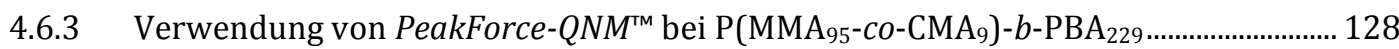

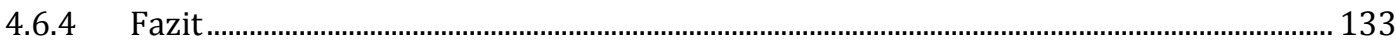

4.7 UV-INDUZIERTE DIMERISIERUNGSREAKTION UND DIMERSPALTUNG .......................................... 134

4.7.1 Dimerisierung der Coumarin-Einheiten des BCPs P(MMA-co-CMA)- $b$-PBA ................ 135

4.7.2 Dimerspaltung der Coumarin-Einheiten des BCPs P(MMA-co-CMA)- $b$-PBA ............... 141

4.8 ERMITTLUNG DES ORDNUNGS-UNORDNUNGS-ÜBERGANGS................................................... 145

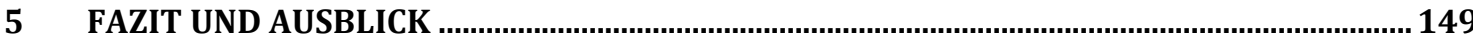

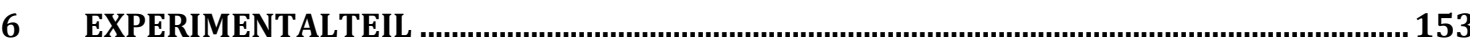

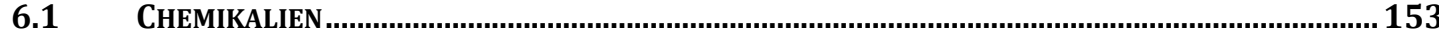

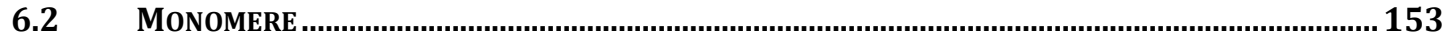

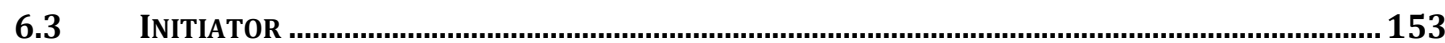

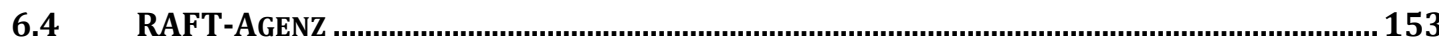

6.5 SPEKTROSKOPISCHE UND ANALYTISCHE METHODEN ............................................................ 154

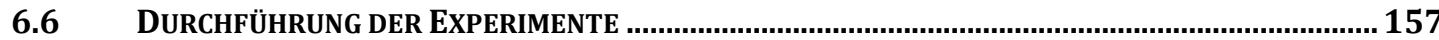

6.6.1 Synthese von 7-(2-Methacryloyloxyethoxy)-4-methylcoumarin (CMA) und 7-

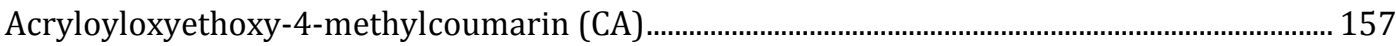

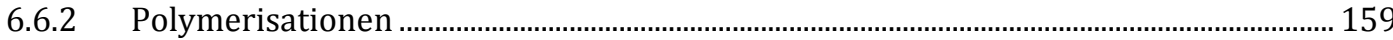

6.6.3 Probenpräparation, Messprobleme und Kalibrierung für AFM ..................................... 160

6.6.4 Probenpräparation für UV/Vis....................................................................................................... 164 
6.6.5 Durchführung der DMA-Experimente....................................................................................... 164

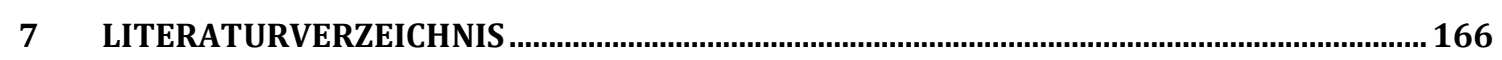

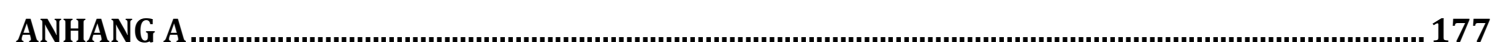

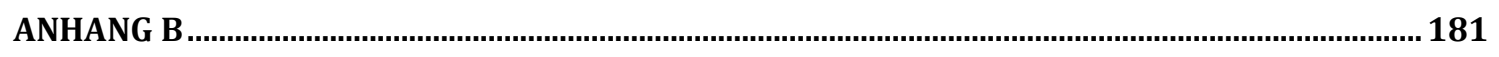

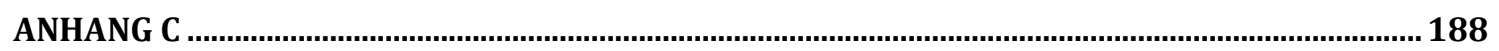

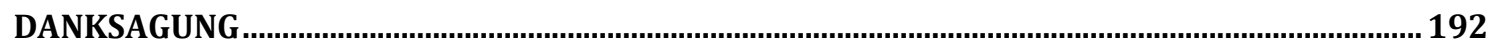

LEBENSLAUF ....................................................................FEHLER! TEXTMARKE NICHT DEFINIERT. 


\section{Zusammenfassung}

Im Rahmen dieser Arbeit wurden sowohl das Mikrophasenverhalten von Diblockcopolymeren mit eingebauten UV-vernetzbaren Einheiten mittels Rasterkraftmikroskopie (AFM) als auch deren mechanische Eigenschaften und der Einfluss der Vernetzungen auf die Selbstorganisation in Blockcopolymeren untersucht.

Die phasenseparierenden Blockcopolymersysteme wurden mittels der Reversible Addition-Fragmentation chain Transfer (RAFT)-Polymerisation synthetisiert, wobei Coumarin-Derivate als UV-vernetzbare Einheiten in eine Komponente des Blockcopolymers eingebaut wurden. Bei der zunächst durchgeführten Untersuchung der RAFT-Copolymerisation von Coumarin-Derivaten und verschiedenen Monomeren stellte sich die Kombination von Methylmethacrylat (MMA) und 7-(2-Methacryloyloxyethoxy)-4-methylcoumarin (CMA) aufgrund der Molmassenkontrolle als erfolgsversprechendste heraus. Zur Begünstigung der Mikrophasenseparation (MPS) wurde das erhaltene Copolymer (makroRAFTAgens) mit $n$-Butylacrylat (BA) bzw. Butylmethacrylat (BMA) für die Synthese von Blockcopolymersystemen mit Blöcken gegensätzlicher Eigenschaften polymerisiert, wobei die jeweiligen Blocklängen und Molmassen variiert wurden.

Die MPS des Blockcopolymersystems wurde mittels AFM dargestellt, wobei die vielfältige Morphologie wie z. B. Sphären, Zylinder und perforierte Lamellen von der Zusammensetzung der BCP abhängig ist. Der für die Ausbildung der Gleichgewichtsmorphologie entscheidende Temperprozess dauert bei einer Temperatur von $160^{\circ} \mathrm{C}$ mindestens $12 \mathrm{~h}$. Ein weiteres Indiz für die Phasenseparation der Polymere liefern die Ergebnisse der dynamisch mechanischen Analyse (DMA), womit zwei Glasübergangstemperaturen für die jeweiligen Blockcopolymere bestimmt wurden. Für die mittels AFM und DMA bestimmten lokalen und globalen Elastizitätsmoduln wurde eine gute Übereinstimmung festgestellt. Die Untersuchung der MPS der Blockcopolymere mit größeren Molmassen mittels in-situ-AFM-Messungen bei Temperaturen bis zu $210^{\circ} \mathrm{C}$ zeigte einen stabilen phasenseparierten Zustand des Systems. Dagegen wurde bei Blockcopolymeren mit kleineren Molmassen und gleicher Zusammensetzung ein Ordnungs-Unordnungs-Übergang bei Temperaturen $T>140^{\circ} \mathrm{C}$ festgestellt.

Die reversible Vernetzung der Blockcopolymere erfolgt durch die UV-Bestrahlung der Coumarin-Einheiten, die daraufhin eine [2+2]-Cycloaddition eingehen. Die 
erfolgreiche Dimerisierung und Dimerspaltung wurde mittels AFM und UV/VisSpektroskopie nachgewiesen, wobei bei der UV-Bestrahlung der Blockcopolymere eine hohe Effizienz für beide Reaktionen bei einer Temperatur von $125{ }^{\circ} \mathrm{C}$ festgestellt wurde. Die Vernetzung der Blockcopolymere führt zu einer Zunahme der Elastizitätsmoduln mit zunehmender Bestrahlungszeit. Außerdem konnte gezeigt werden, dass die Selbstorganisation in den Blockcopolymeren durch die Vernetzungsreaktion verhindert wird und dadurch das System in einen Nichtgleichgewichtszustand versetzt werden kann. 


\section{Abstract}

The micro-phase behavior of diblock copolymers with integrated UV-crosslinkable units has been investigated by atomic force microscopy (AFM) as well as their mechanical properties. Further, the influence of crosslinkers in the self-assembly in block copolymers was studied.

The phase separating block copolymer systems were synthesized by reversible addition-fragmentation chain transfer (RAFT) polymerization. One component of the block copolymers were decorated with coumarin derivatives as UV-crosslinkable units. In the first investigation of RAFT copolymerization of coumarin derivatives and various monomers, the combination of methyl methacrylate (MMA) and 7-(2-methacryloyloxyethoxy)-4-methylcoumarin (CMA) turned out to be the most successful type due to the molar mass control. To favor the micro-phase separation (MPS), the resulting copolymer (macroRAFT-agent) was copolymerized with $n$-butylacrylate (BA) or butyl methacrylate (BMA) to synthesize block copolymer systems consisting of blocks with contrarily properties and also varying block lengths and molecular weights.

The micro-phase separation of block copolymer systems was investigated by AFM. The versatile morphologies like spheres, cylinders and perforated lamellas depend on the composition of the block copolymers. The deciding annealing process for the formation of the equilibrium morphology takes at least $12 \mathrm{~h}$ at a temperature of $160^{\circ} \mathrm{C}$. The results of dynamic mechanical analysis (DMA) provide further indication for phase separation of the polymers whereby for the respective block copolymers two glass transition temperatures were determined. For the local and global elastic moduli, which were determined by AFM and DMA, a good agreement was found. The investigation of the MPS of the block copolymers with larger molecular weights by in situ AFM measurements at temperatures up to $210{ }^{\circ} \mathrm{C}$ showed a stable phase separated state of the system. In contrast, for the block copolymers with smaller molecular weights and same composition an order-disorder transition at temperatures $T>140{ }^{\circ} \mathrm{C}$ has been found.

The reversible crosslinking of the block copolymers is carried out by UV irradiation of the incorporated coumarin units that undergo a [2+2]-cycloaddition. The successful dimerization and cleavage was investigated by AFM and UV/Vis spectroscopy. The UV irradiation of the block copolymers showed a high efficiency for both reactions at a temperature of $125^{\circ} \mathrm{C}$. The crosslinking of the block 
copolymers leads to an increase in the moduli of elasticity with increasing irradiation time. Additionally, it could be shown that the self-assembly is prevented in the block copolymers by the crosslinking reaction and thereby the system can be transferred in a non-equilibrium state. 


\section{Einleitung}

Seit Beginn des 20. Jahrhunderts haben die Entwicklung und der Einsatz synthetischer Kunststoffe mit ihren vielfältigen Eigenschaften zu einer wertvollen Ergänzung herkömmlicher Werkstoffe wie Stahl, Holz, Keramik, Glas, Papier und Baumwolle geführt. Seit den 50er Jahren des letzten Jahrhunderts ist die Produktion weltweit von 1,7 Mio. Tonnen Kunststoffe auf 288 Mio. Tonnen im Jahr 2012 gestiegen. ${ }^{1}$ Kunststoffe finden heutzutage größtenteils Verwendung in der Verpackungsindustrie, im Baugewerbe sowie in der Fahrzeug- oder Elektroindustrie. Weitere wichtige Anwendungsgebiete für Kunststoffe sind Sport, Freizeit, Möbel, Haushaltsgegenstände und Medizin. ${ }^{1}$ Die Herstellung neuartiger Kunststoffe, die ihren Vorgängern in Eigenschaften, Anwendbarkeit und Funktionalität überlegen sind, erfordert intensive Forschung und Weiterentwicklung sowohl in der Industrie als auch in der Wissenschaft.

Die radikalische Polymerisation ist die bedeutendste Methode für die großtechnische Produktion von Kunststoffen, da sich mit ihr eine Vielzahl verschiedenster Monomere in unterschiedlichsten Polymerarchitekturen herstellen lassen. Dabei sind geringe Anforderungen an die Reinheit der Monomere und Lösungsmittel gestellt und die einfache Reaktionsführung ermöglicht die schnelle und kostengünstige Herstellung von einer Vielzahl von unterschiedlichsten Polymeren. ${ }^{2}$ In den 1920er Jahren legte Staudinger mit seinen Theorien über den Aufbau von Kunststoffen die Grundlagen für die Erforschung der radikalischen Polymerisation. ${ }^{3-5}$ Von diesem Zeitpunkt an wurden auf dem Gebiet der Polymerforschung viele wichtige Fortschritte erzielt. Aufgrund der zahlreichen Vorteile dieser Polymerisationstechnik betrug der Anteil der mittels radikalischer Polymerisation produzierten Kunststoffe im Jahr 2009 etwa $45 \%$ der weltweiten Gesamtproduktion von Polymeren und $40 \%$ bei synthetischem Gummi. ${ }^{6}$

Heutzutage werden immer höhere Anforderungen an die Kunststoffe gestellt und stetig werden neue Anwendungsgebiete wie z. B. in der Nanotechnologie oder in der Medizin erschlossen.7,8 Der gezielte Aufbau von Polymeren und die exakte Kontrolle der molekularen Architektur wie Zusammensetzung, Topologie und Funktionalität (siehe Abbildung 1-1) hat damit eine steigende Bedeutung erfahren. ${ }^{9}$ Für die Herstellung neuartiger Materialien sind genaue Kenntnisse der 
Struktur-Eigenschaftsbeziehungen notwendig sowie Polymerisationsverfahren, die eine exakte Kontrolle der molekularen Architektur eines Polymers ermöglichen.

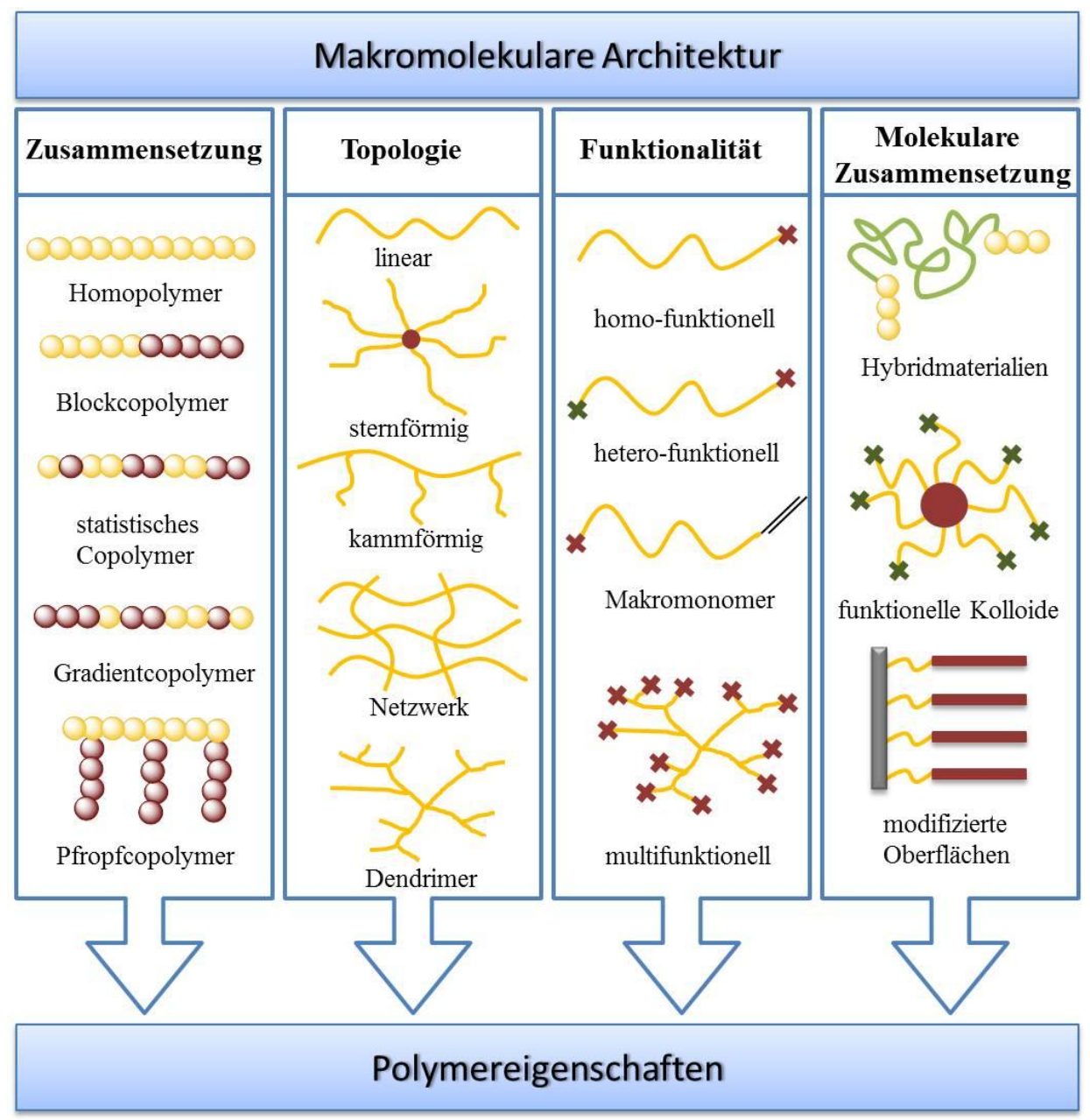

Abbildung 1-1 Mögliche Architekturen von Polymeren.

Durch die Nachteile der radikalischen Polymerisation wie z. B. ihre Unzulänglichkeit bestimmte Polymerstrukturen herzustellen, rücken kontrollierte Polymerisationstechniken wie die (an)ionische oder die reversibeldesaktivierenden radikalischen Polymerisationsmethoden (siehe Abschnitt 2.3) in den Vordergrund. Szwarc führte im Jahr 1956 als erster den Begriff der lebenden anionischen Polymerisation ein, wobei diese Polymerisationsmethode hohe Ansprüche an die Reaktionsbedingungen stellt. ${ }^{10,11}$ Im Gegensatz dazu wurden seit Mitte der 1980er Jahre eine Reihe neuer Polymerisationsverfahren wie die oben genannten reversibel-desaktivierenden radikalischen Polymerisationsmethoden (häufig auch kontrollierte Polymerisation) entwickelt, die sowohl die Vorteile der radikalischen Polymerisation als auch die Vorteile der kontrollierten Polymerisation nutzen. Eine dieser Methoden ist der Reversible Additions- 
Fragmentierungs-Kettentransfer (RAFT)-Prozess. ${ }^{12}$ Mit dieser Technik lassen sich wie bei allen kontrollierten Polymerisationsmethoden die Zusammensetzung, die Topologie, die Funktionalität der Polymere (siehe Abbildung 1-1) und bei der Synthese von Blockcopolymeren die Zahl der Blöcke, die Blocklängen und die Blocklängenverteilungen einstellen. ${ }^{13}$ Blockcopolymere haben in den letzten Jahren großes Interesse in der Forschung und Anwendung hervorgerufen, da sie aufgrund ihrer morphologischen Besonderheiten, die das Ergebnis von Mikrophasenseparation sind, einzigartige Eigenschaften zeigen. Dabei kommt es zur Entmischung der Blockkomponenten und geordnete, periodische Strukturen entstehen (siehe Abschnitt 2.5). Ein besonders anregendes Forschungsgebiet ist die Untersuchung der selbstanordnenden Nanostrukturierung von Blockcopolymeren in dünnen Filmen, wodurch die Blockcopolymere Anwendung in der Nanotechnik z. B. als Vorlage für den Aufbau von nanoskopischen Speichermedien finden. ${ }^{14,15}$ Die Blockcopolymere mit ihrer Vielzahl an unterschiedlichen Architekturen finden zahlreiche technische und medizinische Anwendungen. ${ }^{14}$ Für die Ermöglichung und Optimierung dieser und zahlreicher anderer Entwicklungen ist die Untersuchung der Blockcopolymerfilme aus der Sicht der Grundlagenforschung von großem Interesse. Die Erforschung der durch die eingeschränkte Topologie der Blockcopolymere hervorgerufenen Phänomene trägt zu einem besseren allgemeinen Verständnis der Polymere bei.

Im Rahmen der vorliegenden Arbeit werden phasenseparierte Diblockcopolymere mit eingebauten photo-vernetzbaren Einheiten mittels RAFT-Polymerisation hergestellt. Im Laufe der folgenden Kapitel werden die Blockcopolymere durch die Untersuchung der Topographie und der mechanischen Eigenschaften charakterisiert. Außerdem wird gezeigt, wie die Vernetzung die Selbstorganisation in Blockcopolymeren und deren mechanische Eigenschaften beeinflusst. 


\section{Theorie}

\subsection{Radikalische Polymerisation}

Die radikalische Polymerisation ist aufgrund ihrer Vielseitigkeit eines der wichtigsten Verfahren zur Herstellung von Polymerwerkstoffen. Nahezu 50 \% der großtechnisch produzierten Polymere werden auf diesem Wege hergestellt. ${ }^{2}$ Der große Vorteil dieser Reaktion liegt in ihrer Unempfindlichkeit gegenüber Verunreinigungen. Die radikalische Polymerisation unterteilt sich in die vier Hauptreaktionsschritte Initiierung und Startreaktion, Propagation, Kettentransfer und Terminierung (Schema 2-1). Hierbei steht der Geschwindigkeitskoeffizient $k_{\mathrm{d}}$ für die Zerfallsreaktion, $k_{\mathrm{p}}$ für die Propagation, $k_{\mathrm{tr}}$ für die Transferreaktion und $k_{\mathrm{t}}$ für die Terminierung.

1) Initiierung und Startreaktion

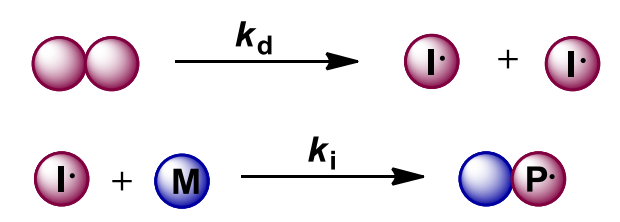

2) Propagation

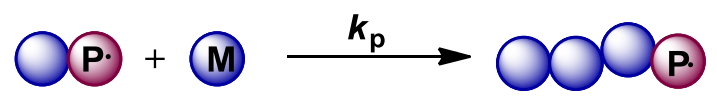

3) Kettentransfer

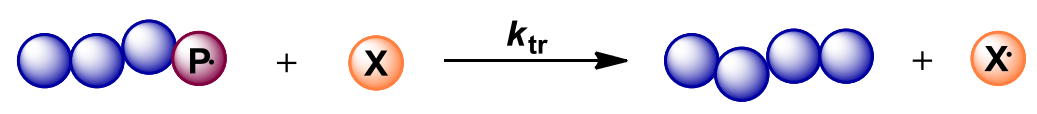

4) Terminierung

Rekombination

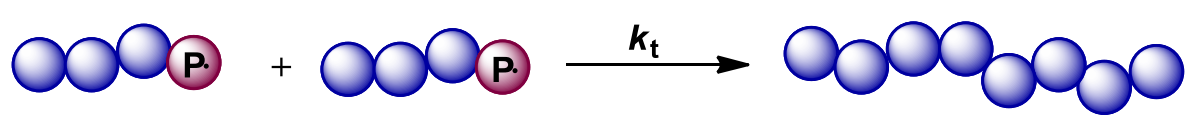

Disproportionierung

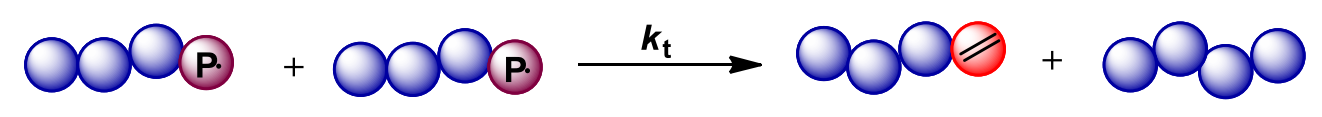

Schema 2-1 Reaktionsschema der radikalischen Polymerisation. 
Bei der Initiierung oder dem Kettenstart wird zuerst das Initiatormolekül thermisch, elektrochemisch oder photochemisch in zwei Initiatorradikale homolytisch gespalten. Danach kann das Initiatorradikal an die KohlenstoffDoppelbindung des Monomers addieren, wobei die Radikalfunktion erhalten bleibt.

Bei der Propagation oder dem Kettenwachstum lagern sich Monomereinheiten an das Initiatorradikal an, wobei Oligomer- und Polymerketten entstehen. Bei der Terminierung oder dem Kettenabbruch kommt es zur Verringerung der Gesamtzahl der Radikale, da sie Rekombinations- und Disproportionierungsreaktionen eingehen können. Bei der Rekombination bilden beide Kettenradikale untereinander eine $\sigma$-Bindung aus und eine Polymerkette entsteht. Bei der Disproportionierung wird ein Wasserstoffatom von einem Kettenradikal auf das zweite übertragen, sodass sich zwei Polymerketten bilden. Dabei verfügt eine Polymerkette über eine polymerisierbare Doppelbindung und die zweite Polymerkette ist, wie bei der Rekombination, ein sog. totes Polymer. Der Kettenabbruch ist ein diffusionskontrollierter Schritt, bei dem sich die Radikale in räumlicher Nähe zueinander befinden müssen.

Neben dem Kettenwachstum und -abbruch kann es auch zu einem Kettentransfer kommen, bei dem die Radikalfunktion auf ein Molekül X (siehe Schema 2-1) übertragen werden kann. Dabei kann das Molekül X ein Monomer, Polymer, Lösungsmittel oder ein Kettentransferagens (engl.: chain transfer agent, CTA) darstellen. Im Gegensatz zur Kettenabbruchsreaktion wird die Reaktion jedoch nicht komplett beendet. Die Übertragung auf die Polymerketten findet nicht nur an den Kettenenden statt. Das Polymer kann auch entlang der wachsenden Kette angegriffen werden, wodurch es zur Bildung von Verzweigungen kommt. Diese Übertragungsreaktion kann intra- (sog. backbiting) und intermolekular erfolgen. ${ }^{16}$ Die Übertragungsreaktion hat keinen Einfluss auf die Wachstums- oder Abbruchgeschwindigkeit. Der größte Vorteil bei der Nutzung eines Übertragungsreagenz liegt in der Kontrolle der Molmassen und somit der Kontrolle über Materialeigenschaften. ${ }^{17}$

Zur Steigerung der Kontrolle über die Molmasse, Dispersität, Endgruppenfunktionalisierung und Polymerarchitektur kam es in den späten 1990er Jahren zu einem großen Forschungsinteresse im Bereich der reversibel-desaktivierenden radikalischen Polymerisationstechniken (RDRP), auf die in Abschnitt 2.3 eingegangen wird. 


\subsection{Copolymerisation}

Copolymerisation nennt man die gemeinsame Polymerisation von zwei oder mehr chemisch verschiedenen Monomeren. Der Mechanismus der Copolymerisation ist dem der Homopolymerisation sehr ähnlich, aber durch die unterschiedlichen Reaktivitäten der Monomere untereinander können verschiedene Typen von Copolymeren entstehen (siehe Abbildung 2-1). Das statistische Copolymer besteht aus einer zufälligen Verteilung der beiden Monomere A und B im Copolymer, wohingegen sich das alternierende Copolymer aus einer regelmäßigen Anordnung der Monomere entlang der Kette zusammensetzt. Das Gradientcopolymer ähnelt dem statistischen Copolymer, wobei sich statistische Copolymere -A-A-B-A-B-B-B-A-

alternierende Copolymere

-A-B-A-B-A-B-A-B-

Gradientcopolymere

-A-A-A-B-A-B-A-A-B-B-B-

\section{Blockcopolymere}

-A-A-A-A-A-B-B-B-B-

Pfropfcopolymere

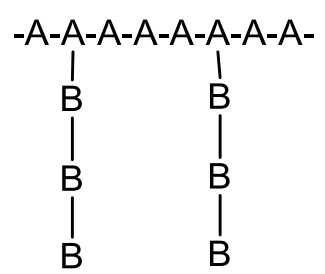

Abbildung 2-1 Verschiedene Typen von Copolymeren bestehend aus dem Monomer A und B. der Anteil eines Monomers im Verlauf der Kette verändert. Blockcopolymere bestehen aus längeren Sequenzen eines Monomers in der Kette (siehe Abschnitt 2.5). Bei den Pfropfcopolymeren werden Blöcke eines Monomers B auf das Rückgrat eines Monomers A aufgepfropft.

Ein Vorteil der Copolymerisation besteht in der Einbringung der Polymereigenschaften der beteiligten Monomere in das Copolymer. Dabei kann bereits ein geringer eingebauter Anteil ausreichen, um eine gewünschte Eigenschaft zu erreichen, wie beispielsweise beim Einbau von Isopren in Polyisobutylen zur Ermöglichung der Vulkanisation zum Butylkautschuk. ${ }^{18}$ In der vorliegenden Arbeit wird ein geringer Monomeranteil von Coumarin in das Copolymer eingebaut, um eine Vernetzung des Polymers zu ermöglichen.

\subsection{Reversibel-Desaktivierende Radikalische Polymerisation}

In der Literatur existieren unterschiedliche Bezeichnungen für Polymerisationstechniken, die ein einheitliches Polymer liefern. Die IUPAC definierte im Jahr 2009 den Begriff reversibel-desaktivierende radikalische 
Polymerisation (engl.: reversible-deactivation radical polymerization, RDRP) zum Oberbegriff dieser Techniken, die vorher bekannt waren als z. B. lebende oder kontrollierte Polymerisationen. ${ }^{19}$ Szwarc führte im Jahr 1956 als erster den Begriff lebende Polymerisation für anionische Polymerisationen ein.10,11,20 Der große Vorteil bei der (an)ionischen Polymerisation ist die Darstellung einheitlicher Polymere mit vorbestimmten Molmassen und niedrigen Dispersitäten ohne das Auftreten irreversibler Abbruchreaktionen. Des Weiteren finden keine Übertragungsreaktionen statt (Voraussetzung hierfür ist das Fehlen von Verunreinigungen).

Die wichtigsten Vertreter der RDRP sind vor allem die Nitroxid-vermittelte Polymerisation (NMP), ${ }^{21-24}$ die Atom-Transfer radikalische Polymerisation (ATRP) $9,25,26$ und die Reversible Additions-Fragmentierungs-Kettentransfer (RAFT)- Polymerisation27,28. Der RDRP-Technik liegt das Prinzip zugrunde, die propagierenden Ketten durch Zugabe eines Reagenz reversibel zu desaktivieren. Die Desaktivierung kann dabei durch eine reversible Terminierung (NMP und ATRP) oder durch einen reversiblen Kettentransfer (RAFT) erreicht werden. Das zugegebene Reagenz lagert sich reversible an die Radikalspezies an, wodurch eine desaktivierte Spezies entsteht, die keine weiteren Monomere addieren kann. Somit liegt ein dynamisches Gleichgewicht zwischen einer aktiven (freie Radikale) und einer desaktivierten Spezies vor (Schema 2-2).

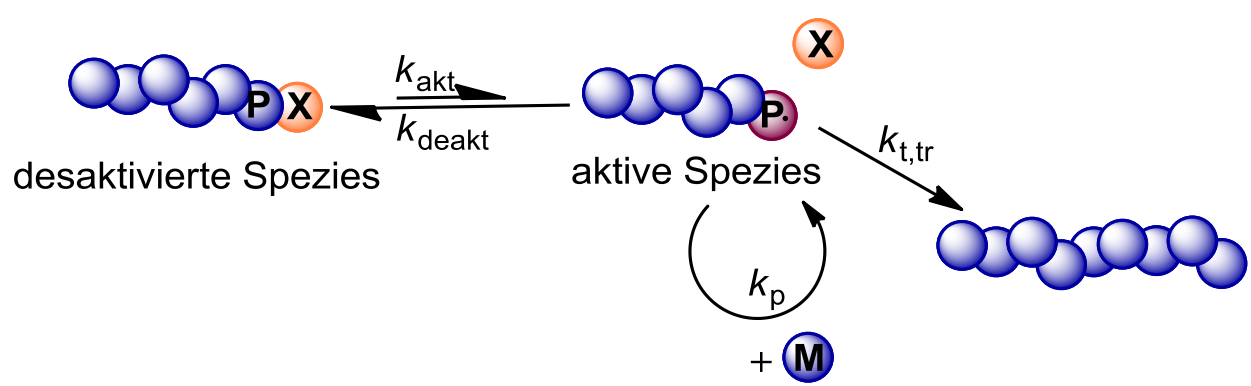

Schema 2-2 Dynamisches Gleichgewicht des RDRP-Prinzips.

Bei der ATRP und der NMP liegt das dynamische Gleichgewicht immer auf der Seite der desaktivierten Spezies, wodurch die Anzahl der propagationsfähigen Radikale in der Polymerisation stark reduziert wird. Abbruch- und Übertragungsreaktionen werden hierbei nicht vollständig unterdrückt, aber durch die niedrige Radikalkonzentration finden diese Reaktionen nur selten statt. Der abweichende Mechanismus der RAFT-Polymerisation wird in Abschnitt 2.3.1 näher erläutert. 
Die RDRP verbindet die Vorteile einer konventionellen radikalischen Polymerisation (wie z. B. schnelle und einfache Reaktionsführung) mit den Vorteilen einer kontrollierten Polymerisation (wie z. B. Einfluss auf Dispersität). Bei den RDRP-Techniken bleibt das aktivierbare Zentrum an den Ketten nach Abbruch der Polymerisation oder nach vollständigem Verbrauch des Monomers erhalten. Somit können bestimmte funktionelle Gruppen in die Polymere eingebaut werden oder es können weitere Polymerisationen an den Ketten durchgeführt werden. Insgesamt ermöglicht die RDRP die Herstellung von Polymeren mit engen Molmassenverteilungen und eine Vielzahl komplexer Polymerstrukturen wie z. B. Blockcopolymere. ${ }^{29,30}$

Da im Rahmen der vorliegenden Arbeit ausschließlich mit der RAFTPolymerisation gearbeitet wurde, wird diese Polymerisationstechnik sehr ausführlich im folgenden Abschnitt besprochen.

\subsubsection{Reversibler Additions-Fragmentierungs-Kettentransfer (RAFT)}

Der Reversible Additions-Fragmentierungs-Kettentransfer (RAFT)-Prozess wurde im Jahr 1998 von der Commonwealth Scientific and Industrial Research Organisation (CSIRO) vorgestellt.12 Moad et al. haben seitdem in vier ausführlichen Reviews die Entwicklungen und Vorzüge der RAFT-Polymerisation durch die zahlreichen, unterschiedlich forschenden Arbeitsgruppen zusammengefasst. ${ }^{28,31-33}$ Die RAFT-Polymerisation ist in Bezug auf einsetzbare Monomere und Reaktionsbedingungen eine der vielseitigsten Techniken der RDRP.31,32,34-36 Durch die sehr hohe Toleranz gegenüber funktionellen Gruppen erlaubt die RAFTPolymerisation die Herstellung hochfunktionalisierter Materialien. ${ }^{37}$ Weiterhin können Polymere mit komplexen Strukturen wie zum Beispiel Block-, Stern- und Pfropfpolymere erzeugt werden.27,29,38-40 Die RAFT-Polymerisation ist in Dispersions- oder Emulsionspolymerisation, in Substanz, in superkritischen Lösungsmitteln oder auch in wässrigen Lösungen durchführbar.41-45

Die Kontrollagenzien in der RAFT-Polymerisation sind in der Regel Reagenzien aus der Gruppe der Thiocarbonylthio-Verbindungen. Die allgemeine Struktur wird in der Abbildung 2-2 gezeigt. Dabei kennzeichnet R die Abgangsgruppe und $\mathrm{Z}$ die stabilisierende Gruppe. ${ }^{46,47}$ 
<smiles>[R]SC([Z])=S</smiles>

Abbildung 2-2 Allgemeine Strukturformel des RAFT-Agens.

Der Ablauf der RAFT-Polymerisation entspricht in den grundlegenden Reaktionsschritten dem einer konventionellen radikalischen Polymerisation (Initiierung, Wachstum, Terminierung). Allerdings wird dem Polymerisationssystem noch das oben beschriebene RAFT-Agens hinzugegeben. In Schema 2-3 sind die elementaren Reaktionsschritte des allgemein akzeptierten Mechanismus der RAFT-Polymerisation dargestellt.

1) Initiierung und Startreaktion

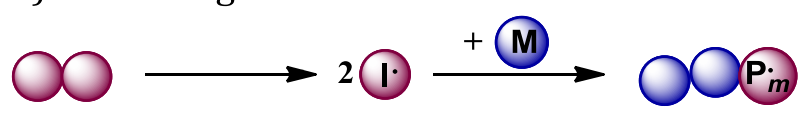

2) Vorgleichgewicht

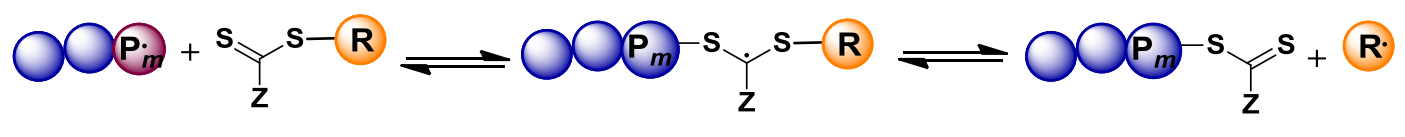

3) Propagation

Intermediat

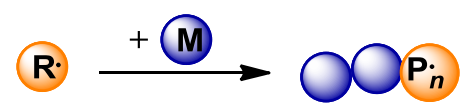

4) Hauptgleichgewicht

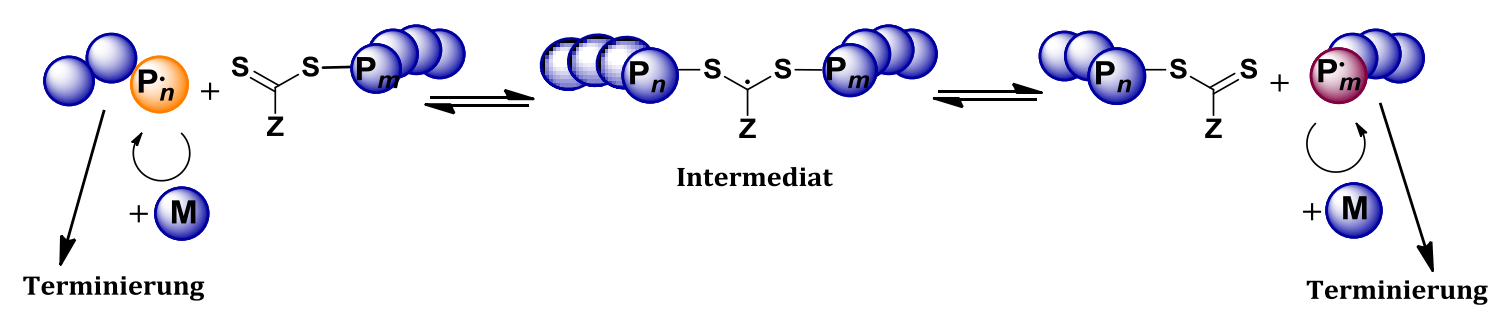

Schema 2-3 Der allgemeine Mechanismus der RAFT-Polymerisation.

Am Anfang der Polymerisation entstehen durch den Zerfall eines Initiatormoleküls Radikale, die durch Addition an Monomermoleküle wachsende Polymerketten starten (Initiierung/Startreaktion). Diese Makroradikale addieren schnell an die reaktive Kohlenstoff-Schwefel-Bindung der Thiocarbonylthio-Funktion des RAFTAgens (Vorgleichgewicht) und es entsteht ein intermediäres RAFT-Radikal. Die ZGruppe des RAFT-Agens soll zum einen die Reaktivität der Kohlenstoff-SchwefelBindung für den Radikalangriff erhöhen und zum anderen das entstandene RAFT- 
Radikal stabilisieren. Bei einer zu starken Stabilisierung kann es sein, dass es zu einer Retardierung der Reaktion kommt, da die Fragmentierung der R-Gruppe verzögert passiert.

Das RAFT-Intermediat kann nun die Rückreaktion der Addition eingehen oder es kann die Abgangsgruppe $\mathrm{R}$ abgespalten werden und ein makroRAFT-Agens (makromolekulares RAFT-Agens) bleibt zurück. Die R-Gruppe kann dann durch Reaktion mit weiteren Monomermolekülen die Polymerisation reinitiieren und eine neue propagierende Kette starten, die wiederum an ein RAFT-Agens addiert. Um diese Reaktionsbedingungen zu erreichen, muss die R-Gruppe sowohl eine gute Abgangsgruppe im Vergleich zur Polymerkette sein, als auch das eingesetzte Monomer gut reinitiieren können. Die Fragmentierung der R-Gruppe läuft umso schneller ab, desto stabiler das durch die Fragmentierung gebildete Radikal ist. Darum eignen sich als R-Gruppe besonders elektrophile oder über große Substituenten verfügende Verbindungen. ${ }^{48}$ Damit die R-Gruppe schnell an das Monomer addiert und eine gute Initiierung neuer Ketten gegeben ist, sollte die RGruppe passend zum verwendeten Monomer gewählt werden. Ansonsten steigt die Wahrscheinlichkeit für Abbruchreaktionen.

Zusammenfassend lässt sich sagen, dass die Effektivität des RAFT-Agens von der Übertragungskonstante abhängig ist, die wiederum durch die Wahl der R- und ZGruppe bestimmt wird sowie der Wahl des Monomers. ${ }^{12,38}$ Weiterhin ist die Substanzklassenwahl des RAFT-Agens entscheidend, wobei man zwischen Dithioester, Xanthaten (auch als MADIX- Macromolecular Design via the Interchange of Xanthate bezeichnet), Dithiocarbamaten und Trithiocarbonaten unterscheidet.28,32,49-52 Zur Planung einer RAFT-Polymerisation ist die Wahl des passenden RAFT-Agens von entscheidender Bedeutung. Eine Übersicht über geeignete RAFT-Agenzien für unterschiedliche Monomere veröffentlichten Moad et. al (Schema 2-4).27,48 


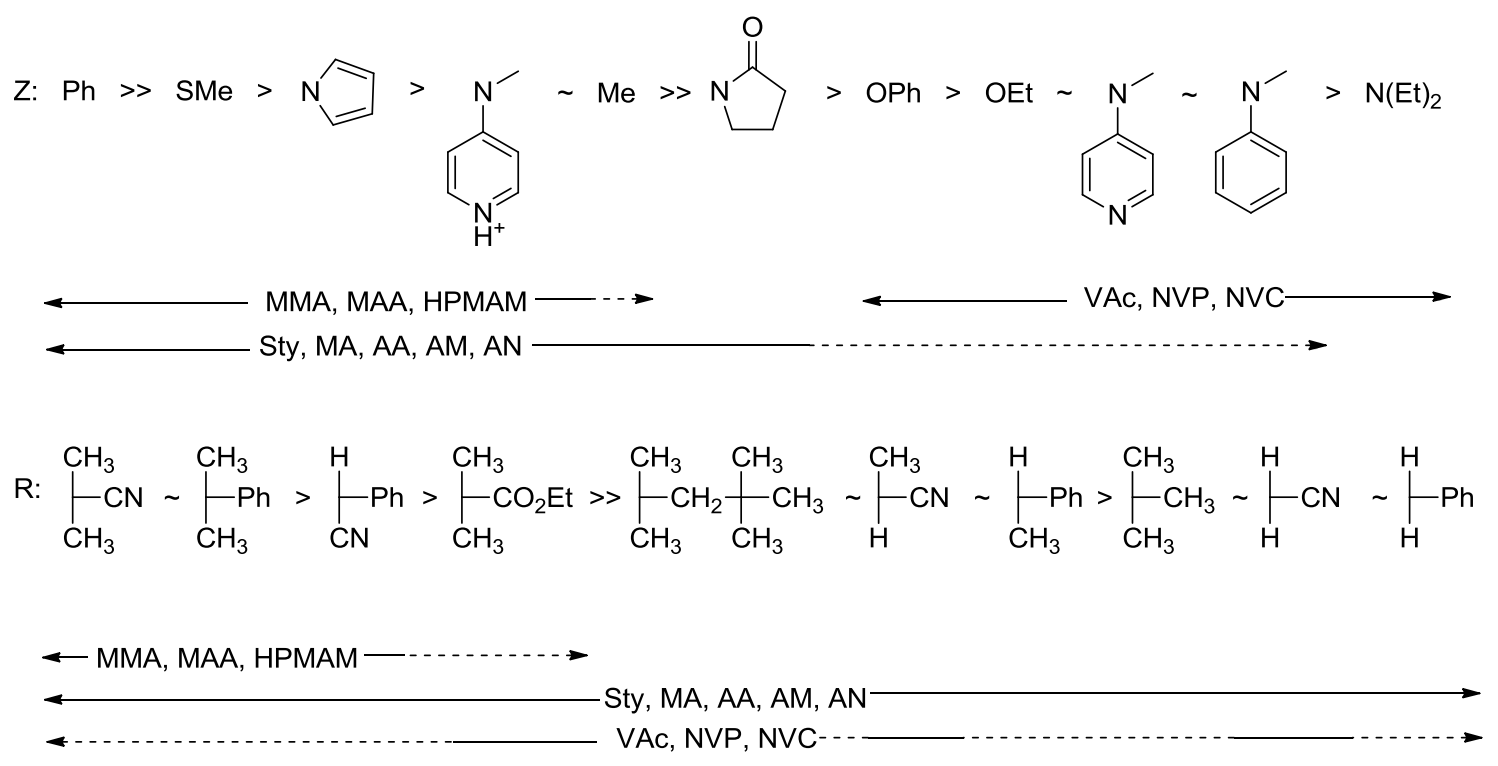

Schema 2-4 Überblick passender RAFT-Gruppen für verschiedene Monomere. ${ }^{27}$

Sobald das ursprüngliche RAFT-Agens durch die Reinitiierung der R-Gruppen aufgebraucht ist, ist das Vorgleichgewicht überwunden. In dem Hauptgleichgewicht reagiert das makroRAFT-Agens. Hierbei findet ein schneller Austausch von wachsenden Ketten und solchen, die an ein RAFT-Agens gebunden sind, statt. Dieser Mechanismus führt zu einer Angleichung der Wachstumswahrscheinlichkeit aller Ketten, wodurch eine enge Molmassenverteilung entsteht. ${ }^{27}$ Daraus ergibt sich, dass der Anstieg des Zahlenmittels der Molmasse direkt proportional zum Umsatz ist.

Am Ende der Polymerisation besitzt eine große Mehrheit der Makromoleküle die Thiocarbonylthio-Gruppe am einen und die R-Gruppe am anderen Ende der Kette. Die Polymerketten mit den RAFT-Endgruppen werden als lebende Ketten bezeichnet, wohingegen die Ketten, die durch Terminierung entstehen, tote Ketten genannt werden. Das Verhältnis von lebenden zu toten Polymerketten kann durch das Verhältnis der Konzentration des RAFT-Agens zur Konzentration der durch den Initiatorzerfall initiierenden Radikale gesteuert werden. ${ }^{31}$ Die lebenden Polymerketten können als makroRAFT-Agenzien in weiteren Polymerisationen reaktiviert werden, wodurch unter anderem die Herstellung von Blockcopolymeren ermöglicht wird. ${ }^{27}$

Die Kinetik der RAFT-Polymerisation gleicht im Idealfall der einer konventionellen radikalischen Polymerisation und die Konzentration der propagierenden Radikale wird nicht verändert. Somit laufen Kettenwachstum und Kettenabbruch in der 
RAFT-Polymerisation mit der gleichen Geschwindigkeit wie in der konventionellen radikalischen Polymerisation ab. Für das ideale Verhalten der RAFTPolymerisation wäre es erforderlich, dass die R-Gruppe dieselbe Reaktivität wie ein propagierendes Makroradikal aufweist. Da dies nur in Näherung zutrifft, müssen einige Betrachtungen bezüglich des RAFT-Vorgleichgewichts und dem Einfluss der R-Gruppe gemacht werden. Die Initialisierung beschreibt den Verbrauch des RAFT-Agens mit der ursprünglichen Abgangsgruppe. ${ }^{53}$ Während der Initialisierung wird die ursprüngliche Abgangsgruppe R durch propagierende Radikale ersetzt. Wenn die Abgangsgruppe R schlechter stabilisiert wird als das propagierende Radikal, besteht eine größere Wahrscheinlichkeit, dass das RAFTIntermediat zurück in das ursprüngliche RAFT-Agens und das propagierende Radikal zerfällt. Daraus folgt, dass während der Initialisierung nicht alle Ketten gleichmäßig wachsen und bei niedrigem Umsatz das Zahlenmittel der Molmasse der Polymerketten über dem aufgrund der RAFT-Polymerisation erwartetem Wert liegt. Dieses Verhalten wird als Hybridverhalten bezeichnet.

Wenn der Geschwindigkeitskoeffizient für die Propagation der Abgangsgruppe groß ist, dann kann ein schneller Übergang zum Hauptgleichgewicht stattfinden..$^{53}$ Es muss darauf geachtet werden, dass das aus der Abgangsgruppe entstehende Radikal genügend reaktiv für eine Reinitiierung ist. Ist dies nicht der Fall, kann es aufgrund von Terminierungsreaktionen zu einer Verlangsamung der Reaktionsgeschwindigkeit und zu einer langen Induktion in der Anfangsphase der Polymerisation kommen. ${ }^{54}$

Neben der Möglichkeit einer RAFT-bedingten Induktion gibt es einen weiteren beobachteten Effekt, der eine Verzögerung bei der Polymerisation aufweisen kann. Dieser Effekt wird als Retardierung bezeichnet. Dabei nimmt die Polymerisationsgeschwindigkeit mit zunehmender RAFT-Agens-Konzentration ab. ${ }^{55}$ Schema 2-5 zeigt zwei unterschiedliche Mechanismen, die zur Retardierung führen können. 


$$
\begin{aligned}
& \text { 3-Arm-Stern } \quad \mathrm{P}_{\mathrm{n}}-\mathrm{S}_{\mathrm{P}_{0}}^{\mathrm{Z}} \mathrm{S}_{-\mathrm{P}_{\mathrm{m}}} \quad \begin{array}{l}
\text { mögliches irreversibles } \\
\text { Terminierungsprodukt }
\end{array} \\
& \uparrow+P_{\circ}^{\cdot}
\end{aligned}
$$

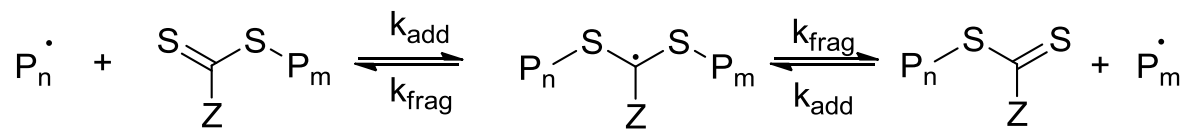

$$
\begin{aligned}
& \mathrm{k}_{\text {reak }} \|_{\mathrm{t} \text {,int-int }}
\end{aligned}
$$

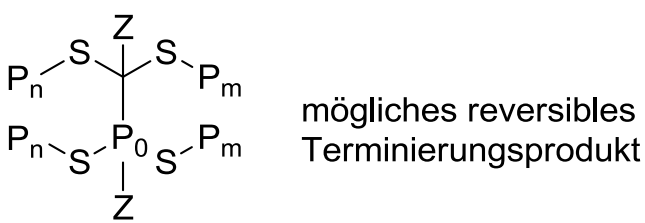

Schema 2-5 Darstellung der Reaktionen, die zum Effekt der Retardierung führen können. ${ }^{56}$

Der erste Fall beschreibt die reversible Terminierungsreaktion zweier RAFTIntermediate. ${ }^{57-59}$ Im zweiten Fall kann das RAFT-Intermediat in einer irreversiblen Terminierungsreaktion mit einem weiteren Polymerradikal reagieren, wobei ein 3-Arm-Stern gebildet wird.59-62 Durch die irreversible Terminierung wird die Radikalkonzentration kleiner, was wiederum die Retardierung verursacht. Bei der RAFT-Polymerisation konnten aber bis heute keine 3-Arm-Sterne nachgewiesen werden, wodurch es zur Annahme eines dritten Falls kam. Dabei reagiert der entstandene 3-Arm-Stern mit einem propagierenden Radikal, es entsteht wieder das RAFT-Intermediat und eine tote Kette. ${ }^{63,64}$

\subsection{Molmassenverteilungen}

Synthetische Polymere besitzen im Gegensatz zu biologischen Systemen keine einheitlichen Molmassen. Zur Beschreibung der Molmassenverteilung werden verschiedene charakteristische Kenngrößen verwendet. Eine wichtige Kenngröße ist das Zahlenmittel der Molmasse $\bar{M}_{\mathrm{n}}$, das aus dem Stoffmengenanteil der Moleküle $x_{i}$ der Kettenlänge $i$ und der Molmasse der Moleküle $M_{i}$ mit der Kettenlänge $i$ berechnet wird. Die Berechnung von $\bar{M}_{\mathrm{n}}$ wird in Gleichung (1) dargestellt. 


$$
\bar{M}_{\mathrm{n}}=\sum_{i=1}^{\infty} x_{i} M_{i}=\frac{\sum_{i=1}^{\infty} N_{i} M_{i}}{\sum_{i=1}^{\infty} N_{i}}
$$

Dabei kann der Stoffmengenanteil auch über die Zahl der Moleküle $N_{\mathrm{i}}$ der Kettenlänge $i$ ausgedrückt werden. Das Gewichtsmittel der Molmasse $\bar{M}_{\mathrm{w}}$ stellt eine weitere wichtige Kenngröße dar. Die Berechnung erfolgt aus dem Gewichtsmittel der Moleküle $w_{\mathrm{i}}$ mit der Kettenlänge $i$ und der Molmasse $M_{\mathrm{i}}$ mit der Kettenlänge $i$ und wird in Gleichung (2) dargestellt.

$$
\bar{M}_{\mathrm{w}}=\sum_{i=1}^{\infty} w_{i} M_{i}=\frac{\sum_{i=1}^{\infty} N_{i} M_{i}^{2}}{\sum_{i=1}^{\infty} N_{i} M_{i}}
$$

Je ähnlicher die Molmassen der einzelnen Polymerketten sind, desto näher liegen die Werte für $\bar{M}_{\mathrm{n}}$ und $\bar{M}_{\mathrm{w}}$ beieinander. Der Quotient aus Gewichts- und Zahlenmittel einer Molmassenverteilung ist ein Maß für die Breite der Molmassenverteilung und wird mit der Kenngröße Dispersität $Ð$ gekennzeichnet (siehe Gleichung (3))

$$
Ð=\frac{\bar{M}_{\mathrm{w}}}{\bar{M}_{\mathrm{n}}}
$$

Biologische Makromoleküle, die eine einheitliche Kettenlänge besitzen, haben einen Dispersitätswert von $Đ=1$. Je größer der Dispersitätswert wird, desto breiter wird die Molmassenverteilung.

Die Dispersität kann auch mit dem mittleren Polymerisationsgrad $\bar{P}_{\mathrm{n}}$ ausgedrückt werden, wenn sich die Molmassenverteilung einer Poisson-Verteilung nähert, wie z. B. bei der RDRP.

$$
\bigoplus=1+\frac{1}{\bar{P}_{\mathrm{n}}}
$$

Der Polymerisationsgrad beschreibt die Kettenlänge in Monomereinheiten (siehe Gleichung (5)).

$$
\overline{P_{\mathrm{n}}}=\frac{X \cdot[\mathrm{M}]_{0}}{[\mathrm{I}]_{0}}
$$


Dabei stellt $X$ den Monomerumsatz, $[\mathrm{I}]_{0}$ und $[\mathrm{M}]_{0}$ die Anfangskonzentration des Initiators und des Monomers dar.

Die Terminierungsart hat einen großen Einfluss auf den resultierenden Dispersitätswert. Bei einer radikalischen Polymerisation unter stationären Bedingungen wird im Fall einer Terminierung der Radikale über Kombination eine Dispersität von 1,5 und bei einer Terminierung über Disproportionierung eine Dispersität von 2 erwartet. ${ }^{65,66}$ Die Monomer- und Initiatorkonzentration sowie die Temperatur sind aber bei einer radikalischen Polymerisation nur näherungsweise konstant, wodurch ein Dispersitätswert von $Ð>1,5$ erwartet wird. Der Vorteil der RDRP liegt in der guten Molmassenkontrolle, wodurch eine niedrige Dispersität $(\bigoplus<1,5)$ erwartet wird. Die Polydispersität ist somit ein Gütekriterium für die Polymerisationskontrolle.65 Bei einer typischen RAFT-Polymerisation werden Dispersitäten zwischen 1,1 und 1,2 erwartet. $^{12}$

Für die ideale, kontrollierte RAFT-Polymerisation ist eine Berechnung der theoretischen Molmasse nach Gleichung (6) möglich: ${ }^{31}$

$$
\bar{M}_{\mathrm{n}}^{\text {theo }}=\frac{X \cdot[\mathrm{M}]_{0} \cdot M_{\text {Monomer }}}{[\mathrm{RAFT}]_{0}}+M_{\mathrm{RAFT}} .
$$

Die Anzahl der Polymerketten entspricht hierbei der Anzahl der lebenden Zentren und wird durch die Konzentration des Kontrollagens wiedergegeben. Weiterhin ist festzuhalten, dass der Anstieg des Zahlenmittels der Molmasse direkt proportional zum Umsatz $X$ ist. Da jede Kette eine RAFT-Einheit trägt, muss die Molmasse des RAFT-Agens $M_{\text {RAFT }}$ berücksichtigt werden.

Durch Terminierung kann es bei der RAFT-Polymerisation zur Bildung von toten Polymerketten kommen. Die Anzahl der toten Ketten ist abhängig von der Anfangskonzentration des Initiators $[\mathrm{I}]_{0}$, der Initiatoreffizienz $f$, dem Geschwindigkeitskoeffizienten des Initiatorzerfalls $k_{\mathrm{d}}$, der Reaktionszeit $t$ und der Anzahl der bei der Terminierung gebildeten Polymerketten $d$. Die Terminierung kann bei der Berechnung der theoretischen Molmasse durch Einbau der Parameter in Gleichung (6) berücksichtigt werden und es folgt:

$$
\bar{M}_{\mathrm{n}}^{\text {theo }}=\frac{X \cdot[\mathrm{M}]_{0} \cdot M_{\text {Monomer }}}{[\mathrm{RAFT}]_{0}+[\mathrm{I}]_{0} \cdot d \cdot f \cdot\left(1-\mathrm{e}^{-k_{\mathrm{d}} \cdot t}\right)}+M_{\mathrm{RAFT}}
$$


Bei vielen RAFT-Polymerisationen ist die Zahl der initiierenden Radikale im Verhältnis zu der RAFT-Konzentration vernachlässigbar klein, weshalb das tote Polymer bei der Berechnung der theoretischen Molmasse in dieser Arbeit nicht berücksichtigt wurde. Die Berechnung der theoretischen Molmasse erfolgte in dieser Arbeit nach Gleichung (22).

Bei der Copolymerisation von zwei Monomeren A und B wurde für die Berechnung der theoretischen Molmasse Gleichung (8) verwendet:

$$
\bar{M}_{\mathrm{n}}^{\text {theo }}=\frac{X \cdot\left[\left(\left[\mathrm{M}_{\mathrm{A}}\right]_{0} \cdot M_{\mathrm{A}}\right)+\left(\left[\mathrm{M}_{\mathrm{B}}\right]_{0} \cdot M_{\mathrm{B}}\right)\right]}{[\mathrm{RAFT}]_{0}}+M_{\mathrm{RAFT}}
$$

wobei $M_{\mathrm{A}}$ und $M_{\mathrm{B}}$ die Molmassen der Monomere A und B darstellen und $X$ den Gesamtumsatz.

Die Gelpermeationschromatographie (GPC) ist eine wichtige Methode zur Bestimmung von Molmassenverteilungen. Die stationäre Phase besteht aus einem porösen, gequollenen Polymernetzwerk mit breiter Porengrößenverteilung. Das gelöste Polymer durchläuft diese Säule mit einer konstanten Flussrate bei einer konstanten Temperatur. Die Fraktionierung erfolgt durch das unterschiedliche hydrodynamische Volumen $V_{\mathrm{h}}$ der Polymermoleküle. Das Volumen wird aus der intrinsischen Viskosität $[\eta]$ und der Molmasse $M$ berechnet und durch Gleichung (9) beschrieben:

$$
V_{\mathrm{h}}=[\eta] \cdot M
$$

Je größer das hydrodynamische Volumen eines Moleküls ist, desto geringer ist die Retentionszeit in der Säule. Die Polymerketten können nach der Separation unterschiedlich detektiert werden, z. B. durch die Veränderung des Brechungsindex (RI) oder UV-Absorption. Die Molmassensverteilung wird in einem GPChromatogramm in logarithmierter Form aufgetragen. ${ }^{67}$

Da die GPC eine Relativmethode ist, muss das System mit einem passenden Standard kalibriert werden. Sollte keine passende Kalibrierungskurve für das zu untersuchende Polymer zur Verfügung stehen, kann das System universell kalibriert werden.68 Dabei wird das zu untersuchende Polymer mit den Mark-Houwink-Parametern $K$ und $a$ eines bekannten Polymers kalibriert. 


$$
[\eta]=K \cdot M^{a}
$$

Die Mark-Houwink-Parameter müssen für jedes Polymer-Lösungsmittel-Paar empirisch bestimmt werden, wobei $K$ von dem verwendeten Monomer anhängig ist und $a$ beschreibt die hydrodynamische Wechselwirkung zwischen dem Polymer und dem Lösungsmittel. Die Mark-Houwink-Parameter sind für eine große Anzahl an Polymeren bekannt und können somit für eine universelle Kalibrierung benutzt werden. Durch die vorangegangenen Annahmen kann folgende Gleichung abgeleitet werden: 69

$$
\log M_{2}=\frac{1}{1+a_{2}} \log \frac{K_{1}}{K_{2}}+\frac{1+a_{1}}{1+a_{2}} \log M_{1}
$$

wobei der Index $1 \mathrm{zu}$ dem bekannten Polymer für die Kalibration gehört und der Index $2 \mathrm{zu}$ dem zu untersuchenden Polymer. Grundvoraussetzung für die Bestimmung der Molmassen der Polymere sind bekannte Mark-HouwinkParameter. Wenn dies nicht der Fall ist, kann die Molmasse der Polymere beispielsweise auch durch Viskositätsmessungen oder Lichtstreudetektoren bestimmt werden. ${ }^{70,71}$

\subsection{Blockcopolymere}

Blockcopolymere (BCP) sind Makromoleküle, die aus mindestens zwei Blöcken kovalent miteinander verbundener Homopolymere oder Copolymere aufgebaut sind. Durch diese sequentielle Verknüpfung können Blockcopolymere im Vergleich zu den sie bildenden Homopolymeren ein völlig neues Eigenschaftsprofil erhalten. Entsprechend der Anzahl der Blöcke handelt es sich um Diblock-, Triblock- und Multiblockcopolymere. Es gibt viele unterschiedliche Möglichkeiten die Blöcke anzuordnen. In Abbildung 2-3 werden die möglichen Sequenzmuster durch Anzahl der Monomerbausteine und Topologie (linear und verzweigt) klassifiziert. ${ }^{72,73}$ Jede farbliche Kette repräsentiert einen Block bestehend aus einer linearen Polymerkette eines Monomerbausteins, wobei drei unterschiedliche Monomerbausteine $\mathrm{A}, \mathrm{B}$ und $\mathrm{C}$ dargestellt sind (blau, rot und grün). 


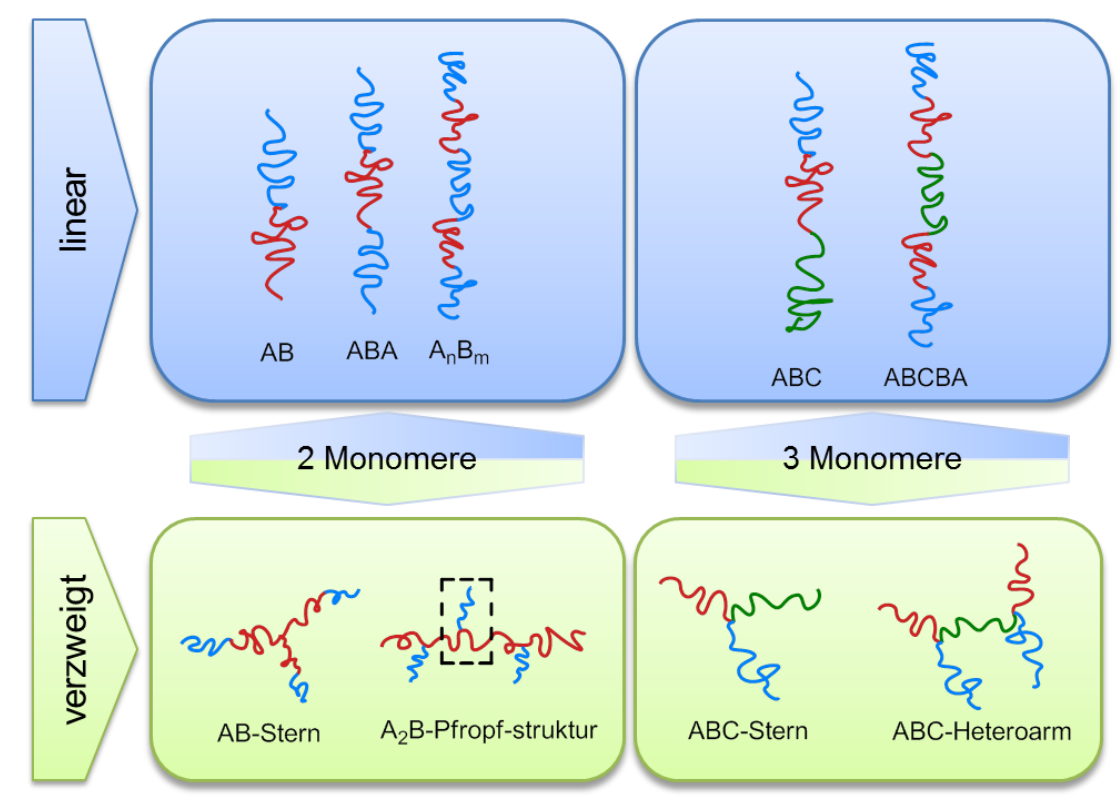

Abbildung 2-3 Blockcopolymerarchitekturen.

Durch definierte Synthesemöglichkeiten können verschiedene Eigenschaftskombinationen, wie plastisch-elastisch (hart-weich) oder hydrophil-hydrophob erzielt werden.29,74-76 Es sind drei Methoden zur Herstellung von Blockcopolymeren bekannt:2,77

- Bei der sequentiellen Monomeraddition fungiert der entstandene Polymerblock A als Makroinitiator für die Polymerisation des zweiten Monomers B usw. Dabei führt der monofunktionelle Polymerisationsstart in zwei Stufen zu AB-Strukturen und in drei Stufen zu ABA- oder ABCStrukturen. Die Herstellung von ABA-Strukturen ist jedoch auch in zwei Stufen durch Einsatz eines bifunktionellen Initiators möglich.

- Die Kopplung von zwei unterschiedlich funktionalisierten Homopolymeren ermöglicht die Bildung von Di-, Tri- bis zu Multiblockcopolymeren sowie Sterncopolymere. Dabei werden die einzelnen Polymerblöcke separat hergestellt, um sie anschließend in einer Kupplungsreaktion miteinander zu verbinden.

- Die site-transformation ist eine Variante der sequentiellen Monomeraddition, wobei diese Variante durch die Reaktivitätsunterschiede der Monomere in mehreren Stufen durchgeführt werden muss. Sie ermöglicht die Kombination verschiedener Mechanismen zur Realisierung von völlig neuen Blockkombinationen. 
Die Synthese von Blockcopolymeren ist ein wichtiger Aspekt der RDRP. Zunächst war die Synthese von Blockcopolymeren nur der anionischen Polymerisation vorbehalten. Diese hat aber den Nachteil, sehr empfindlich gegenüber Verunreinigungen und auch nur für eine begrenzte Anzahl von Monomeren anwendbar zu sein. Durch die Entwicklung neuer Alternativen können inzwischen alle kontrollierten Reaktionsverfahren eingesetzt werden. Sie bieten ebenfalls die Möglichkeit der Synthese von Blockcopolymeren unterschiedlichster Architekturen, besitzen den Vorteil der einfachen Reaktionsführung und weisen eine große Anzahl anwendbarer Monomere auf.

\subsubsection{Mikrophasenseparation in Blockcopolymeren}

In Blockcopolymeren sind die unterschiedlichen Polymersegmente kovalent miteinander verbunden, sodass eine Entmischung der unverträglichen Komponenten nur im Bereich der Längenskalen der Polymerketten stattfinden kann. Somit kommt es zur Mikrophasenseparation (MPS). Die Dimension dieser sich bildenden Mikrodomänen liegt bei ca. 10 bis $100 \mathrm{~nm} .^{76}$

Um die MPS besser verstehen zu können, wird in diesem Abschnitt zuerst die Mischbarkeit von Polymeren erklärt. Die Mischbarkeit zweier Polymere ist abhängig von der Differenz der Gibbs-Energie $\Delta G_{\mathrm{m}}$ zwischen dem phasenseparierten (zweiphasig) und dem homogenen Zustand (einphasig) beider Polymere. Diese Differenz muss für die Mischbarkeit einen negativen Wert annehmen (siehe Gleichung (12)). ${ }^{78}$

$$
\Delta G_{\mathrm{m}}=\Delta H_{\mathrm{m}}-T \Delta S_{\mathrm{m}}
$$

Hierbei ist $\Delta H_{\mathrm{m}}$ die Mischungsenthalpie, $\Delta S_{\mathrm{m}}$ die Mischungsentropie und $T$ die Temperatur. Die Mischungsenthalpie resultiert aus der Wechselwirkung der miteinander in Kontakt tretenden Moleküle, die attraktiv oder repulsiv sein kann. Die Entropie beschreibt das Bestreben der Komponenten, möglichst viele Anordnungen annehmen zu können. Sie begünstigt die Bildung einer Mischphase. Die Mischungsenthalpie und Mischungsentropie können nach der Flory-HugginsTheorie wie folgt beschrieben werden:77,78

$$
\Delta H_{\mathrm{m}}=R T \chi \Phi_{\mathrm{A}} \Phi_{\mathrm{B}}
$$




$$
\Delta S_{\mathrm{m}}=-R\left(\frac{\Phi_{\mathrm{A}}}{N_{\mathrm{A}}} \ln \Phi_{\mathrm{A}}+\frac{\Phi_{\mathrm{B}}}{N_{\mathrm{B}}} \ln \Phi_{\mathrm{B}}\right) .
$$

Dabei sind $\Phi_{\mathrm{A}}$ und $\Phi_{\mathrm{B}}$ der Volumenanteil, $N_{\mathrm{A}}$ und $N_{\mathrm{B}}$ der Polymerisationsgrad der Blöcke A und B, $R$ ist die Gaskonstante und $\chi$ der Entmischungsparameter (oder auch Flory-Huggins-Parameter) der beiden Monomere. Die Mischungsentropie hat mit zunehmender Polymerkettenlänge einen zunehmend geringeren Einfluss, da die Anzahl der möglichen Anordnungen in der Mischung abnimmt. Daraus folgt, dass je größer der Polymerisationsgrad $N$ ist, umso eher tritt eine Entmischung auf. Die Mischungsenthalpie hängt maßgeblich vom Entmischungsparameter $\chi$ ab, der die Wechselwirkungen zwischen dem A- und B-Block beschreibt. ${ }^{79} \chi$ ergibt sich nach Gleichung (15).

$$
\chi=\left(\frac{Z}{k_{\mathrm{B}} T}\right)\left[\varepsilon_{\mathrm{AB}}-\frac{1}{2}\left(\varepsilon_{\mathrm{AA}}+\varepsilon_{\mathrm{BB}}\right)\right]
$$

Hier sind $z$ die Anzahl der direkt benachbarten Monomereinheiten pro Monomer in einer Polymerkette, $k_{\mathrm{B}}$ die Boltzmannkonstante, $T$ die Temperatur, $\varepsilon_{\mathrm{AB}}, \varepsilon_{\mathrm{AA}}$ und $\varepsilon_{\mathrm{BB}}$ die Wechselwirkungsenergien der Monomere $\mathrm{A}-\mathrm{B}, \mathrm{A}-\mathrm{A}$ und $\mathrm{B}-\mathrm{B}$ in den unterschiedlichen Blöcken. Der Entmischungsparameter ist bei repulsiven Wechselwirkungen positiv und bei attraktiven Wechselwirkungen negativ. Je größer der Wert $\chi$ ist, desto ungünstiger ist der Kontakt zwischen den Segmenten der verschiedenen Polymere. Außerdem ist $\chi$ proportional zu $T^{-1}$ und nimmt folglich mit steigender Temperatur ab.77,78

Phasenseparation tritt dann auf, wenn $\Delta G_{\mathrm{m}}$ positiv ist, d.h. der Entropieterm kann den meist positiven Beitrag der Mischungsenthalpie nicht überkompensieren. Die abstoßenden Wechselwirkungen zwischen den einzelnen Komponenten führen zur Ausbildung geordneter Domänen (Abbildung 2-4). Aus enthalpischer Sicht ist es günstig, das Verhältnis zwischen Grenzfläche und Volumen möglichst klein zu

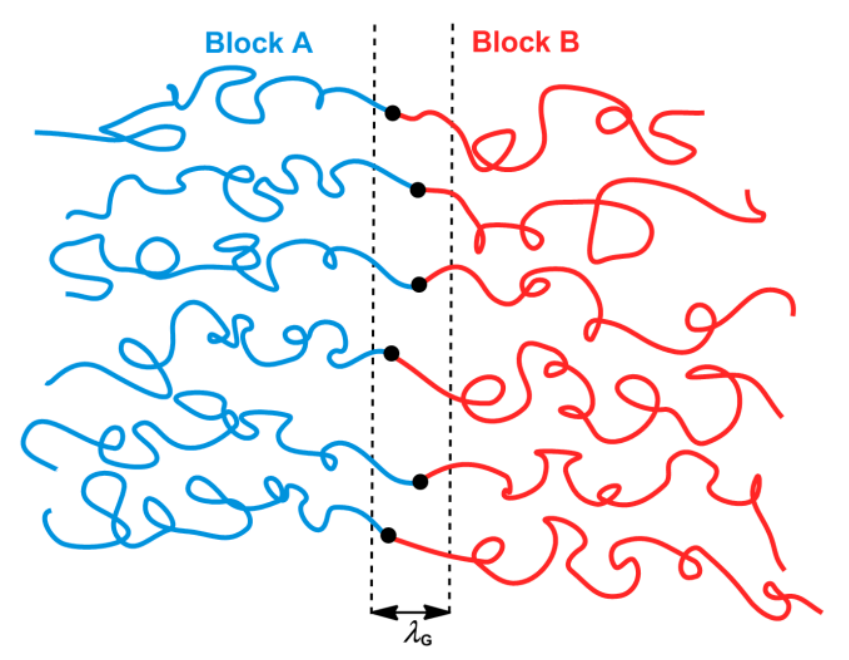

Abbildung 2-4 Grenzfläche zwischen den Blöcken A und $B$ eines Blockcopolymers. $\lambda_{G}$ beschreibt die Grenzflächendicke. 
halten und somit einen möglichst kleinen Durchmischungsbereich zu realisieren. ${ }^{80,81}$

Dem enthalpischen Effekt wirken neben der Mischungsentropie noch zwei weitere entropische Effekte entgegen. Zum einen werden die Verknüpfungspunkte zwischen den Komponenten (schwarze Punkte in Abbildung 2-4) in der Grenzfläche lokalisiert und die Anordnungsfreiheit wird im Vergleich zur homogenen Phase reduziert.80,81 Zum anderen spielt die Konformation der Ketten eine wichtige Rolle. Die Ketten werden durch die Grenzflächenminimierung gestreckt und weichen somit von der Form eines idealen Gauß'schen Knäuls mit maximaler Entropie ab.80,81 Das Wechselspiel zwischen den enthalpischen und entropischen Effekten führt im Gleichgewicht zur Ausbildung einer thermodynamisch stabilen mikrophasenseparierten Morphologie (siehe Abschnitt 2.5.2).

Das Phasenverhalten von Blockcopolymeren ist außerdem stark von der Temperatur abhängig, wie durch den oben genannten Zusammenhang mit dem Entmischungsparameter gezeigt wurde. Mit zunehmender Temperatur nimmt der Entropieterm und somit auch die Mischbarkeit der einzelnen Blöcke zu. ${ }^{82} \mathrm{Ab}$ einer bestimmten Temperatur entsteht eine homogene Phase. Dieser Übergang wird als Ordnungs-Unordnungs-Übergang (engl.: order-disorder transition, ODT) bezeichnet. Somit erhält die Temperatur, bei der dieser Übergang stattfindet, die Abkürzung $T_{\mathrm{ODT}}$.

Ein allgemeines Maß für die Unverträglichkeit zweier Polymere ist das Produkt $\chi N$. Bei dem kritischen Wert $\chi N \approx 10,5$ findet für eine symmetrische Zusammensetzung $\left(\Phi_{\mathrm{A}}=\Phi_{\mathrm{B}}=0,5\right)$ der Übergang von der ungeordneten zur geordneten Phase statt. 82 Innerhalb des geordneten Bereichs wird die MPS in zwei Regime unterteilt. ${ }^{82}$ Bei dem weak segregation limit (WSL) ist die Unverträglichkeit der Komponenten gering und das Produkt $\chi N$ liegt oberhalb von 10,5. In Abbildung 2-5 sind die Konzentrationsprofile der beiden Regime dargestellt, wobei $\Phi_{\mathrm{A}}$ den Volumenanteil der Komponente A darstellt und $x$ die Ortskoordinate. Der

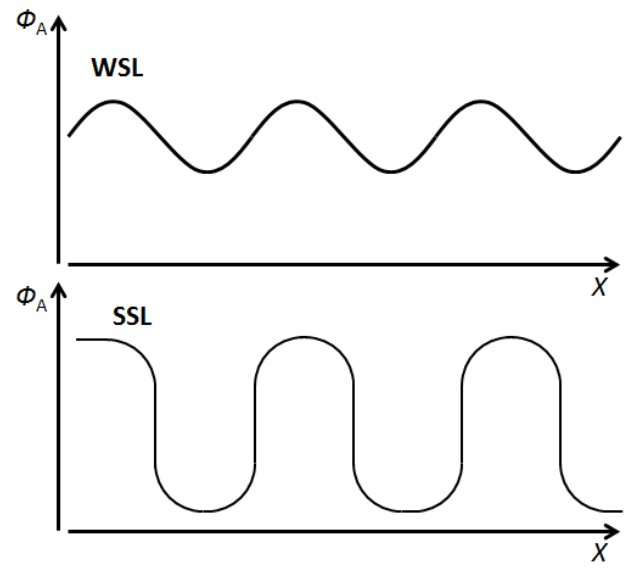

Abbildung 2-5 Konzentrationsverlauf beim weak segregation limit (oben) und beim strong segregation limit (unten). 
sinusförmige Verlauf bei dem WSL verdeutlicht eine breite Grenzfläche zwischen den Blöcken. Ist das Produkt $\chi N$ deutlich größer als 10, nimmt die Unverträglichkeit der Blöcke stark zu und dieses Verhalten wird als strong segregation limit (SSL) bezeichnet. Die Grenzfläche zwischen den Blöcken wird zunehmend dünner, was durch den kastenförmigen Verlauf (Abbildung 2-5) verdeutlicht wird. 82

Zusammenfassend kann festgehalten werden, dass drei Parameter im Wesentlichen das Phasenverhalten von Blockcopolymeren bestimmen:

- Polymerisationsgrad $N_{i}$ der Blöcke $i$

- die relativen Anteile (Volumenbrüche) $\Phi_{i}$ im Blockcopolymer

- Temperaturabhängige Entmischungsparameter $\chi$

Die Art der entstehenden Morphologie, auf die im folgenden Kapitel eingegangen wird, ist durch die Zusammensetzung des Blockcopolymers bestimmt, während die Größe der Domänen im Wesentlichen vom Polymerisationsgrad der Polymerblöcke abhängt. Beispiele für die Selbstorganisation von Blockcopolymeren erstrecken sich vom Bereich der verdünnten Lösungen (Mizellen, Vesikel), über lyotrope Phasen bei höherer Konzentration bis hin zur Substanzphase.13,29 Die gebildeten polymeren Überstrukturen können durch chemische oder physikalische Vernetzungen fixiert und somit stabilisiert werden. Die physikalische Vernetzungsreaktion resultiert aus einer Zusammenlagerung von funktionellen Gruppen oder Kettenabschnitten der Polymere ohne Ausbildung chemischer Bindungen, wie z. B. durch Wasserstoffbrückenbindungen, Einfrieren oder Unterkühlen der Polymere und sind somit thermoreversibel. Bei der chemischen Vernetzungsreaktion kommt es zu einer Ausbildung von kovalenten Bindungen wie z. B. bei der Polykondensation. Zusätzlich können chemische Vernetzungen thermisch (Radikal-Rekombination), strahlenchemisch ( $\gamma$-Strahlung) oder photochemisch $\left([2+2]\right.$-Cycloaddition) ablaufen. ${ }^{13}$ In der vorliegenden Arbeit wurde die photochemische Vernetzung verwendet und diese wird in Abschnitt 2.6 näher betrachtet.

\subsubsection{Morphologien der Mikrophasenseparation}

Eine große Anzahl verschiedener Theorien wurde entwickelt, um Aussagen zu dem Phasenverhalten und der Strukturbildung von Diblockcopolymeren zu treffen.83-89 Helfand et al. konnten im Jahr 1975 erste Berechnungen zur MPS mithilfe der Molekularfeldtheorie (engl.: mean field theory) präsentieren. ${ }^{83}$ Bei der Molekular- 
feldtheorie handelt es sich um eine Näherung aus der statistischen Physik, wodurch Systeme von miteinander wechselwirkenden Teilchen in einem Hilfsfeld zusammengefasst betrachtet werden können. Mögliche Fluktuationen durch thermische Bewegungen der Teilchen werden nicht betrachtet. Im Jahr 1980 stellte Leibler Berechnungen zum Ordnungs-Unordnungs-Übergang (engl.: orderdisorder-transition, ODT) schwach getrennter Phasen auf. ${ }^{87}$ Sowohl Helfand und Fredrickson ${ }^{90}$ als auch Leibler ${ }^{87}$ befassten sich mit dem Phasenverhalten von Blockcopolymeren im weak segregation limit. Die theoretische Beschreibung des strong segregation limit geht auf Meier ${ }^{81}$ sowie auf eine Reihe von Arbeiten von Helfand und Wasserman ${ }^{84-86}$ zurück, welche seither ständig erweitert worden. ${ }^{91,92}$ Alle entwickelten Theorien erbrachten jeweils nur im SSL oder WSL gute Näherungen für die MPS, weiterhin sagten die Theorien nicht alle experimentell gefundenen Phasen voraus. Im Jahr 1996 gelang Matsen und Schick mit ihrer erweiterten Molekularfeldtheorie der Durchbruch bei den Berechnungen von MPS bei Diblockcopolymeren.93,94 Abbildung 2-6 zeigt das von ihnen vorgestellte theoretische Phasendiagramm für Blockcopolymere, das sowohl das WSL als auch das SSL umfasst.

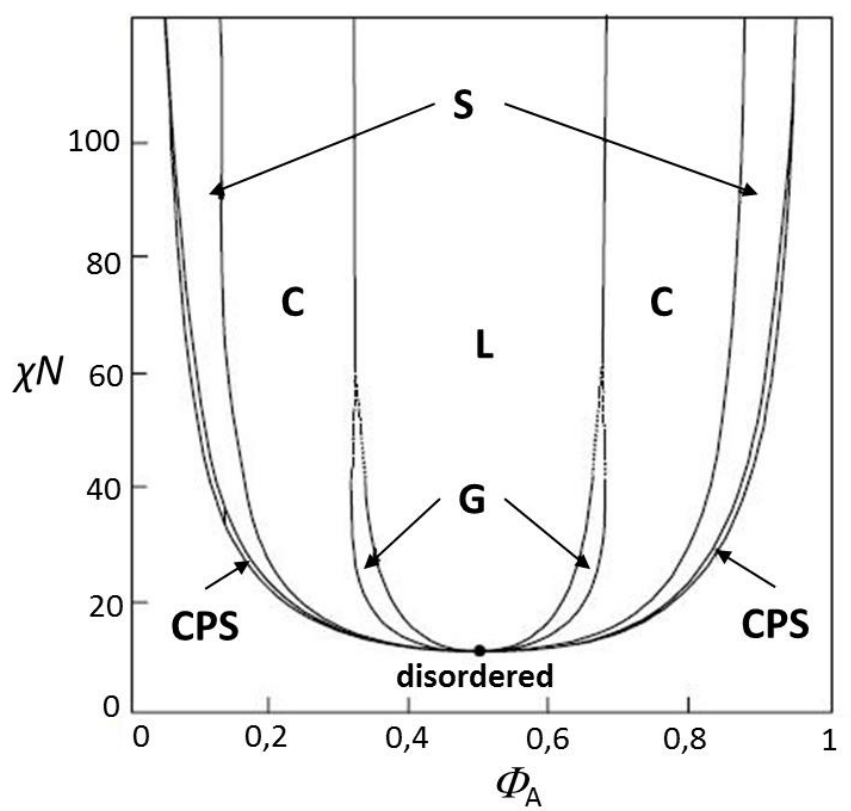

Abbildung 2-6 Theoretisches Phasendiagramm eines Diblockcopolymersystems nach Matsen und Bates, wobei die jeweiligen Abkürzungen dem Text zu entnehmen sind. ${ }^{93}$

In dem Phasendiagramm sind der Volumenbruch $\Phi_{\mathrm{A}}$ des Monomers A gegen das Produkt aus dem Polymerisationsgrad $N$ und dem Entmischungsparameter $\chi$ aufgetragen. Bei zu kleinen Werten von $\chi N$ liegt ausnahmslos ein ungeordneter 
Bereich vor (engl.: disordered). Wie bereits in Abschnitt 2.4 .1 beschrieben, entstehen mit zunehmendem Wert für $\chi N$ die geordneten Phasen. In dem Ordnungs-Ordnungs-Übergang (engl.: order-order-transition, OOT) können sich, je nach Zusammensetzung, die unterschiedlichsten Phasen ausbilden. Dazu gehören Lamellen (L), hexagonal geordnete Zylinder (C), die Gyroidphase (G), eine Phase mit raumzentrierten kubischen Sphären (S) und ein kleiner Bereich mit einer Phase hexagonal dicht gepackter Sphären (CPS). Abbildung 2-7 stellt die klassischen Strukturen von Diblockcopolymeren dar.

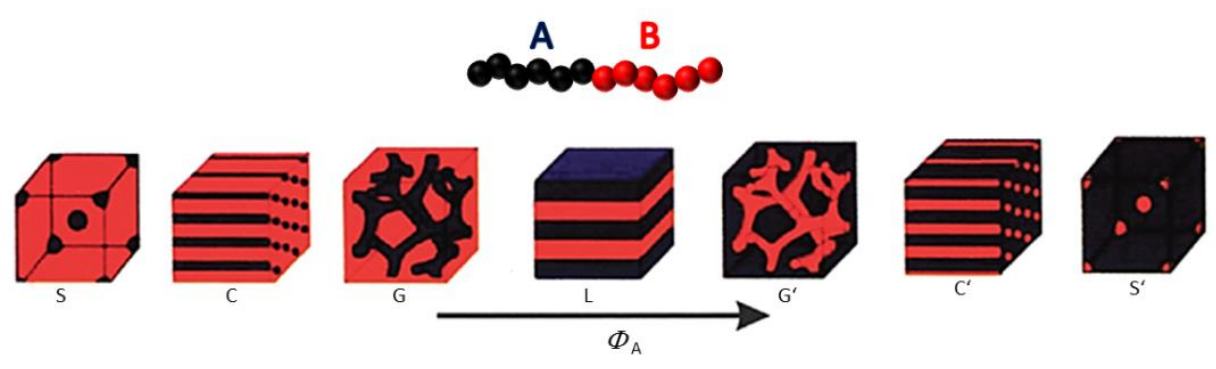

Abbildung 2-7 Klassische Morphologien der Mikrophasenseparation eines Diblockcopolymers. ${ }^{79}$ Der Anteil des Monomers A steigt von links nach rechts. Die Monomere A und B sind durch die Farben schwarz und rot dargestellt.

Abbildung 2-7 veranschaulicht sehr deutlich die Abhängigkeit der Strukturbildung der MPS von der Zusammensetzung des BCPs. Bei der MPS werden die Ketten durch die Grenzflächenminimierung gestreckt (siehe Abschnitt 2.5.1) und weichen somit von der Form eines idealen Gauß'schen Knäuls mit maximaler Entropie ab, wobei der Grad der Kettenstreckung von dem Volumenanteil $\Phi$ eines Blocks im Blockcopolymer abhängig ist. ${ }^{79}$ Abbildung 2-8 veranschaulicht die morphologischen Übergänge für den zunehmenden Volumenanteil $\Phi_{\mathrm{A}}$ von Block A (schwarz), wobei die gestrichelte Linie die Grenzfläche zwischen den Blöcken A und B darstellt. ${ }^{79}$ Dabei wird deutlich, dass sich der Block A bei einem asymmetrischen BCP, d.h. mit einem geringen Volumenanteil $\Phi_{\mathrm{A}}$ von Block $A$, in sphärischen Mikrodomänen anordnet und der Block B diese Sphären als Korona

a)

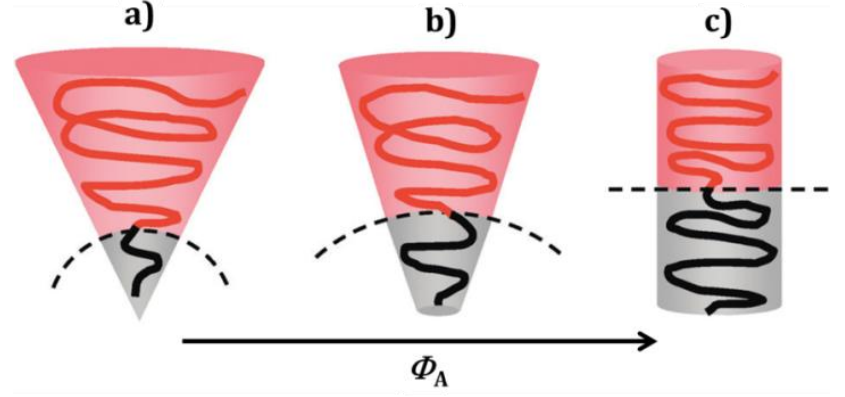

Abbildung 2-8 Mögliche Anordnungen der Polymerketten in Diblockcopolymeren: a) Sphären, b) Zylinder und c) Lamellen.79 Der Volumenanteil $\Phi_{\mathrm{A}}$ von Block A (schwarz) steigt von links nach rechts. Die gestrichelte Linie zeigt die Grenzfläche zwischen den Blöcken A und B. 
umschließt [Abbildung 2-8 a)]. Mit zunehmenden Volumenanteil $\Phi_{\mathrm{A}}$ des Blocks A ordnen sich die Polymerketten für eine Minimierung der Kettenstreckung neu an, wodurch es zu morphologischen Übergängen von Sphären über Zylinder zu Lamellen kommt. Die theoretischen Ergebnisse passen sehr gut mit dem experimentell gefundenen Phasendiagramm für das System Polystyrol-blockpolyisopren zusammen.75 Bei Blockcopolymeren mit einer höheren Anzahl an Blöcken sowie Monomerarten können sich weitere sehr komplexe Strukturen ausbilden. ${ }^{72}$

Zur Untersuchung und Beschreibung der unterschiedlichen Morphologien von Blockcopolymeren können verschiedene Messtechniken wie Kleinwinkelstreuung (engl.: Small-Angle X-ray Scattering, SAXS)95,96, Transmissionselektronenmikroskopie (engl.: Transmission Electron Microscopy, TEM) ${ }^{97,98}$, RasterElektronen-Mikroskopie (engl.: Scanning Electron Microscopy, SEM) ${ }^{99}$ und Rasterkraftmikroskopie (engl.: Atomic Force Microscopy, AFM) ${ }^{100}$ verwendet werden. In der vorliegenden Arbeit wurden die Blockcopolymere mittels Rasterkraftmikroskopie analysiert. Die Technik des AFMs wird im Abschnitt 2.6 näher erläutert.

\subsubsection{Weitere Einflussfaktoren bei der Mikrophasenseparation}

Seit der Entwicklung des ersten Blockcopolymers Mitte der 1950er Jahre durch Szwarc ist das Interesse an diesem Thema stetig gestiegen. ${ }^{11}$ Besonders die Selbstorganisation (engl.: self-assembly) von BCP bietet ein breites Anwendungsgebiet von Wirkstofftransport bis hin $\mathrm{zu}$ optischen Datenspeichern. ${ }^{33}$ Umso wichtiger ist die Entwicklung und Forschung auf dem Gebiet der Selbstorganisation von BCP geworden. Das Phasenverhalten von BCP wird im Wesentlichen von drei Faktoren beeinflusst, wie bereits in Abschnitt 2.5.1 beschrieben.

Perrier et al. konnten bei der Untersuchung von PMMA- $b$-PBA nicht nur eine Abhängigkeit der Strukturbildung von der Zusammensetzung des BCP-Systems feststellen, sondern auch die Schichtdicke der Polymerfilme ist von entscheidender Bedeutung bei der Orientierung der BCP.101 Weiterhin konnte festgestellt werden, dass durch die verschiedenen Polaritäten der Blöcke ein unterschiedliches Benetzungsverhalten des Substrats hervorgerufen wird, wodurch wiederum die Ausrichtung der einzelnen Blöcke beeinflusst wird. Durch Vorbehandlung des 
Substrates kann jedoch das Benetzungsverhalten des BCP beeinflusst werden. ${ }^{101,102}$

Ein weiterer wichtiger Einflussfaktor bei der Ausbildung der MPS ist das Tempern (engl.: annealing) des Blockcopolymers. Stehlin et al. stellten bei der Untersuchung von PS- $b$-PMMA fest, dass durch Erhöhung der Temperatur die Ausrichtungszeit der BCP beeinflusst wird.103 Die Erwärmung der BCP über ihre Glasübergangstemperatur $T_{\mathrm{g}}$ erhöht die Kettenbeweglichkeit, wodurch sich die jeweiligen Strukturen erst anordnen können. Rasmont et al. untersuchten unterschiedliche (Meth)Acrylat-BCP und fanden heraus, dass sich nach einer bestimmten Zeit das thermodynamische Gleichgewicht eingestellt hat und die bestmögliche Anordnung der einzelnen Blöcke erreicht wurde.104 Rasmont beobachtete auch eine Beeinflussung der Orientierung der BCP bei der Wärmebehandlung der BCP in einer Umgebung von Lösungsmitteldampf. ${ }^{104}$

Wie bereits festgestellt, ist das Phasenverhalten von BCP stark abhängig von der Temperatur. Dabei zeigt der temperaturabhängige Entmischungsparameter $\chi$ einen wichtigen Einfluss auf die Ausbildung der MPS, da ab einer kritischen Temperatur nur noch eine homogene Phase vorliegt, auch bezeichnet als $T_{\text {ODT. }}$ Zahlreiche Polymersysteme wie PS-PMMA ${ }^{105}$, SBS-SIS ${ }^{74}$ oder PS-PI106,107 wurden auf ihren order-disorder-Übergang untersucht, wobei eine Abhängigkeit der TODT von der einzelnen Blocklänge und der Molmasse des Polymers festgestellt wurde. Zhao et al. berichteten von einer Beeinflussung der $T_{\text {ODT }}$ durch den Einbau von Palladium Nanopartikeln in Nanokomposit-Materialien bestehend aus PSPMMA. ${ }^{108}$ Im Abschnitt 4.4 und 4.5 folgt die Untersuchung unterschiedlicher Einflussfaktoren auf das Phasenverhalten der in dieser Arbeit synthetisierten BCP.

\subsubsection{Anwendung von Blockcopolymeren}

Die RDRP bietet mit Methoden wie z.B. ATRP, NMP und RAFT-Polymerisation eine Vielzahl von Möglichkeiten der Synthese von Blockcopolymeren unterschiedlichster Architekturen, die wiederum zahlreiche technische und medizinische Anwendungen finden. So werden amphiphile Blockcopolymere als polymere Detergentien, Phasenvermittler sowie Stabilisatoren für kolloidale Dispersionen oder in Membranen verwendet. ${ }^{14,76}$ Besonders funktionalisierte und strukturierte Blockcopolymere wie Biomaterialien finden Einsatz als Implantate, beim Wirkstofftransport, ${ }^{13}$ sowie in der Gentherapie. ${ }^{33}$ Ein weiteres Anwendungsfeld ist die Herstellung von anorganischen Nanohybridmaterialien wie z.B. Metallhybride, 
Halbleiterhybride und Biominerale. ${ }^{13}$ Technische Nutzung finden Blockcopolymere als Nano-Schablonen, sog. Template, ${ }^{14}$ optische Schaltsensoren ${ }^{109}$ und optische Datenspeicher. ${ }^{33,110}$

\subsection{Dynamisch mechanische Analyse}

Die dynamisch mechanische Analyse (DMA) wird angewendet, um die mechanischen und viskoelastischen Eigenschaften von Materialien als Funktion von Temperatur, Zeit und Frequenz zu bestimmen. Dabei wird an die Materialprobe eine sinusförmige Kraft angelegt und die Deformation des Materials wird gemessen. Mit der DMA werden die Kraftamplitude, die Verformungsamplitude und deren Phasenverschiebung $\Delta \varphi$ gemessen. Aus den Messungen kann der Elastizitätsmodul $E$ und der Verlustfaktor $\tan \delta$ bestimmt werden. Der Elastizitätsmodul setzt sich aus dem Speichermodul $E^{\prime}$ und Verlustmodul $E^{\prime \prime}$ zusammen. Der Speichermodul ist hierbei ein Maß für die Speicherung der Energie und der Verlustmodul beschreibt die vom Material dissipierte Energie. Das Verhältnis von Verlust- und Speichermodul stellt den Verlustfaktor $\tan \delta$ dar, welcher ein Maß für die Dämpfung ist. Die Probe muss sich während der Beanspruchung die gesamte Zeit im Hooke'schen Bereich (linearelastisch) befinden. ${ }^{111}$

Das Verhalten der Materialprobe kann bei der Krafteinwirkung in drei Bereiche unterteilt werden:

1. Rein elastisches Verhalten: Hierbei reagiert die Probe ohne zeitliche Verzögerung auf die einwirkende Kraft und die Probe verhält sich wie ein idealer Festkörper. Der Phasenwinkel beträgt $\Delta \varphi=0^{\circ}$.

2. Rein viskoses Verhalten: Hierbei wird die gesamte Energie dissipiert (in Wärme umgewandelt) und die Probe verhält sich wie eine ideale Flüssigkeit. Der Phasenwinkel beträgt $\Delta \varphi=90^{\circ}$.

3. Viskoelastisches Verhalten: Die Verformung der Probe folgt der Kraft mit einer gewissen Phasenverschiebung im Bereich von $0^{\circ}<\Delta \varphi<90^{\circ}$. Polymere reagieren viskoelastisch und liegen zwischen dem Grenzfall aus 1. und 2.

Mit der DMA kann weiterhin die Glasübergangstemperatur $T_{\mathrm{g}}$ von Polymeren untersucht werden. Am Glasübergang werden die Polymerketten beweglicher und dissipieren in hohem Maße Energie. Dies äußert sich in einem Abfallen des 
Speichermoduls $E^{\prime}$, wohingegen der Verlustmodul $E^{\prime \prime}$ und $\tan \delta$ ein Maxima durchlaufen. In der vorliegenden Arbeit wurde mittels DMA der globale Elastizitätsmodul unterschiedlicher Blockcopolymere bestimmt sowie deren Glasübergangstemperaturen. Die DMA-Messungen wurden in Kooperation mit dem Institut der Physik von Stefanie Finkhäuser durchgeführt. Die detaillierte Durchführung der Experimente wird im Abschnitt 6.6.5 beschrieben.

\subsection{Rasterkraftmikroskopie}

Die Rasterkraftmikroskopie (engl.: Atomic Force Microscopy, AFM) wurde erst 1986 von Binnig, Quate und Gerber als Nachfolger der Rastertunnelmikroskopie (scanning tunneling microscopy, STM) erfunden. ${ }^{112}$ Bei dem STM wird durch die ortsaufgelöste, abstandsabhängige Messung eines Tunnelstroms die Oberfläche leitender Materialien in atomarer Auflösung abgebildet. Der Tunnelstrom verläuft zwischen der Messsonde und der Probenoberfläche. Mit dem AFM wird im Gegensatz zum STM die Kraft, die in unmittelbarer Nähe zur Oberfläche auf die Sonde wirkt, gemessen. Das AFM ermöglicht die Messung nichtleitender Oberflächen, wodurch sich eine größere Vielfalt an Anwendungsgebieten bietet. Das AFM wurde in den letzten Jahren zu einem unentbehrlichen Instrument, das die nanoskalige Oberflächenuntersuchung verschiedenster Proben, wie z. B. organische und biologische Substanzen, ermöglicht. Die AFM-Untersuchungen sind an der Luft, im Vakuum sowie im flüssigen Medium durchführbar. Darüber hinaus kann das AFM als sehr präzises Instrument zur Messung mechanischer Eigenschaften wie Elastizität und Härte verwendet werden.113-116

\subsubsection{Messprinzip}

Eine feine Spitze (engl.: tip), die an der Unterseite eines mikroskopisch kleinen Federbalkens, dem sog. Cantilever angebracht ist, wird rasterförmig über die Probenoberfläche geführt. Abbildung 2-9 stellt den Aufbau des AFMs dar.

Die zu untersuchende Probe befindet sich auf einem in $x y z$-Richtung beweglichen Probentisch, der über piezoelektrische Elemente gesteuert wird. Die Messgröße am Messkopf ist die direkte Kraftwechselwirkung zwischen Spitze und Probenoberfläche. Die Probe wird in x- und y- Richtung bewegt. Beim Abrastern der Oberfläche können die auftretenden Unebenheiten Kräfte auf den Cantilever ausüben, wodurch sich dieser verbiegt. Der auf das Ende des Cantilevers 
fokussierte Laserstrahl wird auf einen positionsempfindlichen Photodetektor reflektiert. Ändert sich nun die Verbiegung, dann ändert sich auch der Einfallswinkel und der Laserpunkt wandert auf dem Photodetektor. Der Photodetektor besteht wiederum aus vier Photodioden, gekennzeichnet durch vier Quadranten in Abbildung 2-9. Die Photodioden wandeln das einfallende Licht in eine Spannung um. Über die Spannungsdifferenz zwischen den oberen und unteren Dioden kann die vertikale Verschiebung des Laserstrahls und somit die vertikale Verschiebung des Cantilevers bestimmt werden. Die Bestimmung der horizontalen Laserstrahlverschiebung erfolgt über die Spannungsdifferenz der rechten und linken Dioden. Der Cantilever besteht meist aus Silizium oder Siliziumnitrid. Die Wahl eines geeigneten Cantilevers ist abhängig von der Messmethode und der zu untersuchenden Probe. Die Cantilevermodelle können z. B. in der Federkonstante, der Frequenz und dem Spitzenradius variieren.

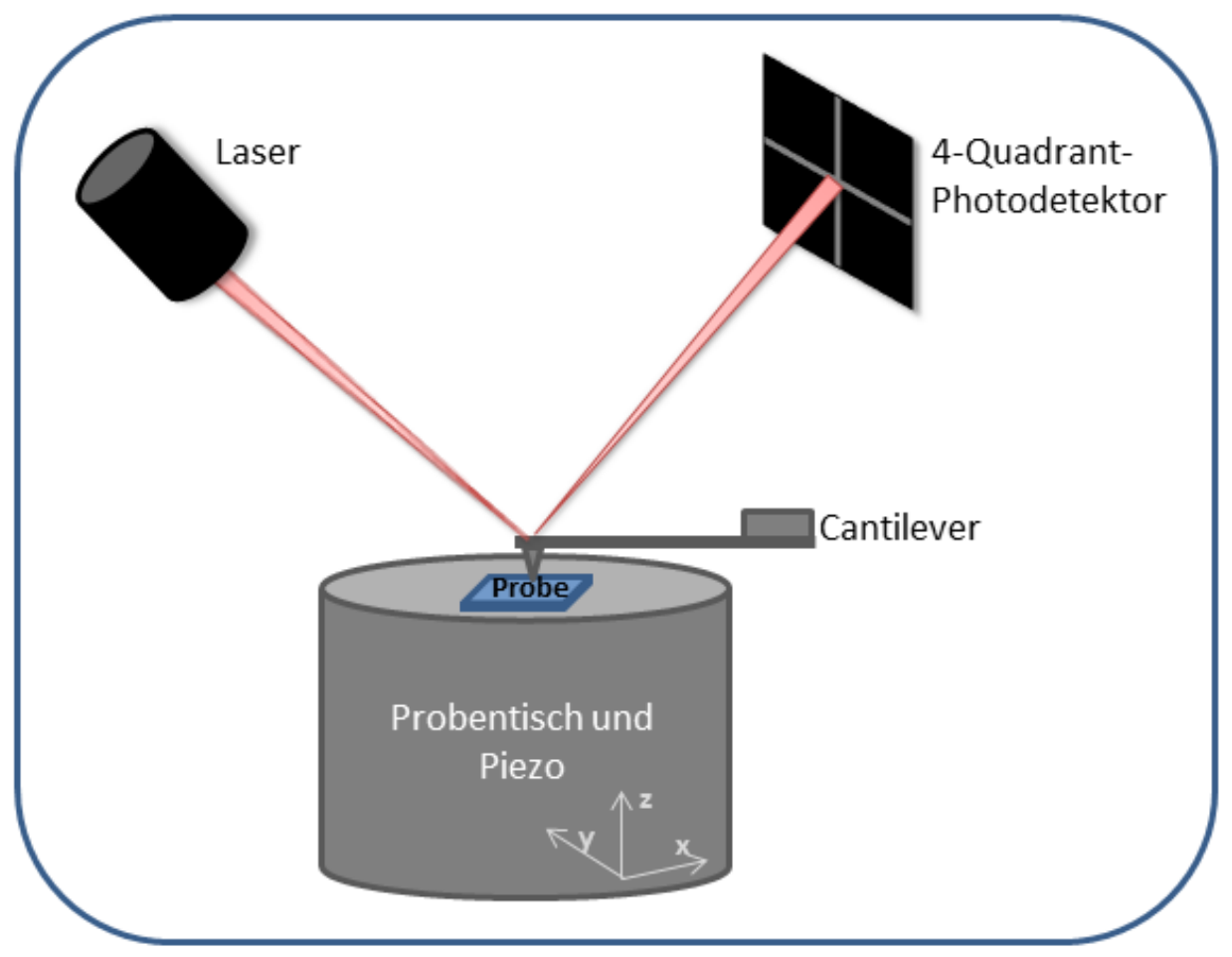

Abbildung 2-9 Schematischer Aufbau eines AFMs: Die Probe ist auf einem in xyz-Richtung beweglichen Probentisch angebracht. Der Laserstrahl wird auf die Cantilverspitze ausgerichtet, wobei der durch das Abrastern der Probe veränderte Strahl mit Hilfe des Vier-QuadrantPhotodetektors registriert wird.

Die Verbiegung des Cantilevers $\delta$ ergibt sich aus der gemessenen Spannungsdifferenz $\Delta U$ des Photodetektors und der Deflection Sensitivity $S_{\mathrm{D}}$, die einen 
Umrechnungsfaktor darstellt, der normalerweise durch eine Kalibration des Detektors bestimmt wird.

$$
\delta=S_{\mathrm{D}} \cdot \Delta U
$$

Zur Berechnung der Kraft $F$ aus der Cantileververbiegung $\delta$ muss die Federkonstante des Cantilevers $k_{c}$ bekannt sein. Mit dieser Voraussetzung ist die Kraft durch das Hooke'schen Gesetz gegeben:

$$
F=k_{\mathrm{c}} \cdot \delta
$$

\subsubsection{Betriebsmodi}

Das AFM kann in verschiedenen Modi betrieben werden. Die wohl bekanntesten Modi sind in Abbildung 2-10 dargestellt, wobei die Firma Bruker einen neuen Modus (PeakForce Tapping ${ }^{\mathrm{TM}}$ ) entwickelt hat, der eine Kombination aus Contact Mode und Tapping Mode ${ }^{\mathrm{TM}}$ bildet.

Contact Mode:117,118 Bei diesem Modus berührt die Spitze des Cantilevers die Probenoberfläche und die Krafteinwirkung auf den Cantilever bleibt durch Anpassen der z-Achse stets gleich. Diese Methode wird vor allem für harte und flache Proben verwendet. Der Modus lässt sich schnell durchführen. Allerdings können die Proben durch laterale Kräfte beschädigt werden. Schwach adsorbierte Teile der Probe können durch die Spitze verschoben werden und weiche Proben können durch große Auflagekraft beschädigt werden.

Tapping Mode $e^{\mathrm{TM}}: 118,119$ Bei diesem Modus wird der Cantilever nahe seiner Resonanzfrequenz angeregt und schwingt somit über die Probenoberfläche, wobei er nur sehr kurz die Oberfläche berührt. Bildlich gesprochen „klopft“ (engl.: tap) die Spitze die Probenoberfläche ab, wie in Abbildung 2-10 schematisch dargestellt. Durch die kurze Auflagekraft wird die Probe geschont und Messungen von weichen Proben (Polymere, Zellen) können durchgeführt werden. 
PeakForce Tapping ${ }^{\mathrm{TM}}$ :118,120 Bei diesem Modus wird im Gegensatz zum Tapping $M_{o d} e^{\mathrm{TM}}$ der Cantilever unterhalb seiner Resonanzfrequenz angeregt. Dadurch kann beim PeakForce Tapping ${ }^{\text {TM }}$ die Maximalkraft (engl.: peak force) auf die Spitze kontrolliert werden. Der Cantilever dringt hierbei in die Probenoberfläche ein. Allerdings wird z. B. für die Untersuchung eines Höhenprofils die Kontaktfläche zwischen Spitze und Probe minimiert. Dadurch werden die Spitze und die Probe geschont. Durch einen Heran- und WegfahrZyklus des Cantilevers wird für jeden Bildpunkt eine vollständige Kraft-AbstandsKurve aufgenommen. Aus dieser Kurve können nicht nur topologische Informationen der Probe bestimmt werden, sondern auch mechanische Eigenschaften wie Elastizitätsmodul, Adhäsion, Deformation und Energiedissipation. Allerdings muss der Cantilever für die Bestimmung mechanischer Eigenschaften tief in die Probe eindringen, sodass für diese Messungen mehr Kraft auf die Spitze ausgeübt wird. Im folgenden Unterkapitel wird dieser spezielle Modus genauer betrachtet.

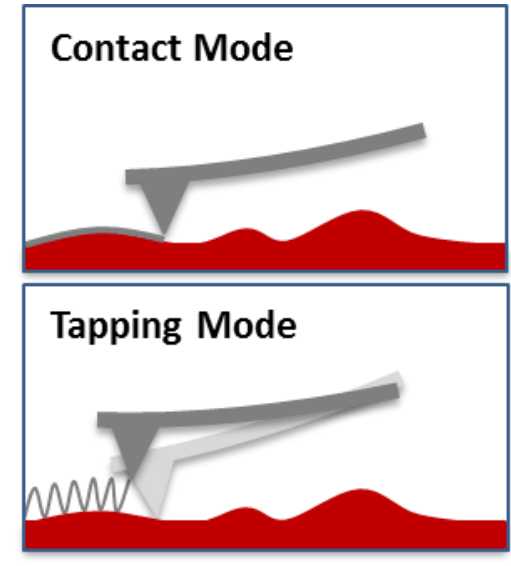

Abbildung 2-10 Schematische Darstellung zweier Betriebsmodi eines AFMs: der Contact Mode und der Tapping Mode ${ }^{\mathrm{TM}}$.

\subsubsection{Bestimmung mechanischer Eigenschaften mittels AFM}

Um auf nanoskopischer Skala die mechanischen Eigenschaften der Probe bestimmen zu können, hat Bruker den Modus PeakForce-QNM ${ }^{\mathrm{TM}}$ (engl.: PeakForce Quantitativ Nanomechanical Mapping ${ }^{\mathrm{TM}}$ ) entwickelt. Die beim PeakForce Tapping ${ }^{\mathrm{TM}}$ aufgenommenen Heran- und Wegfahr-Kurven des Cantilevers werden beim PeakForce-QNM ${ }^{\mathrm{m}}$ in Echtzeit analysiert und die unterschiedlichen mechanischen Eigenschaften der Probe werden für jeden abgerasterten Punkt berechnet. In Abbildung 2-11 a) wird die Verbiegung des Cantilevers in Abhängigkeit vom Probenabstand dargestellt. Die unterschiedlichen Kräfte zwischen dem Cantilever und der Oberfläche werden in Abbildung 2-11 b) verdeutlicht.

Die Punkte 1 - 6 in der Heran- und Wegfahrkurve (Abbildung 2-11 a)) werden für ein besseres Verständnis genauer erläutert. Bei größeren Entfernungen sind die Kräfte zwischen Cantilever und Probe noch verschwindend gering, wodurch der Cantilever noch nicht verformt wird (Punkt 1). Beim Heranfahren des Cantilevers an die Probe finden attraktive Wechselwirkungen (van-der-Waals-Kräfte) statt 
und der Cantilever wird dabei nach unten gebogen (Punkt 2). Mit zunehmender Abstandsverringerung springt der Cantilever an die Probenoberfläche (Punkt 3), wodurch sich die Verbiegung schlagartig verstärkt. Mit zunehmender Krafteinwirkung wird der Cantilever in die Probe gedrückt und biegt sich dabei in die entgegengesetzte Richtung (Punkt 4-5). Die auf den Cantilever einwirkende Kraft (engl.: PF setpoint) kann über das AFM-Programm manuell oder automatisch eingestellt werden. Am Punkt 5 ist der Heranfahrvorgang beendet. Beim anschließenden Wegfahrvorgang nimmt die Verbiegung des Cantilevers ab und kehrt sich am Punkt 4 wieder um. Durch Adhäsionskräfte haftet der Cantilever an der Probe und springt erst zu einem späteren Zeitpunkt als beim Heranfahren von der Probe weg (Punkt 6). Nun befindet sich der Cantilever wieder in der Ausgangsposition.

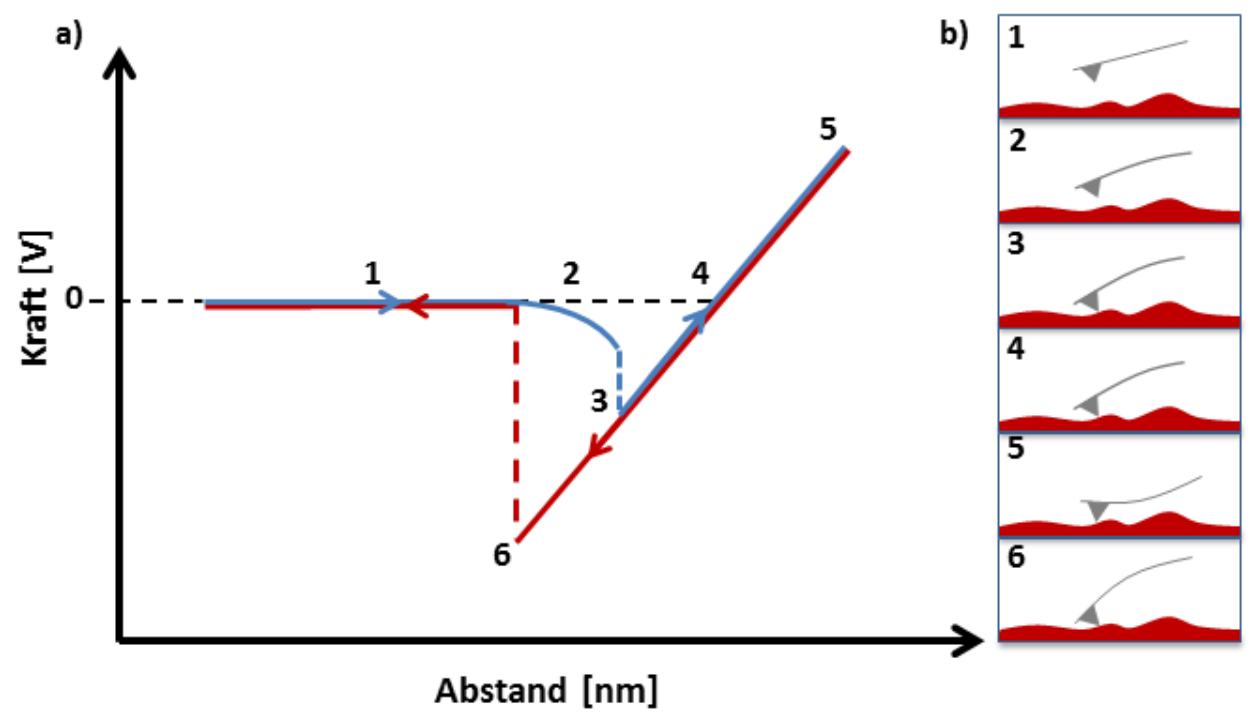

Abbildung 2-11 a) Cantileververbiegung in Abhängigkeit vom Piezoversatz für das Heranfahren (blau) und Wegfahren (rot) an die Oberfläche; b) schematische Abbildung des Cantilevers an markanten Punkten im Diagramm.

Die Bestimmung der mechanischen Eigenschaften wie z. B. des Elastizitätsmoduls erfolgt aus der Kraft-Abstands-Kurve. Der typische Verlauf einer Kraft-AbstandsKurve ist in Abbildung 2-12 dargestellt.

Der Elastizitätsmodul der Probe $E_{\mathrm{DMT}}$ kann nach dem Modell von Derjaguin-MullerToporov (DMT) aus der Wegfahrkurve bestimmt werden. Dafür wird Gleichung (18) an den Anfangsbereich der Wegfahrkurve (grün markierte Linie in Abbildung 2-12) angepasst, um zunächst den reduzierten E-Modul $E_{\mathrm{r}}$ zu ermitteln. ${ }^{114}$ 


$$
F_{\text {tip }}=\frac{4}{3} E_{\mathrm{r}} \sqrt{R \cdot d^{3}}+F_{\mathrm{adh}}
$$

Dabei sind $F_{\text {tip }}$ die Kraft, die auf die Spitze des Cantilevers einwirkt, $R$ der Spitzenradius, $d$ der Spitzen-Oberflächen-Abstand und $F_{\text {adh }}$ die Adhäsionskraft.

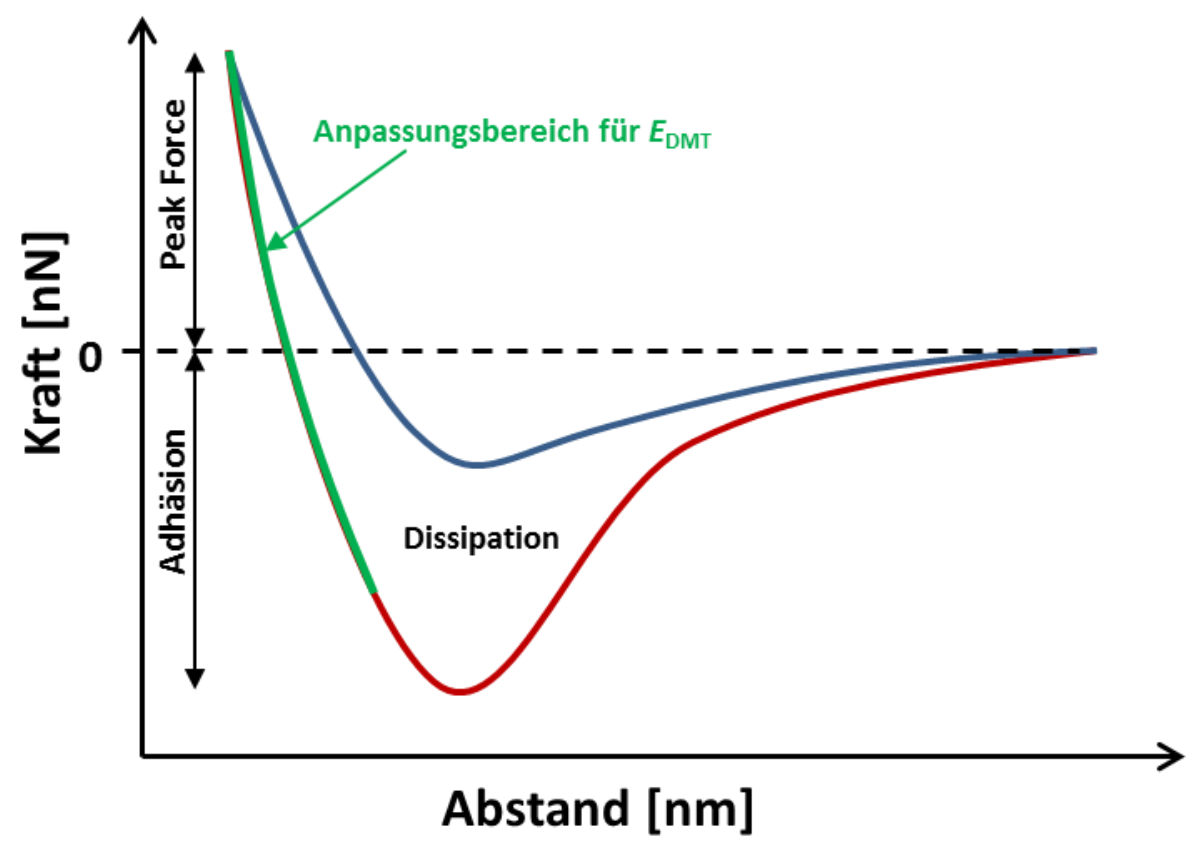

Abbildung 2-12 Kraft-Abstands-Kurve beim Heran- (blau) und Wegfahren (rot) eines Cantilevers von der Probenoberfläche.

Mit Hilfe des Zusammenhangs ${ }^{121}$ :

$$
\frac{1}{E_{\mathrm{r}}}=\frac{1-v_{\text {Probe }}^{2}}{E_{\mathrm{DMT}}}+\frac{1-v_{\mathrm{Tip}}^{2}}{E_{\mathrm{Tip}}}
$$

kann der tatsächliche E-Modul der Probe $\left(E_{\mathrm{DMT}}\right)$ bestimmt werden, wobei $E_{\text {Tip }}$ der E-Modul der Spitze und $v_{\text {Tip }}$ die Poissonzahl der Cantileverspitze sind. Mit der Annahme, dass sich die Spitze des Cantilevers nicht verformt und unendlich hart ist, geht der zweite Term gegen Null. Die Poisson-Zahl für Polymere ( $\left.v_{\text {Probe }}\right)$ liegt in dem Bereich 0,2 bis 0,5.121 Mit diesen Annahmen kann der E-Modul der Probe $E_{\mathrm{DMT}}$ nach folgender Gleichung bestimmt werden:

$$
E_{\mathrm{DMT}}=E_{\mathrm{r}}\left(1-v_{\mathrm{s}}^{2}\right)
$$


Das Minimum der Wegfahrkurve (siehe Abbildung 2-12) entspricht der Adhäsionskraft und die Fläche zwischen den Kurven der Energiedissipation. Es gilt:121

$$
W_{\text {diss }}=\int \vec{F} \mathrm{~d} \vec{z}=\int_{0}^{t_{\text {ges }}} \vec{F} \vec{v} \mathrm{~d} \vec{t}
$$

Dabei sind $\vec{F}$ der Kraftvektor und d $\vec{z}$ der Verschiebungsvektor, der sich wiederum aus dem Produkt der Geschwindigkeit $\vec{v}$ der Spitze und des zeitlichen Verlaufs $\mathrm{d} \vec{t}$ über die Gesamtdauer einer Messperiode $t_{\text {ges }}$ ergibt. Dabei beschreibt eine Messperiode die Dauer für das Heran- und Wegfahren des Cantilevers. Um verlässliche Daten ermitteln zu können, wird das System an einem Standard mit bekannten E-Moduln kalibriert. ${ }^{122}$ Die Kalibrierung wird in Abschnitt 6.6.3 genauer erläutert.

\subsubsection{Bildanalyse mit Hilfe der Leistungsspektraldichte-Funktion}

Zur Beschreibung der nanostrukturierten Oberfläche wird die Leistungsspektraldichte-Funktion (engl.: Power Spectral Density, PSD) verwendet. Die PSD-Funktion ist das Betragsquadrat des Fourierspektrums der Oberflächentopographie und enthält Informationen über die vertikale und laterale Verteilung einzelner Strukturanteile.

Die Berechnung der PSD kann entweder eindimensional aus aufgenommenen Profildaten $(1 D-P S D)^{123}$ oder zweidimensional aus Flächendaten $(2 D-P S D)^{124}$ erfolgen. Die Oberflächentopographie einer Probe kann als eine zweidimensionale Funktion (horizontal und vertikal) aufgefasst werden, die mit Hilfe der Fast Fourier-Transformation (FFT) ${ }^{125}$ in ihre Raumwellen bzw. Ortsfrequenzen zerlegt werden kann. Gleichung (22) beschreibt die Berechnung der FFT $Z\left(f_{x}, f_{y}\right)$ aus den erfassten Daten eines zweidimensionalen Höhenprofils $z(x, y):{ }^{126}$

$$
Z\left(f_{x} f_{y}\right)=\int_{-L_{x} / 2}^{L_{x} / 2} \int_{-L_{y} / 2}^{L_{y} / 2} z(x, y) \cdot \exp \left(-i 2 \pi\left(f_{x} x+f_{y} y\right) \cdot \mathrm{d} x d y\right.
$$

wobei $L_{x}$ und $L_{y}$ die Gesamtmessstrecken (Höhe und Breite) des Höhenprofils $z(x, y), i=(-1)^{1 / 2}, x$ und $y$ die Oberflächenpositionen in horizontaler und vertikaler Richtung und $f_{x}$ und $f_{y}$ die Ortsfrequenzen sind. Die Ortsfrequenz bezeichnet bei örtlich periodischen Vorgängen den Kehrwert der räumlichen Wellenlänge $\lambda$ und wird wie folgt definiert: 


$$
k=|\vec{k}|=\frac{2 \pi}{\lambda}=2 \pi \cdot f,
$$

wobei $k$ die Wellenzahl und $\vec{k}$ der Wellenvektor sind. Daraus ergibt sich, dass große Strukturen mit einer großen Wellenlänge einen kleinen Wellenvektor bzw. eine kleine Ortsfrequenz besitzen und umgekehrt. Weiterhin gibt die Ausrichtung der Strukturen die Richtung der Wellenzahl an, die positiv oder negativ sein kann. Das Betragsquadrat von $Z\left(f_{x}, f_{y}\right)$ ergibt die Leistungsspektraldichte und wird in folgender Gleichung beschrieben:124

$$
\operatorname{PSD}\left(f_{x}, f_{y}\right)=\frac{\left|Z\left(f_{x} f_{y}\right)\right|^{2}}{A}
$$

wobei A die Fläche des Höhenprofils ist, die sich aus den Messstrecken $L_{\mathrm{x}}$ und $L_{\mathrm{y}}$ zusammensetzt. Mit der FFT lässt sich die Vorzugsrichtung der mit Hilfe des AFMs ermittelten Strukturen bestimmen, wodurch die FFT zu einem wichtigen Anwendungselement bei der Bildanalyse wird. Die Auswertung der AFMHöhenprofile erfolgt mit der 2D-PSD-Funktion der NanoScope Analysis-Software und die Oberflächentopographie wird der Auftragung der logarithmischen Leistunsspektraldichte gegen die Ortsfrequenz entnommen.

\subsection{Photochemische Vernetzung}

Wie in Abschnitt 2.5.1 bereits erwähnt, existiert eine Vielzahl an Vernetzungsmöglichkeiten für Polymere, wobei die photochemische Vernetzung an zunehmender Bedeutung gewinnt. Für diese Reaktion müssen vernetzungsfähige Gruppen, entweder im Polymerrückgrat oder in der Seitenkette, in das Polymer eingebaut werden. Monomere, die zu einer photochemischen Vernetzung führen können, bestehen im Allgemeinen aus einer UV-aktiven Einheit und einer Monomereinheit, an der die Polymerisation stattfindet. Die ersten Arbeiten zu synthetischen lichtempfindlichen Polymeren wurden im Jahr 1959 von Minsk et al. veröffentlicht, worin sie die photochemische Dimerisierung des Polyvinylcinnamats beschreiben. ${ }^{127-129}$ Neben den Polyvinylcinnamaten ${ }^{130,131}$ gibt es auch noch andere zahlreiche photoreaktive Gruppen wie z.B. die Acridizinium-Salze,132 die 2- und 4Styrylpyridinium- Salze, ${ }^{133,134}$ die Dimethylmaleinimid- Gruppe ${ }^{135,136}$ und die Coumarin- Derivate. ${ }^{127}$ Für die Vernetzung verwendet man im Allgemeinen Licht im UV- und sichtbaren Spektralbereich von 250 bis $450 \mathrm{~nm}$. Die UV-Vernetzung 
von Polymeren findet Anwendung für Oberflächenbeschichtungen, Druckfarben, Zahnfüllungen, gedruckte Schaltkreise für elektronische Ausrüstungen und für polymere Photolacke.18 Zum Aufbau photovernetzbarer Polymere wurden in dieser Arbeit Coumarin-Derivate als UV-aktive Einheit verwendet. Die Photochemie der Coumarine wird in den folgenden Abschnitten genauer betrachtet, da ein wichtiger Bestandteil dieser Arbeit die Vernetzung einzelner Blöcke der mittels RAFT-Polymerisation hergestellten BCP ist.

\subsubsection{Coumarin und seine Anwendungsgebiete}

In den 1820er Jahren wurde Coumarin erstmals aus der Tonkabohne isoliert und inzwischen sind mehr als 1000 Coumarin-Derivate entwickelt worden.127,137 Coumarin und seine Derivate werden vielfältig verwendet, beispielsweise als Duftstoffe in Parfüm-, Kosmetik- und Hygieneprodukten oder als Zusatzstoff in Tabak. Aus toxikologischer Sicht ist jedoch anzumerken, dass isoliertes Coumarin im Tierversuch leberschädigend (hepatoxisch) wirkte und somit keineswegs unbedenklich ist.137,138 Ein weiteres wichtiges Anwendungsgebiet der CoumarinDerivate stellt die Medizin dar, wo die Wirksamkeit einiger Coumarine gegen HIViren im Fokus der Forschung liegt.139,140 Seit den 1940er Jahren wurden die Coumarin-Komponenten auf ihre Einstellbarkeit der Absorptions- und Fluoreszenzeigenschaften durch Substitution am Coumaringrundgerüst (siehe Abbildung 2-13),141 Variation des Lösungsmittels ${ }^{142}$ sowie des pH-Wertes $^{143}$ untersucht. Aufgrund der hohen Fluoreszenzausbeute werden die CoumarinDerivate als Laserfarbstoffe und in organischen Leuchtdioden (OLEDs) eingesetzt.142,144,145 Eine der wichtigsten Eigenschaften der Coumarine ist die photochemisch herbeigeführte Dimerisierung und die Dimerspaltung, die in folgendem Abschnitt diskutiert

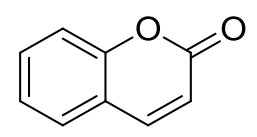

Abbildung 2-13 Darstellung des Coumaringrundgerüsts. werden.

\subsubsection{Photodimerisierung und Dimerspaltung}

Die Photodimerisierung von Coumarin wurde im Jahr 1902 von Ciamician und Silber entdeckt. ${ }^{146}$ Doch erst rund 60 Jahre später kamen Schenk et. al mit Hilfe der NMR-Spektroskopie zu der Erkenntnis, dass bei der Bestrahlung von Coumarinstrukturen Isomere mit unterschiedlichen physikalischen Eigenschaften entstehen. ${ }^{147}$ Dabei kann es zur Bildung von vier möglichen regio- und 
stereoisomeren Dimerstrukturen kommen, wobei die genauen Reaktionsmechanismen bis heute kontrovers diskutiert werden. ${ }^{147-151}$ Die photochemische Dimerisierung ist eine photo-reversible [2+2]-Cycloaddition, die bei Bestrahlung mit UV-Licht $(\lambda>310 \mathrm{~nm})$ stattfindet (Schema 2-6).

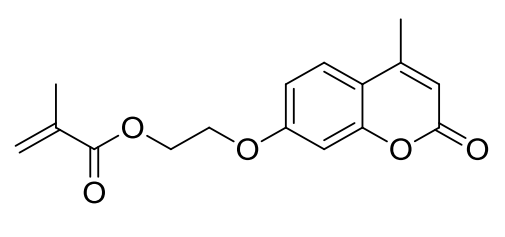

7-(2-Methacryloyloxyethoxy)-4-methylcoumarin (CMA)

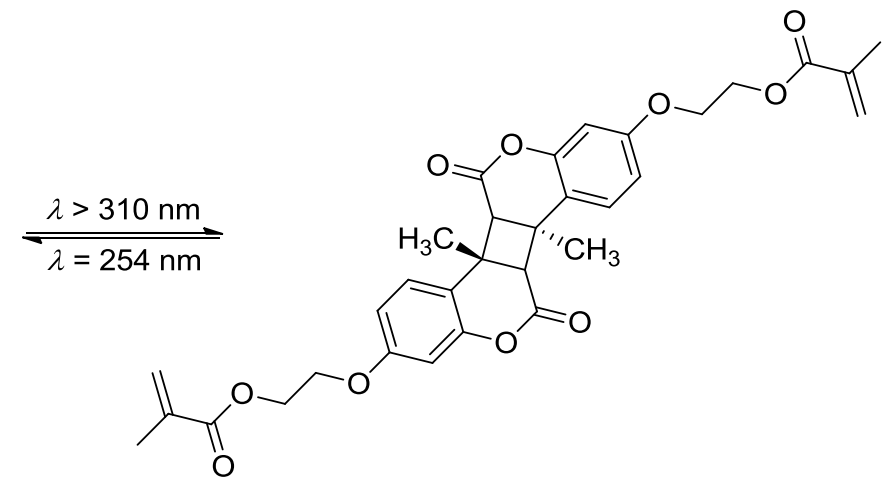

Schema 2-6 Reversible photochemische Dimerisierung des Monomers 7-(2-Methacryloyloxyethoxy)-4-methylcoumarin (CMA).

Bei der Reaktion wird die Bildung von hauptsächlich drei Isomeren beobachtet: syn-Kopf-zu-Kopf, anti-Kopf-zu-Kopf und syn-Kopf-zu-Schwanz (Abbildung 2-14).127 Lewis und Barancyk berichteten, dass bei hohen Konzentrationen die elektronisch angeregten Moleküle im favorisierten Singulettzustand sind und synKopf-zu-Kopf-Isomere entstehen. ${ }^{149}$ Eine geringe Konzentration ermöglicht es den angeregten Molekülen durch ein intersystem-crossing aus dem Singulett- in den Triplettzustand überzugehen, wodurch es vorwiegend zur Bildung von anti-Kopfzu-Kopf-Dimeren kommt. ${ }^{148,149}$ Die Polarität des Lösungsmittels und der Zusatz von Triplettsensibilisatoren (z.B. Benzophenon) haben einen Einfluss auf die Bildung der Isomere. Polare Lösungsmittel favorisieren den Singlettzustand und somit das syn-Produkt, wobei unpolare Lösungsmittel sowie die Zugabe des Sensibilisators zu anti-Produkten führen. ${ }^{152}$

Die photochemische Spaltung des Cyclobutans findet bei UV-Bestrahlung mit der Wellenlänge $\lambda=254 \mathrm{~nm}$ statt und wurde erstmals von Schenk et al. berichtet.147,153 Insgesamt wurde die Dimerspaltung wesentlich weniger intensiv untersucht als die Dimerisierung. ${ }^{127}$ Hasegawa et al. lieferten wichtige Erkenntnisse zur Dimerspaltung des Cyclobutans. ${ }^{154}$ Sie stellten fest, dass die Substituenten am Cyclobutan entscheidenden Einfluss auf die Spaltungsreaktion haben. Sind die Substituenten Teil eines stabilen fünf- oder sechs-gliedrigen Rings, erfolgt eine symmetrische Ringöffnung des Cyclobutans. In Dimeren ohne stabilisierende 
Ringsysteme, wie z.B. Di-ortho-Cumarsäuren, kann auch eine asymmetrische Ringspaltung erfolgen. ${ }^{154}$

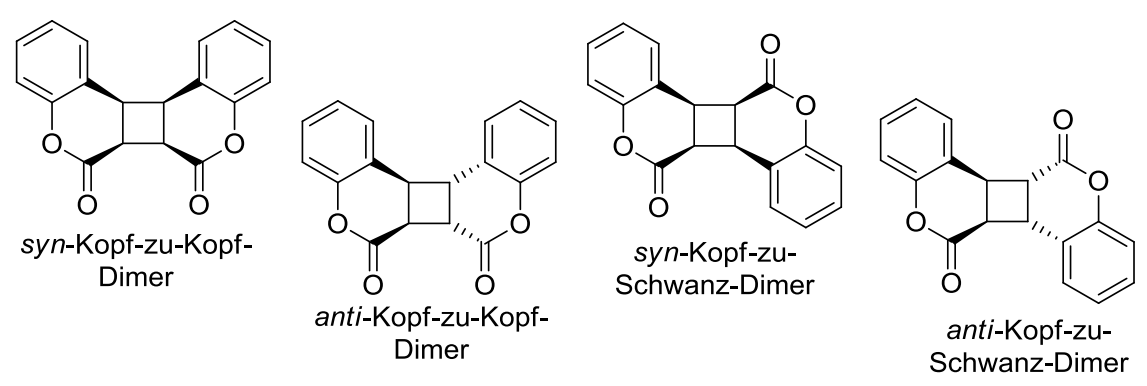

Abbildung 2-14 Darstellung der vier Isomeren der photochemischen Dimerisierung des Coumarins. ${ }^{127}$

Chen et al. untersuchten das Copolymerisationsverhalten von 7-(4-Methylcoumarin)-Acrylat mit unterschiedlichen Acrylaten, Acrylamiden sowie Vinylacetat und die Kinetik der reversiblen Photodimerisierung . 153,155-157 Die photochemische Cycloaddition dünner Polymerfilme erfolgte bei einer Wellenlänge von $\lambda>300 \mathrm{~nm}$ und die Dimerspaltung bei $\lambda=254 \mathrm{~nm}$. Es konnte gezeigt werden, dass beide Reaktionen einer Kinetik erster Ordnung folgen. Mit zunehmendem Anteil der UVaktiven Einheiten stieg der Geschwindigkeitskoeffizient der Vernetzungsreaktion, wobei die Konstante der Dimerspaltung mit zunehmendem Anteil abnahm. ${ }^{156}$ Die Kinetik und die Reaktionsgeschwindigkeiten werden durch die Löslichkeit der Polymere, die Molmasse und die Polymerzusammensetzung beeinflusst. Weiterhin konnte gezeigt werden, dass bei der Dimerspaltung durch die hohe Energie der UV-Strahlung bei $\lambda=254 \mathrm{~nm}$ Nebenreaktionen stattfinden können, die die erneute Photodimerisierung beeinflussen können. Die Copolymerisationsparameter für das System 7-(4-Methylcoumarin)-Acrylat $\left(r_{1}\right)$ und Methylmethacrylat $\left(r_{2}\right)$ wurden durch zwei unterschiedliche Methoden bestimmt und liegen bei $r_{1}=0,45$ und $r_{2}=1,68$. Weitere Untersuchungen zum Copolymerisationsverhalten von unterschiedlichen Coumarin-Derivaten mit verschiedenen Monomeren wie Acrylamiden, Vinylacetat und Acrylaten wurden von Chen et al. durchgeführt. ${ }^{153,155-157}$

Jiang et al. copolymerisierten mit Hilfe des ATRP-Prozesses Methylmethacrylat (MMA) mit CMA und synthetisierten amphiphile Mizellen, wo das Copolymer zur Stabilisierung der Mizellen eingebaut wurde, um vielseitig anwendbare Nanocontainer herzustellen. ${ }^{158}$ Die Reversibilität der photochemischen Reaktionen wurde an Polymerlösungen untersucht, wobei eine vollständige Dimerisierung und 
Spaltung über mindestens fünf Cyclen festgestellt werden konnte. Weiterhin publizierten Sinkel et al. die Verwendung des Coumarin-Derivats CMA in einer radikalischen Copolymerisation mit MMA, um Polymer-Depots für Arzneimittel herzustellen. ${ }^{159}$

Huyck et al. untersuchten das photochemische Verhalten von verschiedenen Copolymeren, bestehend aus einem Coumarin-Derivat und verschiedenen (Meth)Acrylaten. ${ }^{160}$ Sie zeigten, dass die Effizienz der Photodimerisierung bei Polymerfilmen stark von der Kettenbeweglichkeit der Polymere und somit den CoumarinEinheiten abhängt. Die beschränkte Kettenbeweglichkeit der Coumarin-Gruppen im Polymer, das eine Glasübergangstemperatur $T_{\mathrm{g}}$ oberhalb der Bestrahlungstemperatur besitzt, begrenzte den Verbrauch auf $<20 \%$. Die Polymere mit der $T_{\mathrm{g}}$ unterhalb der Bestrahlungstemperatur erreichten bei der Photoreaktion einen Verbrauch von 47-59\%. 


\section{Motivation}

Heutzutage werden immer höhere Anforderungen an die Kunststoffe gestellt und stetig werden neue Anwendungsgebiete wie z. B. in der Nanotechnologie erschlossen. Für die Herstellung neuartiger Materialien sind genaue Kenntnisse der Struktur-Eigenschaftsbeziehungen sowie Polymerisationsverfahren notwendig, die eine exakte Kontrolle der molekularen Architektur eines Polymers ermöglichen. Die RAFT-Polymerisation ist eine sehr vielseitige sowie experimentell vergleichbar unaufwändige Polymerisationstechnik. ${ }^{27,34}$ Sie erlaubt die Synthese von speziell für die entsprechende Verwendung maßgeschneiderten Polymeren, wie z. B. Blockcopolymere.

Blockcopolymere haben in den letzten Jahren besonderes Interesse in Forschung und Technik hervorgerufen. Die Untersuchung der selbstanordnenden Nanostrukturierung in dünnen Filmen durch Mikrophasenseparation stellt ein besonders anregendes Forschungsgebiet dar. So repräsentieren Blockcopolymerfilme eine gute Ergänzung zur bestehenden Fotolithographie-Technik in der Halbleiterindustrie. ${ }^{14}$ Aufgrund der morphologischen Besonderheiten, die das Ergebnis von Mikrophasenseparation sind, können Blockcopolymere zudem einzigartige Eigenschaften zeigen.

Vor diesem Hintergrund wurden Blockcopolymere mit eingebauten, mittels UV-Licht vernetzbaren Einheiten synthetisiert. Die Vernetzungen können auf der einen Seite die Morphologie stabilisieren und zusätzlich zu einer Veränderung der Materialeigenschaften führen. Auf der anderen Seite können die Vernetzungen die Mikrophasenseparation im gleichen Maße verhindern wie z. B. kovalente Bindungen die makroskopische Phasenseparation in Blockcopolymeren unterbinden. Von besonderem Interesse ist die Untersuchung der Konkurrenz zwischen Selbstorganisation in Blockcopolymeren und den topologischen Einschränkungen durch Vernetzungen, da dieses bis heute ein wenig erforschtes Gebiet ist.

Die Anordnung eines Blockcopolymersystems kann beispielsweise durch die Temperatur oder die Vernetzungsdichte beeinflusst werden, wobei letztere durch die Anzahl der eingebauten UV-vernetzbaren Einheiten variiert werden kann. Der Einbau der Vernetzer kann in beiden Blöcken oder nur in einem Block erfolgen. Durch die Vernetzung des Systems kann der jeweilige Präparationszustand eingefroren werden. Wird beispielsweise der geordnete Zustand des Systems 
durch die Vernetzung fixiert und das synthetisierte Netzwerk durch Veränderung der Temperatur aus dem Gleichgewichtszustand gedrängt, ist eine Relaxation nur eingeschränkt möglich und der bei hohen Temperaturen bevorzugte ungeordnete Zustand des Systems kann nicht ausgebildet werden. Das System liegt somit in einem Nichtgleichgewichtszustand vor. Aufgrund der topologischen Einschränkungen kann sich nur ein geringer Anteil des Systems entmischen. Das anschließende Öffnen der Vernetzungen ermöglicht die Untersuchung des Übergangs vom geordneten in den ungeordneten Zustand. Dies ist insbesondere aus Sicht der Grundlagenforschung von Bedeutung, da hier besonders Interesse an der Struktur und Dynamik von Nichtgleichgewichtszuständen besteht.

Das Ziel der vorliegenden Arbeit war es, einheitliche phasenseparierende Blockcopolymere mit eingebauten UV-vernetzbaren Einheiten mittels RAFTPolymerisation zu synthetisieren und deren besondere topologische sowie mechanische Eigenschaften zu analysieren. Vom besonderen Interesse bei den eingebauten Einheiten war die Möglichkeit einer reversiblen Photodimerisierung. Weiterhin wurde der Einfluss der topologischen Einschränkungen durch Vernetzung auf die Selbstorganisation in Blockcopolymeren untersucht. 


\section{Auswertung und Diskussion}

\subsection{Copolymerisation der Coumarin-Derivate}

In diesem Abschnitt werden die Ergebnisse der Copolymerisationen von Methylmethacrylat (MMA) und $n$-Butylacrylat (BA) mit photochemisch-vernetzbaren Monomeren präsentiert. Abbildung 4-1 zeigt die in diesem Abschnitt verwendeten Monomere. In der vorliegenden Arbeit wurden verschiedene Coumarin-Derivate als vernetzungsfähige Gruppen in die Polymere eingebaut. Die Coumarin-Derivate bestehen aus einer UV-aktiven Einheit und einer Monomereinheit, an der die RAFT-Polymerisation stattfindet. 7-Acryloyloxyethoxy4-methylcoumarin (CA) und 7-(2-Methacryloyloxythoxy)-4-methylcoumarin (CMA) wurden in einer Zwei-Schritt-Synthese hergestellt, die in Abschnitt 6.6.1 näher beschrieben wird. Bei den in dieser Arbeit verwendeten CoumarinDerivaten wird die reaktive Doppelbindung für die Polymerisation und die UVaktive Einheit durch eine Brücke, bestehend aus zwei Kohlenstoffatomen, voneinander getrennt, wodurch eine erfolgreiche Copolymerisation angenommen wird. Für einen<smiles>C=C(C)C(=O)OCCOc1ccc2c(C)cc(=O)oc2c1</smiles>

7-(2-Methacryloyloxyethoxy)-4methylcoumarin (CMA)<smiles>C=CC(=O)OCCOc1ccc2c(C)cc(=O)oc2c1</smiles>

7-Acryloyloxyethoxy-4methylcoumarin (CA)<smiles>C=C(C)C(=O)OC</smiles>

Methylmethacrylat (MMA)<smiles>C=CC(=O)OCCCC</smiles>

n-Butylacrylat

(BA)

Abbildung 4-1 Coumarin-Derivate CMA und CA, sowie Monomere Methylmethacrylat und $n$-Butylacrylat.

statistischen Einbau der vernetzungsfähigen Gruppen in das Copolymer sollten die Monomer-Grundgerüste so ähnlich wie möglich sein, sodass große Unähnlichkeiten in ihrem Polymerisationsverhalten untereinander vermieden werden (siehe Abschnitt 2.2).

Abbildung 4-2 zeigt das in dieser Arbeit verwendete RAFT-Agens, das sich durch besonders gute Kontrolle bei Polymerisationen von Methacrylaten, Methacryl-<smiles>CCCCCCCCCCCCCCCCC(=O)O</smiles>

Abbildung 4-2 RAFT-Agens 4-Cyano-4[(dodecylsulfanylthiocarbonyl)sulfanyl]Pentansäure. 
amiden und Styrol auszeichnet. Das Trithiocarbonat ist weiterhin für Polymerisationen von Acrylaten geeignet und ist kommerziell erhältlich.

Um den erfolgreichen Einbau der vernetzungsfähigen Gruppen nachzuweisen und den Gehalt des Comonomers im Polymer $\mathrm{zu}$ bestimmen, wurden die synthetisierten Copolymere mittels NMR-Spektroskopie untersucht. Die Untersuchung des Prozesses der photochemischen Vernetzung des Comonomers im Polymer wurde quantitativ mit der UV-Vis-Spektroskopie untersucht und wird in Abschnitt 4.7 näher erläutert.

\subsubsection{Copolymerisation von MMA mit CMA}

Die Copolymerisation wurde als Lösungspolymerisation bei $70{ }^{\circ} \mathrm{C}$ durchgeführt, da CMA eine relativ schlechte Löslichkeit im Comonomer MMA zeigte. Als Lösungsmittel wurde DMF verwendet. Das Polymerisationsverhalten der Comonomere wurde mit der Homopolymerisation von MMA bei gleichen Reaktionsbedingungen verglichen. Um den Einfluss des Comonomers auf die Polymerisation zu betrachten, wurde der Anteil von CMA variiert. Zwei unterschiedliche Polymerisationsmischungen mit jeweils 1 und 5 mol\% CMA wurden vorbereitet. Die Zusammensetzungen der Polymerisationsmischungen sind in Tabelle 4-1 aufgeführt (siehe Anhang A, Tabelle A-1 bis Tabelle A-3).

Tabelle 4-1 Übersicht über die Zusammensetzungen der Polymerisationsmischungen für die Homopolymerisation von MMA und die Copolymerisationen von MMA mit 1 mol\% CMA sowie $5 \mathrm{~mol}^{\prime} \% \mathrm{CMA}$. Die Polymerisationen wurden in DMF bei $70^{\circ} \mathrm{C}$ durchgeführt.

\begin{tabular}{|l|l|l|l|l|}
\hline Polymerisation & Molverhältnis & $\begin{array}{l}\boldsymbol{c}_{\text {RAFT }} \\
\text { [mmol/L] }\end{array}$ & $\begin{array}{l}\boldsymbol{c}_{\text {AIBN }} \\
{[\mathbf{m m o l} / \mathbf{L}]}\end{array}$ & $\begin{array}{l}\boldsymbol{f}_{\text {CMA }} \\
{[\mathbf{m o l} \%]}\end{array}$ \\
\hline Homopolymerisation A & MMA:RAFT:AIBN & 24 & 5 & $/$ \\
\hline Copolymerisation A & $=$ & 24 & 5 & 1 \\
\hline Copolymerisation B & $200: 1: 0,2$ & 23 & 5 & 5 \\
\hline
\end{tabular}

Abbildung 4-3 zeigt die ermittelten GP-Chromatogramme für die Homopolymerisation A sowie die jeweiligen Copolymerisationen (A und B) von MMA und CMA. Die Polymere wurden gegen einen linearen PMMA-Standard kalibriert. Zur Erstellung der GP-Chromatogramme wurde der RI-Detektor verwendet (siehe Abbildung 4-3). Die Chromatogramme der Homo- und Copolymerisationen zeigen enge, monomodale Molmassenverteilungen (MMV). Das kontrollierte Reaktionsverhalten während der Polymerisationen wird durch die Zunahme der Molmassen mit steigendem Monomerumsatz verdeutlicht. 

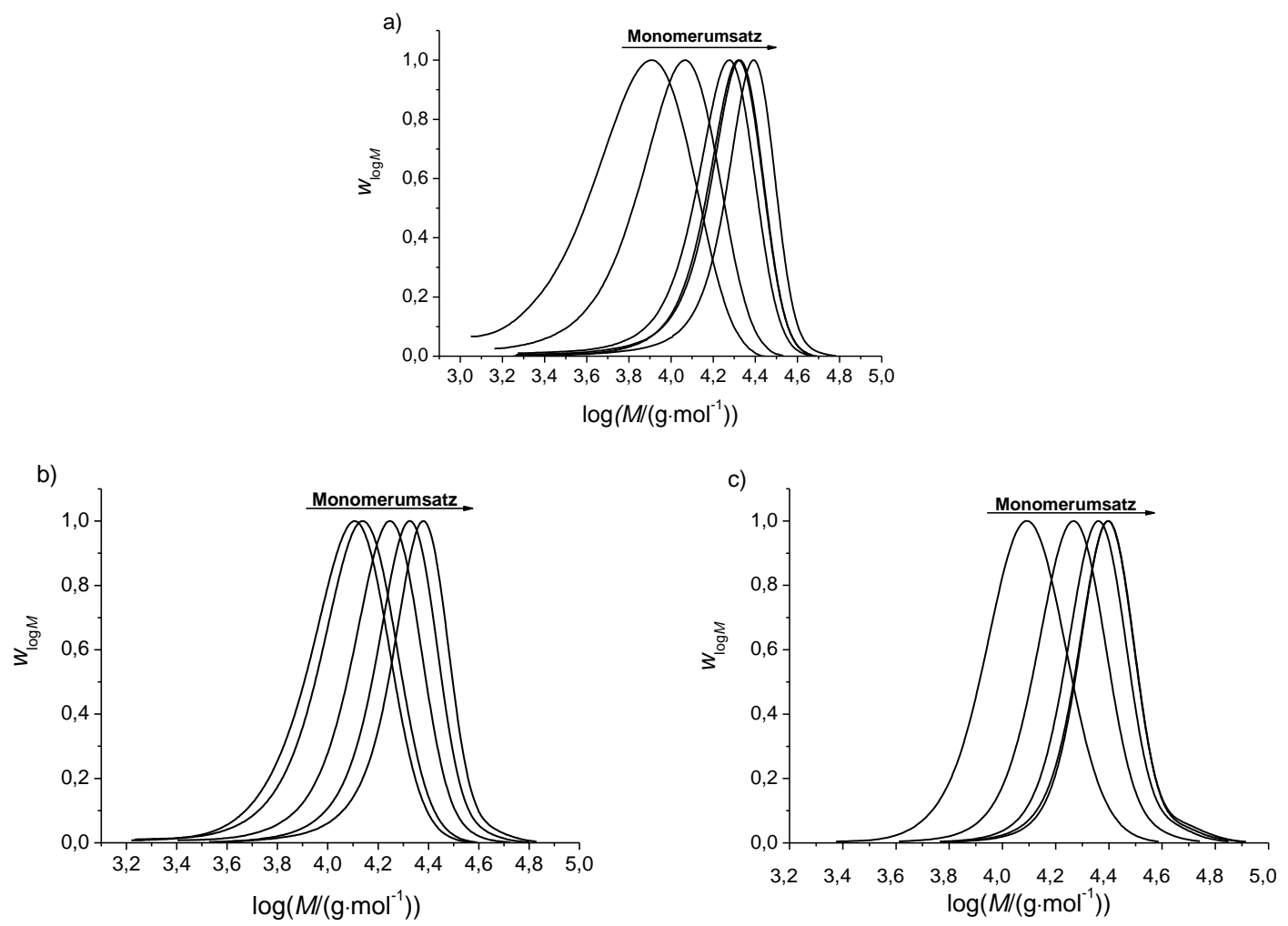

Abbildung 4-3 GP-Chromatogramme (RI-Signal): a) Homopolymerisation A, b) Copolymerisation B mit 1 mol\% CMA, c) Copolymerisation A mit 5 mol\% CMA.

Die Entwicklung der Molmasse in Abhängigkeit des Monomerumsatzes für die Homopolymerisation A und die beiden Copolymerisationen A und B wird in Abbildung 4-4 dargestellt. Das obere Diagramm in Abbildung 4-4 zeigt die Entwicklung der Dispersität bei entsprechendem Monomerumsatz für die unterschiedlichen Polymerisationen. 


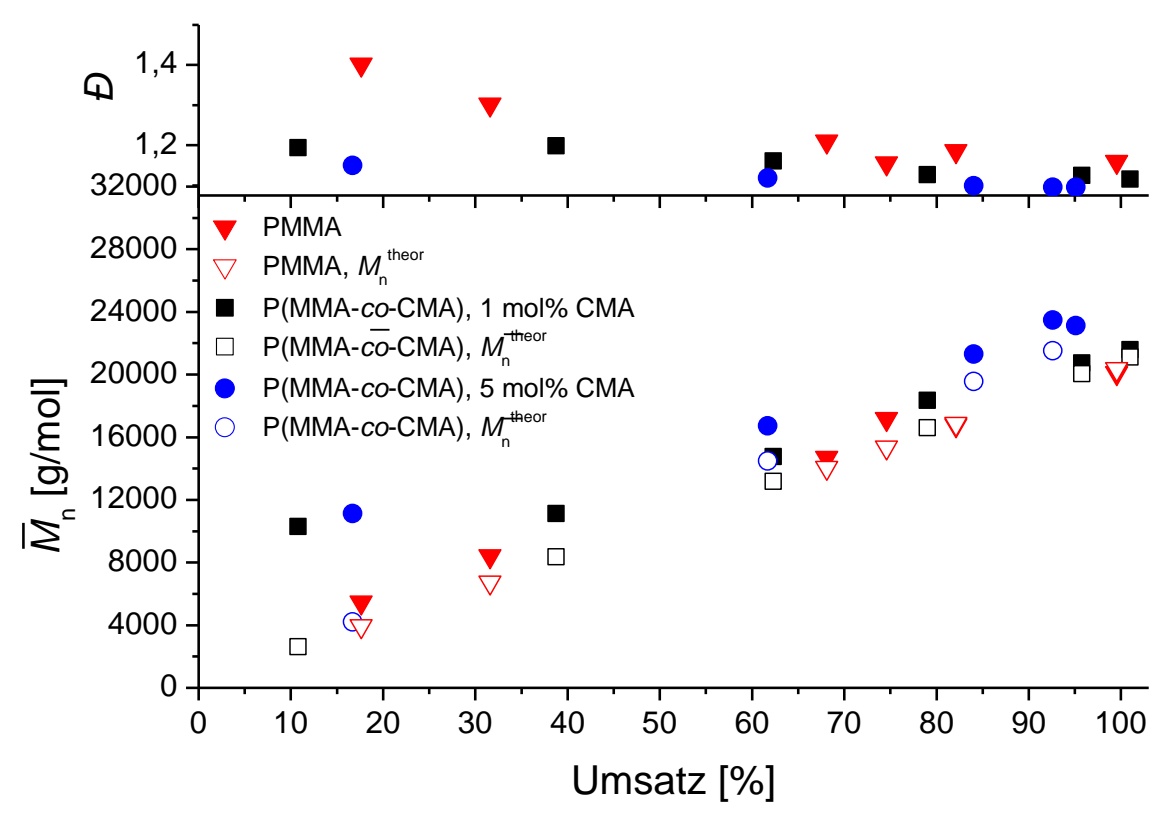

Abbildung 4-4 Entwicklung der Molmasse und der Dispersität in Abhängigkeit vom Monomerumsatz für die Homopolymerisation A (rot), die Copolymerisation A mit 5 mol\% CMA (blau) und die Copolymerisation B mit 1 mol\% CMA (schwarz).

Abbildung 4-4 zeigt einen linearen Anstieg der Molmassen mit zunehmendem Monomerumsatz. Dies deutet auf ein kontrolliertes Verhalten der Polymerisation hin, das in Abschnitt 2.3.1 erklärt wird. Die gemessenen Molmassen sind in guter Übereinstimmung mit den theoretisch berechneten Werten. Die ermittelten Dispersitäten nehmen mit zunehmendem Monomerumsatz ab. Für alle Polymerisationsbedingungen liegen die Dispersitäten ab einem Umsatz von $32 \%$ im Bereich von 1,1 bis 1,2, was dem charakteristischen Wert einer kontrollierten RAFT-Polymerisation entspricht (siehe Abschnitt 2.4). Weiterhin kann geschlussfolgert werden, dass durch die Zugabe des Comonomers CMA der kontrollierte Charakter der Reaktion verbessert wird. Im Vergleich zum Homopolymer steigen die mit der GPC ermittelten Molmassen mit zunehmendem CMA-Gehalt, was auf einen erfolgreichen Einbau des Comonomers schließen lässt.

Allerdings ist festzuhalten, dass beide Copolymerisationen hohe Molmassen bei niedrigen Monomerumsätzen aufweisen. Diese starke Abweichung spricht gegen ein kontrolliertes Verhalten im Anfangsbereich der Polymerisation. Sowohl die Molmassenauftragung als auch die Dispersitäten zeigen, dass sich das RAFTGleichgewicht erst nach einer gewissen Anfangszeit einstellt. Dieses Verhalten wird auch Hybridverhalten genannt, das in Abschnitt 2.3.1 erklärt wird. Weiterhin ist zu erwähnen, dass für das Copolymer-System kein geeigneter Standard 
vorhanden ist. Für die Bestimmung der Molmassen wird ein PMMA-Standard zur Kalibrierung verwendet, wodurch die erhaltenen Werte für die Molmassen nur als Näherungswerte zu betrachten sind.

Zusammenfassend konnte festgestellt werden, dass die Copolymerisation von MMA und CMA mittels RAFT-Polymerisation erfolgreich durchgeführt werden kann. Die Reaktion findet in einem angemessenen Zeitintervall statt und kann somit als geeigneter Weg für den Einbau UV-aktiver Vernetzer in das Polymer genutzt werden. Polymere mit einer eng verteilten MMV $(\fallingdotseq<1,4)$ und Molmassen bis maximal $23000 \mathrm{~g} / \mathrm{mol}$ konnten synthetisiert werden. Im folgenden Unterabschnitt sollen die Zusammensetzungen der Copolymere bestimmt werden.

\section{Bestimmung der UV-aktiven Einheiten im Comonomer P(MMA-co-CMA)}

Die Bestimmung der Zusammensetzung des statistischen Copolymers ist ein wichtiger Aspekt dieser Arbeit. Das endgültige Blockcopolymer soll aus zwei Blöcken bestehen, wobei der erste Block aus zwei statistisch verteilten Monomeren besteht und der zweite Block aus einem Monomer. Mit Hilfe der NMRSpektroskopie kann unter anderem der Einbau des UV-aktiven Monomers in das Copolymer nachgewiesen und die Zusammensetzung des Copolymers bestimmt werden.

Eine in der Literatur bekannte Methode für die Bestimmung der Zusammensetzung ist der Vergleich spezifischer NMR-Signale für die unterschiedlichen Monomere, welche letztendlich das Copolymer bilden. ${ }^{159}$ Abbildung 4-5 zeigt ein Beispiel für ein ${ }^{1} \mathrm{H}-\mathrm{NMR}$-Spektrum für das Copolymer P(MMA-co-CMA). ${ }^{1} \mathrm{H}-\mathrm{NMR}$ Spektren wurden für alle Proben der Copolymerisation A und B aufgenommen und analysiert.

Das ${ }^{1} \mathrm{H}-\mathrm{NMR}$-Spektrum in Abbildung 4-5 zeigt alle erwarteten Signale für das Copolymer P(MMA-co-CMA). Für ein besseres Verständnis sollen die unterschiedlichen Signale im Folgenden erklärt werden. Im tiefen Feldbereich sind die Peaks der aromatischen Protonen des CMAs $\mathrm{C}^{3}-\mathrm{H}, \mathrm{C}^{4}-\mathrm{H}$ und $\mathrm{C}^{5}-\mathrm{H}$, sowie das olefinische Proton $\mathrm{C}^{7}-\mathrm{H}$ zu finden. Der Bereich zwischen 7,6 und 6 ppm stellt die charakteristischen Peaks des UV-aktiven Monomers dar. Die Protonen der Methacrylateinheit des CMAs erschienen vor dem Einbau in das Copolymer im Bereich von 5,7 ppm und 6,03 ppm und sind nach der Copolymerisation nicht mehr sichtbar, was für einen erfolgreichen Einbau des CMAs in das Copolymer hindeutet. Die Protonen der C2-Alkylbrücke $\left(\mathrm{C}^{1}-\mathrm{H}\right.$ und $\left.\mathrm{C}^{2}-\mathrm{H}\right)$ sind im Bereich zwischen 4,2 und 4,3 ppm zu finden. Zusätzlich ist die $\mathrm{CH}_{3}-\mathrm{Gruppe}\left(\mathrm{C}^{6}-\mathrm{H}\right)$ des UV- 
aktiven Monomers im Bereich von 2,4 ppm zu finden und liegt somit gut separiert vom Polymerrückgrat vor. Der dominierende Peak bei 3,6 ppm stellt den charakteristischen Peak der $\mathrm{CH}_{3}$-Gruppe des MMA-Monomers dar. Das Polymerrückgrat ist im Bereich von 0,8 bis 2 ppm zu finden und zeigt die charakteristischen Peaks der beiden Monomere, die im Polymerrückgrat eingebaut sind.

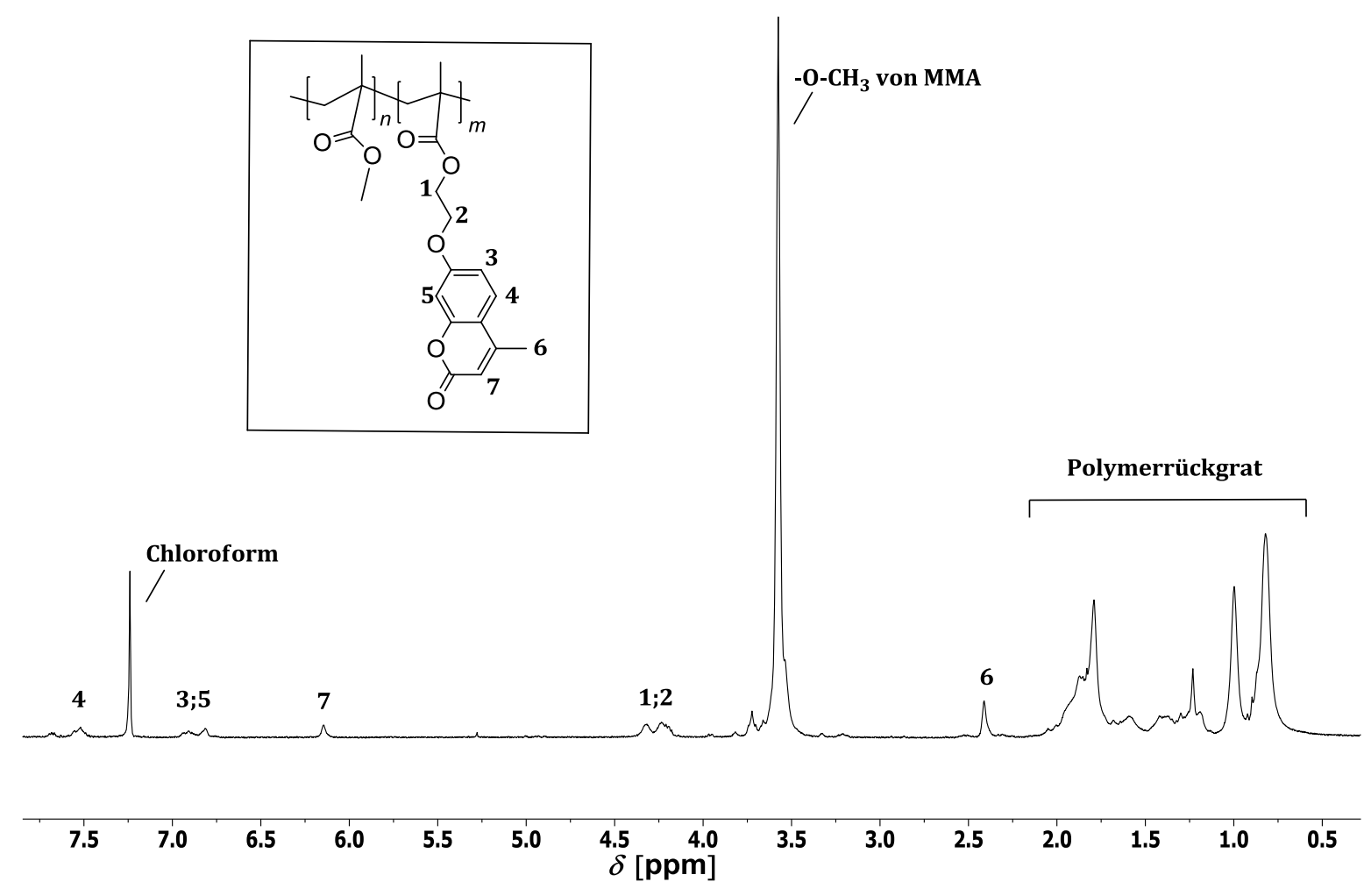

Abbildung 4-5 ${ }^{1} \mathrm{H}-\mathrm{NMR}$-Spektrum von P(MMA-co-CMA) aufgenommen in $\mathrm{CDCl}_{3}$.

Im Folgenden sollen die Ergebnisse der spektroskopischen Untersuchungen gezeigt werden. Abbildung 4-6 zeigt die übereinander gelegten ${ }^{1} \mathrm{H}-\mathrm{NMR}-\mathrm{Spektren}$ der Copolymerisation A mit 5 mol\% CMA. 


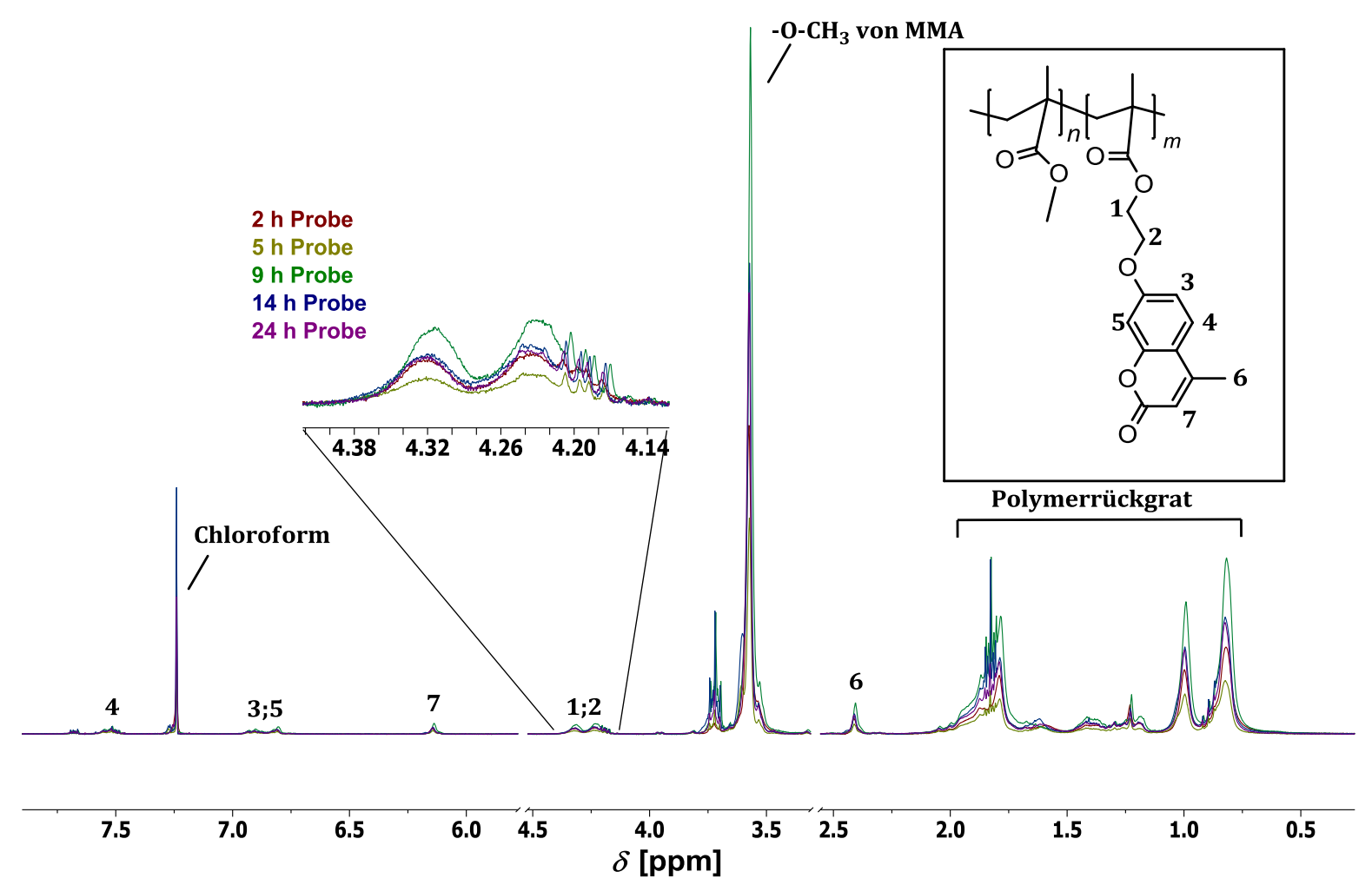

Abbildung 4-6 Übereinandergelegte 1H-NMR-Spektren für alle Proben der Copolymerisation A. Die Spektren wurden in $\mathrm{CDCl} 3$ gemessen.

Die Zusammensetzung des Copolymers wurde durch Integration der Signale der C2-Alkylbrücke bei 4,3 - 4,2 ppm (Bildausschnitt Abbildung 4-6) und des Signals bei 3,6 ppm der $\mathrm{CH}_{3}$-Gruppe des MMAs bestimmt. Dazu müssen die Integrale ( $I_{\mathrm{MMA}}$, $\left.I_{\mathrm{CMA}}\right)$ auf ihre jeweilige Protonenanzahl bezogen werden. Gleichung (25) und (26) stellen das beispielhaft dar:

$$
\begin{gathered}
I_{\mathrm{MMA}}=1 \equiv 3 \text { Protonen } \\
\frac{I_{\mathrm{MMA}}}{3 \text { Protonen }}=\frac{1}{3}=0,3333 \\
I_{\mathrm{CMA}}=0,11 \equiv 4 \text { Protonen } \\
\frac{I_{\mathrm{CMA}}}{4 \text { Protonen }}=\frac{0,11}{4}=0,0275
\end{gathered}
$$

Für die Berechnung des molaren Anteils $F$ des jeweiligen Monomers im Polymer werden die oben berechneten Werte in Relation zueinander gesetzt. 


$$
\begin{gathered}
F_{\mathrm{MMA}}=\frac{\frac{I_{\mathrm{MMA}}}{3}}{\frac{I_{\mathrm{MMA}}}{3}+\frac{I_{\mathrm{CMA}}}{4}}=\frac{0,33}{0,36}=0,924=92,4 \mathrm{~mol} \% \\
F_{\mathrm{CMA}}=\frac{\frac{I_{\mathrm{CMA}}}{4}}{\frac{I_{\mathrm{MMA}}}{3}+\frac{I_{\mathrm{CMA}}}{4}}=\frac{0,0275}{0,36}=0,076=7,6 \mathrm{~mol} \%
\end{gathered}
$$

Es erfolgte eine analoge Auswertung für die ${ }^{1} \mathrm{H}-\mathrm{NMR}$-Spektren der Copolymerisation B. Die Ergebnisse beider Copolymerisationen sind in Tabelle 4-2 zusammengefasst.

Tabelle 4-2 Auflistung der Monomereingabe von CMA, $f_{\mathrm{CMA}}$, und des molaren Anteils von CMA im Polymer, $F_{\mathrm{CMA}}$, für die Copolymerisationen A und B.

\begin{tabular}{|l|l|l|}
\hline Polymer & $\boldsymbol{f}_{\mathrm{CMA}}[\mathrm{mol} \%]$ & $\boldsymbol{F}_{\mathrm{CMA}}[\mathrm{mol} \%]$ \\
\hline $\begin{array}{l}\text { P(MMA-co-CMA), } \\
\text { Copolymerisation A }\end{array}$ & 5 & 8 \\
\hline $\begin{array}{l}\text { P(MMA-co-CMA), } \\
\text { Copolymerisation B }\end{array}$ & 1 & 4 \\
\hline
\end{tabular}

Alle NMR-Spektren wurden mit einer verzögerten Relaxationszeit ( $d=15 \mathrm{~s})$ aufgenommen, da die Kerne im Polymer wesentlich längere Relaxationszeiten benötigen als kleinere Moleküle. Tabelle 4-2 zeigt, dass der berechnete molare Anteil des CMAs $\left(F_{\mathrm{CMA}}\right)$ im Copolymer höher ist als die Monomereingabe $\left(f_{\mathrm{CMA}}\right)$.

Die molaren Anteile der einzelnen Monomere wurden mit den Molmassen der Monomere $\left(M_{\mathrm{MMA}}=100,12 \mathrm{~g} / \mathrm{mol}, M_{\mathrm{CMA}}=288,30 \mathrm{~g} / \mathrm{mol}\right)$ in deren Gewichtsanteile umgerechnet. In den folgenden Gleichungen soll das beispielhaft dargestellt werden:

$$
\begin{gathered}
F_{\mathrm{MMA}} \cdot M_{\mathrm{MMA}}=m_{\mathrm{MMA}}=0,924 \mathrm{~mol} \% \cdot 100,12 \frac{\mathrm{g}}{\mathrm{mol}}=92,51 \mathrm{~g} \\
F_{\mathrm{CMA}} \cdot M_{\mathrm{CMA}}=m_{\mathrm{CMA}}=0,076 \mathrm{~mol} \% \cdot 288,3 \frac{\mathrm{g}}{\mathrm{mol}}=21,91 \mathrm{~g} \\
m_{\mathrm{MMA}}+m_{\mathrm{CMA}}=m_{\text {gesamt }}=92,51 \mathrm{~g}+21,91 \mathrm{~g}=114,42 \mathrm{~g} \\
\equiv 100 \text { Gew. } \%
\end{gathered}
$$


Somit ergibt sich:

$$
\begin{aligned}
& m_{\mathrm{MMA}} \equiv 80,85 \text { Gew. } \% \\
& m_{\mathrm{CMA}} \equiv 19,15 \text { Gew. } \%
\end{aligned}
$$

Auf die mit der GPC ermittelte Molmasse des Polymers (RI-Detektor) wurde die Avogadro-Konstante angewandt, um das Gewicht eines Polymer-Moleküls $\left(w_{\text {Polymer }}\right) \mathrm{zu}$ erhalten. Der CMA- und MMA-Anteil im Polymer wird mit den jeweiligen Gewichtsanteilen (Gew.\%сма, Gew.\%ммА) ermittelt. Dieser Wert wird im Anschluss durch das Gewicht eines einzelnen Monomer-Moleküls dividiert, um die Anzahl an jeweiligen Monomereinheiten im Polymer zu bestimmen. Im Folgenden wird die Berechnung der CMA- und MMA-Einheiten beispielhaft gezeigt.

$$
\begin{gathered}
w_{\text {Polymer }}=\frac{\bar{M}_{\mathrm{n}}}{N_{\mathrm{A}}}=\frac{16700 \mathrm{~g} / \mathrm{mol}}{6,022 \cdot 10^{23} \mathrm{~mol}^{-1}}=2,78 \cdot 10^{-20} \mathrm{~g} \equiv 100 \mathrm{Gew} . \% \\
\text { CMA: } 19,15 \mathrm{Gew} . \% \equiv 5,32 \cdot 10^{-21} \mathrm{~g} \\
\text { MMA: } 80,85 \mathrm{Gew} . \% \equiv 2,24 \cdot 10^{-20} \mathrm{~g} \\
w_{\mathrm{CMA}}=\frac{M_{\mathrm{CMA}}}{N_{\mathrm{A}}}=\frac{288,3 \mathrm{~g} / \mathrm{mol}}{6,022 \cdot 10^{23} \mathrm{~mol}^{-1}}=4,79 \cdot 10^{-22} \mathrm{~g} \\
w_{\mathrm{MMA}}=\frac{M_{\mathrm{MMA}}}{N_{\mathrm{A}}}=\frac{100,12 \mathrm{~g} / \mathrm{mol}^{2}}{6,022 \cdot 10^{23} \mathrm{~mol}-1}=1,66 \cdot 10^{-22} \mathrm{~g} \\
\text { Anzahl der CMA }- \text { Einheiten }=\frac{5,32 \cdot 10^{-21} \mathrm{~g}}{4,79 \cdot 10^{-22} \mathrm{~g}} \approx 11 \\
\text { Anzahl der MMA }- \text { Einheiten }=\frac{2,24 \cdot 10^{-20} \mathrm{~g}}{1,66 \cdot 10^{-22} \mathrm{~g}} \approx 135
\end{gathered}
$$

Bei diesem Beispiel konnten 135 MMA-Einheiten und 11 CMA-Einheiten berechnet werden. Tabelle 4-3 fast die Ergebnisse für die Copolymerisation von MMA und 5 mol\% CMA zusammen. 
Tabelle 4-3 Bestimmung der CMA- und MMA-Einheiten in verschiedenen Proben der Copolymerisation A.

\begin{tabular}{|l|l|l|l|l|l|l|l|}
\hline $\begin{array}{l}\text { Zeit } \\
{[\mathbf{h}]}\end{array}$ & $\begin{array}{l}\overline{\boldsymbol{M}}_{\mathbf{n}} \\
\mathbf{[ g} / \mathbf{m o l}]\end{array}$ & $\begin{array}{l}\text { Umsatz } \\
{[\mathbf{6}]}\end{array}$ & $\begin{array}{l}\boldsymbol{F}_{\text {CMA }} \\
{[\mathbf{m o l} \% \mathbf{l}}\end{array}$ & $\begin{array}{l}\boldsymbol{F}_{\text {MMA }} \\
{[\mathbf{m o l} \% \mathbf{]}}\end{array}$ & $\begin{array}{l}\text { Anzahl } \\
\text { der CMA- } \\
\text { Einheiten }\end{array}$ & $\begin{array}{l}\text { Anzahl } \\
\text { der MMA- } \\
\text { Einheiten }\end{array}$ & $\begin{array}{l}\text { CMA/MMA- } \\
\text { Einheiten } \\
\text { im Polymer }\end{array}$ \\
\hline $\mathbf{2}$ & 11100 & 17 & 8 & 92 & 8 & 88 & 0,09 \\
\hline $\mathbf{5}$ & 16700 & 62 & 8 & 92 & 11 & 135 & 0,08 \\
\hline $\mathbf{9}$ & 21300 & 84 & 8 & 92 & 14 & 172 & 0,08 \\
\hline $\mathbf{1 5}$ & 23500 & 93 & 8 & 92 & 16 & 190 & 0,08 \\
\hline $\mathbf{2 4}$ & 23100 & 95 & 8 & 92 & 15 & 187 & 0,08 \\
\hline
\end{tabular}

Es konnte für alle Proben der Copolymerisation A ein höherer CMA-Einbau $\left(F_{\mathrm{CMA}} \approx 8 \mathrm{~mol} \%\right)$ im Polymer festgestellt werden. Diese abweichende Berechnung kann zum Teil an der Ungenauigkeit der Integration der Signale in den NMRSpektren liegen. Bei einer Vergrößerung des Integrationsbereiches von 0,05 ppm zu beiden Seiten des Signals beträgt die Abweichung vom oben genannten molaren Anteil ( $\left.F_{\mathrm{CMA}}\right)$ ca. \pm 2 mol\%. Zudem kann die unzureichende Molmassenbestimmung der Polymere bei den GPC-Messungen zu abweichenden molaren Anteilen führen. Um eine genaue Aussage zur Reaktivität der einzelnen Monomere treffen zu können, müssten die Copolymerisationsparameter bestimmt werden. Die Bestimmung der Parameter war für diese Arbeit nicht zwingend notwendig und kann in zukünftigen Arbeiten noch durchgeführt werden.

Bei Betrachtung der Verhältnisse der eingebauten Monomere im Polymer (siehe Tabelle 4-3) sind für alle Proben der Copolymerisation von MMA mit CMA keine großen Abweichungen zu erkennen. Die molaren Anteile $F_{\mathrm{CMA}}$ und $F_{\mathrm{MMA}}$ sind während des gesamten Reaktionsverlaufes konstant. Es wird angenommen, dass ein statistisches Copolymer entsteht. Der Bildausschnitt in Abbildung 4-6 zeigt die charakteristischen Signale des Monomers CMA und auch hier ist mit zunehmendem Reaktionsverlauf keine Tendenz der Signale zu sehen.

Weiterhin wurde der Verlauf für die Copolymerisation B mittels NMRSpektroskopie analysiert. Die in $\mathrm{CDCl}_{3}$ aufgenommenen NMR-Spektren ähneln den Spektren in Abbildung 4-6 und werden aus diesem Grund hier nicht gezeigt. Auch für diese Copolymerisation wurde die Zusammensetzung des Copolymers mittels der oben beschriebenen Methode bestimmt. Tabelle 4-4 fasst die erhaltenen Ergebnisse zusammen. 
Tabelle 4-4 Bestimmung der CMA- und MMA-Einheiten in verschiedenen Proben der Copolymerisation B.

\begin{tabular}{|l|l|l|l|l|l|l|l|}
\hline $\begin{array}{l}\text { Zeit } \\
{[\mathbf{h}]}\end{array}$ & $\begin{array}{l}\overline{\boldsymbol{M}}_{\mathbf{n}} \\
{[\mathbf{g} / \mathbf{m o l}]}\end{array}$ & $\begin{array}{l}\text { Umsatz } \\
{[\mathbf{6}]}\end{array}$ & $\begin{array}{l}\boldsymbol{F}_{\text {CMA }} \\
{[\mathbf{m o l} \mathbf{m}]}\end{array}$ & $\begin{array}{l}\boldsymbol{F}_{\text {MMA }} \\
{[\mathbf{m o l} \% \mathbf{]}}\end{array}$ & $\begin{array}{l}\text { Anzahl } \\
\text { der CMA- } \\
\text { Einheiten }\end{array}$ & $\begin{array}{l}\text { Anzahl } \\
\text { der MMA- } \\
\text { Einheiten }\end{array}$ & $\begin{array}{l}\text { CMA/MMA- } \\
\text { Einheiten } \\
\text { im Polymer }\end{array}$ \\
\hline $\mathbf{1 , 5}$ & 10300 & 11 & 4 & 96 & 3 & 93 & 0,04 \\
\hline $\mathbf{3}$ & 11100 & 39 & 4 & 96 & 4 & 98 & 0,04 \\
\hline $\mathbf{5}$ & 14800 & 62 & 4 & 96 & 6 & 131 & 0,04 \\
\hline $\mathbf{8}$ & 18400 & 79 & 4 & 96 & 7 & 163 & 0,04 \\
\hline $\mathbf{1 4}$ & 21500 & 96 & 4 & 96 & 7 & 194 & 0,04 \\
\hline $\mathbf{2 4}$ & 20600 & 100 & 4 & 96 & 8 & 182 & 0,04 \\
\hline
\end{tabular}

Bei den molaren Anteilen $F_{\mathrm{CMA}}$ und $F_{\mathrm{MMA}}$ sind während des gesamten Reaktionsverlaufes keine Abweichungen zu erkennen. Mit zunehmendem Monomerumsatz bleibt das Verhältnis der eingebauten Monomere im Polymer für alle Proben der Copolymerisation gleich groß, sodass die Bildung eines statistischen Copolymers angenommen wird. In den Copolymerisationen A und B ist der molare Anteil des CMAs $F_{\text {CMA }}$ höher als die Monomereingabe $f_{\text {CMA }}$. Vor dem Hintergrund der Literatur kann für beide Polymerisationen ein statistisches Copolymer angenommen werden. ${ }^{159,161}$

\subsubsection{Homopolymerisation von CMA}

Eine weitere Überlegung war, dass der Einbau der UV-aktiven Gruppen nicht nur durch ein statistisches Copolymer stattfinden muss, sondern dass ein kompletter Block aus UV-Vernetzern bestehen könnte. Somit wurde eine RAFT-Polymerisation von CMA getestet. In der Literatur konnte gezeigt werden, dass eine Herstellung von Blockcopolymeren bestehend aus Polyethylenglycol und CMA mittels einer ATRP-Polymerisation möglich ist, wobei CMA meistens sowohl unter kontrollierten als auch unter konventionellen radikalischen Polymerisationsbedingungen in Copolymerisationen mit anderen Monomeren verwendet wird. ${ }^{162}$

Die Zusammensetzung der Polymerisationsmischung für die Homopolymerisation von CMA ist in Tabelle 4-5 aufgeführt (siehe Anhang A, Tabelle A-4). Die Chromatogramme für die Homopolymersation von CMA in Abbildung 4-7 zeigen das fehlende Wachsen der Molmassen mit zunehmendem Monomerumsatz. 
Tabelle 4-5 Übersicht über die Zusammensetzungen der Polymerisationsmischungen für die Homopolymerisation von CMA bei $70{ }^{\circ} \mathrm{C}$ in DMF. Das verwendete RAFT-Agens ist in Abbildung 4-2 dargestellt.

\begin{tabular}{|l|l|l|l|l|}
\hline Polymerisation & Molverhältnis & $\begin{array}{l}\boldsymbol{C}_{\mathrm{CMA}} \\
{[\mathbf{m m o l} / \mathbf{L}]}\end{array}$ & $\begin{array}{l}\boldsymbol{C}_{\mathrm{RAFT}} \\
{[\mathbf{m m o l} / \mathbf{L}]}\end{array}$ & $\begin{array}{l}\boldsymbol{c}_{\mathrm{AIBN}} \\
{[\mathbf{m m o l} / \mathbf{L}]}\end{array}$ \\
\hline Homopolymerisation B & $\begin{array}{c}\text { CMA:RAFT:AIBN } \\
=\end{array}$ & 200 & 10 & 2 \\
\hline & $20: 1: 0,2$ & & & \\
\hline
\end{tabular}

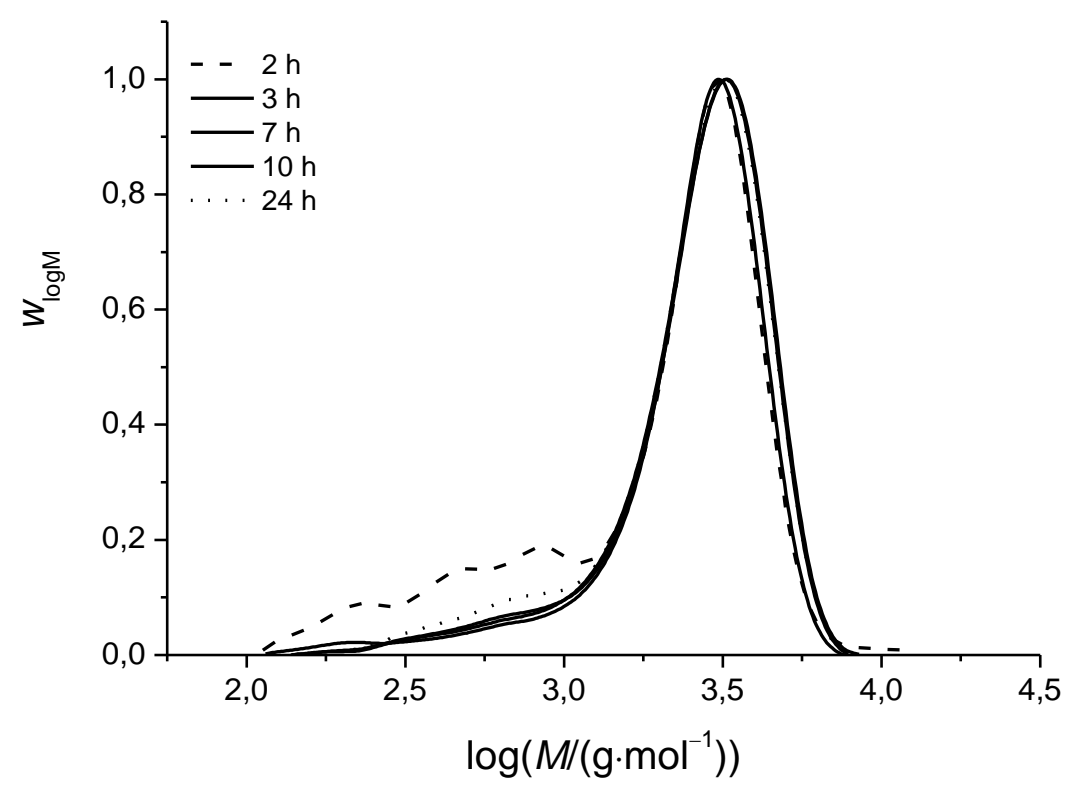

Abbildung 4-7 GP-Chromatogramm für die Homopolymerisation B.

Die Entwicklung der Molmasse und der Dispersität gegen den Monomerumsatz ist in Abbildung 4-8 dargestellt. Bei der Auftragung der Molmasse gegen den Monomerumsatz ist kein linearer Verlauf zu erkennen, woraus man auf ein nicht kontrolliertes Polymerisationsverhalten schließen kann. Weiterhin wurden für alle Proben der Homopolymerisation Dispersitäten von $Ð>1,4$ erhalten. 


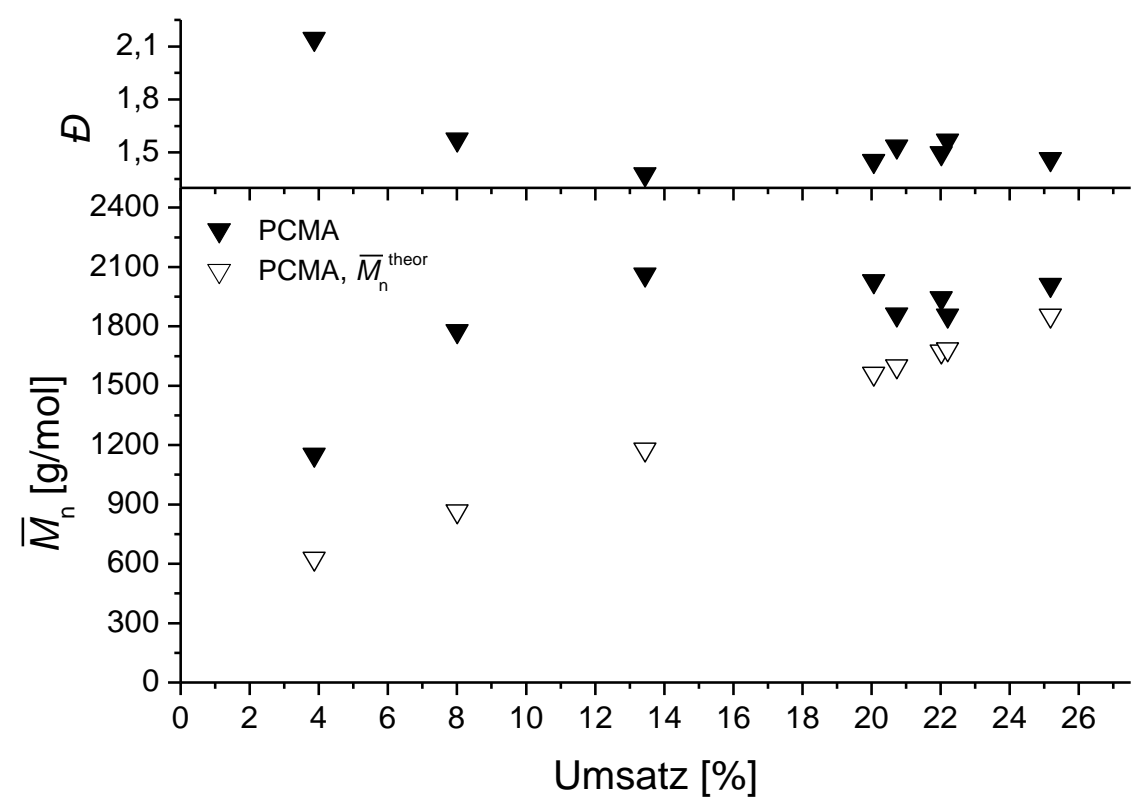

Abbildung 4-8 Entwicklung der Molmasse und der Dispersität in Abhängigkeit vom Monomerumsatz für die Homopolymerisation B.

Abbildung 4-8 zeigt deutlich einen Polymerisationsstart, jedoch erreicht die Molmasse bei einem Monomerumsatz von $13 \%$ ein Plateau. Es wird eine Molmasse von ca. $2000 \mathrm{~g} / \mathrm{mol}$ bei einem Monomerumsatz von $13 \%$ erreicht, wobei bis $\mathrm{zu}$ einem Umsatz von $25 \%$ keine großen Molmassenänderungen festgestellt werden können.

Zusammenfassend kann gesagt werden, dass die RAFT-Polymersition von CMA nicht zufriedenstellend durchgeführt werden konnte. Das System zeigt eine fehlende Kontrolle beim Polymerisationsverhalten. Somit kann der Einbau der UVaktiven Gruppen nur über ein statistisches Copolymer erfolgen.

\subsubsection{Copolymerisation von BA mit CA}

Zusätzlich zu MMA, wurde eine Copolymerisation von BA mit einem CoumarinDerivat durchgeführt. In einer vorangegangenen Arbeit im Arbeitskreis Vana wurde bereits die Copolymerisation von BA mit CMA durchgeführt, wobei ein verzögerter Polymerisationsstart (Induktion) festgestellt wurde und die Polymerisation insgesamt ein nicht gut kontrollierbares Verhalten zeigte. ${ }^{163}$ Da sich Acrylate und Methacrylate in ihrem Polymerisationsverhalten stark voneinander unterscheiden, wurde für die folgende Copolymerisation von BA die Acrylatvariante des Coumarin-Derivats (CA) verwendet. 
Die Copolymerisation wurde als Lösungspolymerisation bei $70^{\circ} \mathrm{C}$ durchgeführt, da CA eine relativ schlechte Löslichkeit im Comonomer BA zeigte. Als Lösungsmittel wurde DMF verwendet. Das Polymerisationsverhalten der Comonomere wurde mit der Homopolymerisation von BA bei gleichen Reaktionsbedingungen verglichen. Die Zusammensetzungen der Homopolymerisation von BA und die Copolymerisation von BA mit 5 mol\% CA sind in Tabelle 4-6 aufgeführt (siehe auch Tabelle A-5 und Tabelle A-6, Anhang A). Abbildung 4-9 zeigt die Chromatogramme für die Homopolymerisation $C$ und die Copolymerisation C. Zur Erstellung der Chromatogramme wurde eine BA-Kalibrierung verwendet.

Tabelle 4-6 Übersicht über die Zusammensetzungen der Polymerisationsmischungen für die Homopolymerisation von BA und die Copolymerisationen von BA mit 5 mol\% CA. Die Polymerisationen wurden in DMF bei $70^{\circ} \mathrm{C}$ durchgeführt.

\begin{tabular}{|l|c|l|l|l|}
\hline Polymerisation & Molverhältnis & $\begin{array}{l}\boldsymbol{c}_{\text {RAFT }} \\
{[\mathbf{m m o l} / \mathbf{L}]}\end{array}$ & $\begin{array}{l}\boldsymbol{c}_{\text {AIBN }} \\
{[\mathbf{m m o l} / \mathbf{L}]}\end{array}$ & $\boldsymbol{f}_{\mathrm{CMA}}[\mathbf{m o l} \% \mathbf{}$ \\
\hline Homopolymerisation C & BA:RAFT:AIBN & 13 & 3 & - \\
\hline Copolymerisation C & $=$ & 19 & 4 & 5 \\
\hline & $200: 1: 0,2$ & & & \\
\hline
\end{tabular}
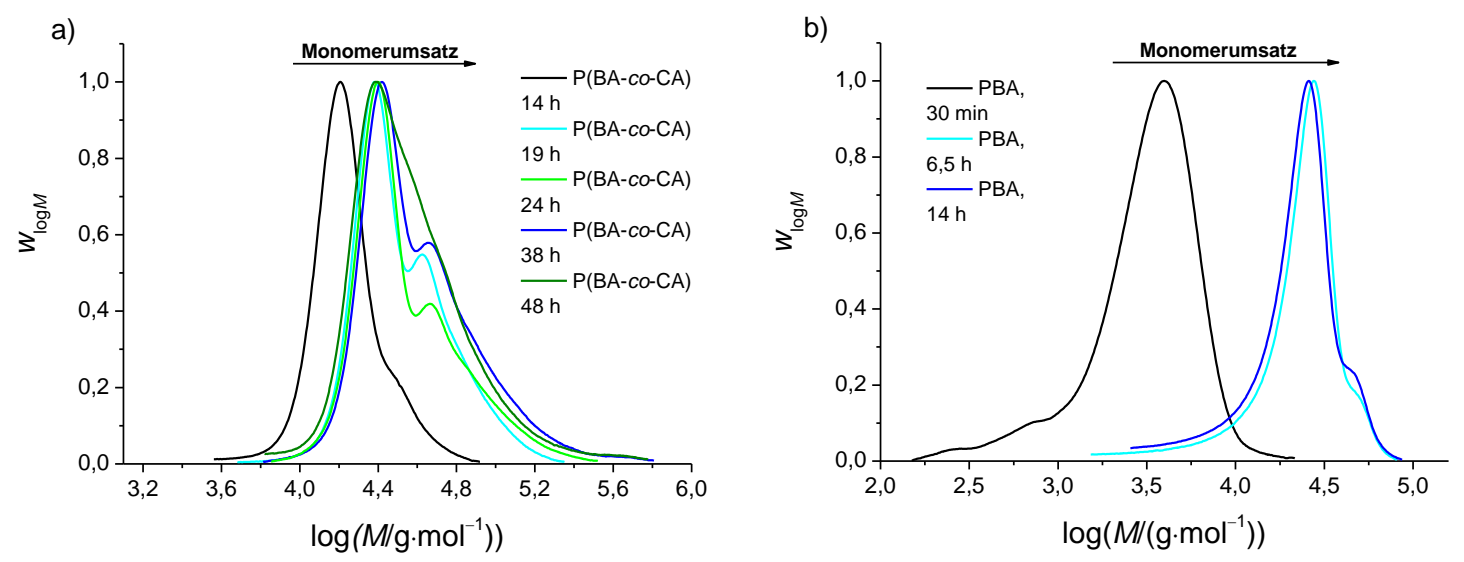

Abbildung 4-9 GP-Chromatogramme (RI-Signal): a) Copolymerisation C, b) Homopolymerisation C.

Die Homo- und Copolymerisation zeigen im Bereich höherer Molmassen eine Schulterbildung, was im Fall der Homopolymerisation von BA ein bekanntes Phänomen ist und auf eine Kettenverzweigung (engl.: chain branching) oder Terminierung während der Polymerisation zurückzuführen ist. ${ }^{16}$ Durch den bei der Copolymerisation abrupten Anstieg der Molmasse und Dispersität kann darauf geschlossen werden, dass die Reaktion insgesamt nicht gut kontrolliert verläuft. 
Die Entwicklung der Molmasse in Abhängigkeit des Monomerumsatzes für die beiden Polymerisationen ist in Abbildung 4-10 dargestellt. Das obere Diagramm in Abbildung 4-10 zeigt die Entwicklung der Dispersität bei entsprechendem Monomerumsatz für die Polymerisationen.

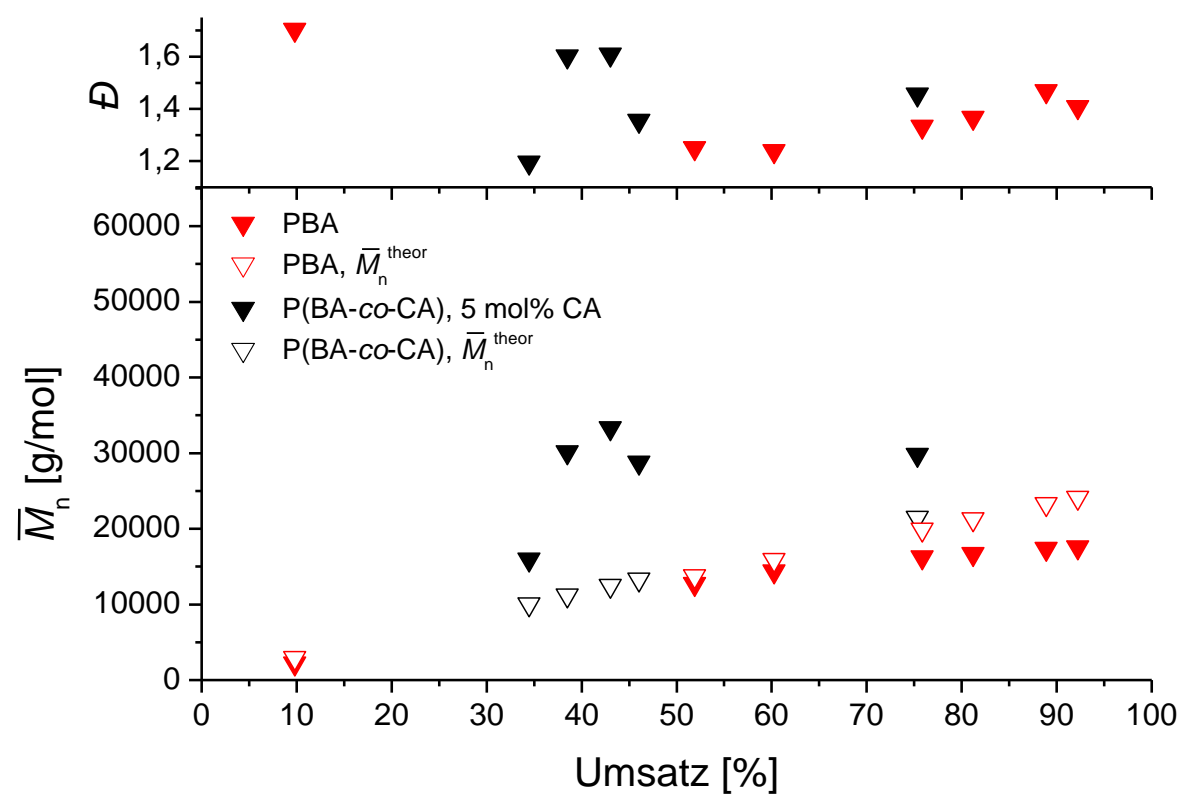

Abbildung 4-10 Entwicklung der Molmasse und der Dispersität in Abhängigkeit vom Monomerumsatz für die Homopolymerisation C (rot) und der Copolymerisation C (schwarz).

Bei den Proben der Copolymerisation ist kein linearer Anstieg der Molmassen mit zunehmendem Monomerumsatz zu erkennen. Die Reaktionszeit der gesamten Polymerisation erstreckte sich von 3 bis 48 h. Erst nach einer Reaktionszeit von $14 \mathrm{~h}$ konnte ein Monomerumsatz von $35 \%$ festgestellt werden, wohingegen bei der Homopolymerisation nach ähnlichem Zeitintervall ein Umsatz von $X>90 \%$ erreicht wurde. Insgesamt scheint die Zugabe von CA den Polymerisationsstart zu verzögern.

Zusätzlich wurde hier die Zusammensetzung der Copolymere nach der in Abschnitt 4.1.1 beschriebenen Methode bestimmt. Abbildung 4-11 zeigt ein Beispiel für ein ${ }^{1} \mathrm{H}-\mathrm{NMR}$-Spektrum für das Copolymer $\mathrm{P}(\mathrm{BA}-\mathrm{Co}-\mathrm{CA}) .{ }^{1} \mathrm{H}-\mathrm{NMR}-$ Spektren wurden für alle Proben der Copolymerisation $\mathrm{C}$ aufgenommen und analysiert. 


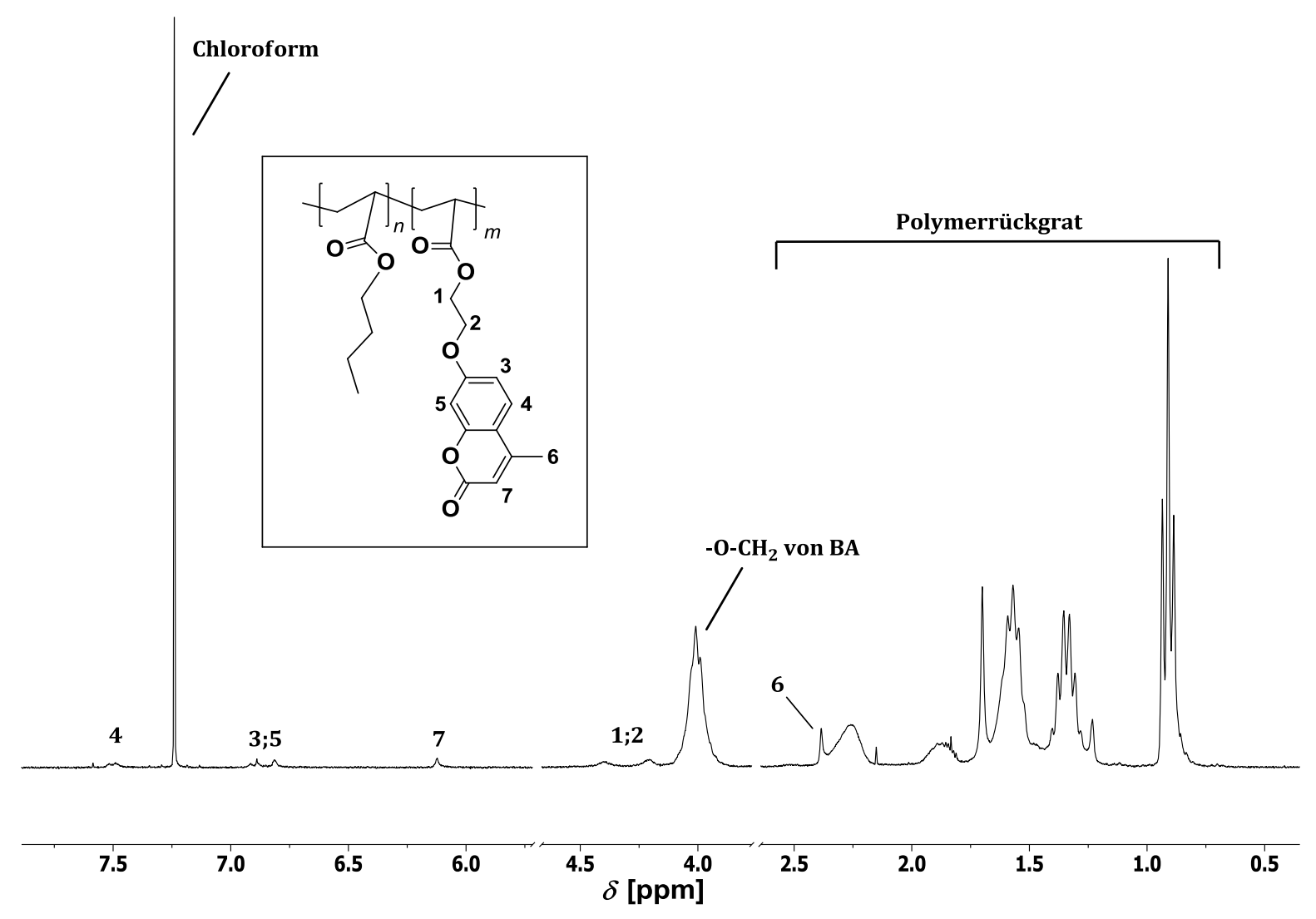

Abbildung 4-11 ${ }^{1} \mathrm{H}-\mathrm{NMR}-S p e k t r u m$ von $\mathrm{P}(\mathrm{BA}-\mathrm{co}-\mathrm{CA})$ aufgenommen in $\mathrm{CDCl}_{3}$.

Im tiefen Feldbereich sind die Peaks der aromatischen Protonen des CAs $\mathrm{C}^{3}-\mathrm{H}, \mathrm{C}^{4}-\mathrm{H}$ und $\mathrm{C}^{5} \mathrm{H}$, sowie das olefinische Proton $\mathrm{C}^{7}-\mathrm{H}$ zu finden. Der Bereich zwischen 7,5 und 6,1 ppm stellt die charakteristischen Peaks des UV-aktiven Monomers dar. Die Protonen der Acrylateinheit des CAs erschienen vor dem Einbau in das Copolymer im Bereich von 6 bis 6,4 ppm und sind nach der Copolymerisation nicht mehr sichtbar, was für einen erfolgreichen Einbau des CAs in das Copolymer hindeutet. Die Protonen der C2-Alkylbrücke ( $\mathrm{C}^{1}-\mathrm{H}$ und $\left.\mathrm{C}^{2}-\mathrm{H}\right)$ sind im Bereich von 4,2 bis 4,4 ppm zu finden. Der dominierende Peak bei 4,01 ppm stellt den charakteristischen Peak der -O- $\mathrm{CH}_{2}$-Gruppe des BA-Monomers dar. Das Polymerrückgrat erstreckt sich im Bereich von 0,9 bis 2,4 ppm und zeigt die charakteristischen Peaks der Monomereinheiten. Weiterhin ist bei 2,39 ppm das Signal der $\mathrm{CH}_{3}$-Gruppe ( $\left.\mathrm{C}^{6}-\mathrm{H}\right)$ des $\mathrm{CAs}$ zu erkennen.

Die Zusammensetzung des Copolymers wurde durch Integration der Signale der C2-Alkylbrücke bei 4,2 bis 4,4 ppm und des Signals bei 4,01 ppm der -0- $\mathrm{CH}_{2}$ Gruppe des BAs bestimmt. Tabelle 4-7 fasst die Ergebnisse für die Copolymerisation von BA und CA zusammen. 
Tabelle 4-7 Bestimmung der CA- und BA-Einheiten in verschiedenen Proben der Copolymerisation $\mathrm{C}$.

\begin{tabular}{|l|l|l|l|}
\hline Zeit [h] & $\overline{\boldsymbol{M}}_{\mathbf{n}}[\mathbf{g} / \mathbf{m o l}]$ & $\boldsymbol{F}_{\mathrm{CA}}[\mathbf{m o l} \mathbf{0}]$ & $\boldsymbol{F}_{\mathrm{BA}}[\mathbf{m o l} \mathbf{\%}]$ \\
\hline $\mathbf{1 4}$ & 15900 & 9 & 91 \\
\hline $\mathbf{1 9}$ & 28800 & 9 & 91 \\
\hline $\mathbf{2 4}$ & 29800 & 9 & 91 \\
\hline $\mathbf{3 8}$ & 33300 & 9 & 91 \\
\hline $\mathbf{4 8}$ & 30100 & 8 & 92 \\
\hline
\end{tabular}

Die molaren Anteile $F_{\mathrm{CA}}$ und $F_{\mathrm{BA}}$ sind während des gesamten Reaktionsverlaufes konstant. Somit wird angenommen, dass ein statistisches Copolymer entsteht und beide Monomere im Verlauf der Copolymerisation gleichmäßig eingebaut werden.

Trotz alledem konnte eine erfolgreiche RAFT-Polymerisation von BA mit CA nicht durchgeführt werden, da eine zu geringe Polymerisationskontrolle und zu lange Reaktionszeiten festgestellt wurden. Somit wird der Einbau des UV-aktiven Monomers im Blockcopolymer über den MMA-Block erfolgen. 


\subsection{4 Übersicht verwendeter makroRAFT-Agenzien}

Die Copolymerisation von MMA und CMA zeigte insgesamt eine gute Polymerisationskontrolle, annehmbare Reaktionszeiten und einen erfolgreichen Einbau der Coumarin-Gruppen. Für die Synthese verschiedener Blockcopolymere soll das Copolymer-System P(MMA-co-CMA) als makroRAFT-Agens (makromolekulares RAFT-Agens) dienen. Für eine bessere Übersicht in den folgenden Abschnitten werden die verwendeten makroRAFT-Agenzien in Tabelle 4-8 zusammengefasst. Für die Herstellung der für die Blockcopolymerisationen verwendeten makroRAFT-Agenzien wurden jeweils unterschiedliche MonomerRAFT-Verhältnisse verwendet (siehe Anhang A, Tabelle A-8).

Tabelle 4-8 Übersicht über Monomereingabe von CMA, $f_{\mathrm{CMA}}$, molaren Anteil von CMA im Copolymer, $F_{\mathrm{CMA}}$, Molmasse $\bar{M}_{\mathrm{n}}$, Dispersität $Ð$ von den makroRAFT-Agenzien P(MMA-co-CMA) und Anzahl der CMA- und MMA-Einheiten im jeweiligen Copolymer.

\begin{tabular}{|c|c|c|c|c|c|c|}
\hline Probe & $\begin{array}{l}f_{\text {CMA }} \\
{[\mathrm{mol} \%]}\end{array}$ & $\begin{array}{l}F_{\text {CMA }} \\
{[\mathrm{mol} \%]}\end{array}$ & $\begin{array}{l}\bar{M}_{\mathrm{n}} \\
\text { [g/mol] }\end{array}$ & $\boldsymbol{D}$ & $\begin{array}{l}\text { Anzahl } \\
\text { der CMA- } \\
\text { Einheiten }\end{array}$ & $\begin{array}{l}\text { Anzahl } \\
\text { der MMA- } \\
\text { Einheiten }\end{array}$ \\
\hline $\begin{array}{l}\text { I } \\
\text { [P(MMA- } \\
\text { co-CMA)] }\end{array}$ & 5 & 8 & 12000 & 1,20 & 9 & 95 \\
\hline $\begin{array}{l}\text { II } \\
{[\mathrm{P}(\mathrm{MMA}-} \\
\text { co-CMA })]\end{array}$ & 5 & 7 & 14300 & 1,19 & 9 & 117 \\
\hline $\begin{array}{l}\text { III } \\
\text { [P(MMA- } \\
\text { co-CMA)] }\end{array}$ & 5 & 7 & 5800 & 1,18 & 4 & 48 \\
\hline $\begin{array}{l}\text { IV } \\
\text { [P(MMA- } \\
\text { co-CMA)] }\end{array}$ & 5 & 7 & 47500 & 1,30 & 29 & 390 \\
\hline
\end{tabular}




\subsection{Blockcopolymerisation}

Der letzte Syntheseschritt beinhaltet die Herstellung von Blockcopolymeren (BCP), die in diesem Abschnitt beschrieben wird. Beide Blöcke sollen sich in ihren Eigenschaften stark voneinander unterscheiden, in der Annahme, dass beide Blöcke somit eine gute Mikrophasenseparation (MPS) eingehen können. Die in dieser Arbeit hergestellten BCP sollen aus harten und weichen Blöcken bestehen. Der erste Block ist ein statistisches Copolymer, das sich aus MMA und dem UVaktiven CMA zusammensetzt. Der zweite Block besteht aus $n$-Butylacrylat (siehe Abbildung 4-12, (BA)), das aufgrund seiner niedrigen Glasübergangstemperatur (unterhalb der Raumtemperatur (RT), $T_{\mathrm{g}}=-40^{\circ} \mathrm{C}$ ) ein weiches Material bildet. 164 Im Gegensatz dazu besitzt MMA eine $T_{\mathrm{g}}$ weit oberhalb der RT $\left(105^{\circ} \mathrm{C}\right)$ und ist somit ein hartes Material. ${ }^{164}$ Die Herstellung des statistischen Copolymers P(MMAco-CMA) wird in Abschnitt 4.1.1 beschrieben und soll für die zukünftigen Blockcopolymerisationen als makroRAFT-Agens dienen, da der lebende Charakter durch die an den Ketten vorhandenen RAFT-Einheiten erhalten bleibt. Die für die folgenden Blockcopolymerisationen verwendeten makroRAFT-Agenzien sind in Tabelle 4-8 in Abschnitt 4.1.4 zusammengefasst. Es ist zu erwarten, dass sich aufgrund der unterschiedlichen Eigenschaften der Monomere und Polymere im System die Kontrolle der RAFT-Polymerisation verringert.

Da sich die Eigenschaften beider Blöcke auf das Phasenverhalten des Blockcopolymers auswirken, wird ein weiteres BCP-System hergestellt, wo die Eigenschaften beider Blöcke sich weniger stark voneinander unterscheiden. Dafür wurde ein BCP-System ausgesucht, das aus zwei Methacrylaten besteht. Das statistische Copolymer P(MMA-co-CMA) bildet auch hier den ersten Block und der zweite Block besteht aus Butylmethacrylat (siehe Abbildung 4-12, (BMA)). Im Vergleich zu BA besitzt das Monomer BMA eine wesentlich höhere $T_{\mathrm{g}}\left(20^{\circ} \mathrm{C}\right) .{ }^{164}$ Die thermischen Untersuchungen der jeweiligen BCP erfolgt im Abschnitt 4.3.

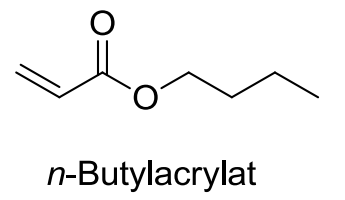

(BA)

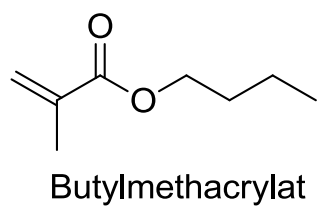

(BMA)

Abbildung 4-12 Monomere $n$-Butylacrylat und Butylmethacrylat.

Es muss erwähnt werden, dass es sich bei den ermittelten Molmassen der BCP um Näherungswerte handelt, da für diese Copolymer-Systeme keine Standards für die Kalibrierung der GPC vorhanden sind. Eine Bestimmung mittels Massenspektrometrie kann nicht durchgeführt werden, da bei dieser Methode das 
Detektionslimit bei $20000 \mathrm{~g} / \mathrm{mol}$ liegt und die hier synthetisierten BCP höhere Molmassen aufweisen.

\subsubsection{Herstellung von $\mathrm{P}(\mathrm{MMA}-\mathrm{co}-\mathrm{CMA})-b$-PBA}

Für die Herstellung des Blockcopolymers P(MMA-co-CMA)- $b$-PBA wurde das statistische Copolymer P(MMA-co-CMA) als makroRAFT-Agens verwendet. In Hinsicht auf die spätere Untersuchung der Blockcopolymere auf Mikrophasenseparation sollen die Polymere nicht nur in ihrer Zusammensetzung variieren, sondern auch in den einzelnen Blocklängen. Für die Variation des Polymerisationsgrades $N$ der BCP wurden makroRAFT-Agenzien verwendet, die sich in ihrer Blocklänge unterscheiden (siehe Tabelle 4-8). Das Lösungsmittel Toluol ist für beide Polymere (PMMA, PBA) geeignet und somit wurde die Reaktion in diesem Lösungsmittel durchgeführt. Die Zusammensetzungen der unterschiedlichen Polymerisationsmischungen ist in Tabelle 4-9 aufgeführt (siehe auch Anhang A, Tabelle A-9 bis Tabelle A-11).

Tabelle 4-9 Übersicht über die Zusammensetzungen der Polymerisationsmischungen für die Blockcopolymerisationen von BA mit verschiedenen makroRAFT-Agenzien (siehe Tabelle 4-8). Die Polymerisationen wurden in Toluol bei $70{ }^{\circ} \mathrm{C}$ durchgeführt.

\begin{tabular}{|l|l|l|l|l|}
\hline Polymerisation & Molverhältnis & $\begin{array}{l}\text { makroRAFT- } \\
\text { Agens }\end{array}$ & $\begin{array}{l}\boldsymbol{c}_{\mathrm{BA}} \\
\text { [mol/L] }\end{array}$ & $\begin{array}{l}\boldsymbol{c}_{\text {AIBN }} \\
{[\mathbf{m m o l} / \mathbf{L}]}\end{array}$ \\
\hline $\begin{array}{l}\text { Blockcopolymerisation } \\
\text { A }\end{array}$ & & $\mathbf{I}$ & 4,36 & 1 \\
\hline $\begin{array}{l}\text { Blockcopolymerisation } \\
\text { B }\end{array}$ & $\begin{array}{c}\text { BA:makroRAFT:AIBN } \\
=\end{array}$ & IV & 1,78 & 1 \\
\hline $\begin{array}{l}\text { Blockcopolymerisation } \\
\text { C }\end{array}$ & $\begin{array}{c}1000: 1: 0,2 \\
\text { III }\end{array}$ & III & 4,36 & 1 \\
\hline
\end{tabular}

Die Molmassenverteilungen der einzelnen Proben der Blockcopolymerisation A sind in Abbildung 4-13 dargestellt. Das rote Chromatogramm zeigt dabei den Ausgangsblock P(MMA-co-CMA) I und die in blau und grün dargestellten Chromatogramme stellen die erhaltenen BCP nach einer Reaktionszeit von 10, 15, 20,25 und 30 min dar. 


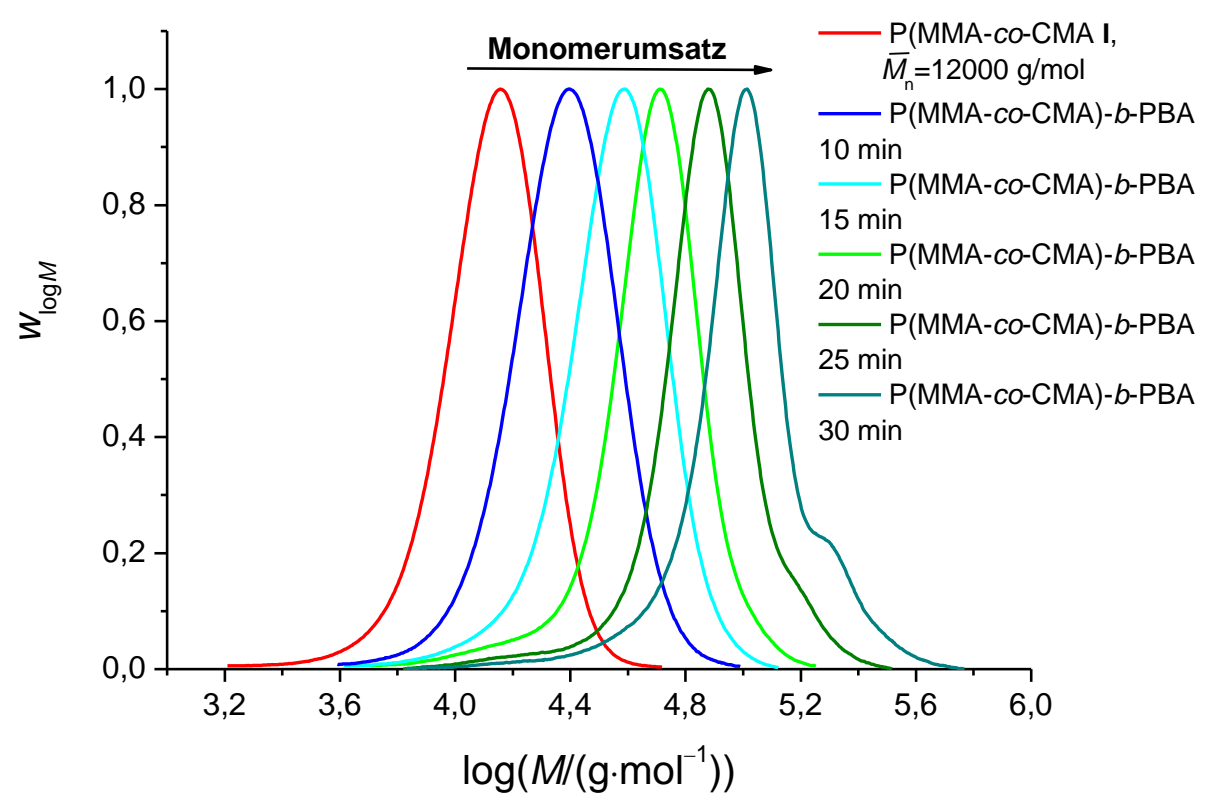

Abbildung 4-13 GP-Chromatogramm (RI-Detektor) von der Blockcopolymersation A, gemessen in THF.

Die engen monomodalen Molmassenverteilungen sowie die Zunahme der Molmasse mit steigendem Monomerumsatz verdeutlichen das kontrollierte Reaktionsverhalten während der Polymerisation. Jedoch zeigen die erhaltenen MMV nach 25 und 30 min eine kleine Schulter im Bereich zu höheren Molmassen, was auf eine Kettenverzweigung oder Terminierung während der Polymerisation hindeutet. Dieses Phänomen konnte schon oft für Acrylat-Polymerisationen beobachtet werden. ${ }^{16}$

Abbildung 4-14 zeigt die Entwicklung der Molmasse und Dispersität in Abhängigkeit des Monomerumsatzes für eine Blockcopolymerisation mit dem Molverhältnis BA:makroRAFT:AIBN = 1000:1:0,2. Die niedrigen Dispersitäten sowie der lineare Verlauf der Molmassen deuten auf ein kontrolliertes Verhalten des Polymerisationsprozesses. Weiterhin sind in Abbildung 4-14 die Molmasse und die Dispersität des Ausgangsmaterials bei einem Monomerumsatz von $0 \%$ dargestellt (blauer Pfeil). 


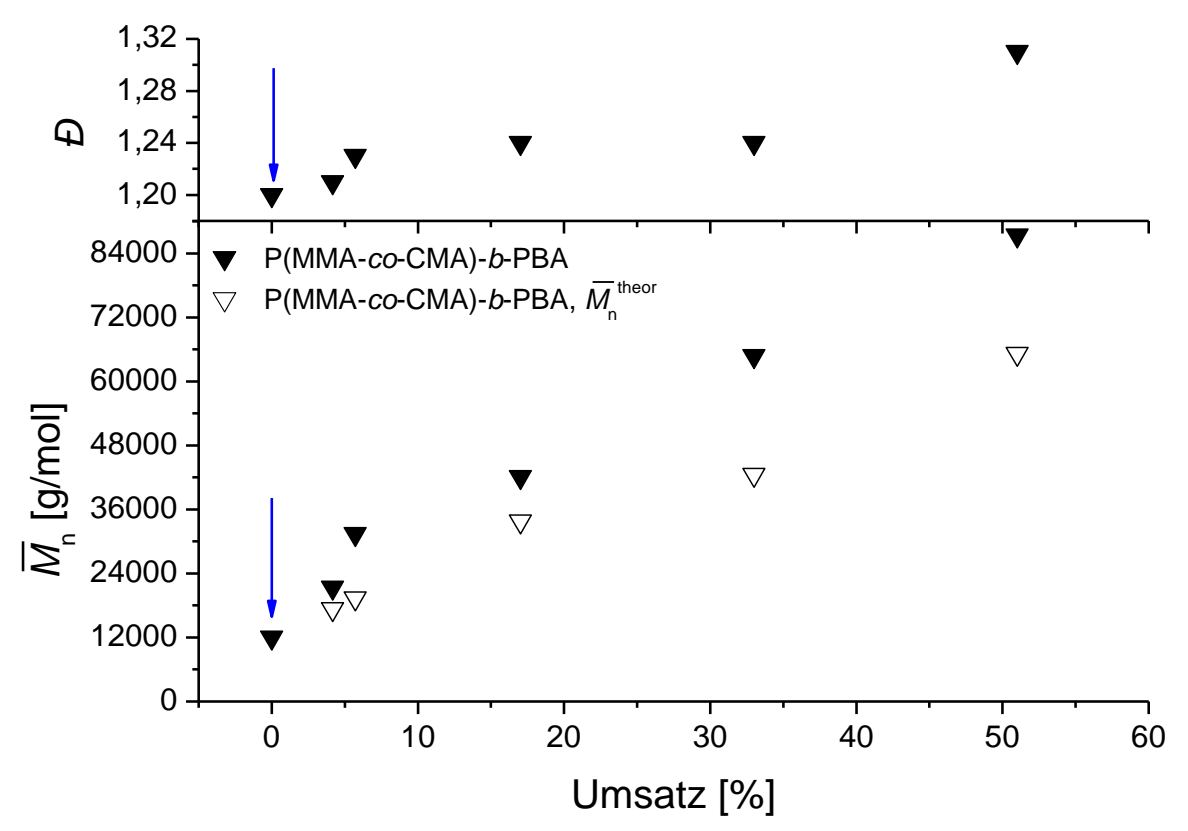

Abbildung 4-14 Entwicklung der Molmasse und der Dispersität in Abhängigkeit vom Monomerumsatz für die Blockcopolymerisation A.

$\mathrm{Zu}$ beachten ist die teilweise starke Abweichung der ermittelten Molmassen (RI-Detektor) der einzelnen Proben von den theoretischen Molmassen. Wie bereits in der Einleitung des Abschnitts 4.2 erwähnt, konnte keine geeignete Kalibrierung für die GPC-Messungen durchgeführt werden, da für das Copolymer-System keine Mark-Houwink-Koeffizienten bekannt sind. Dies stellte auch schon ein Problem für die Molmassenbestimmung des ersten Blocks P(MMA-co-CMA) dar, wobei die Molmassen auf einen PMMA-Standard kalibriert wurden. Um einen geeigneten Vergleich der Molmassen der BCP durchführen zu können, wurde auch für diese Molmassenbestimmung eine PMMA-Kalibrierung verwendet. Dadurch sind die ermittelten Molmassen auch nur als Näherungswerte zu betrachten.

Weiterhin wurden die erhaltenen Blockcopolymere mittels NMR-Spektroskopie untersucht. Abbildung 4-15 zeigt zwei übereinander gelagerte ${ }^{1} \mathrm{H}-\mathrm{NMR}$-Spektren, wobei ein Spektrum zum statistischen Copolymer (schwarz), das den ersten Block bildet, gehört und das zweite Spektrum beispielhaft das Blockcopolymer P(MMA$c o$-CMA)- $b$-PBA zeigt (rot). Durch die zusätzlichen Signale im roten ${ }^{1} \mathrm{H}-\mathrm{NMR}$ Spektrum wird deutlich, dass ein neuer Block (PBA) entstanden ist. Weiterhin sei erwähnt, dass der erste Block das UV-aktive CMA-Monomer enthält, aber in der Strukturformel in Abbildung 4-15 aus übersichtlichen Gründen nicht dargestellt wurde. 


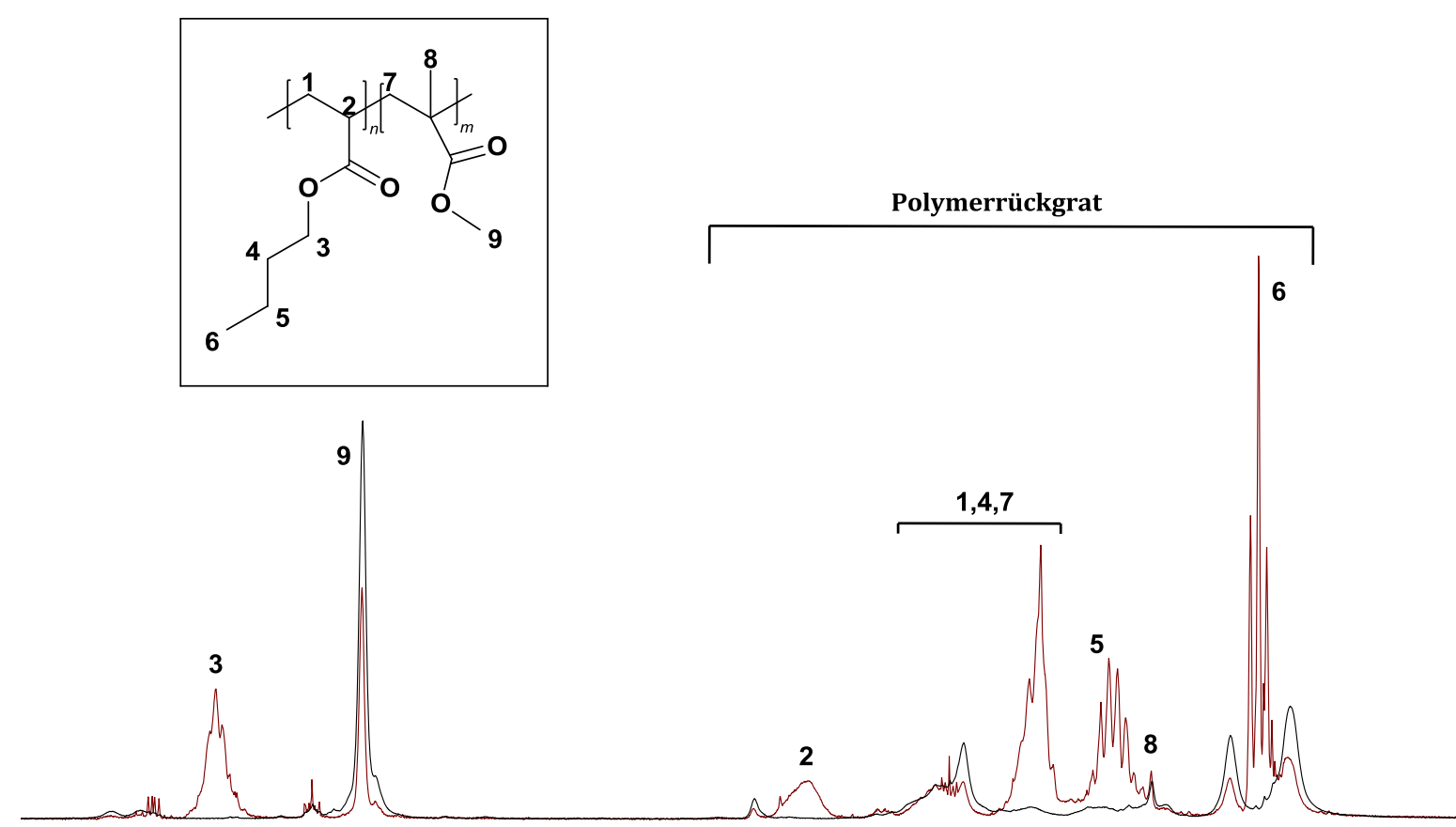

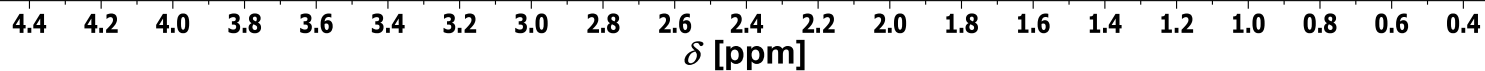

Abbildung 4-15 Übereinandergelegte ${ }^{1} \mathrm{H}-\mathrm{NMR}$-Spektren des makroRAFT-Agens P(MMA-co-CMA) I (schwarz) und des Blockcopolymers P(MMA-co-CMA)- $b$-PBA (rot). Die Spektren wurden in $\mathrm{CDCl}_{3}$ aufgenommen.

Im tiefer verschobenen Bereich des ${ }^{1} \mathrm{H}$-NMR-Spektrums, welcher in Abbildung 4-15 nicht gezeigt wird, sind die aromatischen Signale des CMAs und das Signal für $\mathrm{CDCl}_{3}$ zu sehen, wobei die Signale der UV-aktiven Gruppe nur sehr schwach sind. Bei 4,02 ppm ist das Signal der $\mathrm{CH}_{2}$-Gruppe in Nachbarschaft zur Carbonylgruppe in der BA-Einheit zu sehen. Bei 3,58 ppm ist das Signal der -O- $\mathrm{CH}_{3}-\mathrm{Gruppe}$ der MMA-Einheit zu erkennen. Das Polymerrückgrat erstreckt sich von 0,8 bis 2,3 ppm, wobei in Abbildung 4-15 die einzelnen Signale den jeweiligen Gruppen zugeordnet wurden. Die Signale bei 4,02 und 3,58 ppm wurden integriert, um die Zusammensetzung des BCPs bestimmen zu können. Beide charakteristischen Signale gehören zu den zwei unterschiedlichen Monomeren (MMA, BA), die das Blockcopolymer bilden. Wie in Abschnitt 4.1.1 beschrieben, kann durch die Integration der Signale der molare Anteil der beiden Komponenten im Polymer bestimmt werden $\left(F_{\mathrm{MMA}}\right.$, $\left.F_{\mathrm{BA}}\right)$. Die Integration der Signale für das eingebaute CMA-Monomer konnte aufgrund der schwachen Intensität im ${ }^{1} \mathrm{H}-\mathrm{NMR}$-Spektrum (Abbildung 4-15) nicht durchgeführt werden. Allerdings wurde die Zusammensetzung für das Copolymer P(MMA-co-CMA) zuvor in Abschnitt 4.1.1 bestimmt. Da sich das Verhältnis der 
Copolymer-Einheiten im makroRAFT-Agens während der Polymerisation des BABlocks nicht verändert, kann der molare CMA-Anteil $F_{\mathrm{CMA}}$ im Blockcopolymer durch die Integration der MMA-Signale im ${ }^{1} \mathrm{H}$-NMR-Spektrum (Abbildung 4-15) berechnet werden. Das Verhältnis der molaren Anteile im makroRAFT-Agens I beträgt:

$$
F_{\mathrm{MMA}}: F_{\mathrm{CMA}}=92 \mathrm{~mol} \%: 8 \mathrm{~mol} \%
$$

Daraus folgt:

$$
x_{\mathrm{CMA}}=\frac{8}{92} \cdot \frac{I_{\mathrm{MMA}}}{3}=0,03 .
$$

Die folgenden Gleichungen zeigen beispielhaft die Berechnung der molaren Anteile der Monomere im Polymer nach 20 min.

$$
\begin{gathered}
F_{\mathrm{MMA}}=\frac{\frac{I_{\mathrm{MMA}}}{3}}{\frac{I_{\mathrm{MMA}}}{3}+\frac{I_{\mathrm{BA}}}{2}+x_{\mathrm{CMA}}}=\frac{0,33}{1,13}=0,29=29 \mathrm{~mol} \%, \\
F_{\mathrm{CMA}}=\frac{x_{\mathrm{CMA}}}{\frac{I_{\mathrm{MMA}}}{3}+\frac{I_{\mathrm{BA}}}{2}+x_{\mathrm{CMA}}}=\frac{0,03}{1,13}=0,03=3 \mathrm{~mol} \%, \\
F_{\mathrm{BA}}=\frac{\frac{I_{\mathrm{BA}}}{2}}{\frac{I_{\mathrm{MMA}}}{3}+\frac{I_{\mathrm{BA}}}{2}+x_{\mathrm{CMA}}}=\frac{0,77}{1,13}=0,68=68 \mathrm{~mol} \% .
\end{gathered}
$$

Für die in Abbildung 4-15 dargestellte Blockcopolymerisation A konnte somit die Zusammensetzung der unterschiedlichen Blöcke bestimmt werden. Tabelle 4-10 fasst die ermittelten Ergebnisse zusammen. 
Tabelle 4-10 Übersicht über die Molmassen $\bar{M}_{n}$, Umsatz $X$ und die molaren Anteile $F$ von den Blockcopolymeren der Blockcopolymerisation A.

\begin{tabular}{|l|l|l|l|l|}
\hline Zeit [min] & $\overline{\boldsymbol{M}}_{\mathbf{n}}[\mathbf{g} / \mathbf{m o l}]$ & Umsatz [\%] & $\begin{array}{l}\boldsymbol{F}_{\text {MMA-co-CMA }} \\
{[\mathbf{m o l} \mathbf{0}]}\end{array}$ & $\begin{array}{l}\boldsymbol{F}_{\mathrm{BA}} \\
{[\mathbf{m o l} \%]}\end{array}$ \\
\hline $\mathbf{1 0}$ & 21300 & 4 & 76 & 24 \\
\hline $\mathbf{1 5}$ & 31400 & 6 & 53 & 47 \\
\hline $\mathbf{2 0}$ & 42100 & 17 & 32 & 68 \\
\hline $\mathbf{2 5}$ & 64700 & 33 & 25 & 75 \\
\hline $\mathbf{3 0}$ & 87400 & 51 & 21 & 79 \\
\hline
\end{tabular}

Der molare Anteil von BA im Polymer nimmt mit steigender Reaktionszeit zu. Die Eigenschaften des resultierenden Polymers werden durch beide Polymerblöcke bestimmt, wobei der längere Block den größeren Einfluss hat. Bei der oben beschriebenen Blockcopolymerisation war die Probe mit dem größeren MMAAnteil (10 min) ein gelbes, amorphes Polymer, wobei mit zunehmendem BA-Anteil (nach $20 \mathrm{~min}$ ) ein gelbes, klebriges Polymer entstand.

\section{Variation der makroRAFT-Agenzien für P(MMA-co-CMA)-b-PBA}

In dem folgenden Unterabschnitt werden die Blockcopolymerisationen B und C vorgestellt, wobei verschiedene makroRAFT-Agenzien mit unterschiedlichen Blocklängen (siehe Tabelle 4-9) für die Umsetzung mit dem Monomer BA verwendet wurden. Zuerst wird die Blockcopolymerisation B (Tabelle 4-9) diskutiert, wobei ein im Gegensatz zum makroRAFT-Agens I längerer P(MMA-coCMA)-Block (IV, $\bar{M}_{\mathrm{n}}=47500 \mathrm{~g} / \mathrm{mol}$ ) verwendet wurde. In Abbildung 4-16 sind die Molmassenverteilungen der einzelnen Proben der Blockcopolymerisation B dargestellt. 


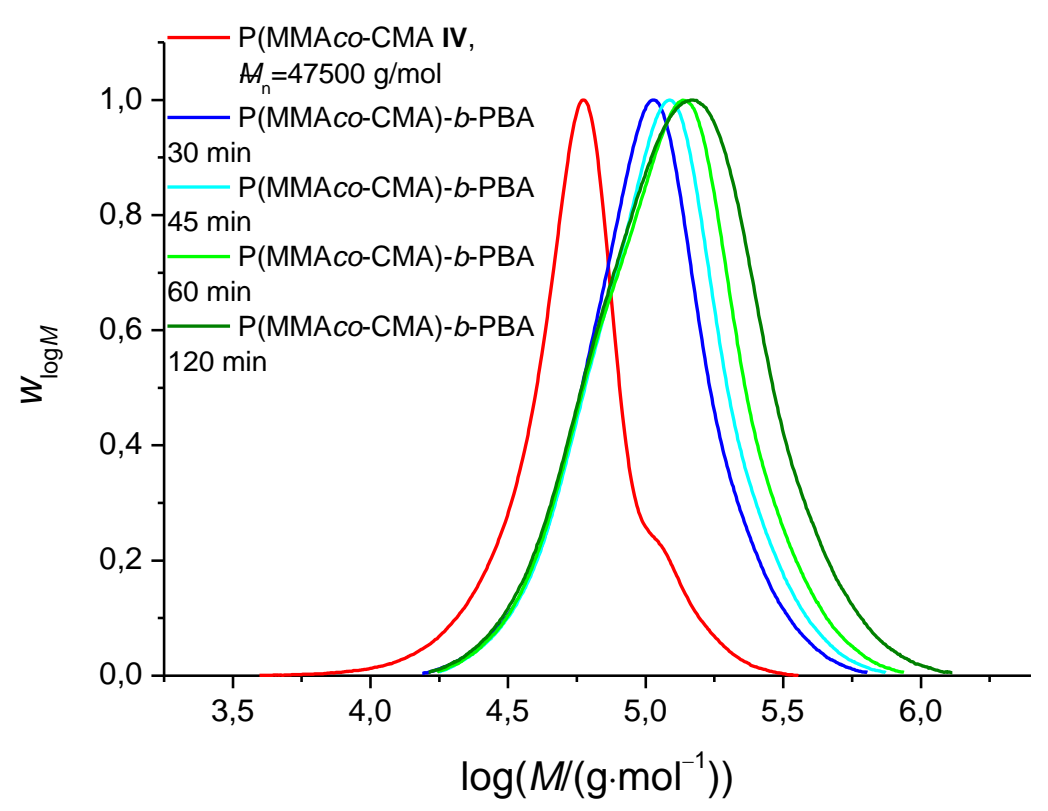

Abbildung 4-16 GP-Chromatogramme (RI-Detektor) von der Blockcopolymersation B, gemessen in THF.

Der in diesem Fall größere Ausgangsblock (IV, $\bar{M}_{\mathrm{n}}=47500 \mathrm{~g} / \mathrm{mol}$ ) zeigt im Gegensatz zu kleineren Blöcken (I, $\bar{M}_{\mathrm{n}}=12000 \mathrm{~g} / \mathrm{mol}$ ) eine niedrigere effektive Konzentration der RAFT-Endgruppen, wobei diese Konzentration entscheidend ist für das weitere Kettenwachstum. Somit werden die MMV der erhaltenen BCP mit zunehmender Reaktionszeit immer breiter. Nebenreaktionen wie z.B. Verzweigung und Terminierung rücken in diesem Fall mehr in den Vordergrund.

Trotz alledem verdeutlichen die Dispersitäten und die Zunahme der Molmassen mit steigendem Monomerumsatz (Abbildung 4-17) eine erfolgreiche Blockcopolymerisation. Die Molmasse und die Dispersität des Ausgangsblocks sind in Abbildung 4-17 mit dem blauen Pfeil bei 0 \% Umsatz gekennzeichnet. 


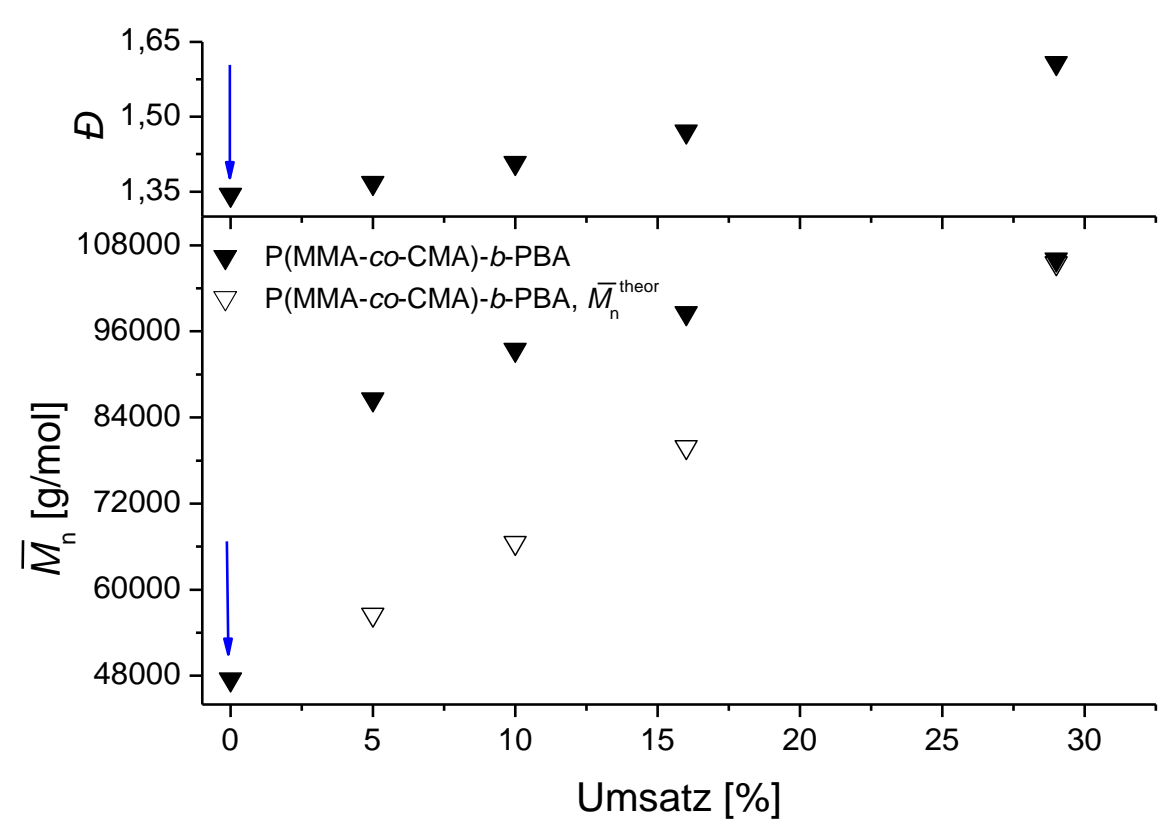

Abbildung 4-17 Entwicklung der Molmasse und der Dispersität in Abhängigkeit vom Monomerumsatz für die Blockcopolymerisation B.

Die ermittelten Molmassen weichen sehr stark von den theoretischen Molmassen ab, wobei die mit der GPC bestimmten Molmassen Näherungswerte darstellen (wie oben beschrieben). Mit zunehmendem Monomerumsatz steigen die Dispersitäten bis auf 1,6. Die höheren Dispersitäten lassen auf ein weniger kontrolliertes Polymerisationsverhalten schließen. Trotzdem verdeutlicht die Zunahme der Molmassen mit steigendem Monomerumsatz eine erfolgreiche Blockcopolymerisation. Die Zusammensetzungen der BCP wurden nach der oben beschriebenen Methode bestimmt und

Tabelle 4-11 fasst die ermittelten Ergebnisse zusammen. Das Verhältnis der molaren Anteile im makroRAFT-Agens IV ist:

$$
F_{\mathrm{MMA}}: F_{\mathrm{CMA}}=93 \mathrm{~mol} \%: 7 \mathrm{~mol} \% \text {. }
$$

Daraus folgt für den CMA-Anteil im Blockcopolymer:

$$
x_{\mathrm{CMA}}=\frac{7}{93} \cdot \frac{I_{\mathrm{MMA}}}{3}=0,03
$$


Tabelle 4-11 Übersicht über die Molmassen $\bar{M}_{n}$, Umsatz $X$ und die molaren Anteile $F$ von den Blockcopolymeren der Blockcopolymerisation B.

\begin{tabular}{|l|l|l|l|l|}
\hline Zeit [min] & $\bar{M}_{\mathbf{n}}[\mathbf{g} / \mathbf{m o l}]$ & Umsatz [\%] & $\begin{array}{l}\boldsymbol{F}_{\text {MMA-co-CMA }} \\
{[\mathbf{m o l} \% \mathbf{c}}\end{array}$ & $\begin{array}{l}\boldsymbol{F}_{\text {BA }} \\
{[\mathbf{m o l} \%]}\end{array}$ \\
\hline $\mathbf{3 0}$ & 86500 & 5 & 61 & 39 \\
\hline $\mathbf{4 5}$ & 93400 & 10 & 54 & 46 \\
\hline $\mathbf{6 0}$ & 98500 & 16 & 48 & 52 \\
\hline $\mathbf{1 2 0}$ & 106000 & 29 & 39 & 61 \\
\hline
\end{tabular}

Die Bestimmung der Polymerzusammensetzung zeigt erneut einen zunehmenden molaren Anteil der BA-Einheiten mit steigender Reaktionszeit. Somit konnten auch BCP mit einem größeren Ausgangsblock erfolgreich synthetisiert werden.

Zusätzlich wurde ein sehr kurzer P(MMA-co-CMA)-Block (III, $\bar{M}_{\mathrm{n}}=5800 \mathrm{~g} / \mathrm{mol}$ ) mit BA umgesetzt. Die Molmassenverteilungen der Blockcopolymerisation C werden in Abbildung 4-18 näher betrachtet. Das rote Chromatogramm stellt das makroRAFT-Agens III mit einer Molmasse von $\bar{M}_{\mathrm{n}}=5800 \mathrm{~g} / \mathrm{mol}$ dar. Die blauen und grünen Chromatogramme zeigen die MMV der einzelnen Proben nach 10, 20 und 60 min. Abbildung 4-19 zeigt den Molmassen- und Dispersitätsverlauf mit dem Monomerumsatz der einzelnen Proben sowie des Ausgangsblocks bei $0 \%$ Umsatz (blauer Pfeil).

Die engen monomodalen Molmassenverteilungen sowie die Zunahme der Molmasse mit steigendem Monomerumsatz verdeutlichen das kontrollierte Verhalten während der Polymerisation. Erst nach $60 \mathrm{~min}$ ist bei der Molmassenverteilung eine kleine Schulter im Bereich zu höheren Molmassen zu sehen, was wie vorher beschrieben auf eine Kettenverzweigung oder Terminierung während der Polymerisation hindeutet. 


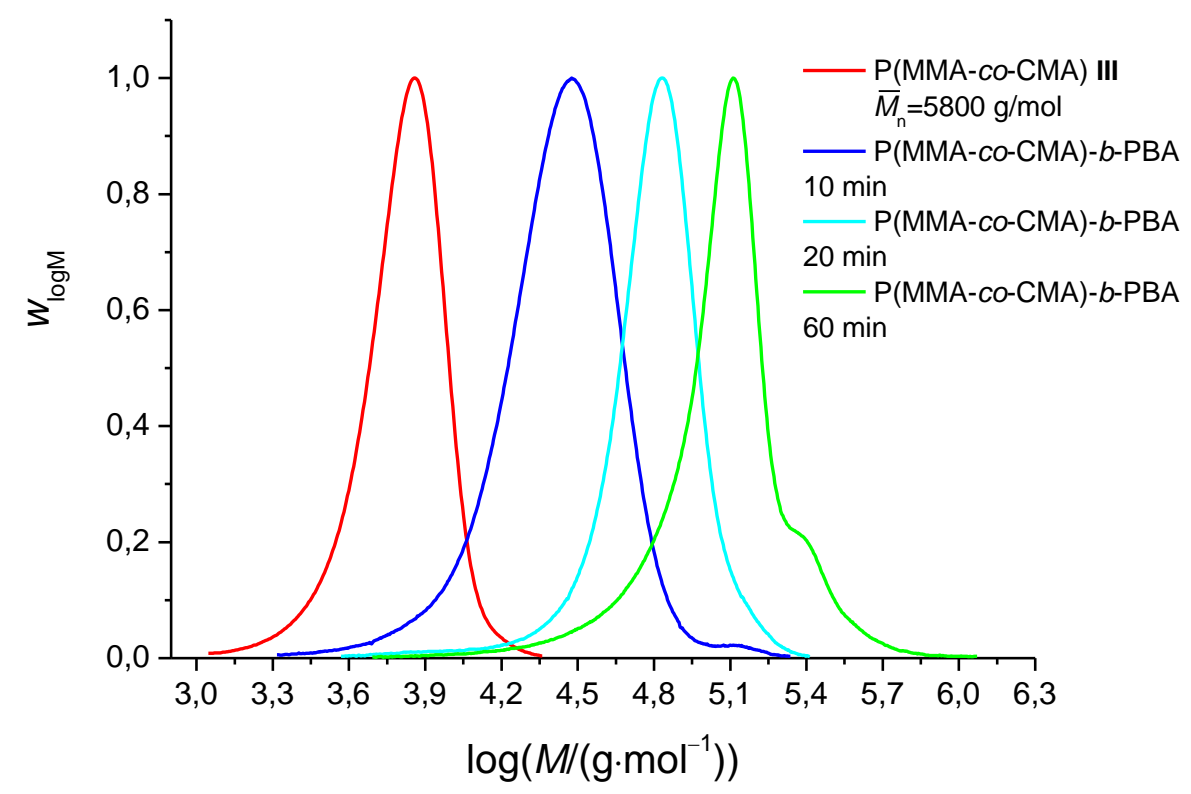

Abbildung 4-18 GP-Chromatogramme (RI-Detektor) von der Blockcopolymersation C, gemessen in THF.

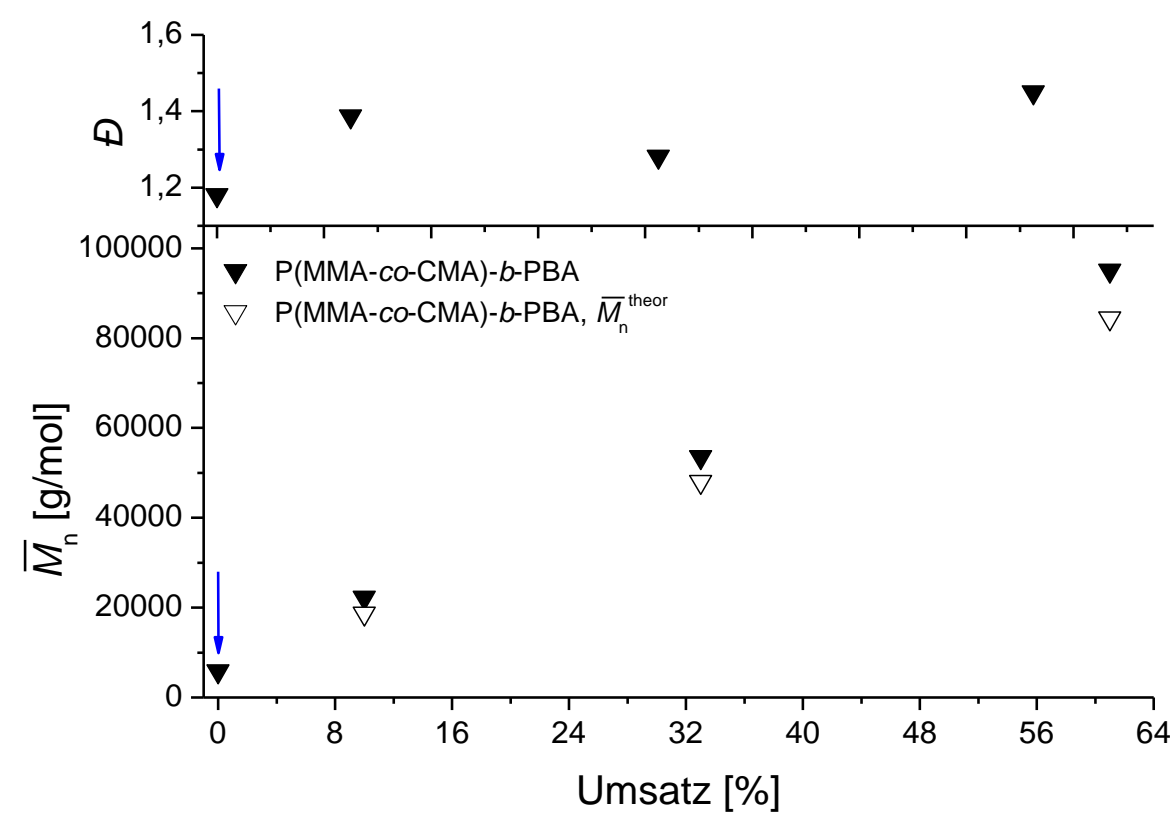

Abbildung 4-19 Entwicklung der Molmasse und der Dispersität in Abhängigkeit vom Monomerumsatz für die Blockcopolymerisation C.

Die Abweichung der ermittelten Molmassen zu den theoretischen Molmassen ist relativ gering im Gegensatz zu den vorangegangen Polymerisationen. Der kurze Ausgangsblock III ( $\left.\bar{M}_{\mathrm{n}}=5800 \mathrm{~g} / \mathrm{mol}\right)$ besitzt eine hohe effektive Konzentration der RAFT-Endgruppen, wodurch ein kontrolliertes Kettenwachstum bis hin zu großen 
Molmassen $\left(\bar{M}_{\mathrm{n}}>53000 \mathrm{~g} / \mathrm{mol}\right)$ gegeben ist. Die Zusammensetzungen der BCP mit kürzerem Ausgangsblock wurden mittels NMR-Spektroskopie analog zu der oben beschriebenen Methode bestimmt und die Ergebnisse sind in Tabelle 4-12 zusammengefasst. Das Verhältnis der molaren Anteile $F_{\mathrm{MMA}}$ und $F_{\mathrm{CMA}}$ im makroRAFT-Agens III und IV sind identisch (siehe Tabelle 4-8).

Tabelle 4-12 Übersicht über die Molmassen $\bar{M}_{\mathrm{n}}$, Umsatz $X$ und die molaren Anteile $F$ von den Blockcopolymeren der Blockcopolymerisation C.

\begin{tabular}{|l|l|l|l|l|}
\hline Zeit [min] & $\bar{M}_{\mathbf{n}}[\mathbf{g} / \mathbf{m o l}]$ & Umsatz [\%] & $\begin{array}{l}\boldsymbol{F}_{\text {MMA-co-CMA }} \\
{[\mathbf{m o l} \%]}\end{array}$ & $\begin{array}{l}\boldsymbol{F}_{\mathrm{BA}} \\
{[\mathbf{m o l} \%]}\end{array}$ \\
\hline $\mathbf{1 0}$ & 22200 & 10 & 31 & 69 \\
\hline $\mathbf{2 0}$ & 53500 & 33 & 14 & 86 \\
\hline $\mathbf{6 0}$ & 95100 & 61 & 7 & 93 \\
\hline
\end{tabular}

Fazit

Insgesamt konnte in diesem Abschnitt die erfolgreiche Blockcopolymerisation von MMA und BA mittels RAFT-Polymerisation dargestellt werden. Die synthetisierten Blockcopolymersysteme variieren in ihren einzelnen Blocklängen und somit in der Zusammensetzung der Blöcke im Polymer sowie in der Molmasse der Blockcopolymere. Weiterhin konnte in allen Blockcopolymeren ein erfolgreicher Einbau von UV-aktiven Einheiten gezeigt werden.

\subsubsection{Herstellung von P(MMA-co-CMA)-b-PBMA}

Die beiden Monomere des vorangegangenen Polymer-Systems sind in ihrer Art recht unterschiedlich. Das Monomer im ersten Block gehört zu der Gruppe der Methacrylate und das Monomer im zweiten Block gehört zu der Acrylat-Gruppe. In diesem Abschnitt soll ein weiteres Blockcopolymersystem synthetisiert werden, bei dem die Monomere in beiden Blöcken zur Methacrylat-Gruppe gehören. Aufgrund der Ähnlichkeit der Monomereinheiten wird eine geringere Entmischungstendenz der hergestellten BCP angenommen und es besteht die Möglichkeit der Bestimmung des Ordnungs-Unordnungs-Übergangs für das Blockcopolymersystem z. B. durch die Variation der Temperatur.

Für die folgende Polymerisation wurde das makroRAFT-Agens II mit einer Molmasse von $\bar{M}_{\mathrm{n}}=14300 \mathrm{~g} / \mathrm{mol}$ verwendet. Die Zusammensetzung der 
Polymerisationsmischung ist in Tabelle 4-13 aufgeführt (siehe auch Anhang A, Tabelle A-12).

Tabelle 4-13 Übersicht über die Zusammensetzung der Polymerisationsmischung für die Blockcopolymerisationen von BMA mit dem makroRAFT-Agens II (siehe Tabelle 4-8). Die Polymerisation wurde in Toluol bei $70^{\circ} \mathrm{C}$ durchgeführt.

\begin{tabular}{|l|c|l|l|l|}
\hline Polymerisation & Molverhältnis & $\begin{array}{l}\text { makroRAFT- } \\
\text { Agens }\end{array}$ & $\begin{array}{l}\boldsymbol{c}_{\mathrm{BA}} \\
\text { [mol/L] }\end{array}$ & $\begin{array}{l}\boldsymbol{c}_{\mathrm{AIBN}} \\
{[\mathbf{m m o l} / \mathbf{L}]}\end{array}$ \\
\hline $\begin{array}{l}\text { Blockcopolymerisation } \\
\text { D }\end{array}$ & $\begin{array}{c}\text { BA:makroRAFT:AIBN } \\
=\end{array}$ & II & 3,5 & 1 \\
& $1000: 1: 0,2$ & & & \\
\hline
\end{tabular}

Wie bei den Blockcopolymerisationen aus dem vorherigen Abschnitt 4.2.1 sind für dieses Copolymer-System keine Standard-Parameter für die Kalibrierung der GPC verfügbar. Deshalb wurde die GPC für die Bestimmung der Molmassen mit einem PMMA-Standard kalibriert, der auch schon für die Molmassenbestimmung des Ausgangsblocks P(MMA-co-CMA) verwendet wurde. Die Molmassenverteilungen der einzelnen Proben der Blockcopolymerisation D sind in Abbildung 4-20 dargestellt, wobei das rote Chromatogramm den Ausgangsblock II $\left(\bar{M}_{\mathrm{n}}=14300 \mathrm{~g} / \mathrm{mol}\right)$ zeigt. Abbildung 4-20 stellt eine Zunahme der Molmassen mit dem Monomerumsatz dar.

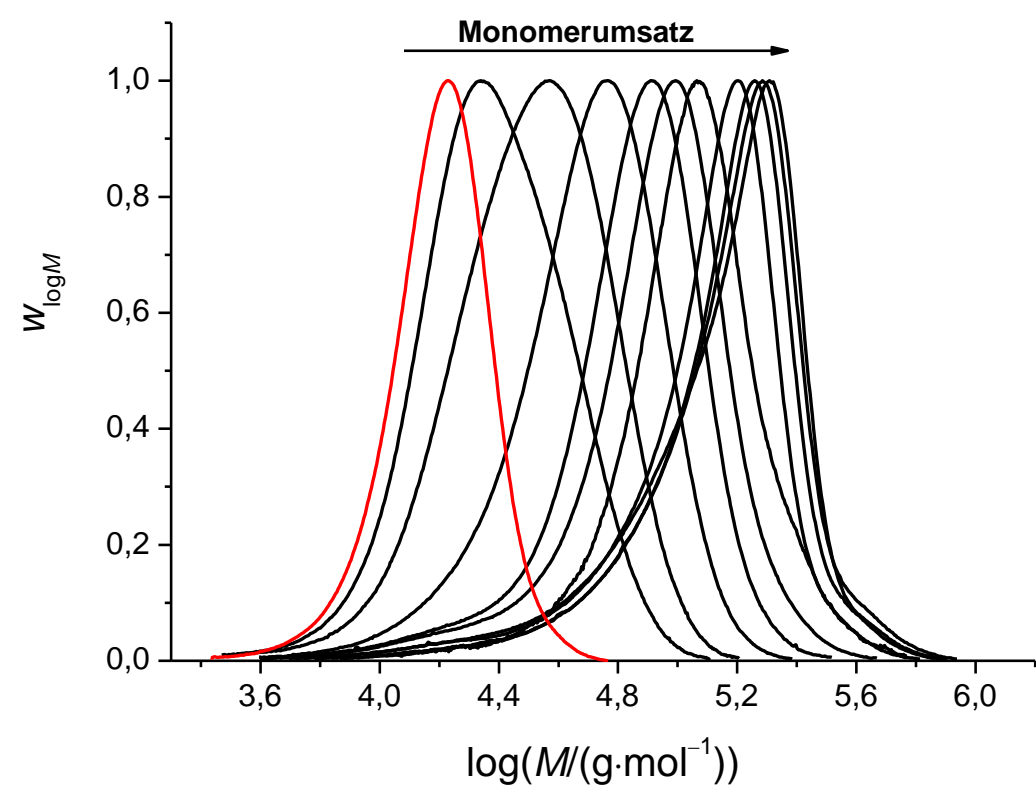

Abbildung 4-20 GP-Chromatogramme (RI-Detektor) von der Blockcopolymersation D, gemessen in THF. 
Abbildung 4-21 zeigt die Entwicklung der Molmasse und Dispersität mit zunehmenden Monomerumsatz für die Blockcopolymerisation D. Der blaue Pfeil veranschaulicht den Ausgangsblock II bei 0 \% Umsatz.

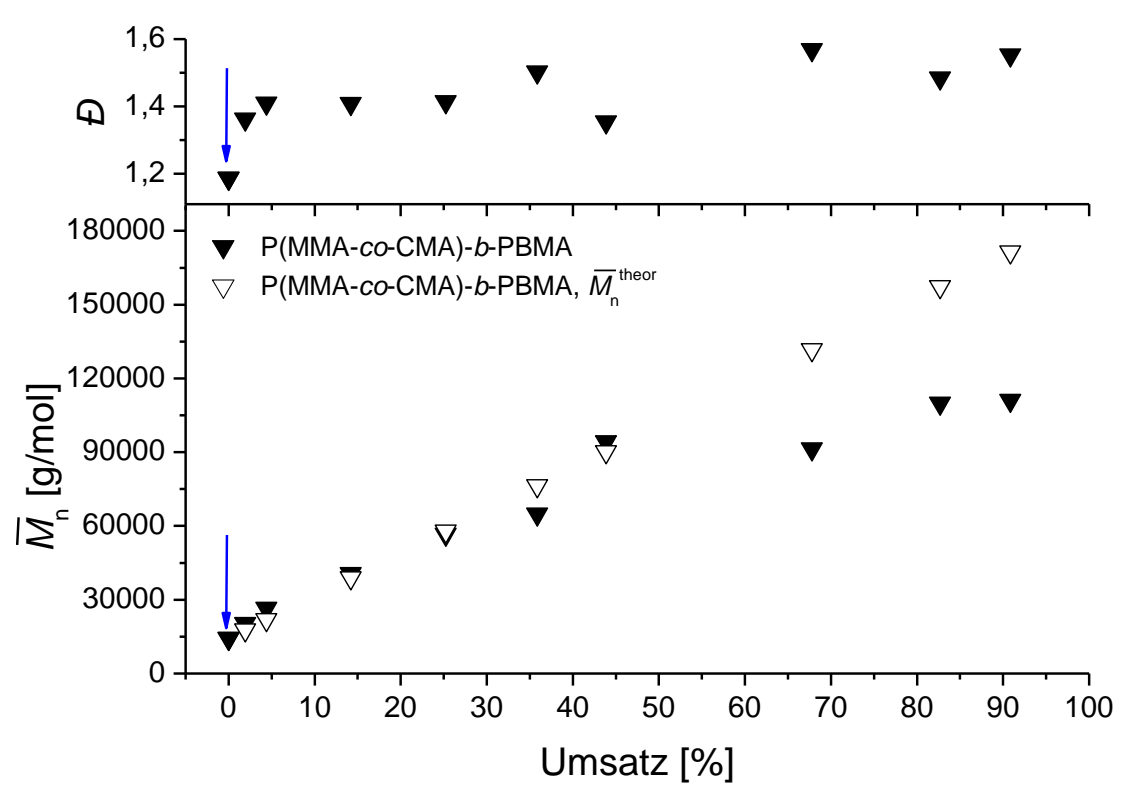

Abbildung 4-21 Entwicklung der Molmasse und der Dispersität in Abhängigkeit vom Monomerumsatz für die Blockcopolymerisation D.

Abgesehen von den etwas erhöhten Dispersitäten (oberer Teil in Abbildung 4-21), kann durch den linearen Verlauf der Molmassen mit zunehmendem Monomerumsatz ein kontrolliertes Polymerisationsverhalten festgestellt werden. Die ermittelten Molmassen stehen in guter Übereinstimmung mit den theoretischen Molmassen, wobei mit zunehmenden Monomerumsatz $(X>70 \%)$ die Abweichung zwischen theoretischer und experimentell bestimmter Molmasse stark zunimmt. Diese Abweichung sowie die erhöhten Dispersitäten deuten auf eine Abnahme der Polymerisationskontrolle ab einem Monomerumsatz von ca. $70 \%$, was auf Zunahme der Nebenreaktionen wie z.B. Terminierung zurückzuführen ist.

Weiterhin wurden die erhaltenen Blockcopolymere mittels NMR-Spektroskopie untersucht. Abbildung 4-22 zeigt zwei übereinander gelegte ${ }^{1} \mathrm{H}-\mathrm{NMR}$-Spektren, wobei das schwarze Spektrum das Ausgangsmaterial und das rote Spektrum beispielhaft ein BCP mit BMA widerspiegelt. Durch die zusätzlichen Signale im roten ${ }^{1} \mathrm{H}$-NMR-Spektrum wird deutlich, dass ein neuer Block (PBMA) entstanden ist. Weiterhin sei erwähnt, dass der erste Block das UV-aktive CMA-Monomer 
enthält, aber in der Strukturformel in Abbildung 4-22 aus übersichtlichen Gründen nicht dargestellt wurde.

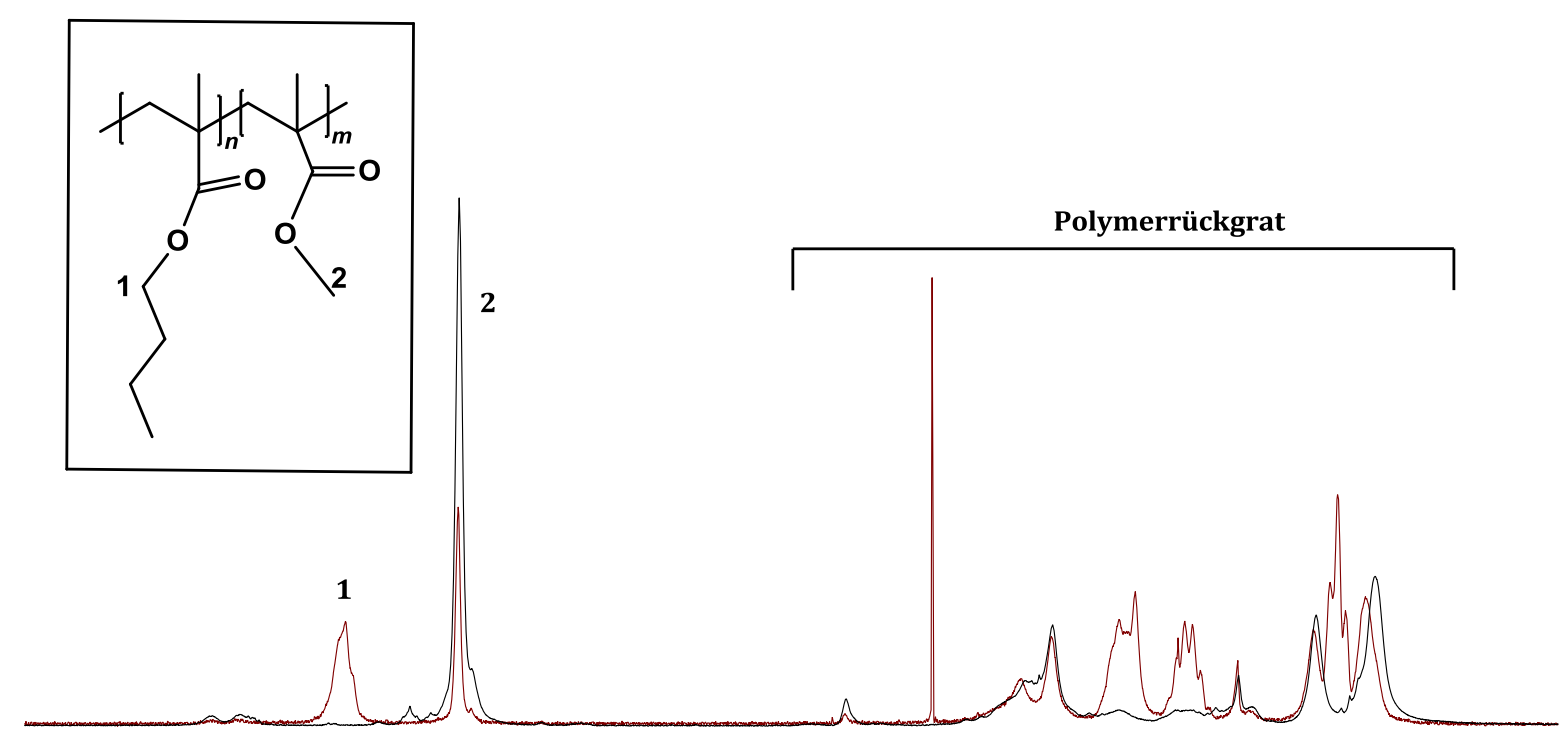

\begin{tabular}{llllllllllllllllllllllllll}
\hline 4.8 & 4.6 & 4.4 & 4.2 & 4.0 & 3.8 & 3.6 & 3.4 & 3.2 & 3.0 & 2.8 & 2.6 & 2.4 & 2.2 & 2.0 & 1.8 & 1.6 & 1.4 & 1.2 & 1.0 & 0.8 & 0.6 & 0.4
\end{tabular}

Abbildung 4-22 Übereinandergelegte ${ }^{1} \mathrm{H}$-NMR-Spektren von dem makroRAFT-Agens P(MMA-coCMA) II (schwarz) und dem BCP P(MMA-co-CMA)- $b$-PBMA (rot). Die Spektren wurden in $\mathrm{CDCl}_{3}$ aufgenommen.

Im tiefer verschobenen Bereich des ${ }^{1} \mathrm{H}$-NMR-Spektrums, welcher in Abbildung 4-22 nicht gezeigt wird, sind die aromatischen Signale des CMAs und das Signal für $\mathrm{CDCl}_{3}$ zu sehen, wobei die Signale der UV-aktiven Gruppe nur sehr schwach sind. Bei 3,92 ppm ist das Signal der -O- $\mathrm{CH}_{2}$-Gruppe der BMA-Einheit zu sehen. Bei 3,58 ppm ist das Signal der -O- $\mathrm{CH}_{3}$-Gruppe der MMA-Einheit zu erkennen. Das Polymerrückgrat erstreckt sich von 0,85 bis 2,1 ppm. Die Signale bei 3,92 und 3,58 ppm wurden integriert, um die Zusammensetzung des BCPs bestimmen zu können. Beide charakteristischen Signale gehören zu den zwei unterschiedlichen Monomeren, die das BCP bilden. Wie in Abschnitt 4.1.1 beschrieben, kann durch die Integration der Signale der molare Anteil der beiden Komponenten im Polymer bestimmt werden $\left(F_{\mathrm{MMA}}, F_{\mathrm{BMA}}\right)$. Die berechneten Ergebnisse für drei ausgewählte Proben der Blockcopolymerisation D sind in Tabelle 4-14 zusammengefasst. Das Verhältnis der molaren Anteile im makroRAFT-Agens II ist: 
$F_{\mathrm{MMA}}: F_{\mathrm{CMA}}=93 \mathrm{~mol} \%: 7 \mathrm{~mol} \%$.

Daraus folgt für den CMA-Anteil im Blockcopolymer:

$$
x_{\mathrm{CMA}}=\frac{7}{93} \cdot \frac{I_{\mathrm{MMA}}}{3}=0,03
$$

Tabelle 4-14 Übersicht über die Molmassen $\bar{M}_{\mathrm{n}}$, Umsatz $X$ und die molaren Anteile $F$ von drei Proben der Blockcopolymerisation D.

\begin{tabular}{|l|l|l|l|l|}
\hline Zeit [h] & $\bar{M}_{\mathbf{n}}$ [g/mol] & Umsatz [\%] & $\begin{array}{l}\boldsymbol{F}_{\text {MMA-co-CMA }} \\
{[\mathbf{m o l} \% \mathbf{l}}\end{array}$ & $\begin{array}{l}\boldsymbol{F}_{\text {BMA }} \\
{[\mathbf{m o l} \%]}\end{array}$ \\
\hline $\mathbf{1}$ & 40600 & 14 & 36 & 64 \\
\hline $\mathbf{4}$ & 94200 & 44 & 17 & 83 \\
\hline $\mathbf{2 9}$ & 100200 & 90 & 9 & 91 \\
\hline
\end{tabular}

Der molare Anteil von BMA im Polymer nimmt mit steigender Reaktionszeit zu. Die Eigenschaften des resultierenden Polymers werden durch beide Polymerblöcke bestimmt. Das entstandene Blockcopolymer ist ein weißes, amorphes Material, das mit zunehmendem BMA-Anteil härter wird.

Zusammenfassend konnte festgestellt werden, dass die Blockcopolymerisation von MMA und BMA mittels RAFT-Polymerisation erfolgreich durchgeführt werden kann. In dem entstandenen Blockcopolymersystem werden beide Blöcke aus Monomeren der Methacrylat-Gruppe gebildet. Die Reaktion findet in einem angemessenen Zeitintervall statt und kann somit als geeigneter Weg für den Einbau UV-aktiver Gruppen in das Polymer genutzt werden. 


\subsection{3 Übersicht verwendeter Blockcopolymere}

In den folgenden Abschnitten wird die Analyse der unterschiedlichen Blockcopolymersysteme erläutert. Für eine bessere Übersicht werden die verschiedenen Polymersysteme mit römischen Ziffern abgekürzt. Tabelle 4-15 stellt die unterschiedlichen BCP mit ihren dazugehörigen makroRAFT-Agenzien P(MMA-co-CMA) dar. Die makroRAFT-Agenzien wurden in Abschnitt 4.1.4 in Tabelle 4-8 zusammengefasst.

Tabelle 4-15 Übersicht über die Molmassen $\bar{M}_{\mathrm{n}}$, Dispersitäten $\oslash$, molaren Anteile $F$ von den synthetisierten BCP P(MMA-co-CMA)- $b$-PBA und P(MMA-co-CMA)- $b$-PBMA sowie die Anzahl der BA- und BMA-Einheiten im jeweiligen BCP.

\begin{tabular}{|c|c|c|c|c|c|c|}
\hline Probe & $\begin{array}{l}\text { makro- } \\
\text { RAFT- } \\
\text { Agens }\end{array}$ & $\begin{array}{l}\bar{M}_{\mathrm{n}} \\
{[\mathrm{g} / \mathrm{mol}]}\end{array}$ & $\boldsymbol{\oplus}$ & $\begin{array}{l}F_{\text {MMA-co- }} \\
\text { CMA } \\
{[\mathrm{mol} \%]}\end{array}$ & $\begin{array}{l}F_{\mathrm{BA}} \\
{[\mathrm{mol} \%]}\end{array}$ & $\begin{array}{l}\text { Anzahl } \\
\text { der BA- } \\
\text { Einheiten }\end{array}$ \\
\hline $\begin{array}{l}\text { V }\left[\mathrm{P}\left(\mathrm{MMA}_{390}-\mathrm{CO}-\right.\right. \\
\left.\left.\mathrm{CMA}_{29}\right)-b-\mathrm{PBA}\right]_{426}\end{array}$ & IV & 98500 & 1,47 & 48 & 52 & 426 \\
\hline $\begin{array}{l}\text { VI }\left[\mathrm{P}\left(\mathrm{MMA}_{390}-c o-\right.\right. \\
\left.\left.\mathrm{CMA}_{29}\right)-b-\mathrm{PBA}\right]_{526}\end{array}$ & IV & 106000 & 1,61 & 39 & 61 & 526 \\
\hline $\begin{array}{l}\text { VII }\left[\mathrm{P}\left(\mathrm{MMA}_{95}-\mathrm{co}-\right.\right. \\
\left.\left.\mathrm{CMA}_{9}\right)-b-\mathrm{PBA}\right]_{229}\end{array}$ & I & 42100 & 1,24 & 32 & 68 & 229 \\
\hline $\begin{array}{l}\text { VIII }\left[\mathrm{P}\left(\mathrm{MMA}_{95}-\mathrm{co}-\right.\right. \\
\left.\left.\mathrm{CMA}_{9}\right)-b-\mathrm{PBA}\right]_{388}\end{array}$ & I & 64700 & 1,24 & 25 & 75 & 388 \\
\hline $\begin{array}{l}\text { IX }\left[\mathrm{P}\left(\mathrm{MMA}_{95}-\mathrm{co}-\right.\right. \\
\left.\left.\mathrm{CMA}_{9}\right)-b-\mathrm{PBA}\right]_{552}\end{array}$ & I & 87400 & 1,31 & 21 & 79 & 552 \\
\hline $\begin{array}{l}\mathbf{X}\left[\mathrm{P}\left(\mathrm{MMA}_{48}-\mathrm{CO}-\mathrm{CMA}_{4}\right)-\right. \\
b-\mathrm{PBA}]_{124}\end{array}$ & III & 22200 & 1,39 & 31 & 69 & 124 \\
\hline Probe & $\begin{array}{l}\text { makro- } \\
\text { RAFT- } \\
\text { Agens }\end{array}$ & $\begin{array}{l}\bar{M}_{\mathrm{n}} \\
{[\mathrm{g} / \mathrm{mol}]}\end{array}$ & $\boldsymbol{D}$ & $\begin{array}{l}F_{\text {MMA-co- }} \\
\text { CMA } \\
{[\mathrm{mol} \%]}\end{array}$ & $\begin{array}{l}F_{\text {BMA }} \\
{[\mathrm{mol} \%]}\end{array}$ & $\begin{array}{l}\text { Anzahl } \\
\text { der BMA- } \\
\text { Einheiten }\end{array}$ \\
\hline $\begin{array}{l}\text { XI }\left[\mathrm{P}\left(\mathrm{MMA}_{117}-\mathrm{Co}-\right.\right. \\
\left.\left.\mathrm{CMA}_{9}\right)-b-\mathrm{PBMA}\right]_{177}\end{array}$ & II & 40600 & 1,41 & 36 & 64 & 177 \\
\hline $\begin{array}{l}\text { XII [P(MMA }{ }_{117}-c o- \\
\left.\left.\text { CMA }_{9}\right)-b-P B M A\right]_{635}\end{array}$ & II & 100200 & 1,67 & 9 & 91 & 635 \\
\hline
\end{tabular}




\subsection{Thermisches Verhalten}

Das thermische Verhalten der BCP wurde mit dynamisch-mechanischer Analyse (DMA, siehe Abschnitt 2.6) bestimmt, um Aussagen zum Phasenverhalten treffen zu können. Zusätzlich wurde die thermische Stabilität der BCP mittels thermogravimetrischer Analyse (TGA) untersucht. Die Ergebnisse der Messungen ergaben, dass die BCP sich ab einer Temperatur von $T>350^{\circ} \mathrm{C}$ zersetzen. Abbildung 4-23 zeigt beispielhaft ein mit TGA aufgenommenes Thermogramm von der Probe VII.

Somit konnten die in dieser Arbeit durchgeführten Temper- und Heizprozesse bei Temperaturen bis zu $250{ }^{\circ} \mathrm{C}$ durchgeführt werden, ohne ein Risiko der Zersetzung einzugehen. Es ist zu erwähnen, dass die DMA-Messungen im Gegensatz zu den TGA-Untersuchungen unter Umgebungsbedingungen (Luft) durchgeführt wurden, wodurch sich die Zersetzungstemperatur leicht verringern kann. Allerdings wurde die Temperaturspanne für die gesamten Messungen ausreichend groß gewählt.

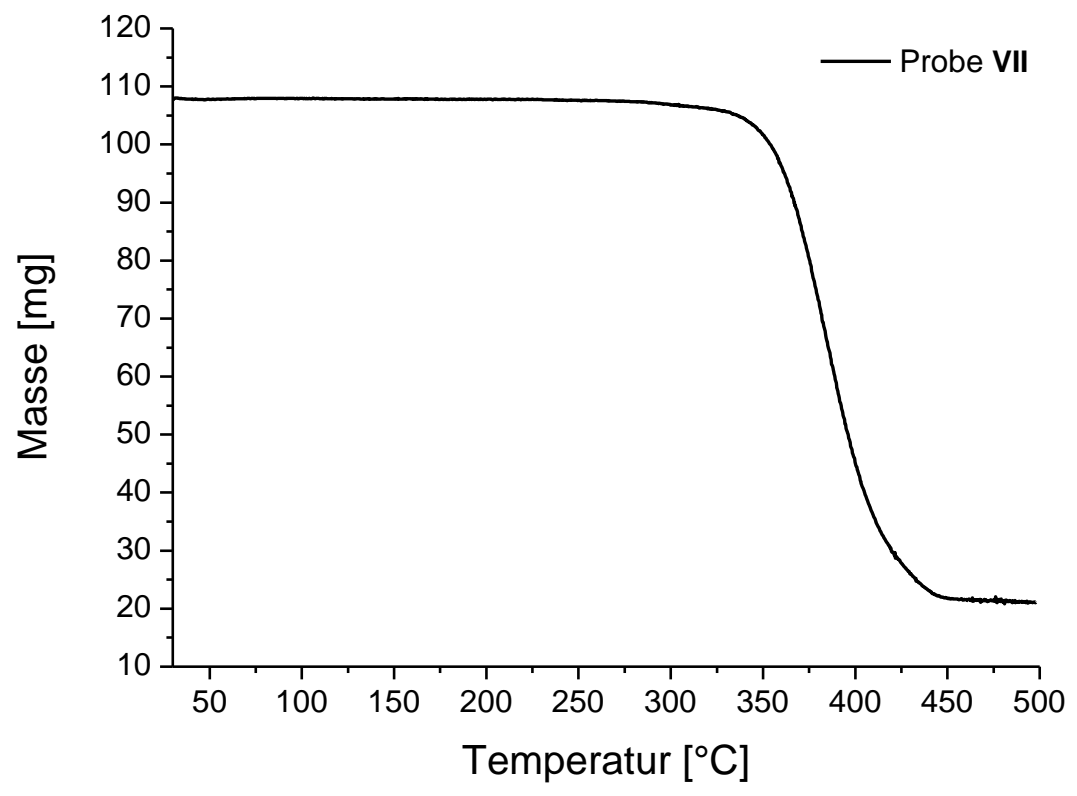

Abbildung 4-23 Thermogramm von Probe VII.

Vor der Bestimmung der Glasübergangstemperaturen der BCP erfolgte die Untersuchung der beiden Polymere P(PMMA-co-CMA) und PBA. Die bestimmte Glasübergangstemperatur des Homopolymers PBA liegt bei $T_{\mathrm{g}}=-40{ }^{\circ} \mathrm{C}$, wobei dieser Wert gut mit der Literatur übereinstimmt. ${ }^{164}$ Der Literaturwert für PMMA 
liegt bei $T_{\mathrm{g}} \approx 105^{\circ} \mathrm{C} .164$ Da für die Synthese der BCP unterschiedliche makroRAFTAgenzien verwendet wurden, sind die ermittelten Glasüberganstemperaturen $T_{\mathrm{g}, 1}$ für die P(MMA-co-CMA)-Blöcke mit den dazugehörigen Molmassen und Dispersitäten in der Tabelle 4-16 dargestellt. Probe II wurde aufgrund der Ähnlichkeit zu Probe I nicht separat untersucht, da davon ausgegangen werden kann, dass beide Proben eine ähnliche $T_{\mathrm{g}}$ aufweisen. Insgesamt konnte für alle makroRAFT-Agenzien eine Glasübergangstemperatur ermittelt werden, was vermuten lässt, dass das CMA statistisch eingebaut wurde. Der höhere $T_{\mathrm{g}}$-Wert für die höhere Molmasse bei Probe IV, sowie die niedrigere $T_{\mathrm{g}}$ bei niedrigerer Molmasse bei Probe III waren für lineare Polymere durch die Fox-Flory-Theorie zu erwarten. Dabei erhöhen z. B. niedrigere Molmassen die Kettenbeweglichkeit und verringern somit die $T_{\mathrm{g}}$.

Tabelle 4-16 Übersicht über Glasübergangstemperaturen $T_{\mathrm{g}, 1}$ der synthetisierten makroRAFTAgenzien P(MMA-co-CMA) mit den dazugehörigen Molmassen $\bar{M}_{\mathrm{n}}$ und den Dispersitäten $\oslash$.

\begin{tabular}{|l|l|l|l|}
\hline Probe & $\left.\overline{\boldsymbol{M}}_{\mathbf{n}} \mathbf{[ g} / \mathbf{m o l}\right]$ & $\boldsymbol{D}$ & $\left.\boldsymbol{T}_{\mathbf{g}, \mathbf{1}}{ }^{\circ} \mathbf{C}\right]$ \\
\hline I & 12000 & 1,20 & 109 \\
\hline III & 5800 & 1,18 & 88 \\
\hline IV & 47500 & 1,30 & 120 \\
\hline
\end{tabular}

Zur Bestimmung der Glasübergangstemperaturen $T_{\mathrm{g}}$ der einzelnen Blöcke wurden die BCP mit dem Gerät DMA 8000 untersucht, wobei Informationen zur Durchführung dem Abschnitt $6.5 \mathrm{zu}$ entnehmen sind. Die ermittelten Werte sind in Tabelle 4-17 zusammengefasst und zeigen für alle Proben zwei deutliche Glasübergangstemperaturen, wobei $T_{\mathrm{g}, 1}$ den P(MMA-co-CMA)-Block repräsentiert und $T_{\mathrm{g}, 2}$ den PBA-Block. Zusätzlich wird in Abbildung 4-24 eine beispielhafte Messkurve gezeigt.

Tabelle 4-17 Übersicht über Glasübergangstemperaturen der synthetisierten BCP P(MMA-coCMA)- $b$-PBA mit den dazugehörigen Molmassen $\bar{M}_{\mathrm{n}}$, Dispersitäten $Ð$ und molaren Anteil $F_{\mathrm{BA}}$.

\begin{tabular}{|l|l|l|l|l|l|}
\hline Probe & $\overline{\boldsymbol{M}}_{\mathbf{n}}[\mathbf{g} / \mathbf{m o l}]$ & $\boldsymbol{D}$ & $\boldsymbol{F}_{\mathrm{BA}}[\mathbf{m o l} \mathbf{0}]$ & $\boldsymbol{T}_{\mathbf{g}, \mathbf{1}}\left[{ }^{\circ} \mathbf{C}\right]$ & $\boldsymbol{T}_{\mathbf{g}, 2}\left[{ }^{\circ} \mathbf{C}\right]$ \\
\hline $\mathbf{V}$ & 98500 & 1,47 & 52 & 80 & -36 \\
\hline VI & 106000 & 1,61 & 61 & 73 & -37 \\
\hline VII & 42100 & 1,24 & 68 & 62 & -36 \\
\hline VIII & 64700 & 1,24 & 75 & 69 & -38 \\
\hline IX & 87400 & 1,31 & 79 & 54 & -39 \\
\hline $\mathbf{X}$ & 22200 & 1,39 & 69 & 140 & -36 \\
\hline
\end{tabular}


Mit zunehmendem PBA-Anteil nehmen die $T_{\mathrm{g}}$-Werte der Proben $\mathbf{V}$ bis IX sowohl für den PBA-Block als auch für den P(MMA-co-CMA)-Block ab. Im Vergleich zu den Homopolymeren sind die Glasübergangstemperaturen der Blöcke leicht aufeinander zu verschoben, wobei dies ein literaturbekannter Effekt ist. ${ }^{165}$ Es kann von beiden Komponenten ein Teil der Ketten in die jeweils andere Phase übergehen, was im Falle des $T_{\mathrm{g}}$-Werts des PBAs zu einer Erhöhung (in Richtung PMMA) und im Falle des P(MMA-co-CMA)-Blocks zu einer Verringerung der $T_{\mathrm{g}}$ (in Richtung PBA) führt. Die Bestimmung von zwei Glasübergangstemperaturen bei den synthetisierten BCP ist ein eindeutiges Indiz für MPS, sodass davon ausgegangen werden kann, dass die Proben phasensepariert vorliegen.

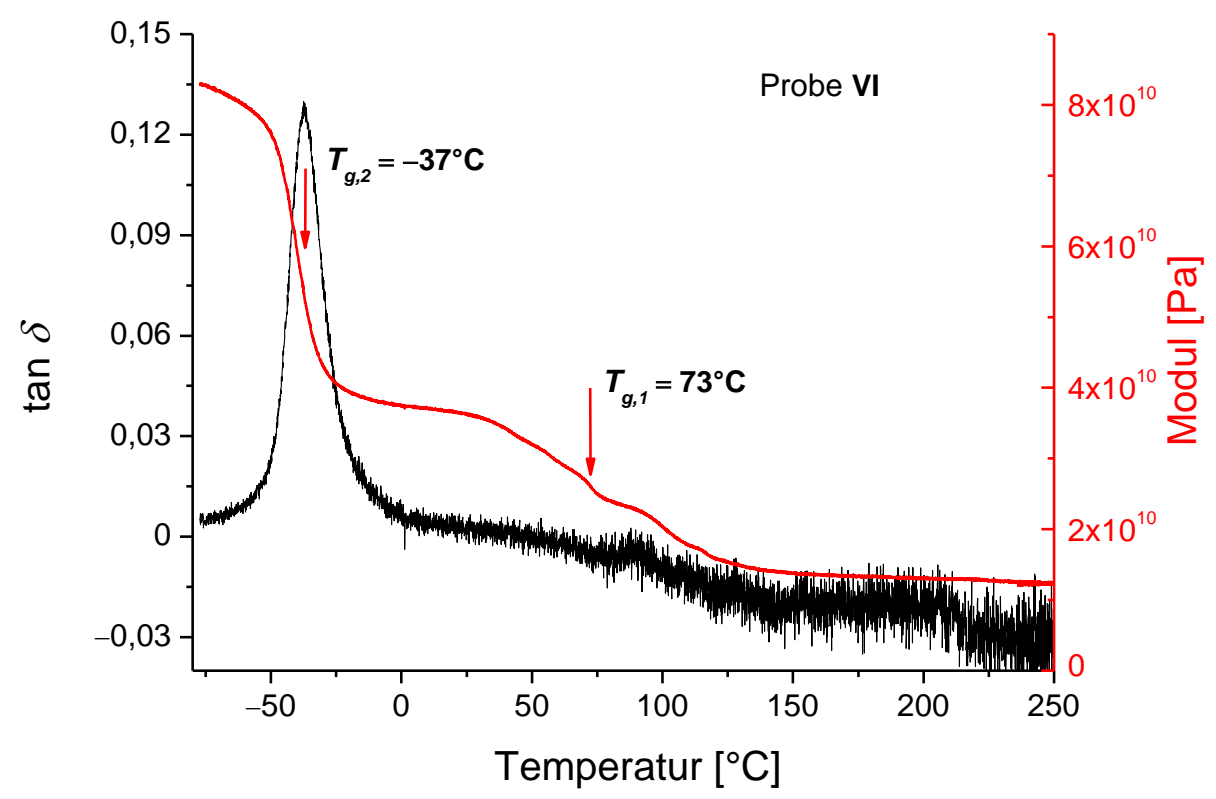

Abbildung 4-24 Darstellung der Glasübergangstemperaturen von Probe VI in Abhängigkeit von dem temperaturabhängigen $\tan \delta$ und der Modulbestimmung. $T_{\mathrm{g}, 1}$ repräsentiert den P(MMA-coCMA)-Block und $T_{\mathrm{g}, 2}$ den PBA-Block.

Im Verlauf der vorliegenden Arbeit wurde bereits darauf hingewiesen, dass eine Mischung der einzelnen Komponenten des BCP-Systems bei höheren Temperaturen stattfindet (siehe Abschnitt 2.5.1). Der Ordnungs-UnordnungsÜbergang (ODT) kennzeichnet den Wechsel der homogenen, gemischten Phase in den phasenseparierten Zustand des Systems. Folglich kann bei einer Mischung des Systems eine zusätzliche Temperatur ( $\left.T_{\mathrm{ODT}}\right)$ ermittelt werden. Bei den in Tabelle 4-17 dargestellten Proben $\mathbf{V}$ bis IX wurde bei den DMA-Messungen kein zusätzlicher Übergang festgestellt, wobei anzumerken ist, dass die Untersuchungen ein Temperaturlimit von $T<350{ }^{\circ} \mathrm{C}$ besitzen, da ab dieser 
Temperatur die Zersetzung der Polymere beginnt. Bei der Probe $\mathbf{X}$ wurde für den P(MMA-co-CMA)-Block eine $T_{\mathrm{g}}$ von $140{ }^{\circ} \mathrm{C}$ bestimmt (siehe Abbildung 4-25), wobei diese Temperatur deutlich über dem literaturbekannten $T_{\mathrm{g}}$-Wert von PMMA liegt. Es ist zu erwähnen, dass für die Bestimmung der Glasübergangstemperaturen bei Probe $\mathbf{X}$ die Peaktemperatur von $\tan \delta$ verwendet wurde, wobei dies bei DMAMessungen eine durchaus bekannte Methode ist. Allerdings muss berücksichtigt werden, dass durch diese Bestimmungsmethode die $T_{\mathrm{g}}$ um 5 bis $10^{\circ} \mathrm{C}$ ansteigen kann. ${ }^{166}$ Jedoch ist der Wert der gemessenen $T_{\mathrm{g}}\left(140^{\circ} \mathrm{C}\right)$ auch nach Berücksichtigung des Fehlers zu hoch. Dementsprechend wird bei der ermittelten Temperatur von Probe $\mathbf{X}\left(T_{\mathrm{g}, 1}=140^{\circ} \mathrm{C}\right)$ von einer Überlagerung der $T_{\mathrm{g}} \operatorname{des} \mathrm{P}(\mathrm{MMA}-$ co-CMA)-Blocks und der $T_{\mathrm{ODT}}$ des Blockcopolymersystems ausgegangen. Die Existenz des ODTs bei höheren Temperaturen wurde für die Probe $\mathbf{X}$ mittels AFMUntersuchungen nachgewiesen (siehe Abschnitt 4.8).

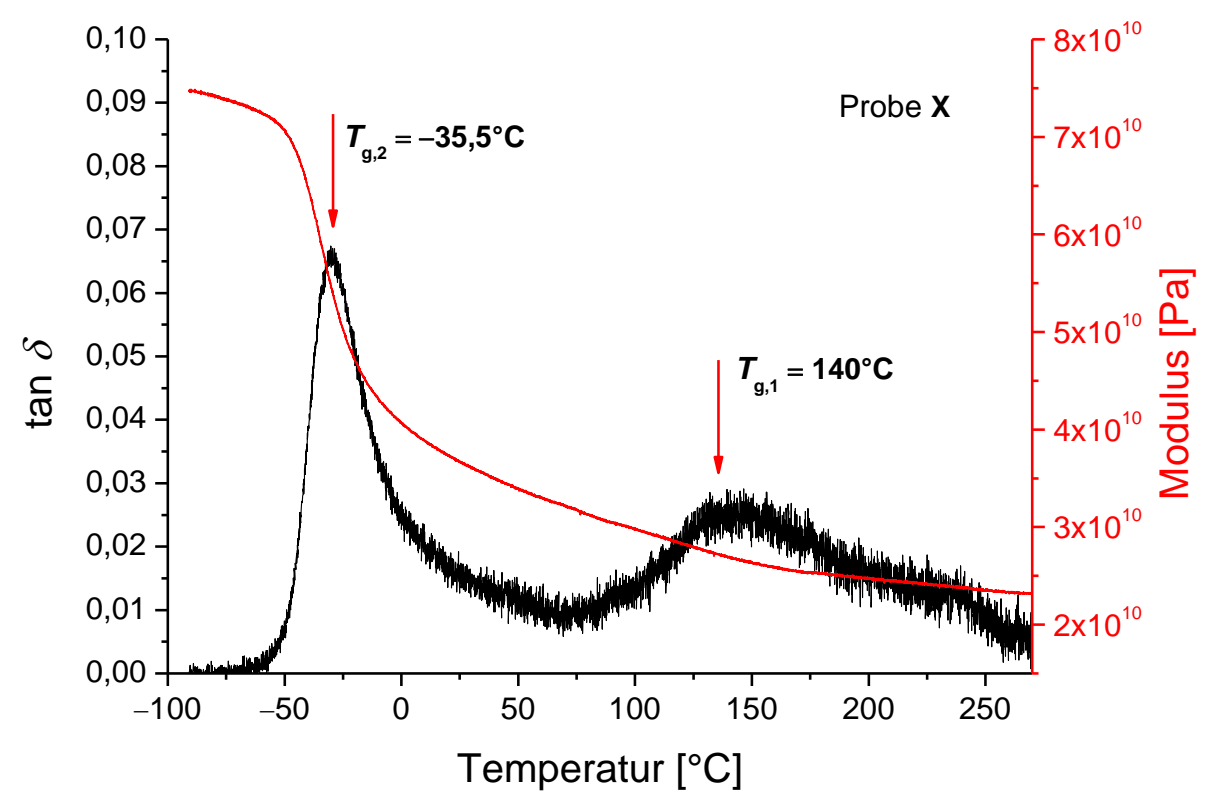

Abbildung 4-25 Darstellung der Glasübergangstemperaturen von Probe $\mathbf{X}$ in Abhängigkeit von dem temperaturabhängigen $\tan \delta$ und der Modulbestimmung. $T_{\mathrm{g}, 1}$ repräsentiert den P(MMA-coCMA)-Block und $T_{\mathrm{g}, 2}$ den PBA-Block.

Die ermittelten Glasübergangstemperaturen für das BCP P(MMA-co-CMA)- $b$-PBMA sind in der Tabelle 4-18 zusammengefasst, wobei $T_{\mathrm{g}, 1}$ den P(MMA-co-CMA)-Block repräsentiert und $T_{\mathrm{g}, 2}$ den PBMA-Block. Der Literaturwert für PBMA liegt bei $T_{\mathrm{g}} \approx 20^{\circ} \mathrm{C} .164$ 
Tabelle 4-18 Übersicht über Glasübergangstemperaturen der synthetisierten BCP P(MMA-coCMA)- $b$-PBMA mit den dazugehörigen Molmassen $\bar{M}_{\mathrm{n}}$, Dispersitäten $\doteq$ und molaren Anteilen $F_{\mathrm{BMA}}$.

\begin{tabular}{|l|l|l|l|l|l|}
\hline Probe & $\overline{\boldsymbol{M}}_{\mathbf{n}}[\mathbf{g} / \mathbf{m o l}]$ & $\boldsymbol{D}$ & $\begin{array}{l}\boldsymbol{F}_{\text {BMA }} \\
{[\mathbf{m o l} \%]}\end{array}$ & $\boldsymbol{T}_{\mathrm{g}, \mathbf{1}}\left[{ }^{\circ} \mathbf{C}\right]$ & $\boldsymbol{T}_{\mathrm{g}, 2}\left[{ }^{\circ} \mathbf{C}\right]$ \\
\hline XI & 40600 & 1,41 & 64 & $/$ & 54 \\
\hline XII & 100200 & 1,67 & 91 & 111 & 34 \\
\hline
\end{tabular}

Für Probe XII konnten zwei signifikante $T_{\mathrm{g}}$-Werte bestimmt werden, die auf eine MPS hindeuten. Bei der Probe XI wurde nur die $T_{\mathrm{g}, 2}$ für den PBMA-Block festgestellt, da der zweite Glasübergang $\left(T_{\mathrm{g}, 1}\right)$ sehr breit war und nicht ausgewertet werden konnte. Trotz der Ergebnisse der thermischen Untersuchung für Probe XI zeigen die AFM-Höhenprofile ein phasensepariertes System (siehe Abschnitt 4.4.2). 


\subsection{Bestimmung der Mikrophasenseparation der Blockcopolymersysteme mittels AFM}

Die Rasterkraftmikroskopie (AFM, siehe Abschnitt 2.6) bietet eine gute Möglichkeit die Höhenprofile der Probenoberfläche zu ermitteln und zusätzlich Informationen über die mechanischen Eigenschaften $\mathrm{zu}$ erhalten. Die Untersuchung der synthetisierten Blockcopolymere (BCP) mit Hilfe des AFMs eignet sich hervorragend um detaillierte Informationen über die Mikrophasenseparation (MPS) der jeweiligen Polymersysteme zu erhalten.

\subsubsection{Untersuchung des Blockcopolymersystems P(MMA-co-CMA)- $b$-PBA}

In diesem Abschnitt erfolgt die AFM-Untersuchung der Oberfläche des BCPSystems P(MMA-co-CMA)- $b$-PBA, sowie der Oberfläche des Polymersystems ohne Coumarin-Einheiten (PMMA- $b$-PBA). Die Orientierung der jeweiligen Morphologie des BCPs ist von der Oberflächenenergie der einzelnen Blöcke abhängig. Aufgrund der niedrigeren Oberflächenenergie des PBA-Blocks $(31 \mathrm{mN} / \mathrm{m})$ im Vergleich zum PMMA-Block $(41 \mathrm{mN} / \mathrm{m})$ neigt dieser zur Entmischung an der freien Oberfläche. ${ }^{167-170}$ Der polare Ausgangsblock (PMMA) bevorzugt die Anordnung auf dem polaren Glas-Substrat, wodurch die Orientierung der jeweiligen Blöcke parallel zum Substrat erfolgt [siehe Schema 4-1 a)]. Durch diesen Effekt wäre die Untersuchung der MPS an der Probenoberfläche nicht durchführbar und folglich ist eine Vorbehandlung der Substratoberfläche von entscheidender Bedeutung. Ein übliches Vorgehen um eine chemische Neutralität des Substrats zu erreichen, ist die Behandlung der Oberfläche mit einem statistischen Copolymer-Film, der die gleichen Monomereinheiten wie das BCP enthält. ${ }^{171-173}$ Dadurch wurde am Anfang ein dünner PMMA-co-PBA-co-PHEMA-Film (Zusammensetzung siehe Anhang A, Tabelle A-7) per spincoating auf das Glassubstrat aufgetragen und im Anschluss im Vakuumofen getempert, um eine kovalente Bindung zwischen den OH-Gruppen der HEMA-Einheiten im Copolymer und dem Glassubstrat $\left(\mathrm{SiO}_{2}\right) \mathrm{zu}$ erzeugen. Im zweiten Schritt wurde das BCP per spincoating auf das Substrat aufgetragen und ebenfalls im Vakuumofen getempert, bis sich das Gleichgewicht der Morphologie eingestellt hat. Schema 4-1 soll dieses Prinzip veranschaulichen. Die detaillierte Probenpräparation ist dem Abschnitt 6.6.3 zu entnehmen. 


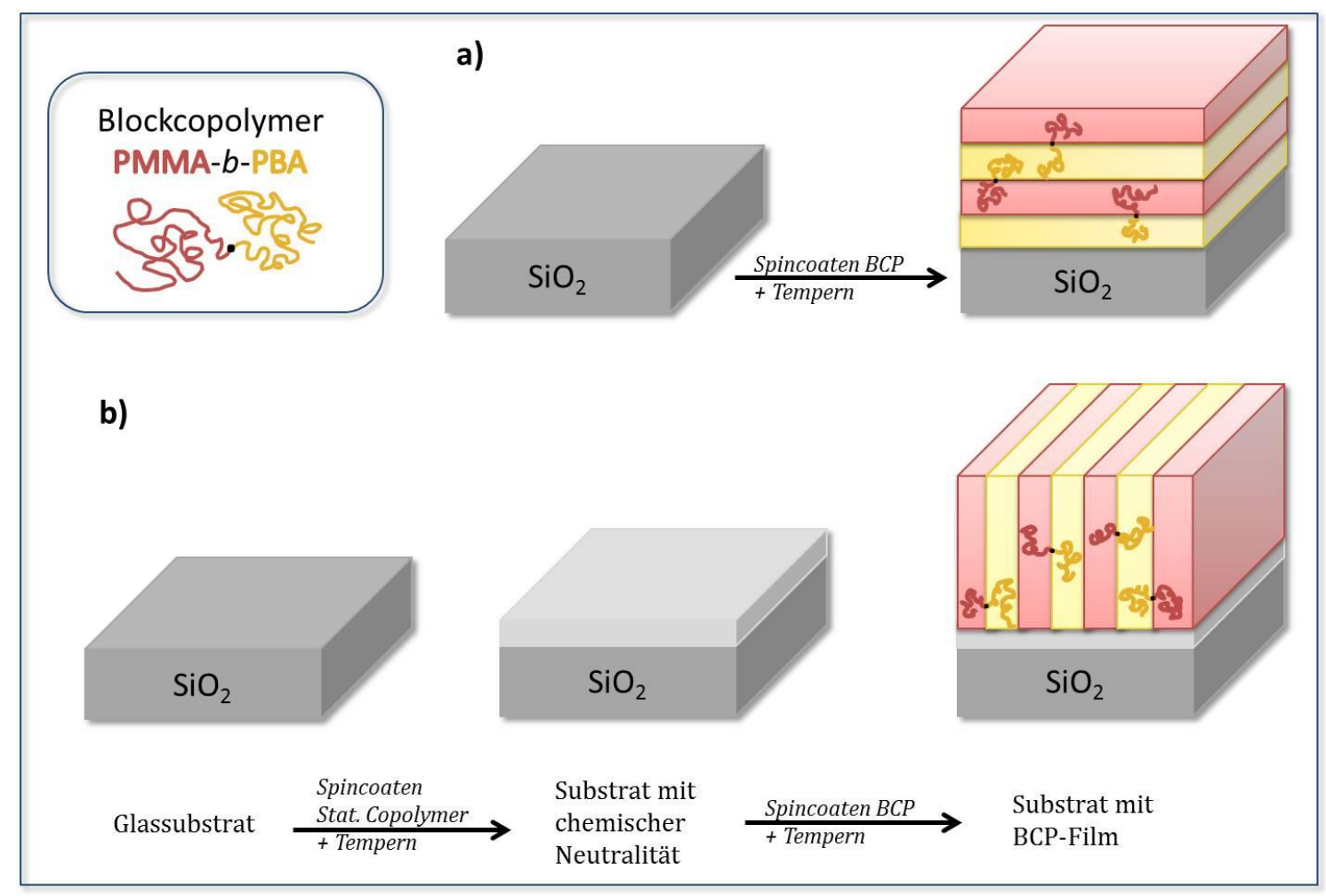

Schema 4-1 Übersicht über Probenpräparation für AFM-Messungen: a) ohne Vorbehandlung der Substratoberfläche; b) mit Vorbehandlung der Substratoberfläche.

Durch die Vorbehandlung des Substrats erfolgte eine senkrecht zum Substrat ausgerichtete Orientierung der einzelnen BCP-Domänen. Die verschiedenen Proben des BCP-Systems P(MMA-co-CMA)- $b$-PBA (IX, VII, VI, V) wurden mit dem AFM untersucht und die AFM-Höhenprofile für die unterschiedlichen Polymeroberflächen sind in Abbildung 4-26 dargestellt. Für ein Verständnis der in der vorliegenden Arbeit befindlichen AFM-Höhenprofile ist anzumerken, dass die für die AFM-Bilder gewählte Farbskala die tiefer gelegenen Objekte dunkel, rotbraun kennzeichnet und die höher gelegenen Bereiche hell, gelb darstellt. Für die Messung der folgenden Höhenprofile wurde die Krafteinwirkung auf die Probenoberfläche (PF setpoint) automatisch vom AFM-Messprogramm gesetzt. Dadurch wird zum einen die Cantileverspitze geschont und zum anderen wird die Probenoberfläche nicht beeinflusst. 

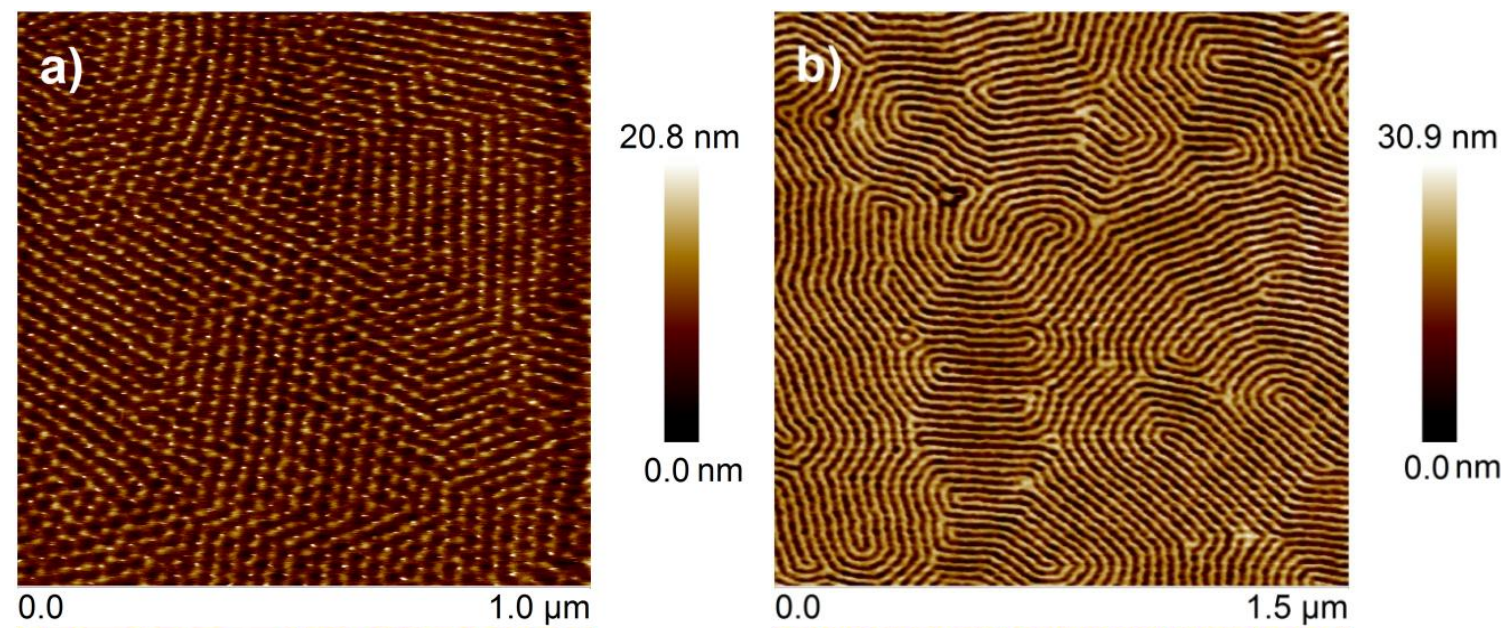

$1.5 \mu \mathrm{m}$
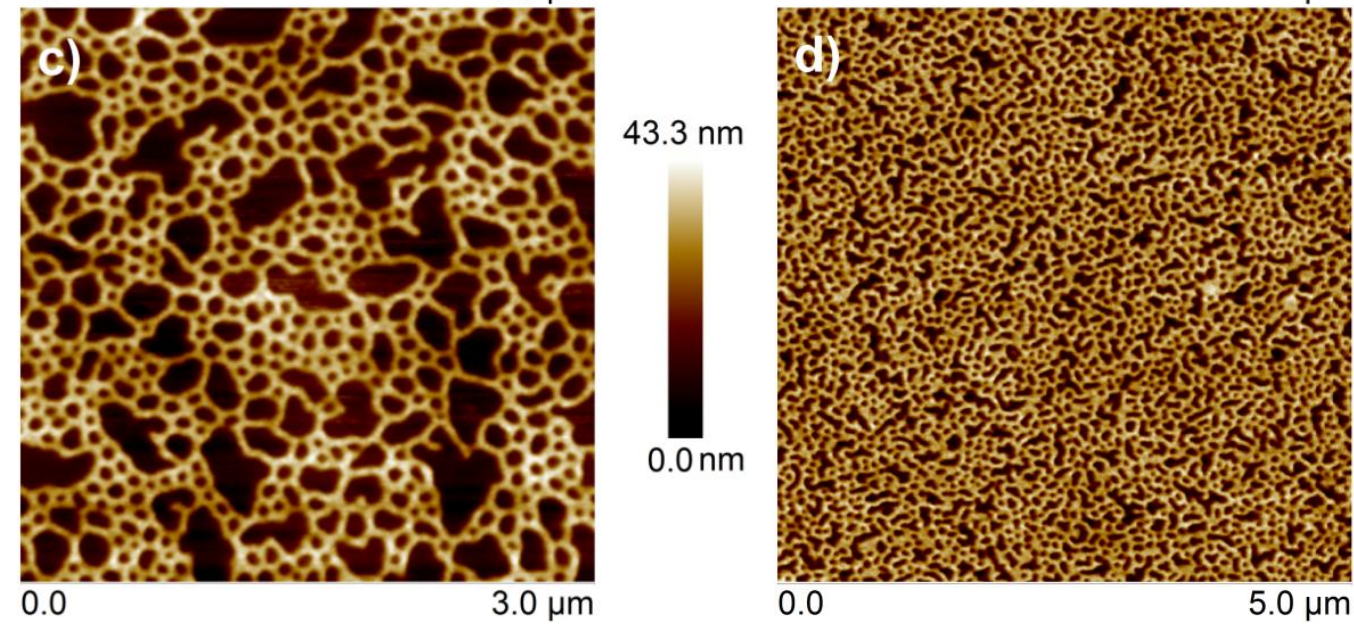

$33.6 \mathrm{~nm}$

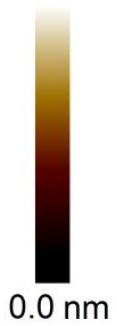

Abbildung 4-26 Höhenprofile von P(MMA-co-CMA)- $b$-PBA mit unterschiedlichen Zusammensetzungen: a) Probe IX mit $F_{\mathrm{BA}}=79 \mathrm{~mol} \%$; b) VII mit $F_{\mathrm{BA}}=68 \mathrm{~mol} \%$; c) VI mit $F_{\mathrm{BA}}=61 \mathrm{~mol} \%$; d) V mit $F_{\mathrm{BA}}=52 \mathrm{~mol} \%$; Polymerfilme wurden bei $160^{\circ} \mathrm{C}$ über Nacht getempert. Cantilever: FESPA. PF setpoint automatisch gesetzt. Auflösung jew. 512 x 512 Pixel.

Die AFM-Höhenprofile in Abbildung 4-26 stellen unterschiedliche, phasenseparierte Strukturen für das Blockcopolymersystem P(MMA-co-CMA)- $b$-PBA dar, wobei eine Abhängigkeit der MPS von der Zusammensetzung des Blockcopolymers festzustellen ist. Die Phasenseparation bei Probe IX [Abbildung 4-26 a)] besteht aus einer Anordnung runder Objekte, eingebettet in einem dunklen Hintergrund, die sich zu Domänen mit Domänengrenzen anordnen. Aus der lokalen Charakterisierung der mechanischen Eigenschaften der DMT-Modul-Profile (siehe Abschnitt 4.6) folgt, dass es sich um P(MMA-co-CMA)-Sphären handelt, die sich in einer PBA-Matrix (dunkle Phase) befinden. Der mittlere Domänenabstand $D$ der regelmäßig angeordneten Sphären kann mit Hilfe der PSD-Funktion ermittelt werden (siehe Abschnitt 2.7.4). Die sich wiederholenden Strukturgrößen in der PSD-Verteilung demonstrieren hierbei die Sphären (Abbildung 4-27), wobei das Maximum des Peaks in der Verteilung den Domänenabstand der Sphären darstellt. 
Der ermittelte Durchschnittsabstand zwischen den Sphärenzentren liegt bei $33,7 \mathrm{~nm}\left(f=29,7 \mu \mathrm{m}^{-1}\right)$.
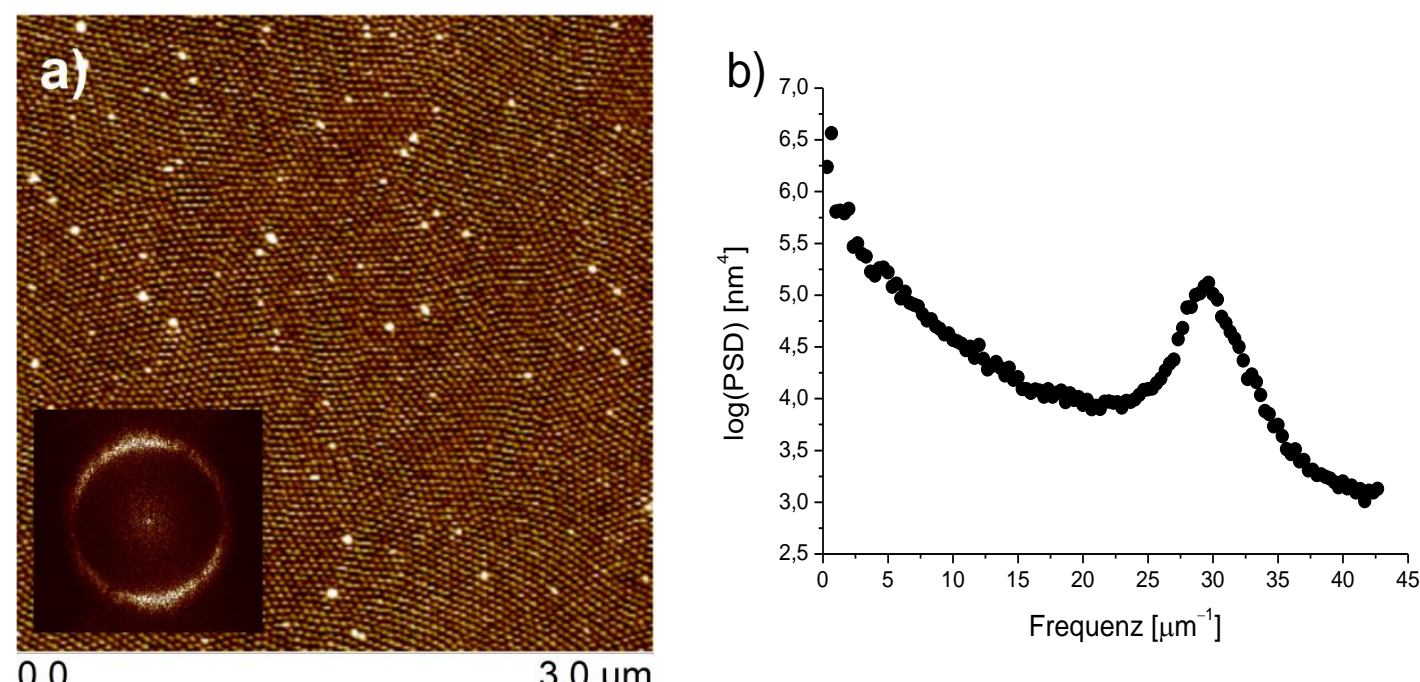

Abbildung 4-27 Bestimmung des Domänenabstands mittels PSD-Funktion: a) AFM-Höhenprofil von Probe IX, wobei der Ausschnitt die FFT zeigt. Cantilever: FESPA. PF setpoint automatisch gesetzt. Auflösung jew. $512 \times 512$ Pixel. b) zweidimensionale, isotropische PSD-Verteilung des Höhenprofils.

Die in Abschnitt 4.2.3 aufgeführten Blockcopolymere VIII und IX besitzen eine ähnliche Polymerzusammensetzung ( $F_{\mathrm{BA}}$, siehe Tabelle 4-15) und die daraus resultierende Strukturähnlichkeit der beiden Polymere konnte mit den AFMUntersuchungen nachgewiesen werden. Für die im Höhenprofil der Probe VIII (Anhang B, Abbildung B-1) veranschaulichten Sphären wurde ein Domänenabstand von $32,6 \mathrm{~nm}$ bestimmt.

Die Probe VII in Abbildung 4-26 b) zeigt, dass der zunehmende P(MMA-co-CMA)Anteil $\left(F_{\mathrm{BA}}=68 \mathrm{~mol} \%\right) \mathrm{zu}$ einer Änderung in der Morphologie führt (siehe Tabelle 4-19). Die höher gelegenen Objekte sind P(MMA-co-CMA)-Zylinder, die parallel zur Oberfläche in einer PBA-Matrix (dunkle Phase) liegen. Die Zylinder erscheinen in einer mäanderförmigen Struktur mit verschiedenen Kontaktpunkten und Biegungswechseln. Diese bemerkenswerte Struktur wird in Abschnitt 4.5 näher erläutert. Der Domänenabstand der Zylinder wurde auch hier mittels der PSDFunktion bestimmt und die PSD-Verteilung ist in Abbildung 4-28 dargestellt. Die sich wiederholende Strukturgröße der Domänen liegt bei 31,8 nm $\left(f=31,4 \mu \mathrm{m}^{-1}\right)$. Obwohl sich die drei BCP VII, VIII und IX teilweise in ihrer Morphologie unterscheiden, wurde für die drei P(MMA-co-CMA)-Blöcke (Sphären und Zylinder) der Polymerproben ein ähnlicher Domänenabstand bestimmt (siehe Tabelle 4-19). 
Dieses Ergebnis war zu erwarten, da das makroRAFT-Agens $\left(\bar{M}_{\mathrm{n}}=12000 \mathrm{~g} / \mathrm{mol}\right.$, siehe Tabelle 4-15) für die BCP VII, VIII und IX identisch ist. Im Gegensatz zu diesem Ergebnis wurde bei der Probe $\mathbf{X}$ für den kürzeren P(MMA-co-CMA)-Block $\left(\bar{M}_{\mathrm{n}}=5800 \mathrm{~g} / \mathrm{mol}\right.$, siehe Tabelle 4-15) ein kleinerer Domänenabstand von ca. 19,5 nm ermittelt [PSD-Verteilung siehe Abbildung B-2 b), Anhang B]. Das Blockcopolymer $\mathbf{X}\left(F_{\mathrm{BA}}=69 \mathrm{~mol} \%\right)$ besitzt die gleiche Polymerzusammensetzung wie Probe VII $\left(F_{\mathrm{BA}}=68 \mathrm{~mol} \%\right.$, siehe Tabelle 4-15) und das AFM-Höhenprofil (siehe Abbildung B-2 a), Anhang B) zeigt wie bei Probe VII höher gelegene P(MMA-coCMA)-Zylinder, die in einer PBA-Matrix eingebettet sind.
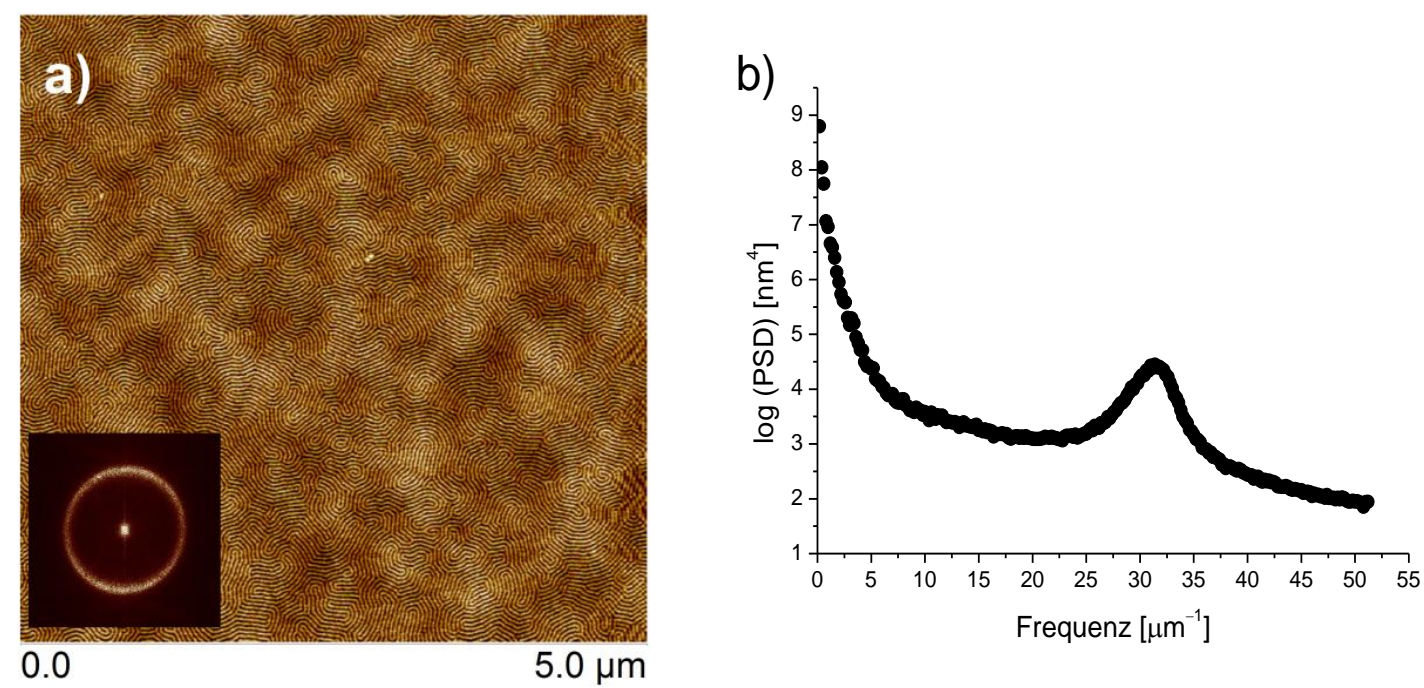

Abbildung 4-28 Bestimmung des Domänenabstands mittels PSD-Funktion: a) AFM-Höhenprofil von Probe VII, wobei der Ausschnitt die FFT zeigt. Cantilever: FESPA. PF setpoint automatisch gesetzt. Auflösung jew. $512 \times 512$ Pixel. b) zweidimensionale, isotropische PSD-Verteilung des Höhenprofils.

Bei der Betrachtung der Probenoberflächen von V und VI [Abbildung 4-26 c) und d)] wird eine Insel-Loch-Morphologie sichtbar. Die höher gelegenen P(MMA-coCMA)-Domänen stellen perforierte Lamellen dar, die horizontal zum Substrat orientiert sind und in einer darunterliegenden PBA-Phase eingebettet sind. Bei Betrachtung der Blockcopolymerdimension von Probe V und VI fällt auf, dass die PBA-Gebiete in beiden Fällen größer als erwartet sind. Es wird davon ausgegangen, dass es sich bei diesen Phasenbereichen um parallel zur Oberfläche orientierte PBA-Lamellen handelt, in denen die Blöcke wahrscheinlich zum größten Teil senkrecht zur Oberflächenebene ausgerichtet sind.

In dem AFM-Höhenprofil von Probe V [Abbildung 4-26 d)] erscheint die PMMADomäne im Vergleich zur Domäne von Probe VI [Abbildung 4-26c)] mit 
zunehmenden molaren Anteil des makroRAFT-Agens ( $\left.F_{\mathrm{MMA-co-CMA}}\right)$ größer. Die starke Streuung bei der durchgeführten FFT spiegelt die große Schwankung bei den Werten der Lamellenabstände wider. Deshalb zeigt die PSD-Verteilung beider Proben einen breit verteilten Peak, wobei das mittlere Maximum für Probe VI bei ca. 85,7 nm liegt und für Probe $\mathbf{V}$ bei ca. 86,2 nm. Da das makroRAFT-Agens für die BCP V und VI identisch ist (siehe Tabelle 4-15), war eine Übereinstimmung der Domänenabstände beider Proben zu erwarten.

Der Domänenabstand der perforierten Lamellen liegt für Probe VI bei ca. 85,7 nm und für Probe $\mathbf{V}$ bei ca. $86,2 \mathrm{~nm}$, wobei die PSD-Verteilungen der beiden Höhenprofile dem Anhang B (Abbildung B-3 und Abbildung B-4) zu entnehmen sind. Da das makroRAFT-Agens für die BCP V und VI identisch ist (siehe Tabelle 4-8), war wieder eine Übereinstimmung der Domänenabstände beider Proben zu erwarten. Tabelle 4-19 fasst nochmal die Zusammensetzungen der unterschiedlichen BCP, sowie die ermittelten Domänenabstände der P(MMA-co-CMA)Phase zusammen.

Tabelle 4-19 Morphologie der synthetisierten BCP [P(MMA-co-CMA)- $b$-PBA].

\begin{tabular}{|l|l|l|l|}
\hline Probe & $\boldsymbol{F}_{\text {BA }}[\mathbf{m o l} \%]$ & $\boldsymbol{D}[\mathbf{n m}]$ & Morphologie \\
\hline V & 52 & 86,2 & Parallel perforierte Lamellen \\
\hline VI & 61 & 85,7 & Parallel perforierte Lamellen \\
\hline VII & 68 & 31,8 & Zylinder \\
\hline VIII & 75 & 32,6 & Sphären \\
\hline IX & 79 & 33,7 & Sphären \\
\hline $\mathbf{X}$ & 69 & 19,5 & Zylinder \\
\hline
\end{tabular}

\section{Vergleich der Blockcopolymersysteme P(MMA-co-CMA)-b-PBA und PMMA-b- PBA}

Unabhängig von den in Abschnitt 4.2 .1 beschriebenen Blockcopolymerisationen wurde ein zusätzliches Polymersystem ohne Coumarin-Einheiten (PMMA- $b$-PBA) hergestellt, wobei die Reaktionsbedingungen für die Herstellung des Systems der Tabelle A-13 in Anhang A zu entnehmen sind. Dieses BCP dient als Referenzsystem um einen Einfluss der Coumarin-Einheiten auf die MPS des Systems ausschließen zu können. In diesem kurzen Unterkapitel soll ein Vergleich der AFMUntersuchungen der phasenseparierten Systeme mit und ohne UV-vernetzende Einheiten erfolgen. In Abbildung 4-29 sind AFM-Höhenprofile von dem BCP 
PMMA- $b$-PBA, das sich jeweils in seiner Molmasse sowie Zusammensetzung (Tabelle 4-20) unterscheidet, dargestellt.
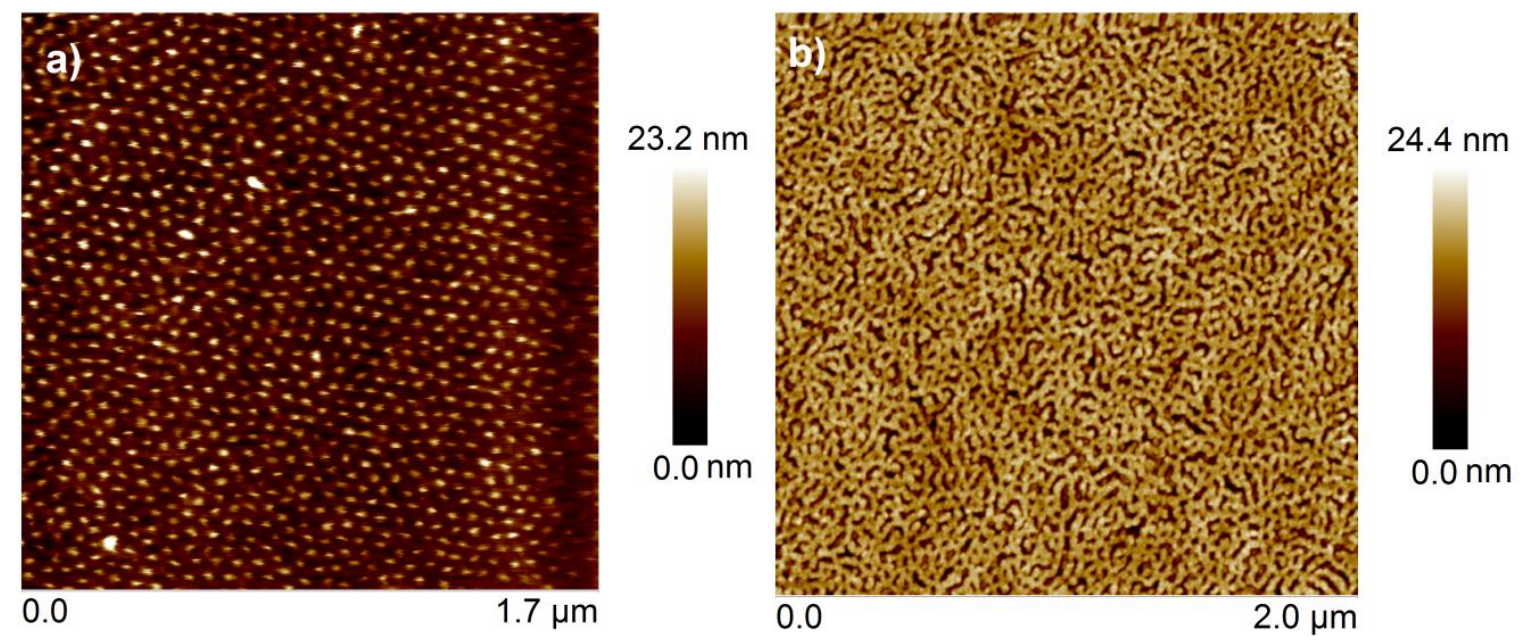

Abbildung 4-29 AFM-Höhenprofile für das BCP PMMA- $b$-PBA: a) mit $F_{\mathrm{BA}}=85 \mathrm{~mol} \%$ und $\bar{M}_{\mathrm{n}}=78300 \mathrm{~g} / \mathrm{mol} ; \quad$ b) mit $F_{\mathrm{BA}}=55 \mathrm{~mol} \%$ und $\bar{M}_{\mathrm{n}}=43000 \mathrm{~g} / \mathrm{mol}$. Cantilever: ScanAsystAir. PF setpoint automatisch gesetzt. Auflösung jew. 512 x 512 Pixel.

Tabelle 4-20 Übersicht für die BCP PMMA- $b$-PBA aus Abbildung 4-29 mit molaren Anteil von BA im BCP, $F_{\mathrm{BA}}$, der Molmasse und Dispersität des makroRAFT-Agens PMMA, $\bar{M}_{\mathrm{n}, \mathrm{PMMA}}$ und $\bigoplus_{\mathrm{PMMA}}$ sowie Molmasse und Dispersität des BCP, $\bar{M}_{\mathrm{n}, \mathrm{PMMA}-b-\mathrm{PBA}}$ und $\bigoplus_{\mathrm{PMMA}-b \text {-PBA}}$.

\begin{tabular}{|l|l|l|l|l|l|l|}
\hline Probe & $\begin{array}{l}\overline{\boldsymbol{M}}_{\text {n,PMMA }} \\
\text { [g/mol] }\end{array}$ & $\boldsymbol{Ð}_{\text {PMMA }}$ & $\begin{array}{l}\overline{\boldsymbol{M}}_{\text {n,PMMA-b-PBA }} \\
\text { [g/mol] }\end{array}$ & $\begin{array}{l}\boldsymbol{Ð}_{\text {PMMA-b- }} \\
\text { PBA }\end{array}$ & $\begin{array}{l}\boldsymbol{F}_{\text {BA }} \\
\text { [mol\%] }\end{array}$ & $\begin{array}{l}\text { Anzahl } \\
\text { der BA- } \\
\text { Einheiten }\end{array}$ \\
\hline $\begin{array}{l}\text { PMMA }_{95}-b- \\
\text { PBA }_{537}\end{array}$ & 11900 & 1,32 & 78300 & 1,22 & 85 & 537 \\
\hline $\begin{array}{l}\text { PMMA }_{167}-b- \\
\text { PBA }_{205}\end{array}$ & 20900 & 1,36 & 43000 & 1,33 & 55 & 205 \\
\hline
\end{tabular}

Das BCP PMMA95- $b-\mathrm{PBA}_{537}$ in Abbildung 4-29 a) zeigt die für den molaren Anteil von $\mathrm{BA}\left(F_{\mathrm{BA}}=85 \mathrm{~mol} \%\right)$ typische Sphärenstruktur, wobei die PMMA-Sphären in einer darunterliegen PBA-Matrix eingebettet sind. Bei dem BCP mit dem geringeren BA-Anteil $\left(F_{\mathrm{BA}}=55 \mathrm{~mol} \%\right)$ ordnet sich die PMMA-Phase zu horizontal orientierten perforierten Lamellen an, die ebenfalls in einer PBA-Matrix eingebettet sind [Abbildung 4-29 b)]. Insgesamt zeigen die BCP ohne CoumarinEinheiten (Abbildung 4-29) eine ähnliche MPS wie die Polymersysteme mit photovernetzbaren Coumarin aus dem vorangegangenen Abschnitt. Der Einbau kleiner Mengen von Vernetzer-Anteilen wirkt sich somit nicht auf die MPS aus, lediglich 
die Zusammensetzung der Blockkomponenten im System beeinflusst bei einer konstant gehaltenen Temperatur die MPS des Blockcopolymers.

\section{Untersuchung der Mikrophasenseparation bei erhöhten Temperaturen}

Das Phasenverhalten von Blockcopolymeren ist stark temperaturabhängig, da mit steigender Temperatur die Mischbarkeit der einzelnen Blöcke zunimmt (siehe Abschnitt 2.5.1) und ab der kritischen Temperatur $T_{\text {ODT }}$ erfolgt der Übergang in eine gemischte, homogene Phase. Folglich wurde das Phasenverhalten des BCPs P(MMA-co-CMA)- $b$-PBA mit Hilfe des AFMs bei erhöhten Temperaturen untersucht. Für diese Messungen wurde das AFM mit einer Heizzelle ausgestattet, die die Proben auf Temperaturen bis zu ca. $250{ }^{\circ} \mathrm{C}$ aufheizen kann. Die folgenden Untersuchungen wurden mit dem BCP VII (siehe Tabelle 4-15) durchgeführt. Für eine bessere Unterscheidung der einzelnen AFM-Proben wird eine zusätzliche Nummerierung eingeführt (siehe Tabelle 4-21), wobei die Probe VII (1) zweimal hintereinander aufgeheizt wurde (VII (1a) und VII (1) )).

Tabelle 4-21 Übersicht über BCP $\mathrm{P}\left(\mathrm{MMA}_{95}-c o-\mathrm{CMA}_{9}\right)-b-\mathrm{PBA}_{229}$ (VII) für verschiedene AufheizZyklen.

\begin{tabular}{|l|l|}
\hline Probe & Aufheiz-Zyklen \\
\hline VII (1a) & $25^{\circ} \mathrm{C}-180^{\circ} \mathrm{C}$ \\
\hline VII (1b) & $25^{\circ} \mathrm{C}-210^{\circ} \mathrm{C}$ \\
\hline VII (2) & $25^{\circ} \mathrm{C}-160^{\circ} \mathrm{C}$ \\
\hline
\end{tabular}

Die Probe VII wurde zuerst zwei Aufheiz-Zyklen unterzogen. Der erste Zyklus wurde in einem Temperaturbereich von 25 bis $180^{\circ} \mathrm{C}$ durchgeführt und der zweite Zyklus von 25 bis $210^{\circ} \mathrm{C}$. In Abbildung 4-30 sind Ausschnitte der AFM-Höhenprofile der Probe VII (1a) bei den jeweiligen Temperaturen des ersten AufheizZyklus dargestellt. Das Höhenprofil bei $25^{\circ} \mathrm{C}$ (Abbildung 4-30 a)) zeigt die bereits in Abbildung 4-26 b) vorgestellte Zylinderstruktur. Die Messungen bei hohen Temperaturen bringen einige Messprobleme mit sich (siehe Abschnitt 6.6.3). Bei den Temperaturen $70{ }^{\circ} \mathrm{C}$ und $130{ }^{\circ} \mathrm{C}$ (Abbildung 4-30 b) und c)) kommt es an bestimmten Stellen der Struktur (Defekte, siehe Abschnitt 4.5.1) zur Schädigung der Probenoberfläche (tiefe „Löcher“, beispielhaft gekennzeichnet durch weißen Rahmen in Abbildung 4-30). Durch eine zu groß gewählte Krafteinstellung für den Cantilever (PF setpoint) kann die Probenoberfläche, besonders bei hohen Temperaturen, beschädigt werden. Für die Probe VII (1) wurde für beide AufheizZyklen der PF setpoint von dem AFM-Messprogramm automatisch gesetzt, wobei 
diese Kraft für die Polymeroberfläche $\mathrm{zu}$ hoch ist. Mit zunehmender Kettenbeweglichkeit $\left(T>180^{\circ} \mathrm{C}\right)$ kann das System ausheilen und die „Löcher" verschwinden. Allerdings muss dem Material genügend Zeit für den Ausheilprozess gegeben werden, da sich sonst die Gleichgewichtsmorphologie nicht einstellen kann und die Strukturschädigung („Löcher“) nach dem Abkühlen noch vorhanden ist (Ausgangsprobe für zweiten Aufheiz-Zyklus; siehe weißer Rahmen in Abbildung 4-31 a)). Bei den Abbildung 4-31 e) und f) musste der polynome Hintergrund rechnerisch aus den AFM-Profilen entfernt werden, sodass die Oberflächenstruktur der MPS besser zu sehen ist.
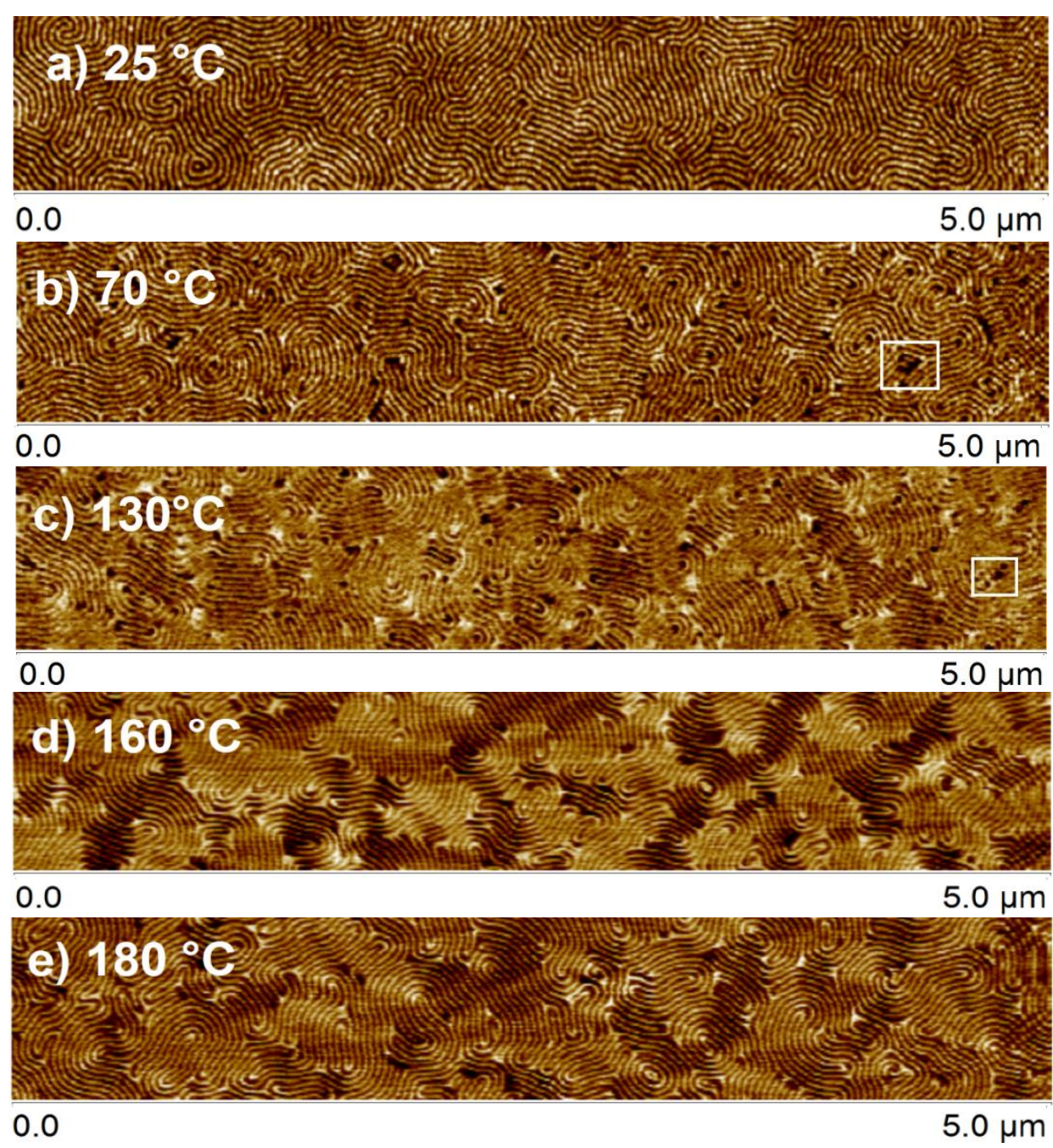

Abbildung 4-30 AFM-Höhenprofile von Probe VII (1a) für den ersten Aufheiz-Zyklus von 25 bis $180^{\circ} \mathrm{C}$. Der weiße Rahmen in b) und c) kennzeichnet beispielhaft die „Löcher“. PF setpoint automatisch gesetzt. Cantilever: ScanAsystAir. 

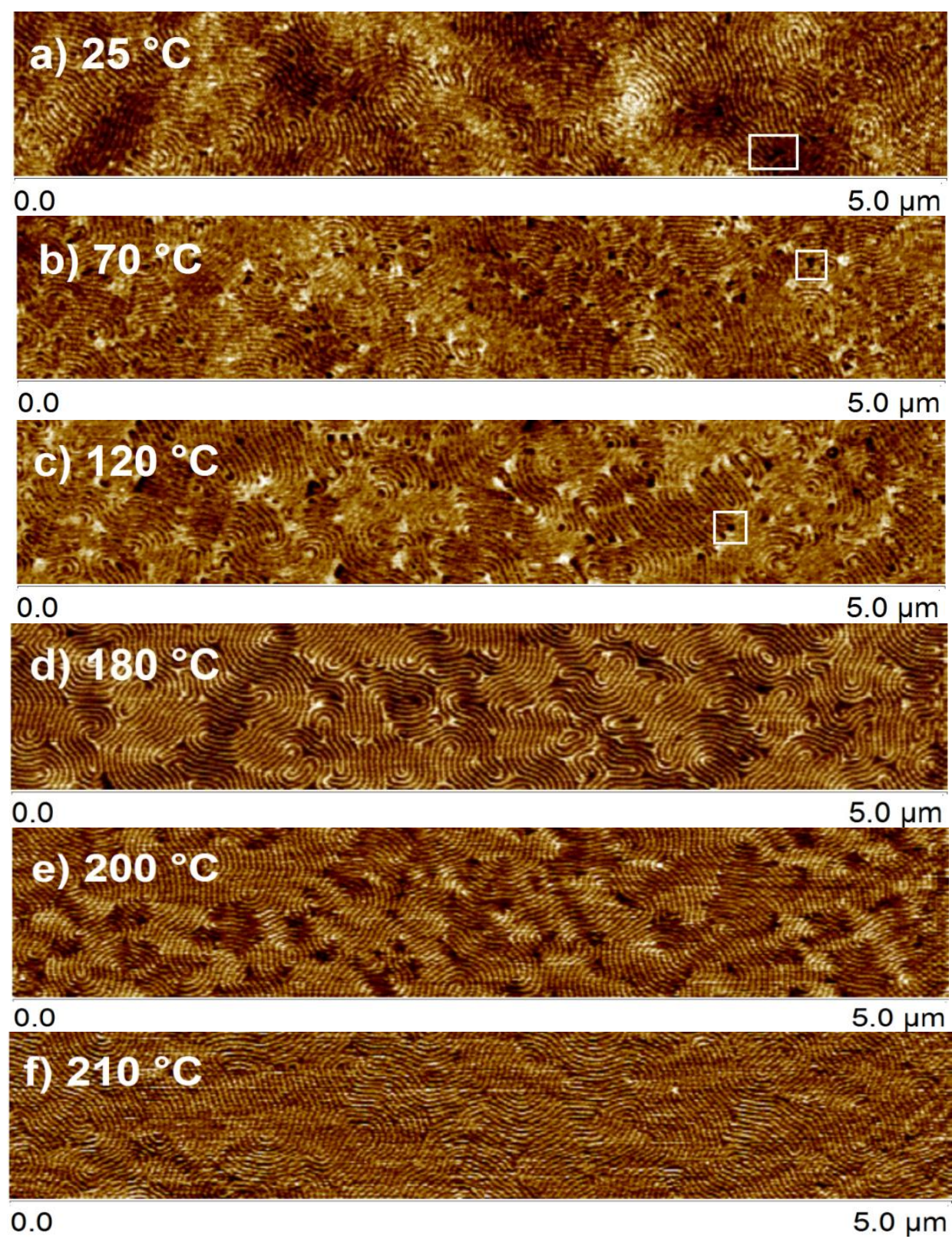

Abbildung 4-31 AFM-Höhenprofile von Probe VII (1b) für den zweiten Aufheiz-Zyklus von 25 bis $210^{\circ} \mathrm{C}$. Der weiße Rahmen in a), b) und c) kennzeichnet beispielhaft die „Löcher“. PF setpoint automatisch gesetzt. Cantilever: ScanAsystAir.

Zur Vermeidung der Strukturschädigung durch den Cantilever wird eine weitere AFM-Probe [VII(2)] mit einem konstant niedrigen PF setpoint untersucht. Die Untersuchung der Probe VII (2) erfolgt in einem Temperaturbereich von 25 bis $160{ }^{\circ} \mathrm{C}$ (Abbildung 4-32). Bei der Messung der Probe VII (2) wird deutlich, dass über den gesamten Temperaturbereich keine Schädigung der Probenoberfläche auftritt. Das Höhenprofil zeigt nach dem Abkühlen (Abbildung 4-32 e)) dieselbe klare Strukturdefinition wie die Ausgangsprobe bei $25^{\circ} \mathrm{C}$ [Abbildung 4-32 a)]. Der 
kontrollierte $P F$ setpoint ist für die Messungen optimal gewählt, allerdings konnten mit diesem Wert nicht so hohe Temperaturen wie in Abbildung 4-31 erreicht werden. Grund für dieses Messproblem könnte das thermische Rauschen des Cantilevers (siehe Abschnitt 6.6.3) bei hohen Temperaturen sein. Um diesen Effekt $\mathrm{zu}$ überprüfen, wurden ähnliche Messungen mit dem RFESP-Cantilever durchgeführt, da dieser im Vergleich zum ScanAsystAir-Cantilever eine größere Federkonstante $k_{c}$ besitzt ( $3 \mathrm{~N} / \mathrm{m}$ vs. $0,4 \mathrm{~N} / \mathrm{m}$ ). Die Verringerung des thermischen Rauschens bei höheren Temperaturen kann zwar festgestellt werden, allerdings können weiterhin keine Messungen bei Temperaturen über $200^{\circ} \mathrm{C}$ durchgeführt werden. Daraus lässt sich schließen, dass ein wesentlich größeres Problem die Messungen behindert. Mit steigender Temperatur nimmt die Klebrigkeit des gewählten Blockcopolymersystems zu und aufgrund zu hoher Adhäsion kann sich der Cantilever nicht von der Probe zurückziehen, wodurch eine Messung nicht mehr vorgenommen werden kann.

Zusätzlich wurden die Domänenabstände der Zylinder in Abhängigkeit von der Temperatur bestimmt. Dafür wurden die Höhenprofile mit einem Ausschnitt von $5 \mu \mathrm{m} \times 5 \mu \mathrm{m}$ mittels PSD-Funktion bei unterschiedlichen Temperaturen ausgewertet. Abbildung 4-33 zeigt die grafische Auftragung der Domänenabstände in Abhängigkeit von der Temperatur.

Der Domänenabstand $D$ für die Proben VII (1a) und VII (1b) nimmt anfangs bei beiden Aufheiz-Zyklen einen relativ konstanten Wert von ca. $33 \mathrm{~nm}$ an. Ab einer Temperatur von ca. $140{ }^{\circ} \mathrm{C}$ verkleinert sich der Zylinderabstand auf ca. $30 \mathrm{~nm}$. Bei der zweiten Probe VII (2) kann für den gewählten Temperaturbereich kein signifikantes Abfallen des Domänenabstandes festgestellt werden. Die Werte schwanken zwischen 34-35 nm und aufgrund von Messproblemen konnte die Probe bei höheren Temperaturen nicht untersucht werden. Die Verringerung des Domänenabstands bei Probe VII (1a) und VII (1b) könnte auf den Beginn des Ordnungs-Unordnungs-Übergangs zurückgeführt werden.174,175 Allerdings konnten ab $220^{\circ} \mathrm{C}$ keine weiteren Temperaturuntersuchungen mittels AFM durchgeführt werden und ein vollständiges Auflösen der Zylinderstrukturen für die Probe VII konnte bis zu dieser Temperatur nicht festgestellt werden. 


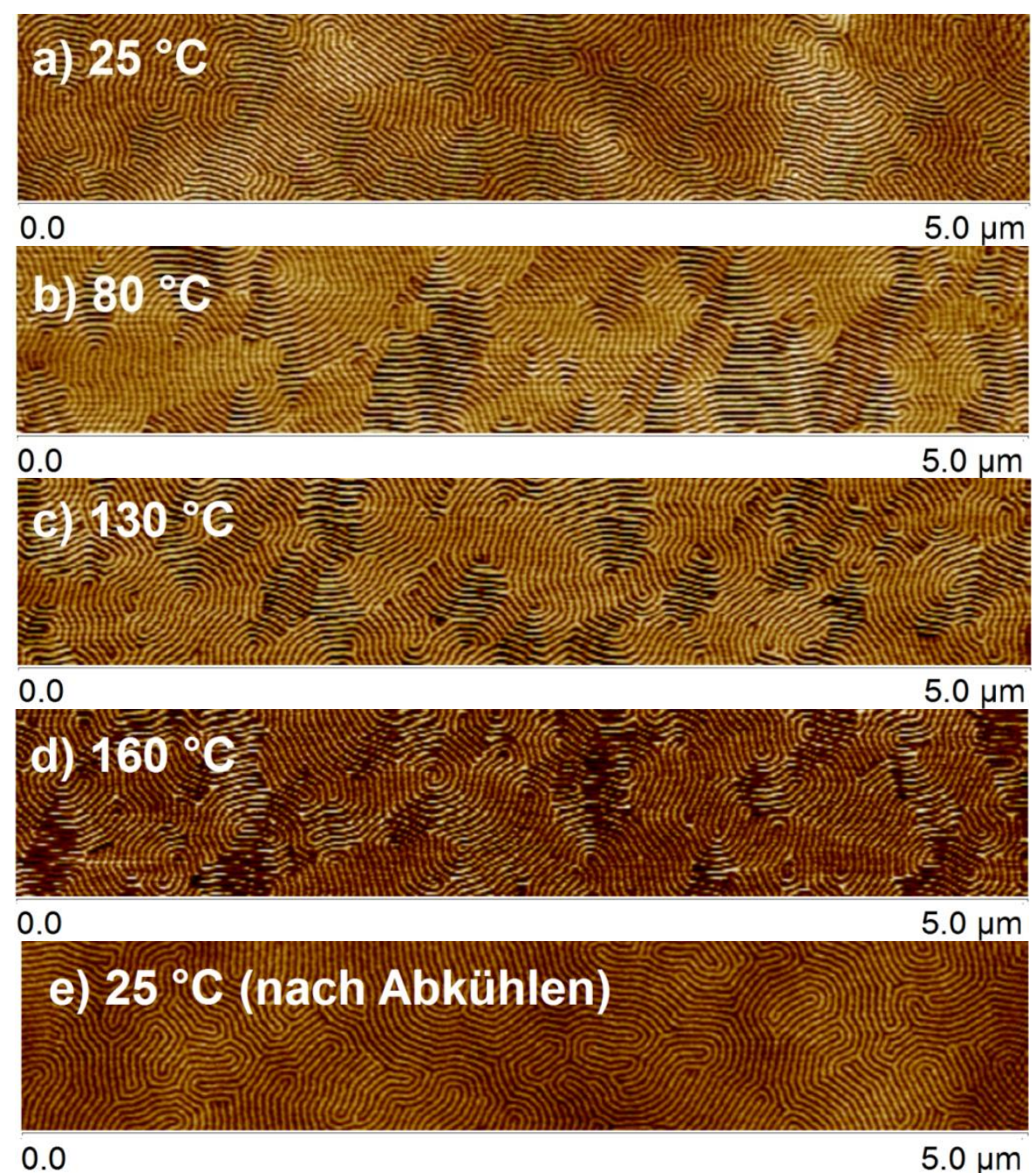

Abbildung 4-32 AFM-Höhenprofile von Probe VII (2) für einen Aufheiz-Zyklus von 25 bis $160^{\circ} \mathrm{C}$. PF setpoint von 0,045 V manuell gesetzt. Cantilever: ScanAsystAir. 


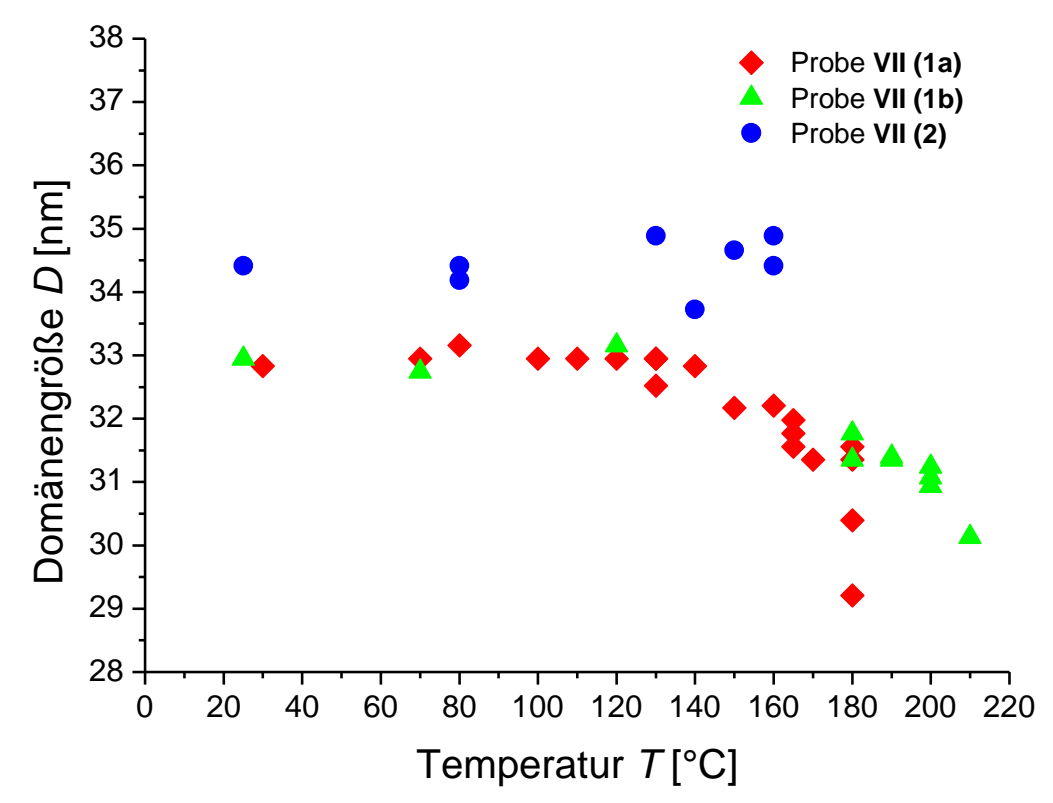

Abbildung 4-33 Entwicklung der Domänenabstände der Zylinder in Abhängigkeit von der Temperatur.

\section{Fazit}

Insgesamt erfolgte mittels AFM-Untersuchungen eine umfassende Charakterisierung der MPS für das hergestellte Blockcopolymersystem P(MMA-co-CMA)- $b$ PBA, wobei die BCP eine vielfältige Morphologie in Abhängigkeit von ihrer jeweiligen Zusammensetzung aufweisen. Um einen Einfluss der eingebauten Coumarin-Einheiten auf die MPS ausschließen zu können, wurde vergleichsweise das BCP PMMA- $b$-PBA ohne Coumarin-Anteil analysiert, wobei eine ähnliche Morphologie für beide BCP-Systeme je nach Zusammensetzung ihrer Blockkomponenten im System nachgewiesen werden konnte. Somit wirkt sich der Einbau kleiner Mengen von photo-vernetzbaren Coumarin-Anteilen in das BCP nicht auf die MPS des Systems aus. Zusätzlich wurde das Phasenverhalten des Polymers P(MMA-co-CMA)- $b$-PBA (Probe VII) bei erhöhten Temperaturen mittels in-situ-AFM-Untersuchungen analysiert, wobei das BCP-System in einem Temperaturbereich von $21^{\circ} \mathrm{C}$ bis $210^{\circ} \mathrm{C}$ in einem stabilen phasenseparierten Zustand vorliegt. Der Ordnungs-Unordnungs-Zustand (ODT) für das System wurde in diesem Temperaturbereich nicht festgestellt, da der ODT bei langkettigen Blockcopolymeren oft weit über der thermischen Stabilitätsgrenze liegt. Lediglich eine Abnahme der Zylinderabstände konnte ab einer Temperatur von ca. $180{ }^{\circ} \mathrm{C}$ beobachtet werden, wobei das vollständige Auflösen der Zylinderstruktur ausblieb. Eine weitere Möglichkeit zur Erreichung des ODTs besteht in der 
Veränderung des Polymersystems, z. B. durch Verringerung des Polymerisationsgrades $N$ des bestehenden Blockcopolymers P(MMA-co-CMA)- $b$-PBA (siehe Abschnitt 4.8) oder durch Variation der unterschiedlichen Blockkomponenten. Letzterer Fall wird in dem folgendem Abschnitt diskutiert.

\subsubsection{Untersuchung des Blockcopolymersystems P(MMA-co-CMA)- $b$-PBMA}

In diesem Abschnitt wird die MPS des Blockcopolymersystems P(MMA-co-CMA)- $b$ PBMA mit Hilfe des AFMs bestimmt. Die folgenden AFM-Untersuchungen wurden zusätzlich bei erhöhten Temperaturen durchgeführt, um einen eventuellen Übergang des BCPs von einer geordneten Phase in eine gemischte, homogene Phase beobachten zu können. Damit die Probenoberfläche nicht durch die Krafteinwirkung des Cantilevers geschädigt wird (besonders bei hohen Temperaturen), wurde der PF setpoint für die Messungen automatisch vom AFMMessprogramm eingestellt.

Abbildung 4-34 zeigt exemplarisch zwei Höhenprofile für den zweiten AufheizZyklus der Probe XI bei $21^{\circ} \mathrm{C}$ (Raumtemperatur, RT) und $110^{\circ} \mathrm{C}$. Bei dem ersten Aufheiz-Zyklus wurde die AFM-Probe von RT auf $110^{\circ} \mathrm{C}$ und bei dem zweiten Zyklus von RT auf $190^{\circ} \mathrm{C}$ aufgeheizt. Da sich die Höhenprofile bei den unterschiedlichen Temperaturen ähneln, werden im Folgenden ausgewählte Bilder mit einer guten Auflösung dargestellt.

Die phasenseparierte Probe XI in Abbildung 4-34 a) zeigt eine Zylinderstruktur, wobei bereits durch die molare Zusammensetzung des BCPs ( $F_{\mathrm{BMA}}=67 \mathrm{~mol} \%$; siehe Tabelle 4-19) diese Morphologie vermutet werden konnte. Aus der lokalen Charakterisierung der mechanischen Eigenschaften folgt, dass die höher gelegenen Objekte die P(MMA-co-CMA)-Zylinder sind, die sich liegend in einer PBMA-Matrix (dunkle Phase) anordnen. Die Zylinder erscheinen, wie bei Probe VII, in einer mäanderförmigen Struktur mit verschiedenen Kontaktpunkten und Biegungswechseln. Allerdings sind bei dem zweiten Aufheiz-Zyklus der Probe XI [Abbildung 4-34 a) und b)] kleine teilweise geschlossene P(MMA-co-CMA)-Flächen zu erkennen (weiß eingerahmt). In diesen Bereichen kann es zu einer Störung der Gleichgewichtsmorphologie kommen, da das schnelle Abkühlen der Probe nach dem ersten Aufheiz-Zyklus zu einer unvollständigen Anordnung der Blöcke führen kann. Bei der Untersuchung der Probe XI in dem Temperaturbereich der AFM- 
Heizzelle konnte kein Ordnungs-Unordnungs-Übergang festgestellt werden. Der ermittelte Durchschnittsabstand zwischen den Zylindern liegt bei $35 \mathrm{~nm}$ $\left(f=28,4 \mu \mathrm{m}^{-1}\right)$, wobei die PSD-Verteilung des Höhenprofils für Probe $\mathbf{X I}$ in Abbildung B-5 (Anhang B) dargestellt ist.
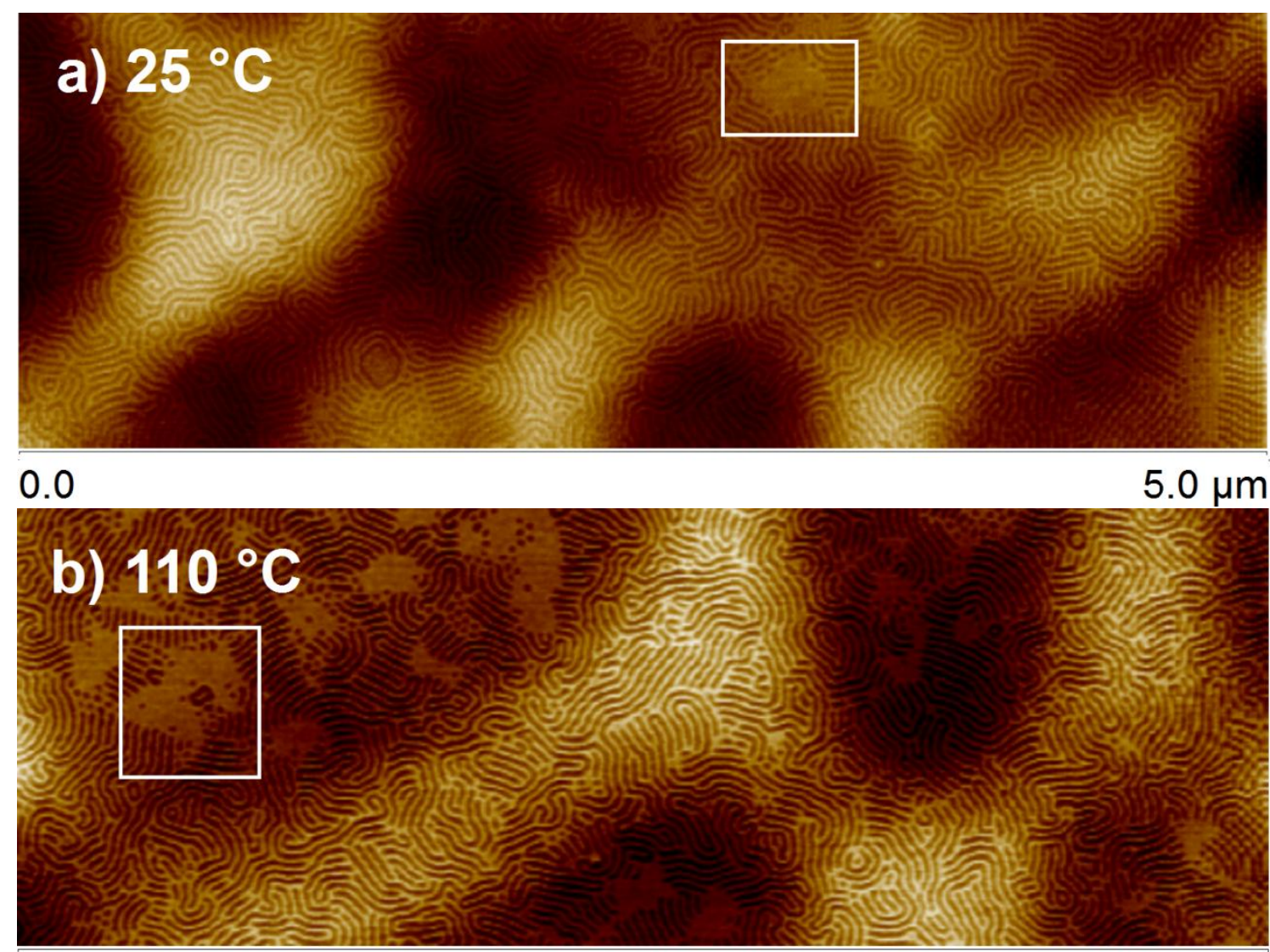

0.0

$5.0 \mu \mathrm{m}$

Abbildung 4-34 AFM-Höhenprofile von Probe XI für den zweiten Aufheiz-Zyklus: a) bei $21^{\circ} \mathrm{C}$ nach dem ersten Aufheiz-Zyklus; b) bei $110^{\circ} \mathrm{C}$. Cantilever: FESPA. PF setpoint automatisch gesetzt. Auflösung jew. 512 x 512 Pixel. Die weißen Rahmen veranschaulichen die kleinen geschlossenen P(MMA-co-CMA)-Bereiche.

Eine weitere Probe des Blockcopolymersystems P(MMA-co-CMA)- $b$-PBMA wurde in einem Temperaturbereich von $21^{\circ} \mathrm{C}$ (RT) bis $150{ }^{\circ} \mathrm{C}$ mittels AFM untersucht. Abbildung 4-35 zeigt exemplarisch das AFM-Höhenprofil der Probe XII bei $150{ }^{\circ} \mathrm{C}$. Durch das Einsetzen des thermischen Rauschens bei sehr hohen Temperaturen (siehe Abschnitt 6.6.3), konnte die Probe insgesamt nur bis $150{ }^{\circ} \mathrm{C}$ aufgeheizt werden. In Abbildung 4-35 ist für Probe XII eine Sphärenstruktur zu erkennen, wobei die regelmäßig angeordneten P(MMA-co-CMA)-Sphären in einer PBMAMatrix (dunkle Phase) eingebettet sind. Bei erhöhten Temperaturen wurde für die 
Probe kein Übergang in die homogene Phase festgestellt. Der Domänenabstand $D$ der Sphären wurde mittels PSD-Funktion (siehe Anhang B, Abbildung B-6) ermittelt und liegt bei ca. $39 \mathrm{~nm}\left(f=25,4 \mu \mathrm{m}^{-1}\right)$.

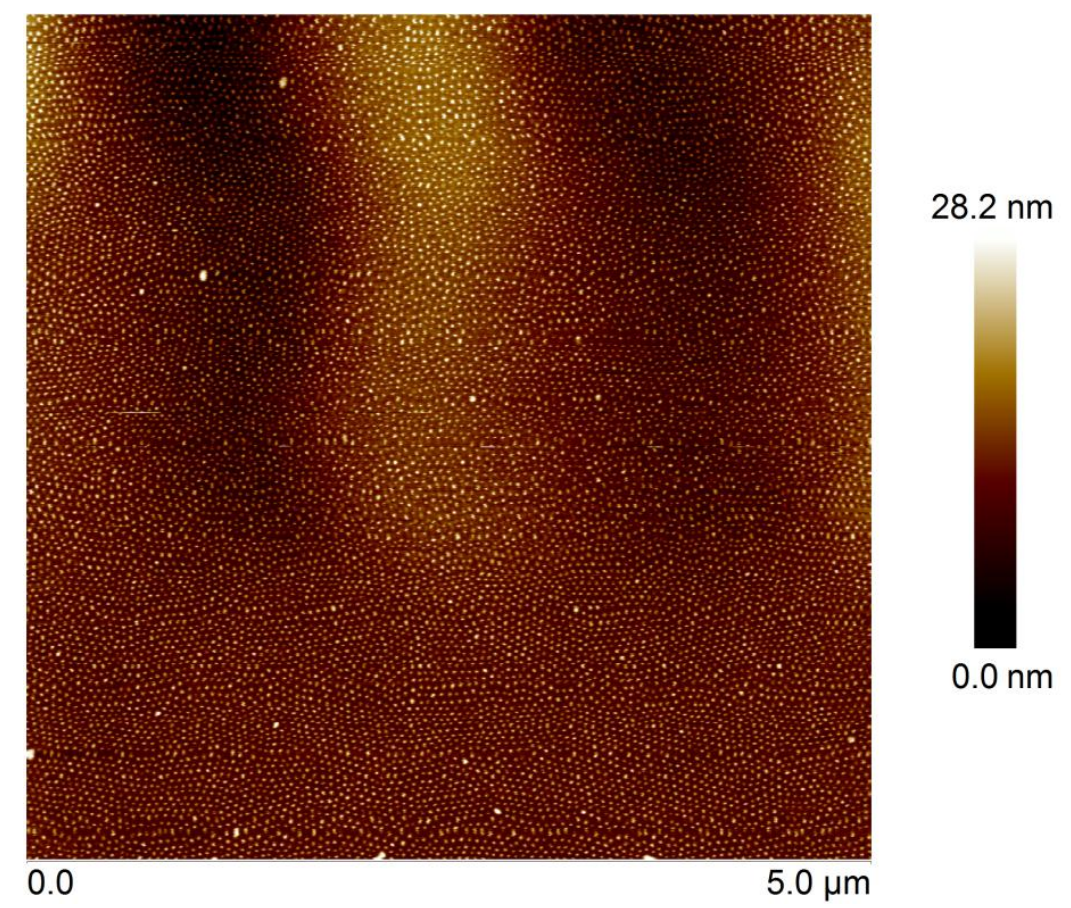

Abbildung 4-35 AFM-Höhenprofile von Probe XII bei $150^{\circ} \mathrm{C}$ für den ersten Aufheiz-Zyklus. Cantilever: FESPA. PF setpoint automatisch gesetzt. Auflösung jew. 512 x 512 Pixel.

Die Domänenabstände beider Proben (XI, XII) weichen minimal voneinander ab, wobei beide BCP denselben Ausgangsblock mit einer identischen Molmasse besitzen (siehe Tabelle 4-15). Tabelle 4-22 fasst nochmals die Zusammensetzungen der unterschiedlichen BCP, sowie die ermittelten Domänenabstände der P(MMA-co-CMA)-Phase zusammen.

Tabelle 4-22 Morphologie der synthetisierten BCP [P(MMA-co-CMA)- $b$-PBMA].

\begin{tabular}{|l|l|l|l|}
\hline Probe & $\boldsymbol{F}_{\text {BMA }}[\mathbf{m o l} \%]$ & $\boldsymbol{D}[\mathbf{n m}]$ & Morphologie \\
\hline XI & 64 & 35 & Zylinder \\
\hline XII & 91 & 39 & Sphären \\
\hline
\end{tabular}

\section{Fazit}

Dünne Polymerfilme des synthetisierten Blockcopolymersystems P(MMA-coCMA)- $b$-PBMA konnten mittels AFM analysiert werden und zeigen eine MPS mit 
einer vielfältigen Morphologie in Abhängigkeit von der Zusammensetzung des BCPs. Trotz der Ähnlichkeit der eingebauten Monomereinheiten (Methacrylate) in dem BCP und der damit verbundenen geringeren Entmischungstendenz des Materials konnte für das System kein ODT bei höheren Temperaturen ermittelt werden. Eine Möglichkeit zur Verschiebung des ODTs zu geringeren Temperaturen wäre die Synthese des BCPs P(MMA-co-CMA)- $b$-PMMA, da dieses Material im Vergleich zu den anderen Systemen die kleinste Entmischungstendenz besitzt. Eine weitere Möglichkeit zur Erreichung des ODTs besteht in der Verringerung des Polymerisationsgrads $N$ des BCPs, wobei dieser Fall für das Polymersystem P(MMA-co-CMA)-b-PBA untersucht wurde und die Diskussion in Abschnitt 4.8 erfolgt.

\subsection{Einfluss der Temperbedingungen auf die Mikro- phasenseparation}

Die in Abschnitt 4.1 und 4.2 erläuterte Synthese zeigt, dass die RAFTPolymerisation eine exakte Kontrolle der molekularen Architektur der Polymere ermöglicht. Darüber hinaus kann mit Hilfe des Temperprozesses die Struktur und Dynamik des BCP-Systems polymerphysikalisch kontrolliert werden. Zur Erreichung einer klaren MPS der einzelnen Blöcke im Polymersystem müssen diese zunächst bei einer hohen Temperatur getempert werden. Durch die Erwärmung der BCP auf eine Temperatur oberhalb ihrer jeweiligen $T_{\mathrm{g}}$ erhöht sich die Kettenbeweglichkeit des Polymers, wodurch sich die Morphologie der jeweiligen BCP im thermodynamischen Gleichgewichtszustand ausbilden kann. Somit stellen die Temperbedingungen einen wichtigen Einflussfaktor für die Ausbildung der MPS der BCP dar (siehe Abschnitt 2.5.3).

In Abschnitt 4.3 wurde das thermische Verhalten der in dieser Arbeit synthetisierten BCP näher erläutert. Die Glasübergangstemperaturen der Polymere weisen insgesamt eine breite Spanne auf, von minimal $-40^{\circ} \mathrm{C}$ bis maximal $120^{\circ} \mathrm{C}$. Um eine Kettenbeweglichkeit der Polymere und somit eine Anordnung der Strukturen garantieren zu können, wurden die BCP bei Temperaturen oberhalb der $T_{\mathrm{g}}$ der beiden Blöcke $\left(T>120^{\circ} \mathrm{C}\right)$ getempert. Um den Effekt des Temperprozesses zu veranschaulichen, wurde die Oberfläche vom BCP $\mathrm{P}\left(\mathrm{MMA}_{390}-\mathrm{Co}-\mathrm{CMA}_{29}\right.$ )- $b$-PBA526 (Probe VI) mittels AFM vor und nach dem Tempern bei $160{ }^{\circ} \mathrm{C}$ untersucht. Abbildung 4-36 zeigt die jeweiligen Höhenprofile. 

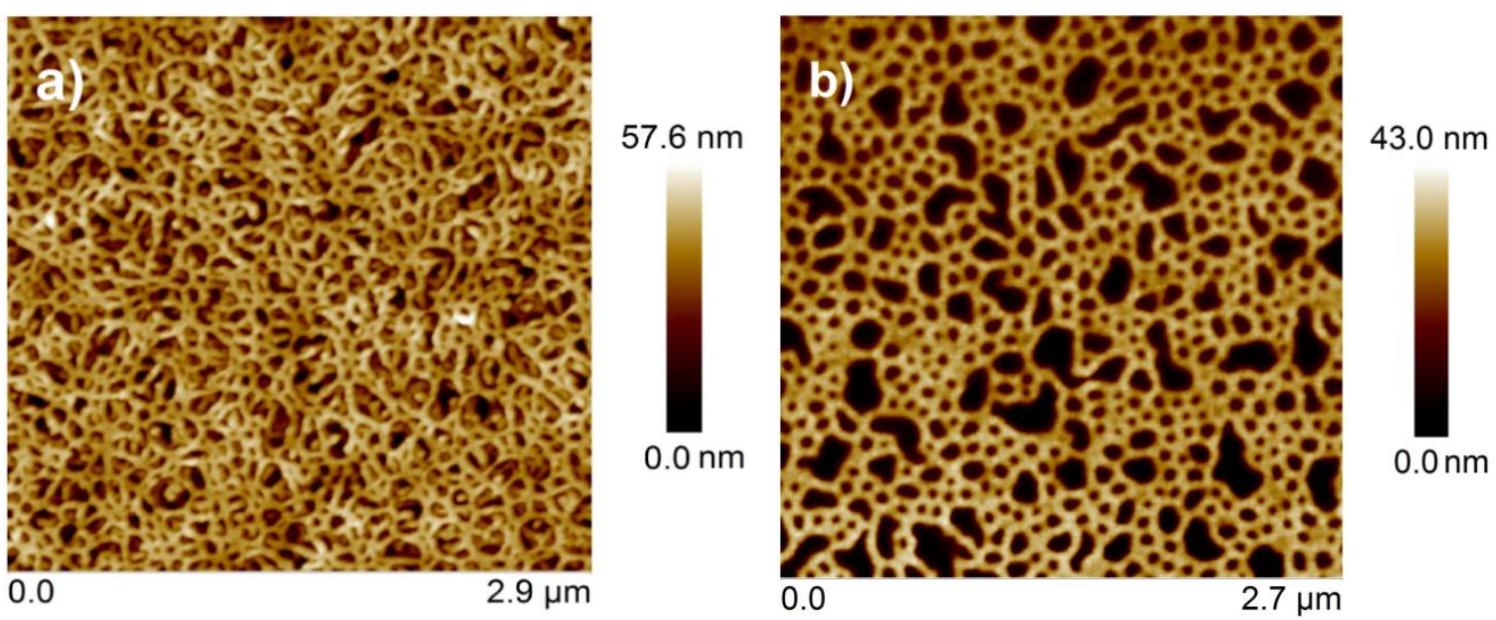

Abbildung 4-36 AFM-Höhenprofile von Probe VI: a) vor dem Tempern; b) nach dem Tempern bei $160^{\circ} \mathrm{C}$ über Nacht. Cantilever: FESPA. PF setpoint automatisch gesetzt. Auflösung jew. 512 x 512 Pixel.

In Abbildung 4-36 a) besteht die Oberfläche vor dem Tempern aus ungeordneten „Spagetti-förmigen“ P(MMA-co-CMA)-Strängen, wobei der zweite Block (PBA) nicht eindeutig erkennbar ist. Der durch das Spincoaten präparierte, mikrophasenseparierte Polymerfilm befindet sich in einem thermodynamischen Nichtgleichgewichtszustand, der bis zum Tempern stabil ist. Nach der Temperaturbehandlung (Tempern) oberhalb der $T_{\mathrm{g}}$ der beiden Blöcke ordnen sich die $\mathrm{P}(\mathrm{MMA}-\mathrm{co}$-CMA)-Domänen in einer unregelmäßigen Formation perforierter Lamellen an, die flach und parallel zum Substrat in einer darunterliegenden Domäne (PBA) eingebettet vorliegen. Während des Temperns kann das Polymersystem relaxieren und das BCP befindet sich im Gleichgewichtszustand. Die Zuweisung der unterschiedlichen Blöcke erfolgte durch Bestimmung der EModuln aus dem jeweiligen DMT-Profil (Abbildung 4-46, Abschnitt 4.6.2). Nach dem Tempern wird ein signifikanter Höhenunterschied deutlich [Abbildung 4-36 b)] und die Domänen des weichen PBA-Blocks ordnen sich in unterschiedlich großen Bereichen [dunkle Gebiete in Abbildung 4-36 b)] an. Die P(MMA-co-CMA)Phase hat sich zu einer schwammartigen Trennwand angeordnet und grenzt die dazwischen liegenden PBA-Bereiche mit einer einheitlichen Trennwandstärke voneinander ab. Die beiden AFM-Profile in Abbildung 4-36 veranschaulichen, dass der Temperprozess einen großen Einfluss auf die Morphologie hat. Das thermodynamische Gleichgewicht wurde nach einer Temperzeit von $12 \mathrm{~h}$ bei $160^{\circ} \mathrm{C}$ erreicht und somit hat weiteres Tempern keine zusätzlichen Auswirkungen auf die Morphologie. Um den Effekt des Temperns genauer zu analysieren, wurden 
die Tempertemperaturen sowie die Temperzeiten variiert. Die Ergebnisse dieser Untersuchungen sollen in den folgenden Unterkapiteln erläutert werden.

\subsubsection{Defektdefinition für Probe P(MMA95-co-CMA9)-b-PBA229 [VII]}

Der Einfluss des Tempervorgangs wurde an der mäanderförmigen Struktur des BCPs $\mathrm{P}\left(\mathrm{MMA}_{95}-\mathrm{Co}-\mathrm{CMA} 9\right)-b-\mathrm{PBA}_{229} \quad$ (VII, $\quad F_{\mathrm{BA}}=68 \mathrm{~mol} \%$ ) analysiert. Für die quantitative Beschreibung der Zylinderstruktur aus Abbildung 4-26 b) (siehe Abschnitt 4.4.1) wurden Defekte definiert. Die Anordnung der parallel zur Oberfläche liegenden Zylinder wurde als Idealstruktur angenommen und die Abweichungen von dieser Idealstruktur wurden als Defekte definiert. Dabei konnten drei Defektklassen festgestellt werden: Kurven, Kreuzungen und Sackgassen. Abbildung 4-37 stellt alle Defektklassen exemplarisch dar.

Zylinder, die sich um $180^{\circ}$ biegen, werden als Kurven definiert. Zylinder, die sich um weniger als $180^{\circ}$ biegen, sind Krümmungen und werden nicht zur Klasse der Kurven gezählt, da sie aus definierten Defekten resultieren. Die Kreuzungen sind Verbindungen von mindestens drei Zylindern. Die Endpunkte der Zylinder werden als Sackgassen definiert. Diese Sackgassen können dabei einzeln [Abbildung 4-37 c)] oder auch in einer großen Anzahl [Abbildung 4-37 d)] auftreten. Sackgassen sind dabei nicht zu verwechseln mit Kurven, da beide auf den ersten Blick eine gewisse Ähnlichkeit besitzen. Die Sackgasse kann zusätzlich einen Abgang darstellen, wobei der Zylinder nicht endet, sondern in die Probe abtaucht. Da mit dem AFM allerdings nur die Untersuchung der Oberfläche möglich ist, kann keine Aussage zu den unteren Polymerschichten getroffen werden und somit kann ein Unterschied zwischen einer Sackgasse und einem Abgang nicht getroffen werden. 

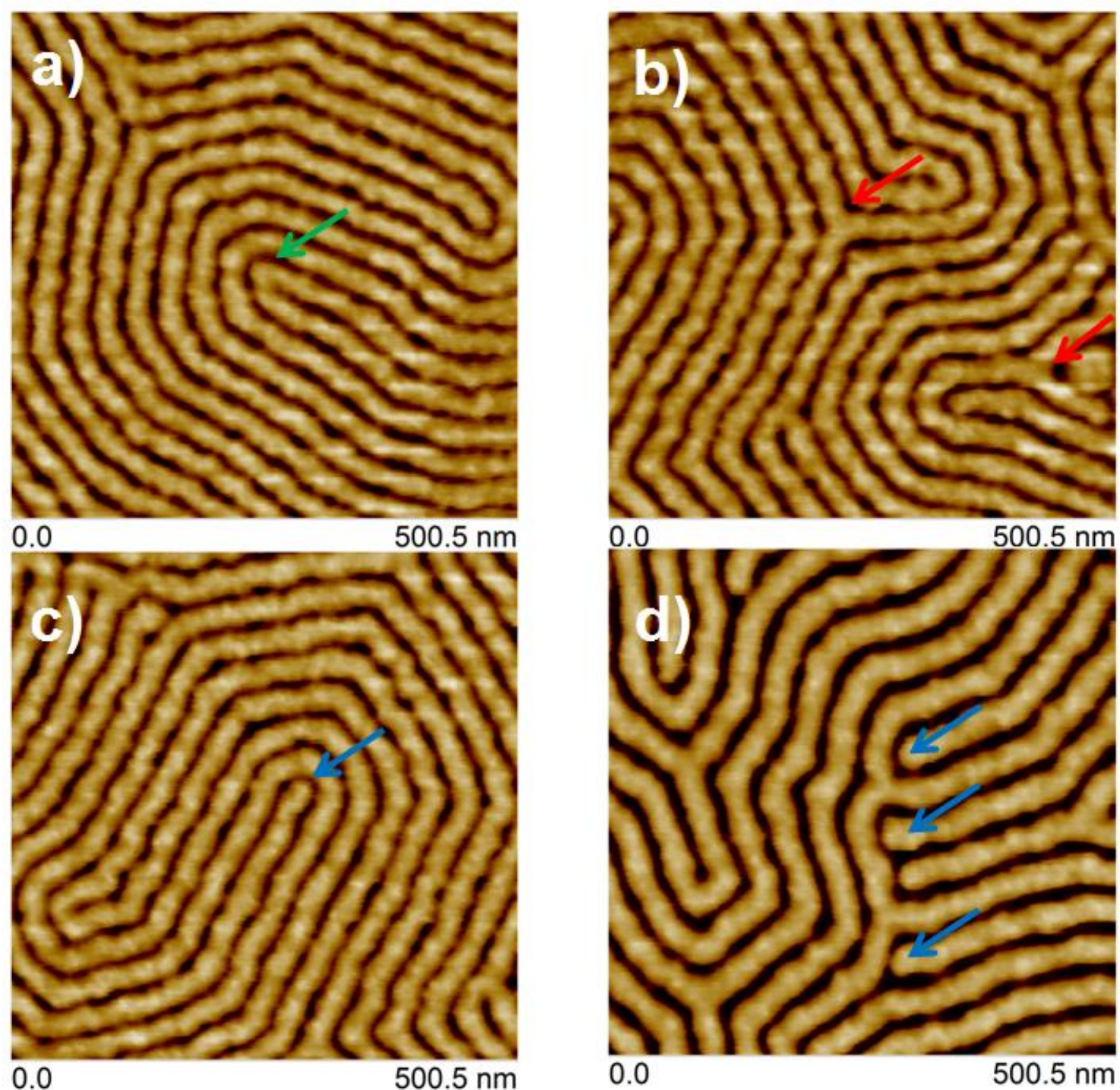

Abbildung 4-37 Definition der Defektklassen für Probe VII: a) Kurven (grüner Pfeil); b) Kreuzungen (roter Pfeil); c) und d) Sackgassen (blauer Pfeil). Cantilever: ScanAsystAir. Auflösung jew. 512 x 512 Pixel.

\subsubsection{Abhängigkeit von der Temperzeit}

Das BCP P(MMA30-co-CMA)- $b$-PBA70 (Probe VII) wurde bei $160^{\circ} \mathrm{C}$ in einem Vakuumofen getempert, wobei die Temperzeiten von $0,5 \mathrm{~h}$ bis $4 \mathrm{~h}$ variierten. Die Oberflächen der Proben wurden im Anschluss an das Tempern mittels AFM untersucht, wobei für alle Proben möglichst identische Bedingungen geschaffen wurden. So wurden für alle Messungen der PF setpoint konstant bei 0,045 V gehalten und der Cantilever ScanAsystAir verwendet. In Abbildung 4-38 sind die unterschiedlichen Höhenprofile mit den dazugehörigen Höhenverteilungskurven 
dargestellt. Die Verteilungskurven verdeutlichen, wie oft eine bestimmte Höhe in dem jeweiligen Höhenprofil zu sehen ist. Bei den normierten Verteilungen nimmt das Integral der gesamten Kurve den Wert eins an, wodurch der Anteil $\rho$ einer Höhe im Bild eine relative Größe ohne Einheit darstellt. Um die Verteilungen besser miteinander vergleichen zu können, wurde das globale Maximum der Höhe jeder Verteilung auf $0 \mathrm{~nm}$ gesetzt. Dabei ist anzumerken, dass es sich bei den AFMMessungen nur um relative Höhen handelt. Abbildung 4-38 a) zeigt das per spincoating erhaltene, nicht getemperte Ausgangsmaterial P(MMA95-co-CMA9)- $b$ $\mathrm{PBA}_{229}$ (VII). Die Probenpräparation für das AFM ist in Abschnitt 6.6.3 zu finden. Zusätzlich anzumerken ist, dass die Proben in einem Vakuumofen aufgeheizt wurden, der die Temperatur von $160{ }^{\circ} \mathrm{C}$ erst nach ca. 40 min erreichte.

Bei dem nicht getemperten Ausgangsmaterial in Abbildung 4-38 a) ist deutlich erkennbar, dass eine MPS mit einer gewissen Unregelmäßigkeit schon angedeutet wird, aber die Phasen noch nicht vollständig orientiert vorliegen. Die Verteilungskurve in Abbildung 4-38 a) zeigt ein breit verteiltes Maximum. Nach einer Temperzeit von $30 \mathrm{~min}$, wobei der in der Aufheizphase befindliche Vakuumofen in dieser Zeit eine Temperatur von ca. $125{ }^{\circ} \mathrm{C}$ angenommen hat, ist im Höhenprofil von Abbildung 4-38 b) eine relativ homogene Oberfläche zu erkennen, sowie eine zunehmende Entmischung der Phasen. Dies wird durch die Bildung einer Schulter in der Verteilungskurve bestätigt. Ein signifikanter Unterschied ist schon nach einer Temperzeit von $1 \mathrm{~h}$ zu erkennen. Das Höhenprofil in Abbildung 4-38 c) zeigt die zunehmende Orientierung der Zylinder und in der Höhenverteilungskurve sind nun zwei Maxima zu erkennen. Dabei entspricht das erste kleinere Maximum der Höhe der Täler (dunkler Bereich im Profil) und das zweite Maximum bei $0 \mathrm{~nm}$ der Höhe der Zylinder (heller Bereich). Bei den Proben in Abbildung 4-38 c) bis e) ist mit zunehmender Temperzeit eine Abnahme der Defektzahl zu erkennen und mit zunehmender Orientierung der Domänen vergrößern sich die Bereiche parallel verlaufender Zylinder. Die Defektentwicklung in Abhängigkeit von der Temperzeit und -temperatur wird in Abschnitt 4.5.4 näher erläutert. Weiterhin nähern sich die Maxima in den Höhenverteilungen mit zunehmender Temperzeit an, d. h. die Höhendifferenz zwischen den Zylindern und den Tälern nimmt leicht $\mathrm{ab}$. Ab einer Temperzeit von $2 \mathrm{~h}$ [Abbildung 4-38 f) und g)] sind sowohl im Höhenprofil als auch in der Verteilungskurve keine signifikanten Veränderungen mehr zu erkennen. Zusätzlich wurde bei allen Proben in Abbildung 4-38 der Domänenabstand der Zylinder mittels PSD-Funktion bestimmt, wobei insgesamt für alle Proben ein Zylinderabstand von ca. $30 \mathrm{~nm}$ festgestellt wurde. Da der Wert für alle 
Proben konstant bleibt, konnte keine Abhängigkeit des Domänenabstands von der Temperzeit festgestellt werden.

Zusätzlich zu den Höhenprofilen werden in Abbildung 4-39 die Profile des DMTModuls mit den zugehörigen Verteilungskurven dargestellt. Für den DMT-Modul wurde keine Kalibration vorgenommen, wodurch der Modul mit der Einheit Volt angegeben wird und als relativer Wert zu betrachten ist.

Die Profile des DMT-Moduls zeigen ähnliche Resultate wie die Höhenprofile in Abbildung 4-38. Das breite Maximum in der Verteilungskurve des DMT-Moduls in Abbildung 4-39 a) bestätigt eine homogene Oberfläche. So ist auch in Abbildung 4-39 die zunehmende Orientierung der Zylinder nach $1 \mathrm{~h}$ erkennbar. Mit steigender Temperzeit bildet sich ein zweites Maximum in der Verteilungskurve des DMT-Moduls, wobei das Maximum mit dem niedrigeren DMT-Modul-Wert die Täler repräsentiert (PBA-Domäne) und das Maximum mit dem höheren Wert den P(MMA-co-CMA)-Zylindern entspricht (helle Bereiche). Die Form der Verteilungskurven der Proben in Abbildung 4-39 c) bis g) bleibt bestehen, nur die relativen Werte des DMT-Moduls schwanken ohne sichtbare Tendenz.

Die nicht getemperte Probe in Abbildung 4-39a) wurde durch das Spincoaten einer verdünnten Polymerlösung auf ein Substrat hergestellt. Das Höhenprofil sowie der DMT-Modul spiegeln eine homogene Oberfläche wider, wodurch eine im Vergleich zu den getemperten Proben unregelmäßige Orientierung der Phasen klar zu erkennen ist. Die Kettenbeweglichkeit ist erst ab einer Temperatur oberhalb der $T_{\mathrm{g}}$ beider Blöcke gegeben und für die Ausbildung des geordneten Zustands benötigt das Polymersystem ausreichend Energie. Dies scheint bei einer Temperatur von $160{ }^{\circ} \mathrm{C}$ nach $1 \mathrm{~h}$ Temperzeit gegeben zu sein. Mit zunehmender Temperzeit vervollständigt sich die Ausbildung der Phasenseparation und die Defekte der Morphologie können ausheilen. Dieser Vorgang benötigt mehr Zeit als die eigentliche Strukturbildung, da sich für die Ausheilung wesentlich mehr Ketten auf einmal bewegen müssen und die Triebkräfte geringer sind. Als nächstes soll der Einfluss der Temperaturvariation beim Tempern erläutert werden. 

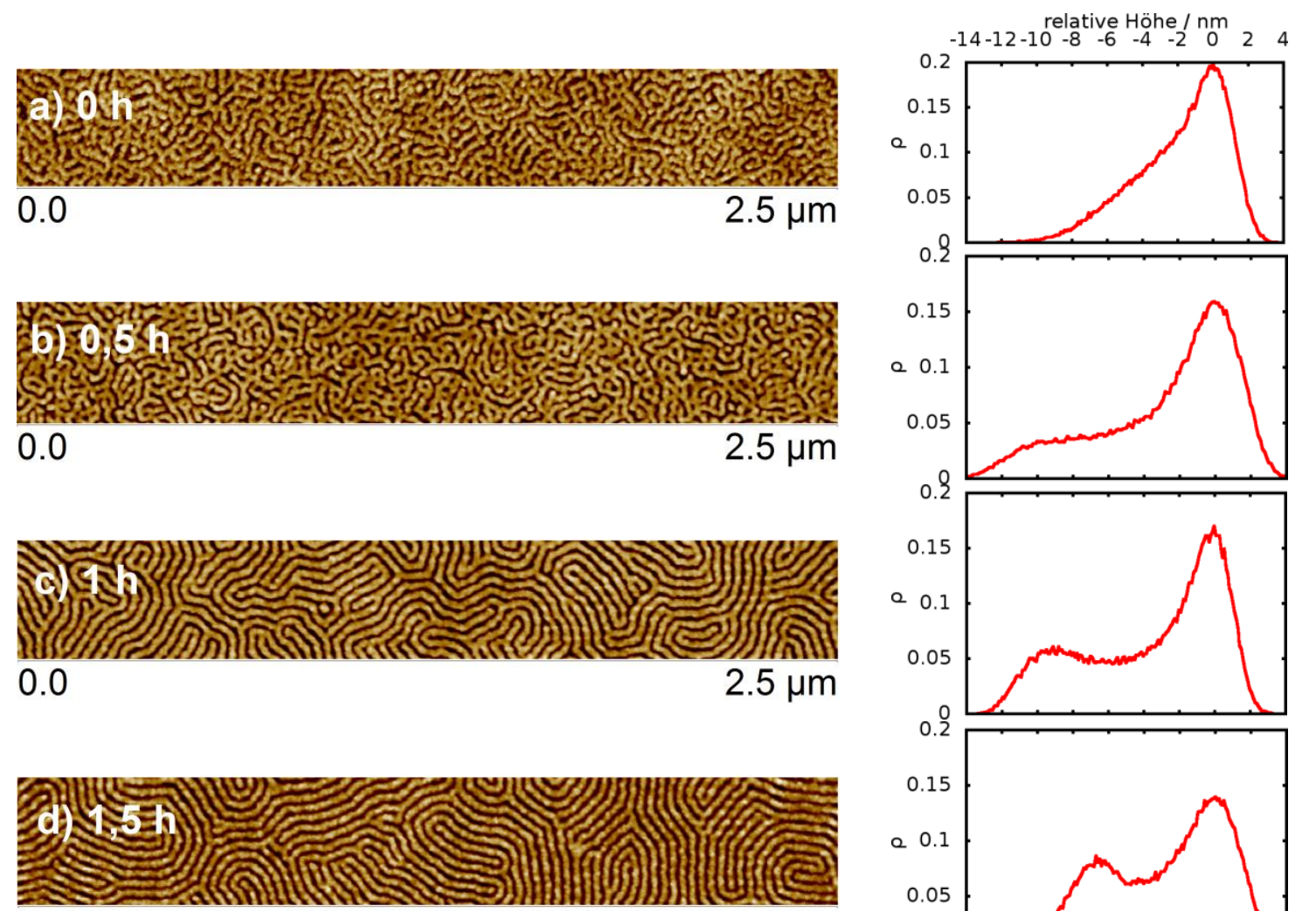

0.0

$2.5 \mu \mathrm{m}$
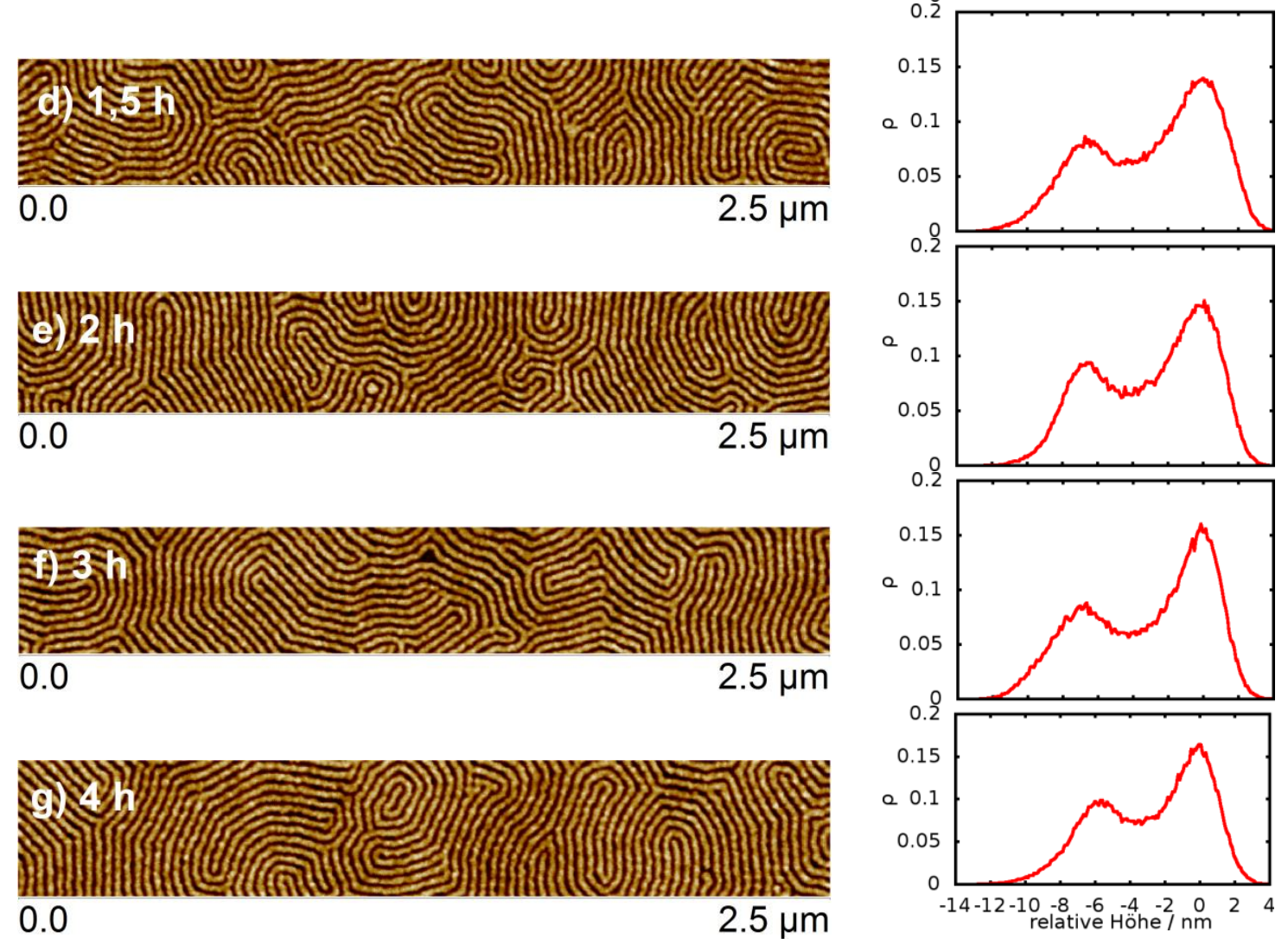

Abbildung 4-38 AFM-Höhenprofile und Höhenverteilungskurven für $\mathrm{P}\left(\mathrm{MMA}_{95}-\mathrm{co}\right.$-CMA $)-b-\mathrm{PBA}_{229}$ (VII) nach verschiedenen Temperzeiten bei $160^{\circ} \mathrm{C}$. Cantilever: ScanAsystAir. PF setpoint manuell festgelegt. Auflösung jew. 512 x 512 Pixel. 

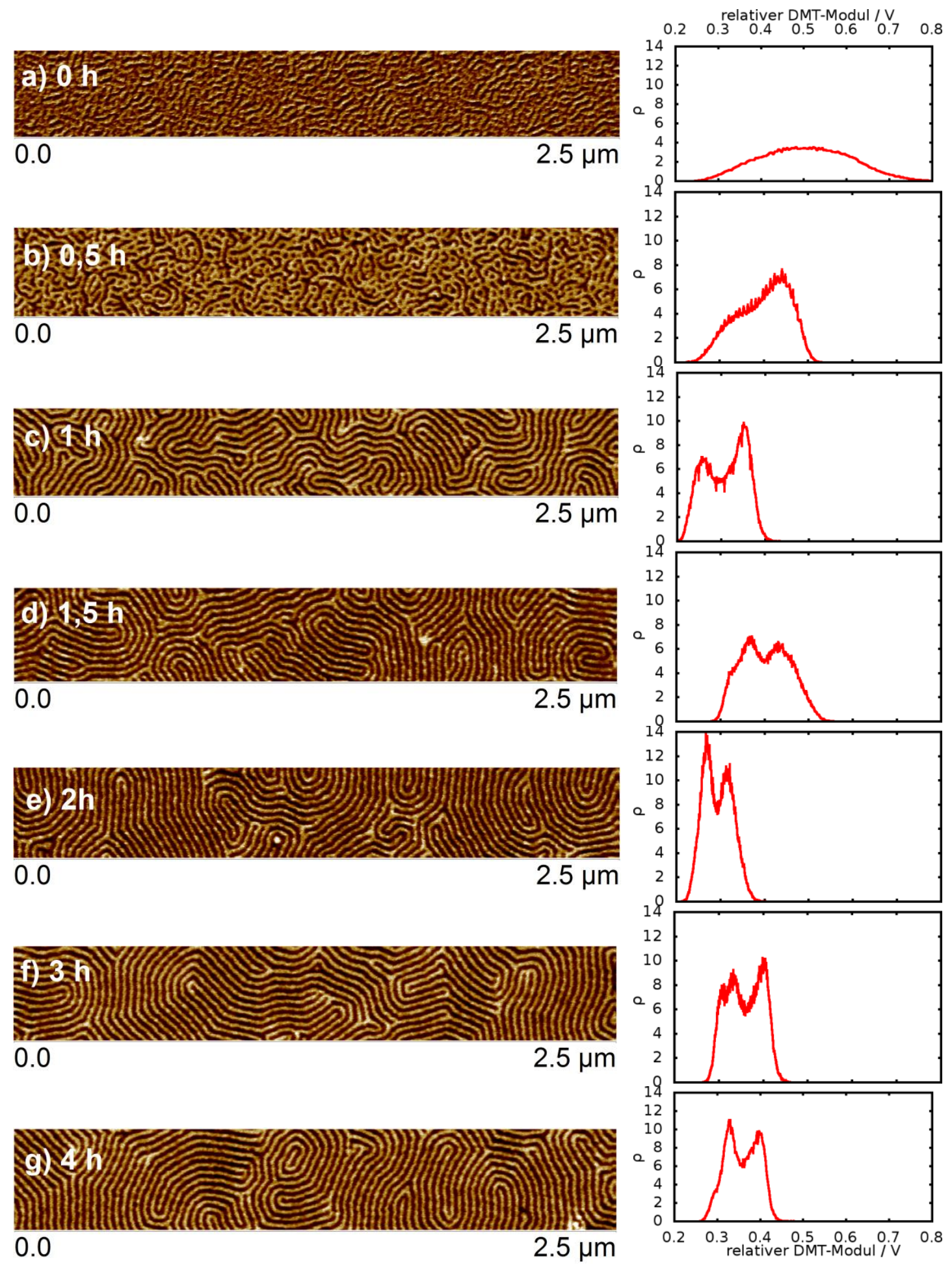

0.0

$2.5 \mu \mathrm{m}$

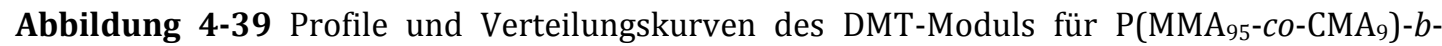
$\mathrm{PBA}_{229}$ (VII) nach verschiedenen Temperzeiten bei $160^{\circ} \mathrm{C}$. Cantilever: ScanAsystAir. PF setpoint manuell festgelegt. Auflösung jew. 512 x 512 Pixel. 


\subsubsection{Abhängigkeit von der Tempertemperatur}

Das BCP P(MMA $30-c o-\mathrm{CMA})-b-\mathrm{PBA}_{70}$ (VII) wurde bei drei unterschiedlichen Temperaturen $\left(120^{\circ} \mathrm{C}, 160^{\circ} \mathrm{C}, 180^{\circ} \mathrm{C}\right)$ in einem Vakuumofen getempert. Die Proben wurden für eine lange Zeit (15 bis $18 \mathrm{~h}$ ) getempert, um einen Einfluss der Temperzeit ausschließen zu können. Die Oberflächen der Proben wurden im Anschluss an das Tempern mittels AFM untersucht und die Höhenprofile sind in Abbildung 4-40 dargestellt.
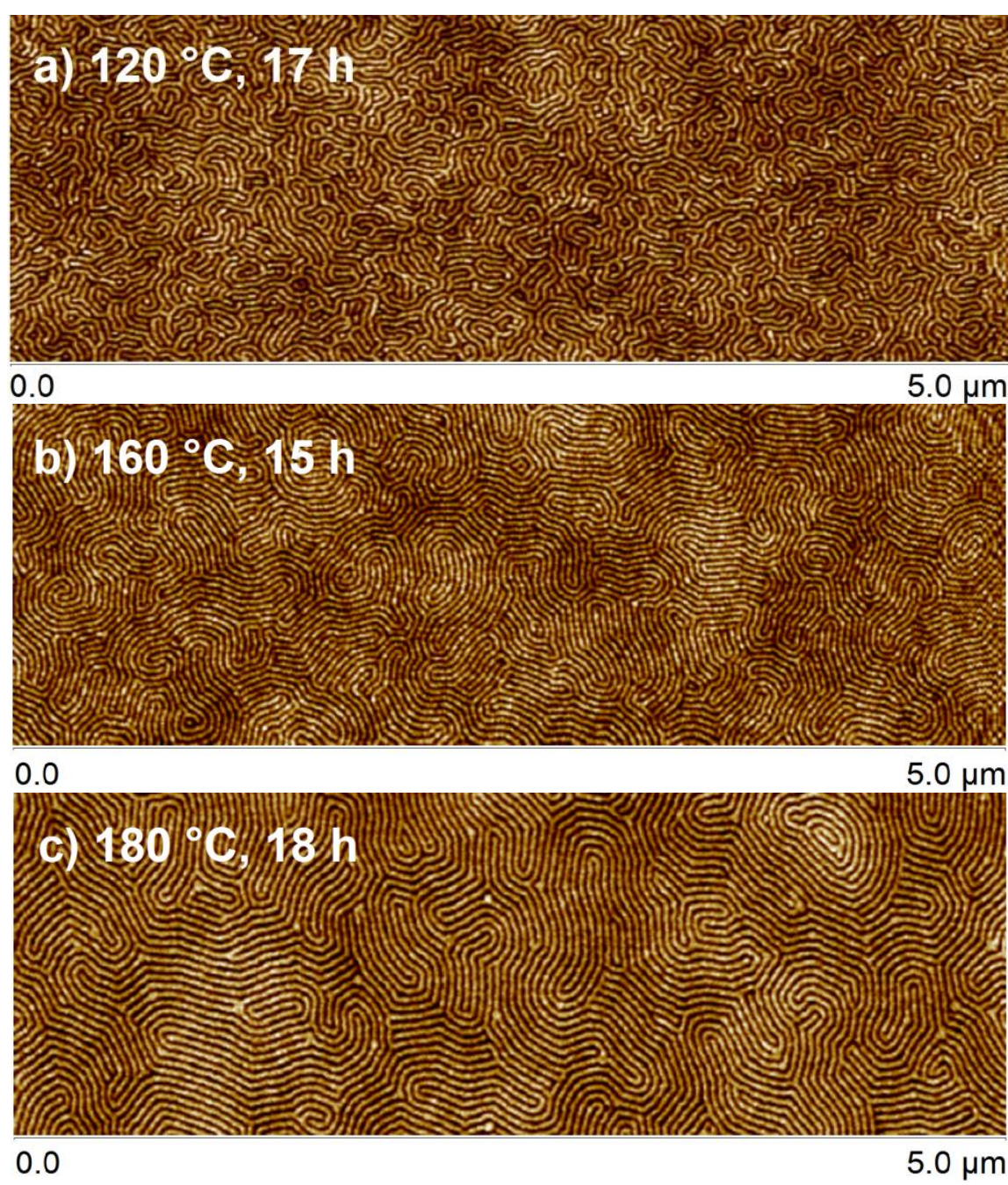

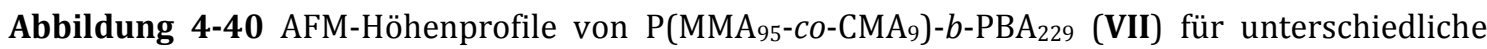
Temperaturen beim Temperprozess. Verwendete Cantilever: für a) und c) RFESP; b) ScanAsystAir. PF setpoint manuell festgelegt. Auflösung jew. 512 x 512 Pixel.

Abbildung 4-40 veranschaulicht für alle drei Proben eine Zylinderstruktur, die in unterschiedlich ausgeprägter Form vorliegt. Die Probe in Abbildung 4-40 a) wurde bei einer Temperatur von $120^{\circ} \mathrm{C}$ getempert, also im Bereich der $T_{\mathrm{g}}$ des P(MMA-co- 
CMA)-Blocks. Das Höhenprofil [Abbildung 4-40 a)] veranschaulicht eine unregelmäßige Orientierung der P(MMA-co-CMA)-Domäne und die Ausbildung von parallel verlaufenen Zylindern konnte auch nach einer Temperzeit von $17 \mathrm{~h}$ nicht erreicht werden. Folglich reicht die Energie bei dieser Temperatur noch nicht aus, um eine vollständig ausgeprägte Orientierung der MPS zu erreichen. Im Gegensatz dazu verdeutlichen die Proben in Abbildung 4-40 b) und c), dass mit zunehmender Temperatur die Bereiche parallel verlaufender Zylinder immer größer werden und weniger Defekte zu erkennen sind. Weiterhin wurden die Zylinderabstände der Proben in Abbildung 4-40 mittels PSD-Funktion bestimmt, wobei der Domänenabstand für alle Proben zwischen 32 und $33 \mathrm{~nm}$ liegt und somit nicht von der Tempertemperatur abhängt. Die Entwicklung der Defekte unter Berücksichtigung der Temperzeiten und Temperaturen soll im folgenden Unterkapitel erläutert werden.

\subsubsection{Defektentwicklung in Abhängigkeit von der Temperzeit und Temperatur}

Bei der Probe P(MMA95-Co-CMA9)- $b$ - $\mathrm{PBA}_{229}$ (VII) wurden in Abschnitt 4.5.1 unterschiedliche Defekte definiert. Zur quantitativen Betrachtung der Defekte wurden von den jeweiligen Proben in Abbildung 4-38 c) bis g) (Abschnitt 4.5.2) zusätzliche AFM-Höhenprofile mit den Maßen $3 \mu \mathrm{m}$ x $3 \mu \mathrm{m}$ aufgenommen, wobei die Proben in Abbildung 4-38 a) und b) in der Auswertung nicht berücksichtigt wurden, da die Phasen noch nicht vollständig orientiert vorliegen. Die Defekte (Kurven, Sackgassen, Kreuzungen) wurden in den Höhenprofilen der Proben c) bis g) einzeln ausgezählt. Die Ergebnisse der Auszählung sind in Abbildung 4-41 grafisch dargestellt, wobei die entsprechenden ausgezählten Höhenprofile im Anhang B in Abbildung B-7 bis Abbildung B-11 zu finden sind.

In Abbildung 4-41 ist die Entwicklung der Defektdichte in Abhängigkeit von der Temperzeit dargestellt. Dabei sind die Defektklassen farblich unterschiedlich gekennzeichnet. Die Sackgassen werden durch blaue Dreiecke dargestellt, die Kreuzungen durch rote Dreiecke und die Kurven durch grüne Dreiecke. Da die Anzahl der Kurven pro $\mu \mathrm{m}^{2}$ wesentlich geringer ist als bei den anderen Defektklassen, wurden diese mit einer anderen Skalierung aufgetragen (rechte y-Achse in Abbildung 4-41). Zusätzlich wurde in Abbildung 4-41 die Summe aller Defektklassen gegen die Temperzeit aufgetragen (schwarze Sterne). 
Insgesamt zeigt sich, dass die Defektdichte für alle Defektklassen mit zunehmender Temperzeit abnimmt. Dies bestätigt das Ergebnis der Höhenprofile in Abbildung 4-38. Die geringe Gesamtanzahl der Kurven kommt wahrscheinlich zustande, da die Bildung der Kurven energetisch sehr ungünstig ist. Bei der Kurvenbildung werden die Polymerstränge stark verzerrt, da durch die Krümmung die Innenseite des Zylinders gestaucht und die Außenseite gedehnt wird. Die anderen Defektklassen (Kreuzungen, Sackgassen) scheinen insgesamt stabiler zu sein, da die Polymerstränge bei dieser Strukturausbildung eine geringere Verzerrung aufweisen. Um eine bessere Abschätzung der Defektdichteentwicklung mit der Temperzeit treffen zu können, wurde mit dem BCP P(MMA95-co-CMA9)-b-PBA 229 (VII) ein Langzeitexperiment durchgeführt. Dementsprechend wurden mehrere Proben des BCPs in einem Zeitintervall von 2 bis $34 \mathrm{~h}$ bei $160^{\circ} \mathrm{C}$ getempert. Abbildung 4-42 zeigt den grafischen Verlauf für diese Proben.

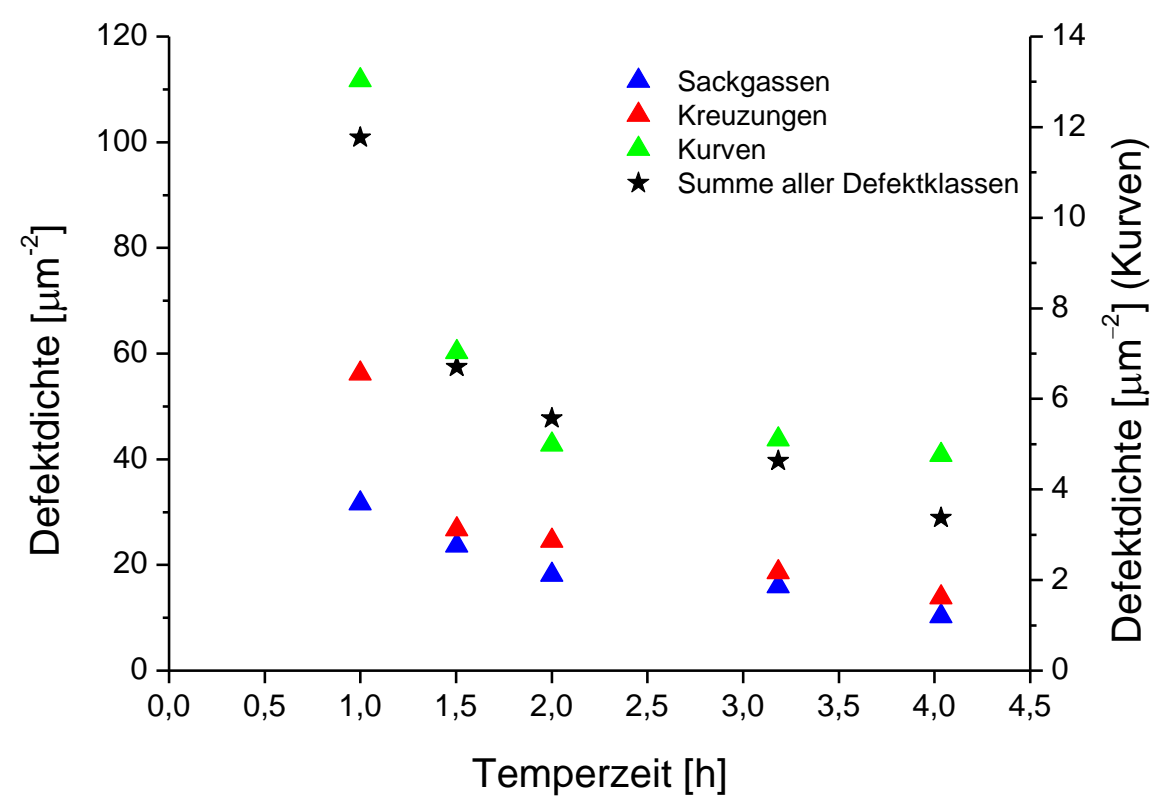

Abbildung 4-41 Entwicklung der Defektdichte mit der Temperzeit für P(MMA95-co-CMA9)- $b$ $\mathrm{PBA}_{229}$ (VII). Die wurde Probe bei $160{ }^{\circ} \mathrm{C}$ getempert. Cantilever: ScanAsystAir. 


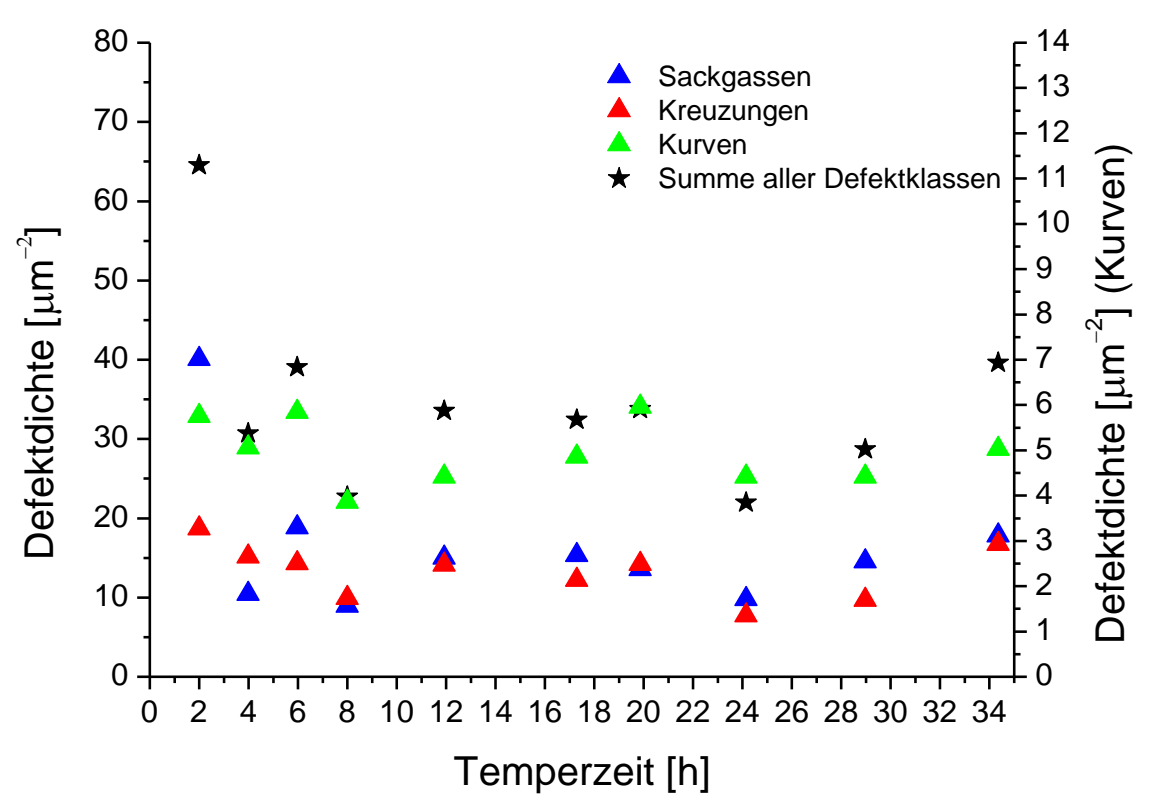

Abbildung 4-42 Entwicklung der Defektdichte in Abhängigkeit von der Temperzeit für P(MMA95co-CMA9)- $b$ - $\mathrm{PBA}_{229}$ (VII). Die Probe wurde bei $160{ }^{\circ} \mathrm{C}$ getempert. Cantilever: ScanAsystAir.

Eine Abnahme der Defektdichten ist bis zu einer Temperzeit von ca. $12 \mathrm{~h} \mathrm{zu}$ beobachten, wobei das Polymersystem im Anschluss seine Gleichgewichtsmorphologie erreicht hat. Für die Bestimmung der mittleren Defektdichte im Gleichgewicht wurde der Mittelwert der Defektdichten mit Standardabweichung für die Proben von 12 h bis 34 h (Abbildung 4-42) berechnet. Tabelle 4-23 zeigt die ermittelten Ergebnisse, wobei festzustellen ist, dass die mittlere Defektdichte der Sackgassen und Kreuzungen jeweils doppelt bis dreimal so groß ist wie die der Kurven. Ein komplettes Ausheilen des Materials konnte auch nach einer Temperzeit von ca. $34 \mathrm{~h}$ nicht festgestellt werden. Dieser Effekt kann auf die Dispersität des BCPs und Verunreinigungen der Probe zurückgeführt werden.

Tabelle 4-23 Mittlere Defektdichte von P(MMA95-co-CMA9)- $b-\mathrm{PBA}_{229}$ (VII). Die Proben wurden bei $160{ }^{\circ} \mathrm{C}$ von $12 \mathrm{~h}$ bis $34 \mathrm{~h}$ in einem Vakuumofen getempert.

\begin{tabular}{|l|l|}
\hline Defektklasse & Mittlere Defektdichte $\left[\boldsymbol{\mu m}^{-2}\right]$ \\
\hline Kurven & $5 \pm 1$ \\
\hline Sackgassen & $14 \pm 3$ \\
\hline Kreuzungen & $12 \pm 3$ \\
\hline Summe aller Defekte & $32 \pm 6$ \\
\hline
\end{tabular}


Zum Schluss soll die Defektdichte in Abhängigkeit von der Tempertemperatur betrachtet werden. In Abbildung 4-40 (Abschnitt 4.5.3) sind drei Proben des Blockcopolymers VII dargestellt, die bei unterschiedlichen Temperaturen getempert wurden. Für diese Proben wurden wiederum Höhenprofile mit den Maßen $3 \mu \mathrm{m} \times 3 \mu \mathrm{m}$ aufgenommen, in denen die Defekte für eine quantitative Bestimmung einzeln ausgezählt wurden. Die Ergebnisse sind in Abbildung 4-43 grafisch dargestellt und die entsprechenden Höhenprofile sind im Anhang B (Abbildung B-12 bis Abbildung B-14) zu finden.

Insgesamt ist eine starke Abnahme der Defektdichte aller Defektklassen mit zunehmender Tempertemperatur erkennbar. In Abschnitt 4.5.3 konnte bereits festgestellt werden, dass bei einer Temperatur von $120^{\circ} \mathrm{C}$ die zugeführte Energie für eine vollständig ausgeprägte Orientierung der MPS im System nicht ausreicht. Die hohe Defektdichte (Abbildung 4-43) bestätigt eine unregelmäßige Anordnung der Zylinder-Domänen in dem Material. Mit zunehmender Temperatur werden die Bereiche parallel verlaufender Zylinder größer und somit kommt es zu einer Abnahme der Defekte. Die Abnahme der Defektdichte mit zunehmender Temperatur ist auf die Kettenbeweglichkeit der Polymere zurückzuführen, wobei sich das System bei einer Temperatur von $120^{\circ} \mathrm{C}$ knapp oberhalb der $T_{\mathrm{g}}$ des P(MMA-co-CMA)-Blocks befindet. Durch die Temperaturerhöhung auf $160^{\circ} \mathrm{C}$ wird dem Polymersystem mehr Energie zugeführt, wodurch die Abnahme der Defektdichte begünstigt wird. Für die Probe bei $160^{\circ} \mathrm{C}$ wurden nach einer Temperzeit von $12 \mathrm{~h}$ keine signifikanten Änderungen der Defektdichte mehr festgestellt (siehe Abbildung 4-42) und das Polymersystem befindet sich in einem Gleichgewichtszustand. Gleiches gilt für die Probe bei $180^{\circ} \mathrm{C}$, wobei durch die höhere Temperatur dem System mehr Energie zugeführt wird und im Vergleich zu der Probe bei $160{ }^{\circ} \mathrm{C}$ eine geringe Abnahme der Defektdichte festgestellt wurde. Dieser Effekt kann auf die Dispersität des BCPs und Verunreinigungen der Probe zurückgeführt werden.

Die Abnahme der Defektdichte mit zunehmender Temperatur lässt darauf schließen, dass bei weiterer Temperaturzunahme die Möglichkeit für ein komplettes Ausheilen der Defekte im System besteht. Dieser Effekt wurde in der vorliegenden Arbeit allerdings nicht weiter untersucht, da mit zunehmenden Tempertemperaturen auch die Gefahr der Schädigung des Polymers besteht. Außerdem wird durch mögliche Verunreinigungen der Probe und die Dispersität des Blockcopolymers die Ausbildung einer defektfreien MPS ausgeschlossen. 


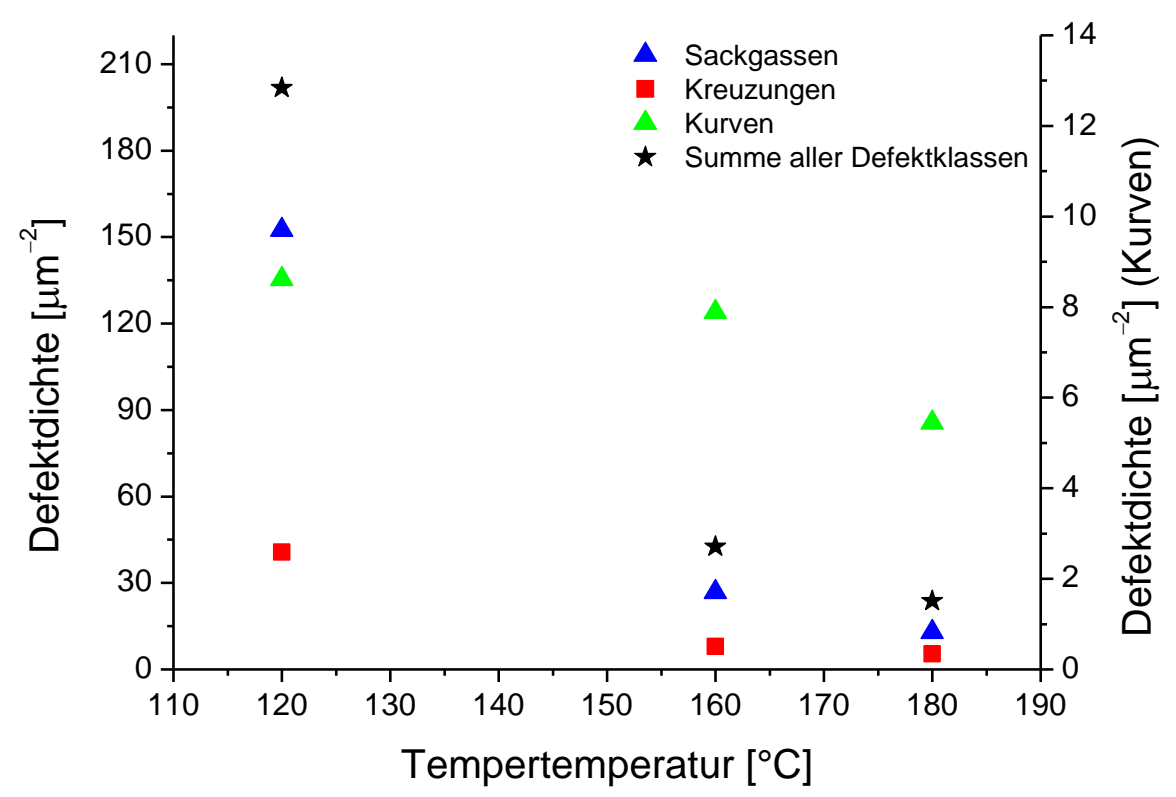

Abbildung 4-43 Entwicklung der Defektdichte in Abhängigkeit von der Tempertemperatur für $\mathrm{P}\left(\mathrm{MMA}_{95}-\mathrm{Co}-\mathrm{CMA}_{9}\right)-b-\mathrm{PBA}_{22}$ (VII). Cantilever: ScanAsystAir.

\subsubsection{Fazit}

Zusammenfassend kann festgestellt werden, dass der Tempervorgang einen außerordentlichen Einfluss auf die Ausbildung der Mikrophasenseparation hat. Sowohl die Temperzeit als auch die Tempertemperatur müssen richtig gewählt werden, um dem Polymersystem die Möglichkeit zur vollständigen Phasenseparation zu geben. Für die Ausbildung des geordneten Zustands benötigt das System ausreichend Energie und somit höhere Temperaturen, da die Beweglichkeit aller Ketten in dem Polymersystem erst ab einer Temperatur oberhalb der $T_{\mathrm{g}}$ beider Blockkomponenten erreicht wird. Mit zunehmender Temperzeit und -temperatur vervollständigt sich die Ausbildung der Phasenseparation im System und die in Probe VII festgestellten Defekte der Morphologie können ausheilen. Dieser Vorgang benötigt mehr Zeit als die eigentliche Strukturbildung, da sich für die Ausheilung der Defekte wesentlich mehr Ketten auf einmal bewegen müssen und die Triebkräfte geringer sind. Trotz alledem konnte für das BCP VII für die oben beschriebenen Temperbedingungen keine defektfreie MPS festgestellt werden. Dieser Effekt wird auf die Dispersität des Blockcopolymers und mögliche Verunreinigungen der Probe zurückgeführt. Eine Abhängigkeit des Zylinderabstandes von der Temperzeit bzw. -temperatur wurde nicht festgestellt. Insgesamt wurden für die in der vorliegenden Arbeit 
verwendeten BCP eine ausreichende Tempertemperatur von $160{ }^{\circ} \mathrm{C}$ und eine Temperzeit von mindestens $12 \mathrm{~h}$ festgestellt.

\subsection{Bestimmung mechanischer Eigenschaften}

Für die Bestimmung der mechanischen Eigenschaften der BCP auf nanoskopischer Skala wurde der AFM-Modus PeakForce-QNM ${ }^{\mathrm{TM}}$ benutzt. Bei dieser Messmethode können nicht nur topologische Informationen der Probe bestimmt werden, sondern auch mechanische Eigenschaften wie Elastizitätsmodul, Adhäsion, Deformation und Energiedissipation. Die genaue Erklärung dieses speziellen Modus ist dem Abschnitt 2.7.3 zu entnehmen. In den folgenden Unterkapiteln sollen die mechanischen Eigenschaften der Proben V, VI und VII des Blockcopolymers P(MMA-co-CMA)- $b$-PBA (siehe Tabelle 4-15, Abschnitt 4.2.3) analysiert werden, um eine qualitative Zuordnung der phasenseparierten Domänen zu den jeweiligen Blöcken treffen zu können.

\subsubsection{Verwendung von PeakForce-QNM ${ }^{\mathrm{TM}}$ bei $\mathrm{P}\left(\mathrm{MMA}_{390}-\mathrm{Co}_{\mathrm{C}}-\mathrm{CMA}_{29}\right)-$ $b$-PBA 426}

Die MPS von Probe V wurde in Abschnitt 4.4.1 ausführlich diskutiert. Durch die lokale Charakterisierung der DMT-Modul-Profile sowie Adhäsionsprofile kann eine eindeutige Zuordnung der jeweiligen Phasen getroffen werden. Abbildung 4-44 zeigt die unterschiedlichen AFM-Profile der Probe V. Das AFM-Höhenprofil von Probe $\mathbf{V}$ in Abbildung 4-44 a) zeigt, dass die höher gelegenen Domänen perforierte Lamellen bilden, die wiederum in einem darunterliegenden Bereich (dunkle Phase) eingebettet sind. Im Gegensatz zum Höhenprofil veranschaulicht das Adhäsionsprofil in Abbildung 4-44 c), dass die tiefer gelegenen Gebiete eine hohe Klebrigkeit besitzen, wohingegen die perforierten Lamellen eine geringere Adhäsion aufweisen. Bei der AFM-Messung $\left(\mathrm{T}=21^{\circ} \mathrm{C}\right)$ besitzt der PBA-Block durch seine niedrige $T_{\mathrm{g}}$ eine wesentlich höhere Klebrigkeit als der P(MMA-coCMA)-Block, wodurch eine erste Zuordnung der unterschiedlichen Bereiche getroffen werden kann. Das DMT-Modul-Profil in Abbildung 4-44 b) veranschaulicht die tiefer gelegene Phase als weichen Bereich, der von einem härteren Gebiet (perforierte Lamellen) umgeben ist. Zusätzlich zu den signifikanten Aussagen wurden die DMT-Moduln für die jeweiligen Blöcke aus dem DMT-Modul-Profil bestimmt, wobei die ausführliche Beschreibung dem Abschnitt 2.7.3 zu entnehmen ist. Die Verteilungen der DMT-Modulwerte der Oberfläche entsprechen in guter 
Näherung einer Gauß-Verteilung und wurden deshalb mit einer entsprechenden Funktion angepasst, um den Fehler anhand der Standardabweichung $\sigma \mathrm{zu}$ bestimmen. Abbildung 4-45 zeigt die Rohdaten der DMT-Modulwerte der Oberfläche und die jeweils angepasste Gaußkurve für Probe V.
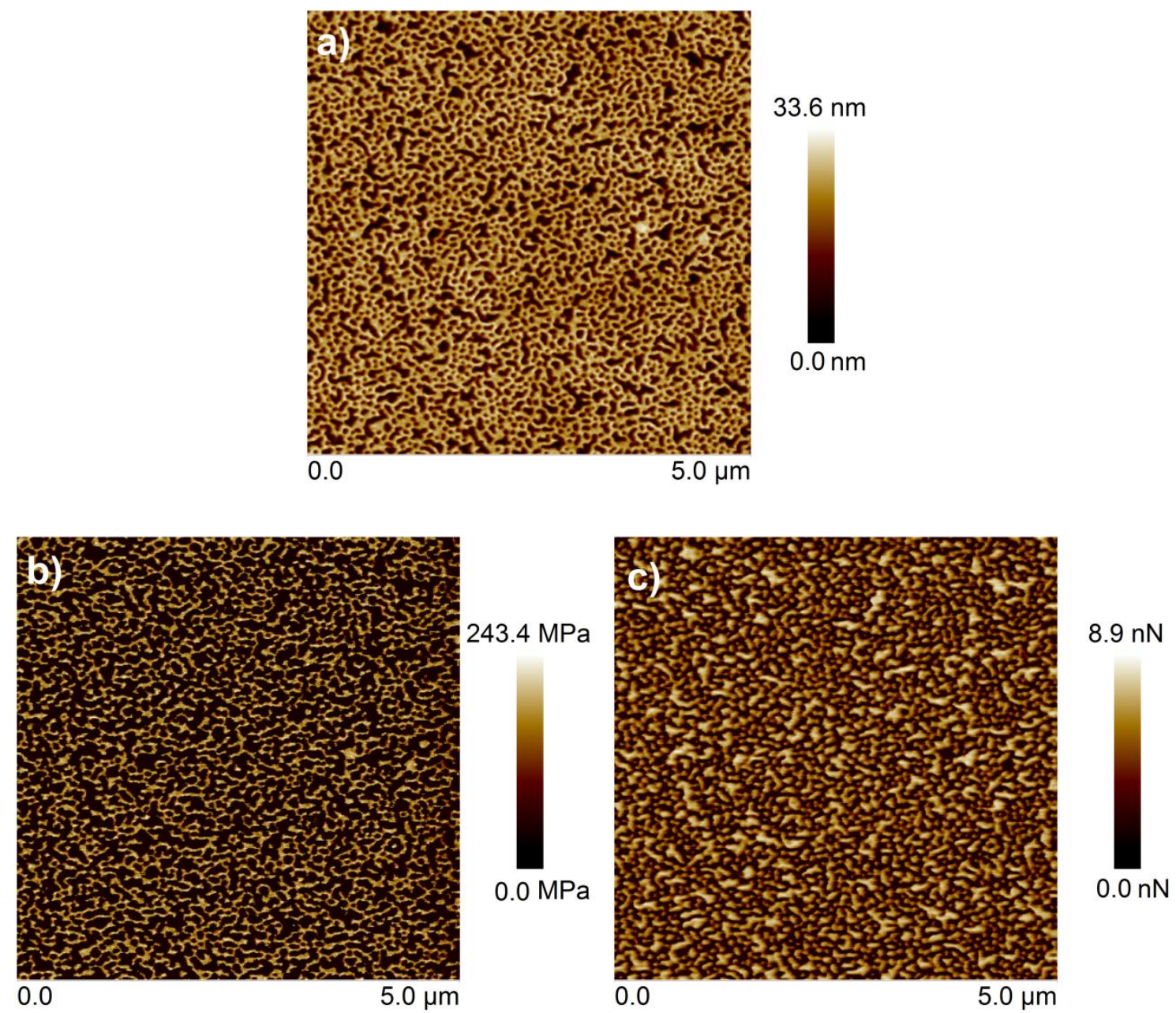

Abbildung 4-44 AFM-Profile für die Probe $\mathbf{V}\left(F_{\mathrm{BA}}=52 \mathrm{~mol} \%\right)$ : a) Höhenprofil; b) DMT-ModulProfil; c) Adhäsionsprofil. Cantilever: FESPA. Auflösung jew. 512 x 512 Pixel. 


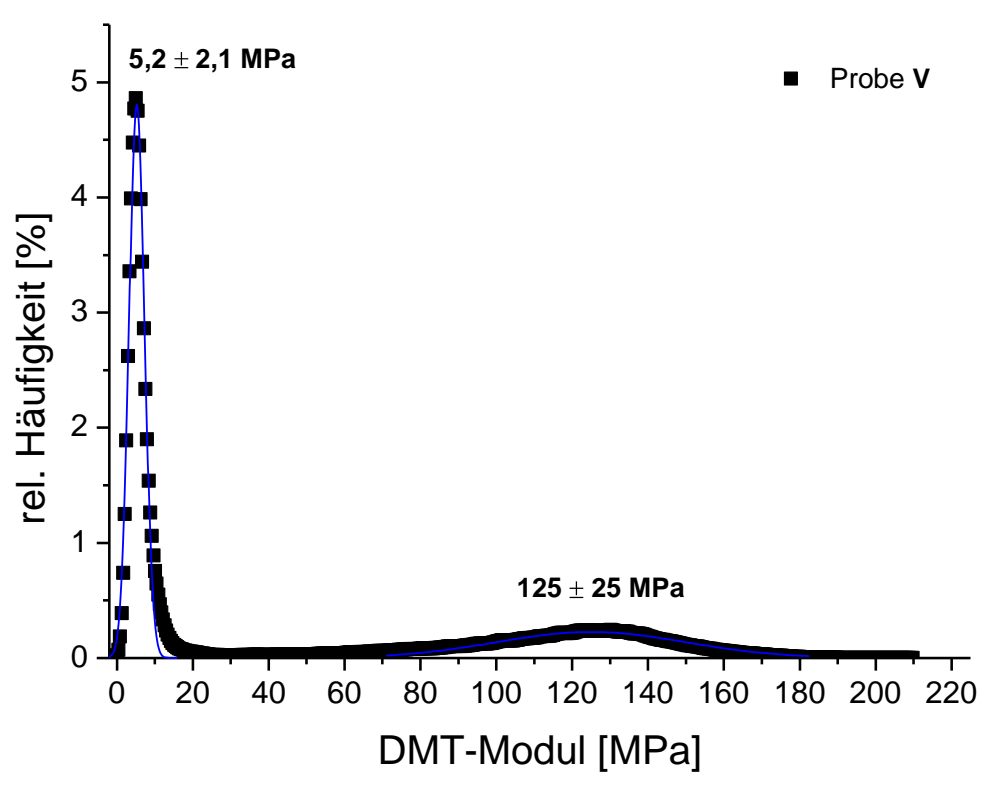

Abbildung 4-45 Häufigkeitsverteilung der DMT-Moduln für die gesamte Probenfläche von Abbildung 4-44 b) für die Probe V. Scanbereich: $3 \mu \mathrm{m}$ x $3 \mu \mathrm{m}$. Die Rohdaten wurden mittel GaußFunktionen (blau) angepasst um einen Mittelwert und die Standardabweichung zu bestimmen.

Es wurden jeweils die aus den Gauß-Verteilungen ermittelten DMT-Moduln $E_{\mathrm{DMT}}$ und als Fehler die Standardabweichung der Gauß-Kurve verwendet. In der Häufigkeitsverteilung kennzeichnet der schmale Peak $(5,2 \pm 2,1 \mathrm{MPa})$ dabei das dunkle Gebiet in Abbildung 4-44 b) und der breite Peak (125 $\pm 25 \mathrm{MPa}$ ) das helle Gitter. Die qualitative Interpretation der erhaltenen Daten zeigt, dass die dunklen Gebiete weicher sind als die hellen Bereiche. Da PMMA einen wesentlich höheren Modul hat als PBA, können durch die DMT-Modul-Bestimmung die dunklen Gebiete dem PBA-Block zugeordnet werden und die hellen Regionen dem P(MMAco-CMA)-Block. Es ist zu erwähnen, dass der Elastizitätsmodul der P(MMA-coCMA)-Domäne im Gegensatz zu dem Elastizitätsmodul reinem PMMAs (ca. $3 \mathrm{GPa}$ ) viel niedriger ist.176 Allerdings muss berücksichtigt werden, dass die Polymerschichten vom PBA, die unterhalb der Oberfläche liegen, wahrscheinlich zu einem niedrigen effektiven Modul des P(MMA-co-CMA)-Blocks führen. Bei dem Messmodus des AFMs muss für die Bestimmung des Moduls die Spitze des Cantilevers in die Probenoberfläche eintauchen, wodurch die unteren Polymerschichten die Messung beeinflussen. Um eine genauere Aussage zu den Moduln der BCP tätigen zu können, wurden der globale Elastizitätsmodul der Probe V mittels DMA bestimmt. Der erhaltene Wert liegt in der Größenordnung der lokalen Elastizitätsmoduln, die mit der PeakForce-QNM ${ }^{\mathrm{T} \mathrm{M}}$-Methode ermittelt wurden. Der globale Elastizitätsmodul für Probe V beträgt ca. $180 \mathrm{MPa}$ und liegt 
damit nicht wie erwartet zwischen den beiden ermittelten lokalen Moduln. Allerdings sind für die Bestimmung der Modulwerte nach der Theorie von Derjaguin, Muller und Toporov gewisse Voraussetzungen nötig. Um den tatsächlichen $E$-Modul zu berechnen, nicht nur den reduzierten Modul $E_{\mathrm{r}}$ (siehe Gleichung (59), Abschnitt 6.6.3), wird die Poissonzahl der Probe $v_{s}$ benötigt, die quadratisch in die Bestimmung des tatsächlichen E-Modul der Probe (siehe Gleichung (36), Abschnitt 2.7.3) eingeht.177 Diese war bei den Proben nicht bekannt und wurde deshalb bei dem voreingestellten Wert von 0,3 belassen. Tatsächlich können die Werte für viskoelastische Materialien im Bereich von 0,3 bis 0,5 liegen, wodurch von einer Abweichung bei den lokalen Moduln ausgegangen werden muss. Die detaillierte Beschreibung zur Kalibrierung des AFMs ist dem Abschnitt 6.6.3 zu entnehmen.

\subsubsection{Verwendung von PeakForce-QNM ${ }^{\mathrm{TM}}$ bei $\mathrm{P}\left(\mathrm{MMA}_{390}-\mathrm{Co}_{\mathrm{C}}-\mathrm{CM} \mathrm{A}_{29}\right)-$ $b$-PBA526}

Die Proben VI und V ähneln sich stark in ihrer Morphologie, die bereits in Abschnitt 4.4.1 genauer diskutiert wurde. Somit wurde bei Probe VI $\left(F_{\mathrm{BA}}=61 \mathrm{~mol} \%\right.$, siehe Tabelle 4-15) ebenfalls vermutet, dass der P(MMA-co-CMA)Block die perforierten Lamellen bildet, die wiederum in einer PBA-Matrix eingebettet sind. Abbildung 4-46 zeigt die unterschiedlichen AFM-Profile der Probe VI.

Das Höhenprofil in Abbildung 4-46 a) zeigt deutlich die in Abschnitt 4.4.1 beschriebene MPS von Probe VI. Das DMT-Modul-Profil [Abbildung 4-46 b)] zeigt für die tiefer liegenden Bereiche einen niedrigen DMT-Modul an, wobei diese Gebiete von einem härteren Bereich (helles Gitter) umgeben sind. Weiterhin zeigt das Adhäsionsprofil in Abbildung 4-46 c), dass die großen klebrigen Bereiche, die hell erscheinen, mehr Adhäsion aufzeigen als das dunkle Gitter. Sowohl das ModulProfil als auch das Adhäsionsprofil lassen eine eindeutige Zuordnung der phasenseparierten Domänen zu den jeweiligen Blöcken zu. Somit kann bestätigt werden, dass die perforierten P(MMA-co-CMA)-Lamellen in einer PBA-Matrix eingebettet sind. Zusätzlich konnten die DMT-Moduln der jeweiligen Blöcke bestimmt werden. Abbildung 4-47 stellt die Rohdaten der DMT-Modulwerte der Oberfläche und die jeweils angepasste Gaußkurve für Probe VI dar. 

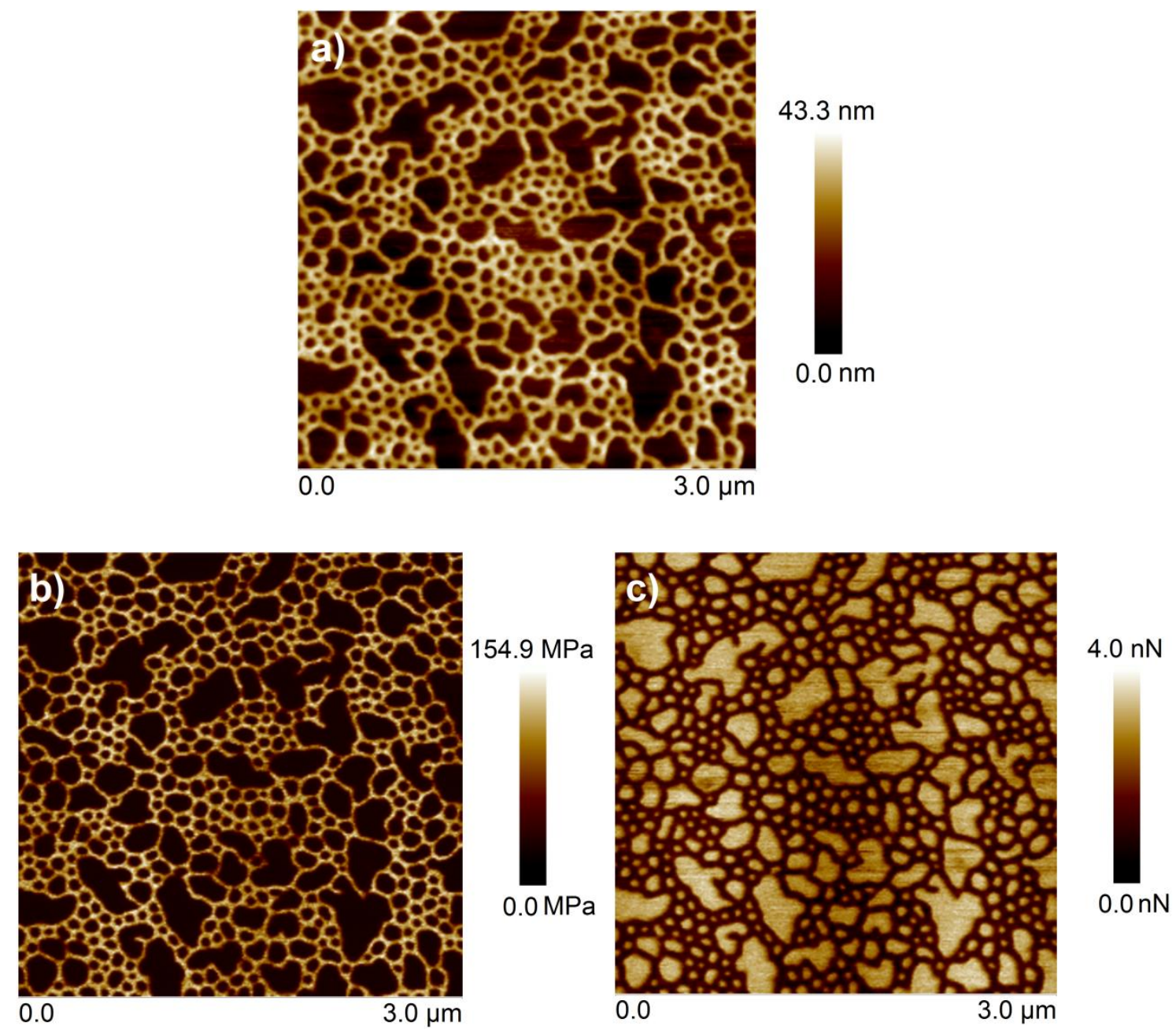

Abbildung 4-46 AFM-Profile für die Probe VI ( $\left.F_{\mathrm{BA}}=61 \mathrm{~mol} \%\right)$; a) Höhenprofil; b) DMT-ModulProfil; c) Adhäsionsprofil. Cantilever: FESPA. Auflösung jew. 512 x 512 Pixel.

Der signifikante Peak bei 6,7 MPa kennzeichnet den dunklen Hintergrund in Abbildung 4-46 b) und der breite Peak bei 86 MPa das helle Gitter. Die Zuordnung der lokalen Moduln zu den jeweiligen Blöcken erfolgt wie in dem Unterkapitel zuvor. Somit repräsentieren die in dem Höhenprofil tiefer gelegenen Bereiche den PBA-Block und die höheren Domänen den P(MMA-co-CMA)-Block. Der mit der DMA ermittelte globale Modul für die Probe VI liegt bei ca. $60 \mathrm{MPa}$. Er liegt somit zwischen den zwei Werten und veranschaulicht den erwarteten Durchschnitt der beiden lokalen Moduln. 


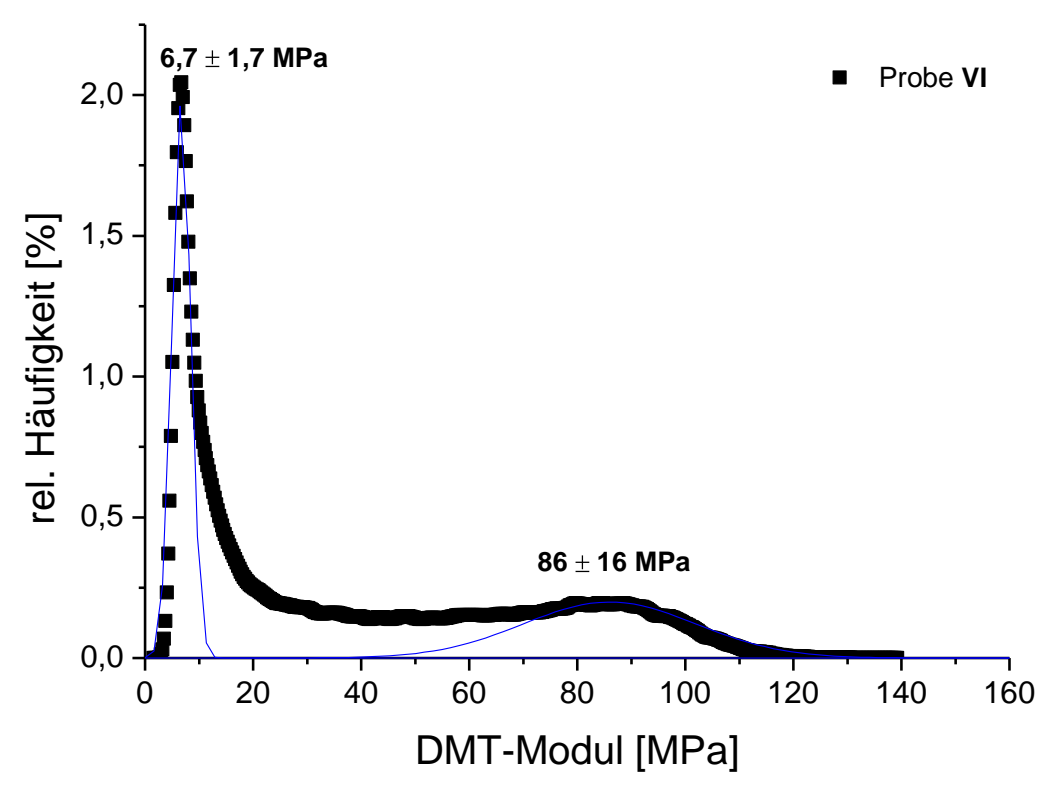

Abbildung 4-47 Häufigkeitsverteilung der DMT-Moduln für die gesamte Probenfläche von Abbildung 4-46 b) für die Probe VI. Scanbereich: $3 \mu \mathrm{m}$ x $3 \mu \mathrm{m}$. Die Rohdaten wurden mittel GaußFunktionen angepasst um einen Mittelwert und die Standardabweichung zu bestimmen.

\subsubsection{Verwendung von PeakForce-QNM ${ }^{\mathrm{TM}}$ bei P(MMA95-co-CMA9)-b- PBA229}

Die bemerkenswerte Morphologie von Probe VII $\left(F_{\mathrm{BA}}=68 \mathrm{~mol} \%\right.$, siehe Tabelle 4-15) wird nochmal in dem AFM-Höhenprofil in Abbildung 4-48 gezeigt, wobei die mäanderförmige Struktur bereits in Abschnitt 4.4.1 ausführlich diskutiert wurde. Das Höhenprofil veranschaulicht den Phasenwechsel zwischen den höher gelegenen Zylindern, die in dem AFM-Bild hell dargestellt werden und den dazwischen liegenden tieferen Bereichen, die in dem Profil dunkel gekennzeichnet sind und im Folgenden als Täler bezeichnet werden.

In dem folgenden Unterkapitel sollen die mechanischen Eigenschaften für diese Probe bestimmt werden, wobei zu der Durchführung der Messung zu sagen ist, dass für die Bestimmung des Elastizitätsmoduls bei dem angewendeten PeakForce-QNM ${ }^{\mathrm{Tm}}$-Modus der Cantilever tiefer in die Probenoberfläche eintauchen muss. Bei der Betrachtung des Höhenprofils (Abbildung 4-48) fiel auf, dass die waagerecht ausgerichteten Täler (grüner Kasten in Abbildung 4-48), die zwischen den Zylindern liegen, tiefer und breiter wirken als die senkrecht ausgerichteten Täler (roter Kasten in Abbildung 4-48). 


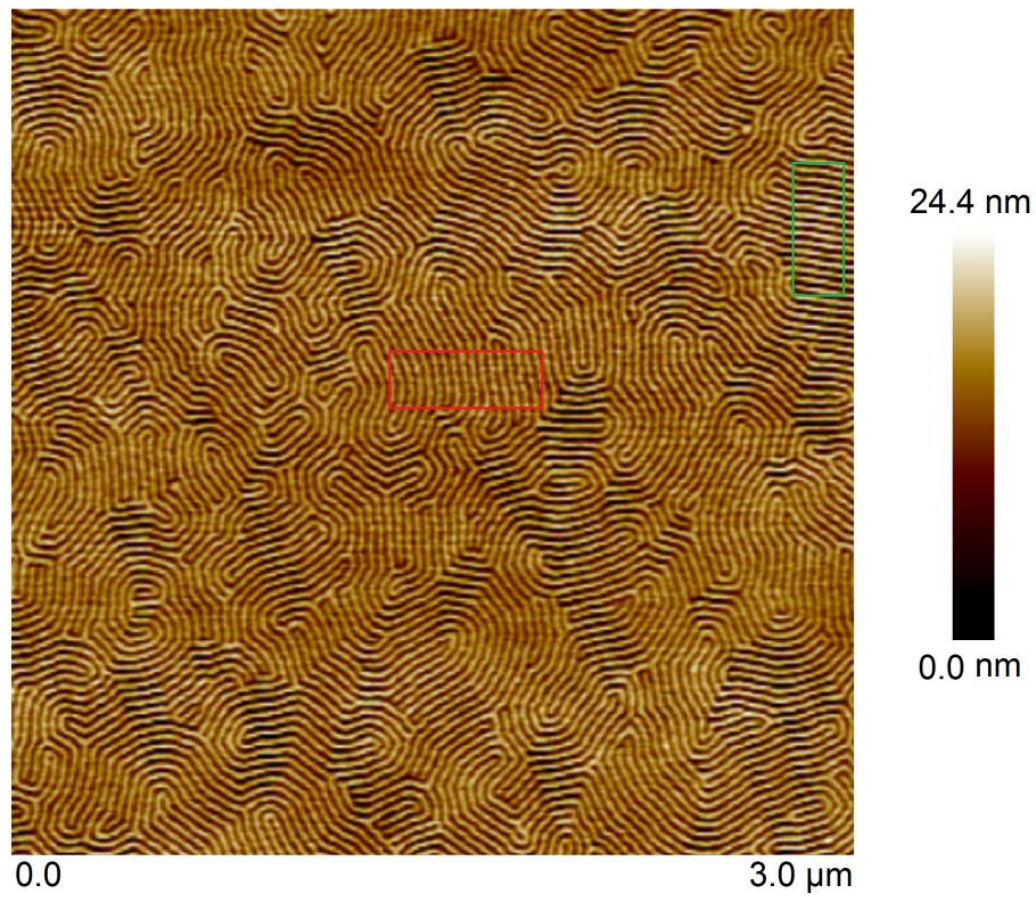

Abbildung 4-48 Vergrößerter Ausschnitt (3 $4 \mathrm{~m} \times 3 \mu \mathrm{m})$ des AFM-Höhenprofils von Probe VII $\left(F_{\mathrm{BA}}=68 \mathrm{~mol} \%\right)$. Der grüne Rahmen kennzeichnet die waagerecht ausgerichteten Domänen und der rote Rahmen die senkrecht ausgerichteten. PF setpoint automatisch gesetzt. Scanbereich: $5 \mu \mathrm{m}$ x $5 \mu \mathrm{m}$. Cantilever: RFESP. Auflösung 512 x 512 Pixel.

Dieses optische Phänomen liegt vermutlich daran, dass die waagerechten Täler in der schnellen, waagerechten Messrichtung liegen (siehe Abschnitt 6.6.3). Bei dieser Scanrichtung bewegt sich der Cantilever in waagerechter Richtung über die Probenoberfläche und dabei drückt die Spitze des Cantilevers am Startpunkt des Tals zwischen die Zylinder und schiebt diese dabei auseinander. Setzt die Spitze ihren Weg in waagerechter Richtung fort, so kann sie tiefer eindringen, da die Zylinder etwas weiter voneinander entfernt sind. Trifft die Cantileverspitze nun auf die senkrecht ausgerichteten Strukturen, drückt die Spitze bei fortsetzender Messrichtung abwechselnd auf einen Zylinder und ein Tal, wodurch die Täler nicht stark aufgeweitet werden und die Spitze nicht so tief eindringen kann. Normalerweise wird dieses Messproblem mit Nutzung eines geringeren PF setpoints behoben. Allerdings wird für die Bestimmung des DMT-Moduls eine ausreichende Eindringtiefe in die Probenoberfläche (hoher PF setpoint) benötigt.

Abbildung 4-49 zeigt das DMT-Modul-Profil von Probe VII. Insgesamt wird deutlich, dass die Zylinder einen höheren DMT-Modul aufweisen als die dazwischen liegenden Täler. Somit können die Zylinder dem P(MMA-co-CMA)Block zugeordnet werden und die Täler der PBA-Phase, wodurch eine Verschiebung der härteren Zylinder in der weichen Matrix sinnvoll erscheint. Das 
zuvor beschriebene Messproblem wird auch in Abbildung 4-49 deutlich, da die DMT-Moduln der waagerecht ausgerichteten Zylinder im Gegensatz zu den senkrecht ausgerichteten einen höheren Wert besitzen. Um eine quantitative Aussage zum DMT-Modul treffen zu können, wurde ein Verfahren von Raaij et al. übernommen und auf die Zylinderstruktur von Probe VII angewendet.178 Dabei wurden in dem DMT-Modul-Profil (Abbildung 4-49) stichprobenartig Querschnitte durch die waagerecht (grün) sowie senkrecht (rot) ausgerichteten Zylinder gelegt. In Abbildung 4-49 sind exemplarisch zwei dieser Querschnitte $(1 ; 2)$ gekennzeichnet.

Abbildung 4-50 zeigt die Auftragung der Rohdaten der DMT-Moduln für die zwei gekennzeichneten Bereiche aus dem DMT-Profil (Abbildung 4-49). Die Maxima repräsentieren dabei die jeweiligen $\mathrm{P}(\mathrm{MMA}-\mathrm{co}-\mathrm{CMA})$-Blöcke (Zylinder) und die Minima die PBA-Phase (Täler). Tabelle 4-24 fasst die ermittelten mittleren DMTModuln $\bar{E}_{\mathrm{DMT}}$ für die Blöcke mit der jeweiligen Standardabweichung zusammen, wobei der DMT-Modul $E_{\mathrm{DMT}, 1}$ den P(MMA-co-CMA)-Block kennzeichnet und $E_{\mathrm{DMT}, 2}$ den PBA-Block.

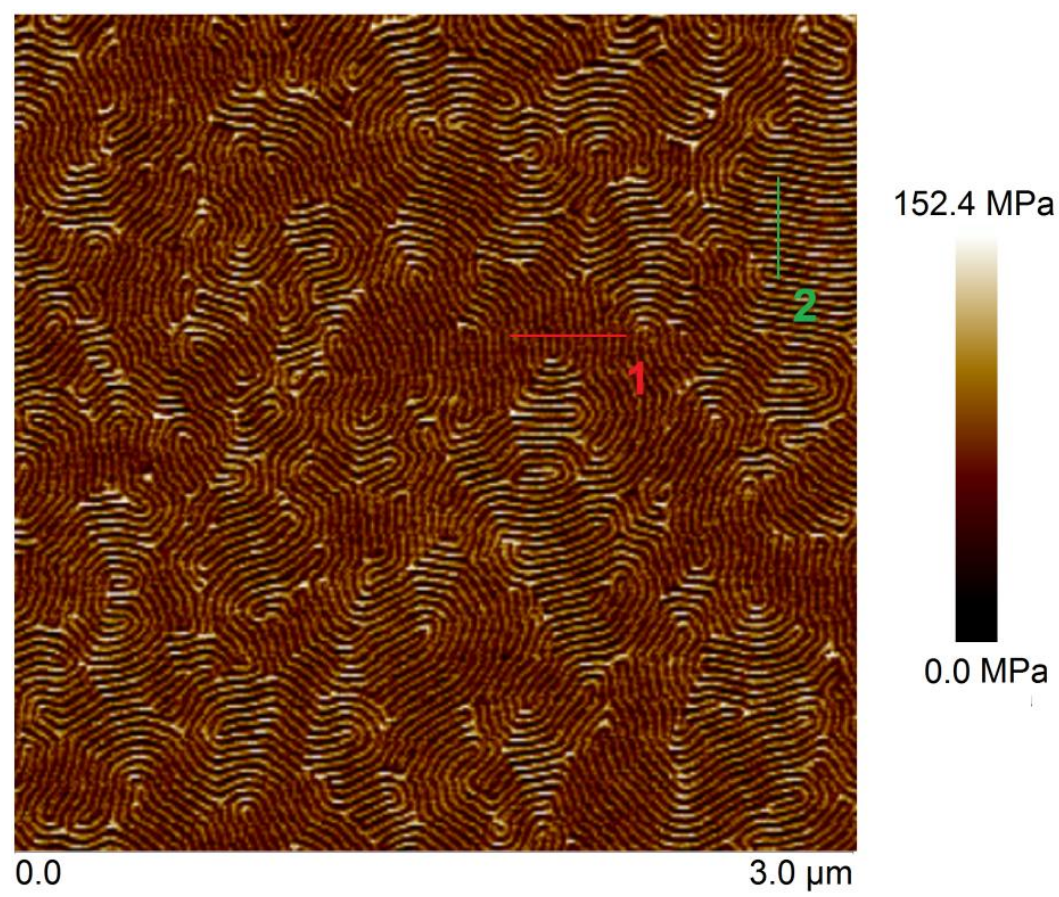

Abbildung 4-49 Vergrößerter Ausschnitt ( $3 \mu \mathrm{m} \times 3 \mu \mathrm{m}$ ) des DMT-Modul-Profils von Probe VII. Der rote Querschnitt (1) kennzeichnet senkrecht und der grüne Querschnitt (2) die waagerecht ausgerichteten Zylinder. Scanbereich: $5 \mu \mathrm{m} \times 5 \mu \mathrm{m}$. PF setpoint automatisch gesetzt. Cantilever: REFESP. Auflösung jew. 512 x 512 Pixel. 


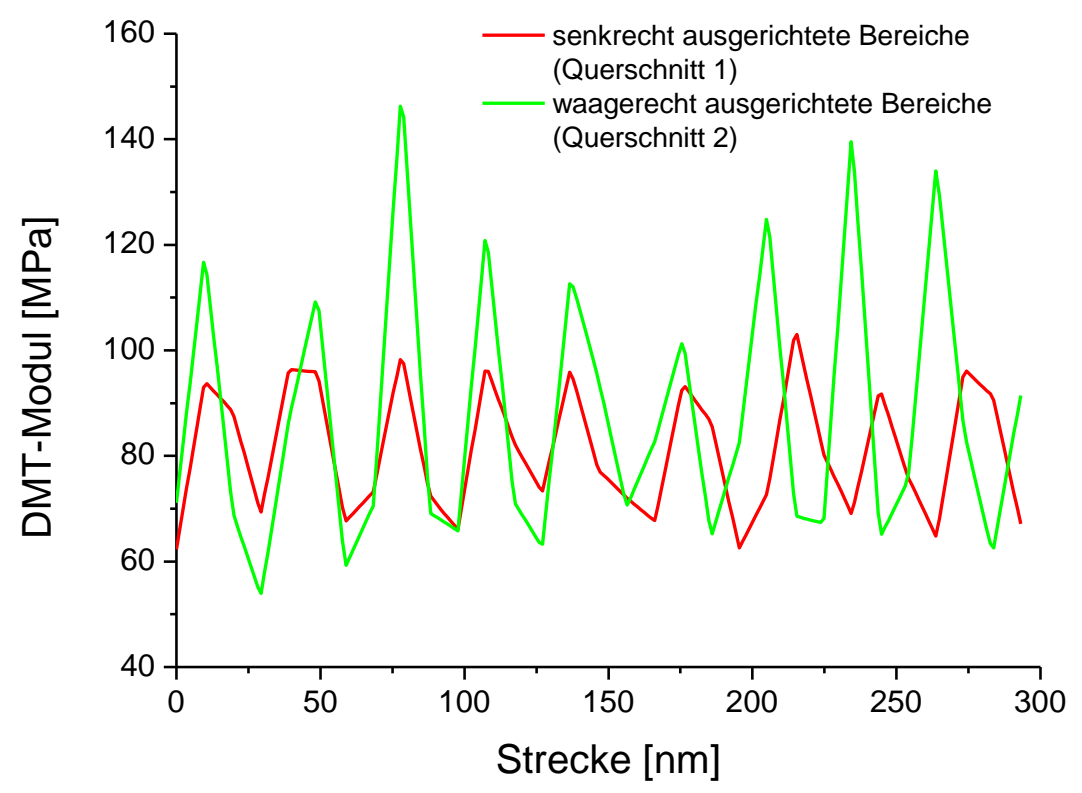

Abbildung 4-50 Auftragung der DMT-Moduln für die Querschnitte 1 (rot) und 2 (grün) der Probe VII in Abbildung 4-49. Die Maxima kennzeichnen die jeweiligen P(MMA-co-CMA)-Zylinder und die Minima die PBA-Täler.

Tabelle 4-24 Mittelwerte der DMT-Moduln für die jeweiligen Blöcke des BCPs VII in waagerechter und senkrechter Ausrichtung, wobei der mittlere DMT-Modul $\bar{E}_{\mathrm{DMT}, 1}$ den P(MMA-co-CMA)-Block kennzeichnet und $\bar{E}_{\mathrm{DMT}, 2}$ den PBA-Block repräsentiert

\begin{tabular}{|l|l|l|}
\hline Blockausrichtung & $\overline{\boldsymbol{E}}_{\text {DMT,1 }}$ [MPa] & $\overline{\boldsymbol{E}}_{\text {DMT,2 }}$ [MPa] \\
\hline waagerecht & $122,8 \pm 14,8$ & $64,4 \pm 5,1$ \\
\hline senkrecht & $93 \pm 9,6$ & $67 \pm 3,4$ \\
\hline
\end{tabular}

Insgesamt kann eine durch die Blockausrichtung hervorgerufene Abweichung für die mittleren DMT-Moduln der unterschiedlichen Blockkomponenten festgestellt werden. Dieser Effekt kann auf die oben beschriebene Messrichtung zurückgeführt werden. Die Probe VII zeigt eine dicht gepackte Zylinderstruktur, bei der die jeweiligen Blöcke eng beieinander liegen. Der Radius der Cantileverspitze (R $\sim 8 \mathrm{~nm}$ ) begrenzt die Auflösung des AFMs und je näher die Blöcke nebeneinander liegen, desto schwerer wird eine Messung der separaten Phasen (siehe Abschnitt 6.6.3) und die Wahrscheinlichkeit für die Messung überlappender Phasen steigt. Bei den DMT-Moduln der waagerecht ausgerichteten Blöcke liegt eine größere Differenz zwischen den P(MMA-co-CMA)- und PBA-Modulwerten vor als bei den senkrecht ausgerichteten Blöcken. Bei der waagerechten Scanrichtung der Cantileverspitze kommt es bei den senkrecht ausgerichteten Blöcken zu einem ständigem Wechsel zwischen den Phasen, wohingegen bei den waagerecht 
ausgerichteten Blöcken die jeweilige Homophase über eine längere Distanz gemessen werden kann. Dadurch werden die mittleren DMT-Moduln für die waagerecht ausgerichteten Blöcke als die aussagekräftigeren Werte angesehen.

Zusätzlich wurden die DMT-Modulwerte für die gesamte Probenoberfläche des DMT-Profils bestimmt (Abbildung 4-49). Die Rohdaten der DMT-Modulwerte der Oberfläche und die jeweils angepasste Gaußkurve für Probe VII sind in Abbildung 4-51 dargestellt.

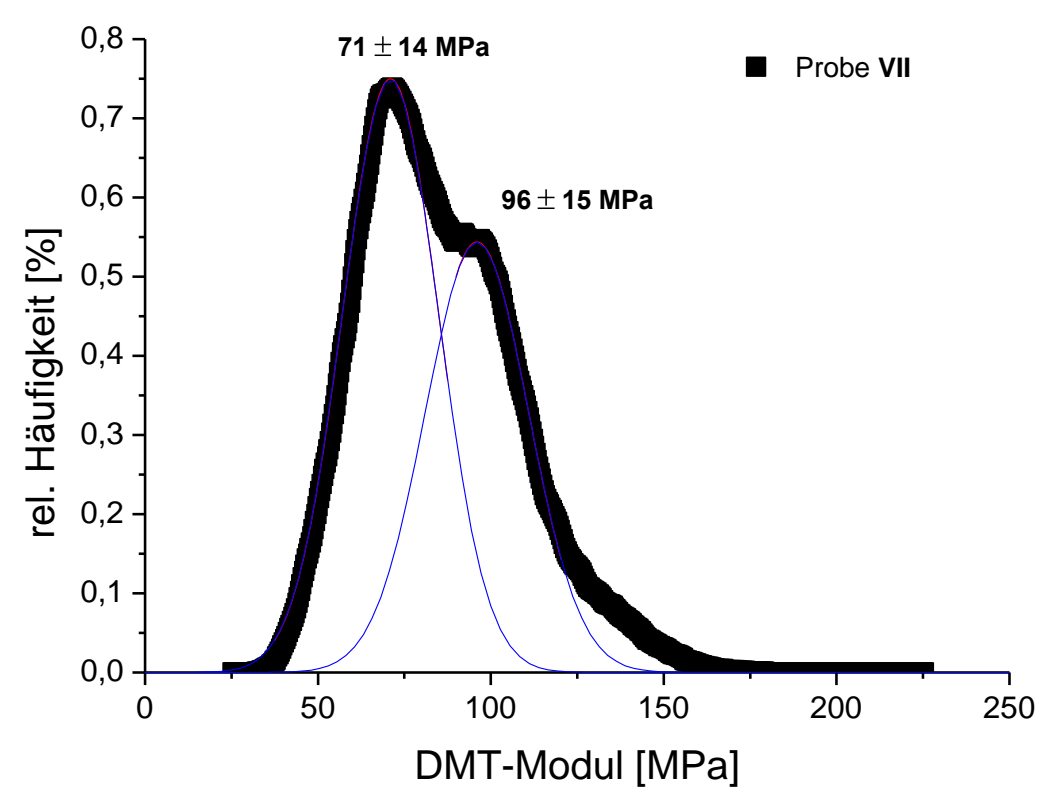

Abbildung 4-51 Häufigkeitsverteilung der DMT-Moduln für die gesamte Probenfläche von Abbildung 4-49 für die Probe VII. Scanbereich: $5 \mu \mathrm{m} \times 5 \mu \mathrm{m}$. Die Rohdaten wurden mittel GaußFunktionen angepasst um einen Mittelwert und die Standardabweichung zu bestimmen.

Es wurden jeweils die aus den Gauß-Verteilungen ermittelten DMT-Moduln $E_{\text {DMT }}$ verwendet und als Fehler die Standardabweichung der Gauß-Kurve verwendet. In der Häufigkeitsverteilung kennzeichnet der Peak bei $71 \pm 14 \mathrm{MPa}$ die tiefer gelegene PBA-Phase und der Peak bei $96 \pm 15$ MPa den P(MMA-co-CMA)-Block. Da für die Bestimmung der beiden DMT-Modulwerte (96 MPa, $71 \mathrm{MPa}$ ) die gesamte Probenoberfläche in Abbildung 4-49 verwendet wurde und nicht wie davor zwei Querschnitte einer gewählten Strecke, repräsentieren die DMT-Moduln aussagekräftige Mittelwerte für die Probe VII. Trotz alledem muss festgehalten werden, dass durch die oben beschriebenen Messprobleme (Spitzenradius, Scanrichtung) die DMT-Modul-Werte für die Probe VII mit ihrer dicht gepackten Zylinderstruktur insgesamt nur als Näherungswerte zu betrachten sind. 


\subsubsection{Fazit}

In diesem Abschnitt wurden die lokalen DMT-Moduln der einzelnen Blöcke für drei unterschiedliche Proben des Blockcopolymersystems P(MMA-co-CMA)- $b$-PBA mittels AFM bestimmt. Die ermittelten DMT-Moduln der Proben V, VI und VII werden in der folgenden Tabelle zusammengefasst, wobei der DMT-Modul $E_{\mathrm{DMT}, 1}$ den P(MMA-co-CMA)-Block kennzeichnet und $E_{\mathrm{DMT}, 2}$ den PBA-Block repräsentiert.

Tabelle 4-25 Übersicht der DMT-Moduln $E_{\mathrm{DMT}}$ für die Proben V, VI und VII des BCPs P(MMA-co-CMA)- $b$-PBA.

\begin{tabular}{|l|l|l|l|}
\hline Probe & $\begin{array}{l}\boldsymbol{F}_{\text {BA }} \\
{[\mathbf{m o l} \%]}\end{array}$ & $\begin{array}{l}\boldsymbol{E}_{\text {DMT, }} \\
{[\mathbf{M P a}]}\end{array}$ & $\begin{array}{l}\boldsymbol{E}_{\text {DMT,2 }} \\
{[\mathbf{M P a}]}\end{array}$ \\
\hline V & 54 & $125 \pm 25$ & $5,2 \pm 2,1$ \\
\hline VI & 63 & $86 \pm 16$ & $6,7 \pm 1,7$ \\
\hline VII & 70 & $96 \pm 15$ & $71 \pm 14$ \\
\hline
\end{tabular}

Die Größenordnung der DMT-Moduln für die P(MMA-co-CMA)-Blöcke besitzt für alle Proben eine ähnliche Tendenz, wobei die Abweichung von dem Modulwert reinen PMMAs durch die Messmethode erklärt werden konnte. Der DMT-Modul des PBA-Blocks von Probe VII hat im Vergleich zu den anderen Proben einen höheren Wert, was wiederum durch die Messprobleme bzw. dicht gepackte Zylinderanordnung zustande kommt. Da bei den Proben V und VI größere PBABereiche vermessen werden konnten, werden diese DMT-Moduln-Werte als die aussagekräftigeren Werte angesehen. Das AFM ermöglicht die Bestimmung der lokalen DMT-Moduln der einzelnen phasenseparierten Bereiche und somit deren Zuordnung $\mathrm{zu}$ den jeweiligen Blöcken des Polymersystems. Allerdings muss berücksichtigt werden, dass es sich dabei um keine Absolutwerte handelt und sie lediglich eine Tendenz aufzeigen können. 


\subsection{UV-induzierte Dimerisierungsreaktion und Dimerspaltung}

Die in den ersten Block P(MMA-co-CMA) eingebauten photo-vernetzbaren Coumarin-Einheiten können eine reversible Photodimerisierung eingehen (siehe Abschnitt 2.8.2). Die Vernetzungen können die gebildeten polymeren Mikrostrukturen stabilisieren und die mechanischen Eigenschaften des Polymersystems verändern. Von besonderem Interesse ist dabei, dass in der vorliegenden Arbeit alle Vernetzungsprozesse im festen Zustand des Systems durchgeführt wurden, wohingegen in der Literatur vorwiegend Vernetzungsreaktionen des Coumarins in Lösung bekannt sind. ${ }^{161,179}$ Der UV-induzierte Dimerisierungsprozess des BCPMaterials erfolgte bei unterschiedlichen Wellenlängen und bei verschiedenen Temperaturen. Die reversible Photodimerisierung wird in Schema 4-2 veranschaulicht. Die rotbraune Polymerkette stellt den PBA-Block und die gelbe Kette den P(MMA-co-CMA)-Block dar.
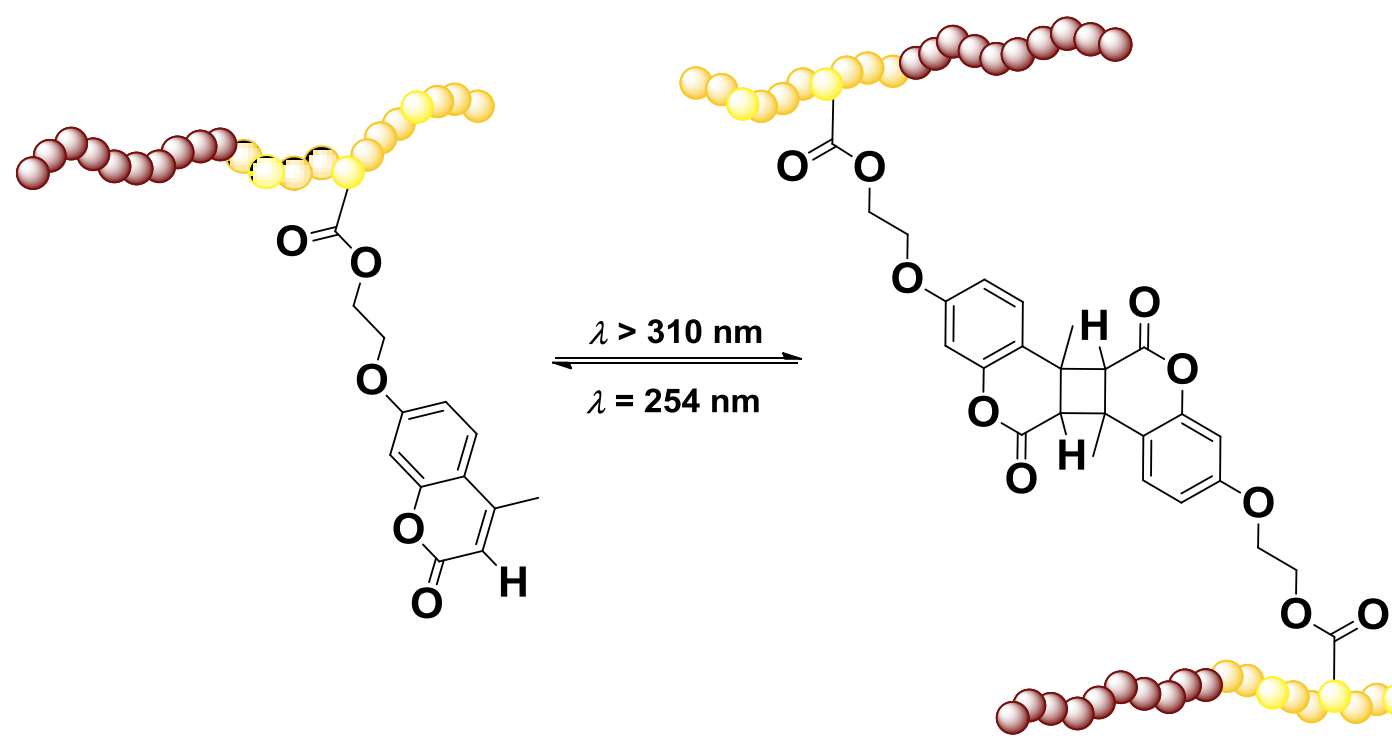

Schema 4-2 Reversible photochemische Dimerisierung der CMA-Einheiten in den synthetisierten BCP, wobei die rotbraune Polymerkette den PBA-Block und die gelbe Kette den P(MMA-co-CMA)Block darstellt.

In den folgenden Unterkapiteln werden die Dimerisierung und die Dimerspaltung näher erläutert. Beide Reaktionsschritte wurden mit UV/Vis-Spektroskopie und AFM analysiert. Weiterhin wurden mit Hilfe der DMA die Auswirkungen der Bestrahlung von $\lambda>310 \mathrm{~nm}$ auf die mechanischen Eigenschaften der BCP untersucht. 


\subsubsection{Dimerisierung der Coumarin-Einheiten des BCPs P(MMA-co- CMA)-b-PBA}

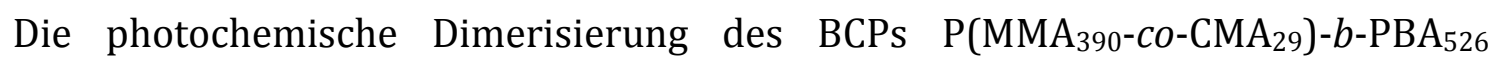
(Probe VI) wurde mit Hilfe von UV/Vis-Spektroskopie analysiert. Dafür wurden dünne Polymerfilme mit Schichtdicken von 1,8 bis 3,4 $\mu$ m hergestellt und bei zwei unterschiedlichen Temperaturen mit UV-Licht der Wellenlänge $\lambda>310 \mathrm{~nm}$ bestrahlt. Die detaillierte Probenpräparation ist dem Abschnitt 6.6.4 zu entnehmen. Zuerst wurde die Vernetzung der im P(MMA-co-CMA)-Block befindlichen Coumarin-Einheiten bei einer Bestrahlung von $\lambda>310 \mathrm{~nm}$ und einer Temperatur von $21^{\circ} \mathrm{C}$ (Raumtemperatur, RT) untersucht. In Abbildung 4-52 ist eine Abnahme der Absorbanz der Coumarin-Doppelbindung (siehe Schema 4-2) bei $\lambda=317 \mathrm{~nm}$ mit zunehmender Bestrahlungszeit $\mathrm{zu}$ erkennen, wodurch ein erfolgreicher Vernetzungsprozess verdeutlicht wird.
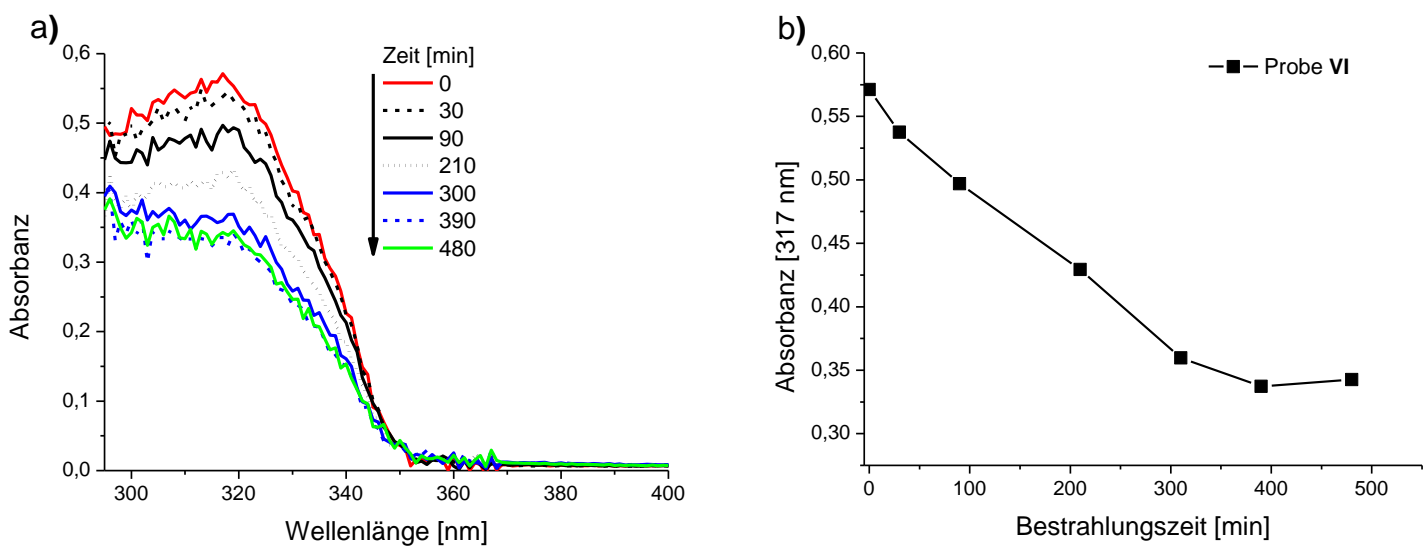

Abbildung 4-52 a) zeitabhängiges UV/Vis-Spektrum von Probe VI bei einer Bestrahlung mit UVLicht der Wellenlänge $\lambda>310 \mathrm{~nm}$ bei $T=21^{\circ} \mathrm{C}$; b) zeitabhängige Entwicklung der Absorptionsbande bei $317 \mathrm{~nm}$.

Der Vernetzungsprozess der Coumarin-Einheiten wurde bei einer Temperatur unterhalb der $T_{\mathrm{g}}$ des P(MMA-co-CMA)-Blocks durchgeführt. Aufgrund der fehlenden Kettenbeweglichkeit können nicht alle CMA-Einheiten eine Vernetzung eingehen. Um eine ausreichende Beweglichkeit der CMA-Einheiten garantieren zu können, wurde ein Polymerfilm der Probe VI bei einer Temperatur oberhalb ihrer $T_{\mathrm{g}}\left(T=125^{\circ} \mathrm{C}\right)$ bestrahlt. Für einen Vergleich der Vernetzungsprozesse für beide Temperaturen $\left(21^{\circ} \mathrm{C}\right.$ und $\left.125^{\circ} \mathrm{C}\right)$ wurde mit Hilfe der folgenden Gleichung der Grad der Photodimersierung (PD) bestimmt: 


$$
\operatorname{PD}[\%]=\frac{\left(A_{0}-A_{t}\right)}{A_{0}} \cdot 100
$$

wobei $A_{0}$ die Absorption der Coumarin-Einheiten vor der UV-Bestrahlung $(t=0)$ darstellt und $A_{t}$ die Absorption nach der jeweiligen Bestrahlungszeit $t$ ist. Abbildung 4-53 zeigt die Auftragung des Photodimerisierungsgrades gegen die Bestrahlungszeit für die Vernetzungsprozesse bei den jeweiligen Temperaturen.

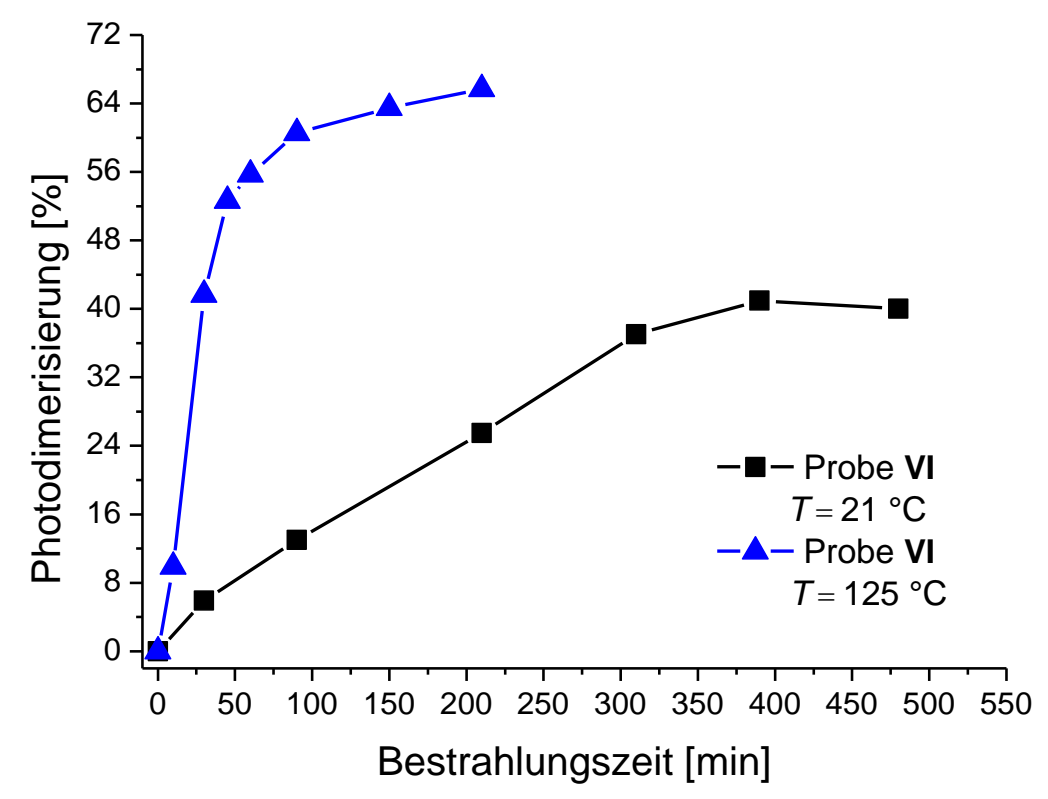

Abbildung 4-53 Anstieg des Photodimerisierungsgrades für dünne Polymerfilme der Probe VI mit zunehmender Bestrahlungszeit $(\lambda>310 \mathrm{~nm})$ bei $T=21^{\circ} \mathrm{C}$ und $125^{\circ} \mathrm{C}$.

Mit der Erhöhung der Kettenbeweglichkeit (Abbildung 4-53, $T=125^{\circ} \mathrm{C}$ ) wird innerhalb kurzer Bestrahlungszeit $(t=210 \mathrm{~min}$ ) ein Photodimerisierunggrad von $66 \%$ erreicht. Wohingegen bei einer eingeschränkten Beweglichkeit (Abbildung $4-53, T=21^{\circ} \mathrm{C}$ ) nach einer wesentlich längeren Bestrahlungszeit ( $t=500 \mathrm{~min}$ ) ein geringerer Photodimerisierungsgrad (PD $=40 \%$ ) erreicht wurde. Da die Moleküle in einer Polymerlösung die bestmögliche Beweglichkeit besitzen, wurde das BCP (Probe VI) in THF gelöst $\left(c=2,85 \cdot 10^{-7} \mathrm{~mol} / \mathrm{L}\right)$. Das Ergebnis der UV/Vis-Messung für das gelöste BCP wird in Abbildung 4-54 gezeigt. 


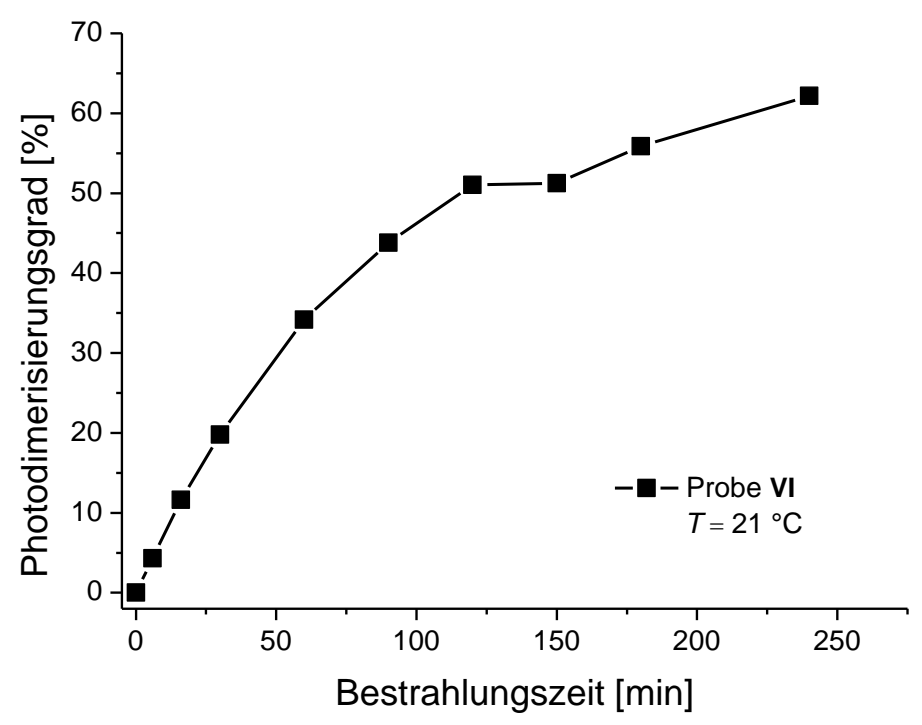

Abbildung 4-54 Entwicklung des Photodimerisierungsgrades für die Probe VI in THF mit zunehmender Bestrahlungszeit.

Für die Polymerlösung wurde nach einer Bestrahlungszeit von $t=240 \mathrm{~min}$ ein Vernetzungsgrad von $62 \%$ ermittelt. Im Vergleich zur Polymerlösung konnte für dünne Polymerfilme ein ebenso effizienter Vernetzungsprozess ermittelt werden. Allerdings muss für eine effiziente Photodimerisierung von Polymerfilmen eine ausreichende Kettenbeweglichkeit der jeweiligen Blöcke und somit der CoumarinEinheiten gegeben sein (Bestrahlung oberhalb der $T_{\mathrm{g}}, 125^{\circ} \mathrm{C}$ ).

Der Vernetzungsprozess wurde zusätzlich mit dem AFM untersucht. Dafür wurden dünne Polymerfilme des BCPs P(MMA $\left.{ }_{390}-c o-\mathrm{CMA}_{29}\right)-b$-PBA 26 (Probe VI) mit UVLicht der Wellenlänge $\lambda>310 \mathrm{~nm}$ bestrahlt (die Probenpräparation ist dem Abschnitt 6.6.3 zu entnehmen). Die Vernetzung wurde bei Temperaturen von $21^{\circ} \mathrm{C}$ und $125^{\circ} \mathrm{C}$ durchgeführt. Danach wurden die Proben bei $160^{\circ} \mathrm{C}$ über Nacht im Vakuumofen getempert. Abbildung 4-55 zeigt die AFM-Ergebnisse der behandelten Proben vor und nach der Bestrahlung sowie dem Tempern. In Abbildung 4-55 ist zusätzlich eine Referenzprobe dargestellt, die ohne Bestrahlung über Nacht bei $160{ }^{\circ} \mathrm{C}$ getempert wurde. 

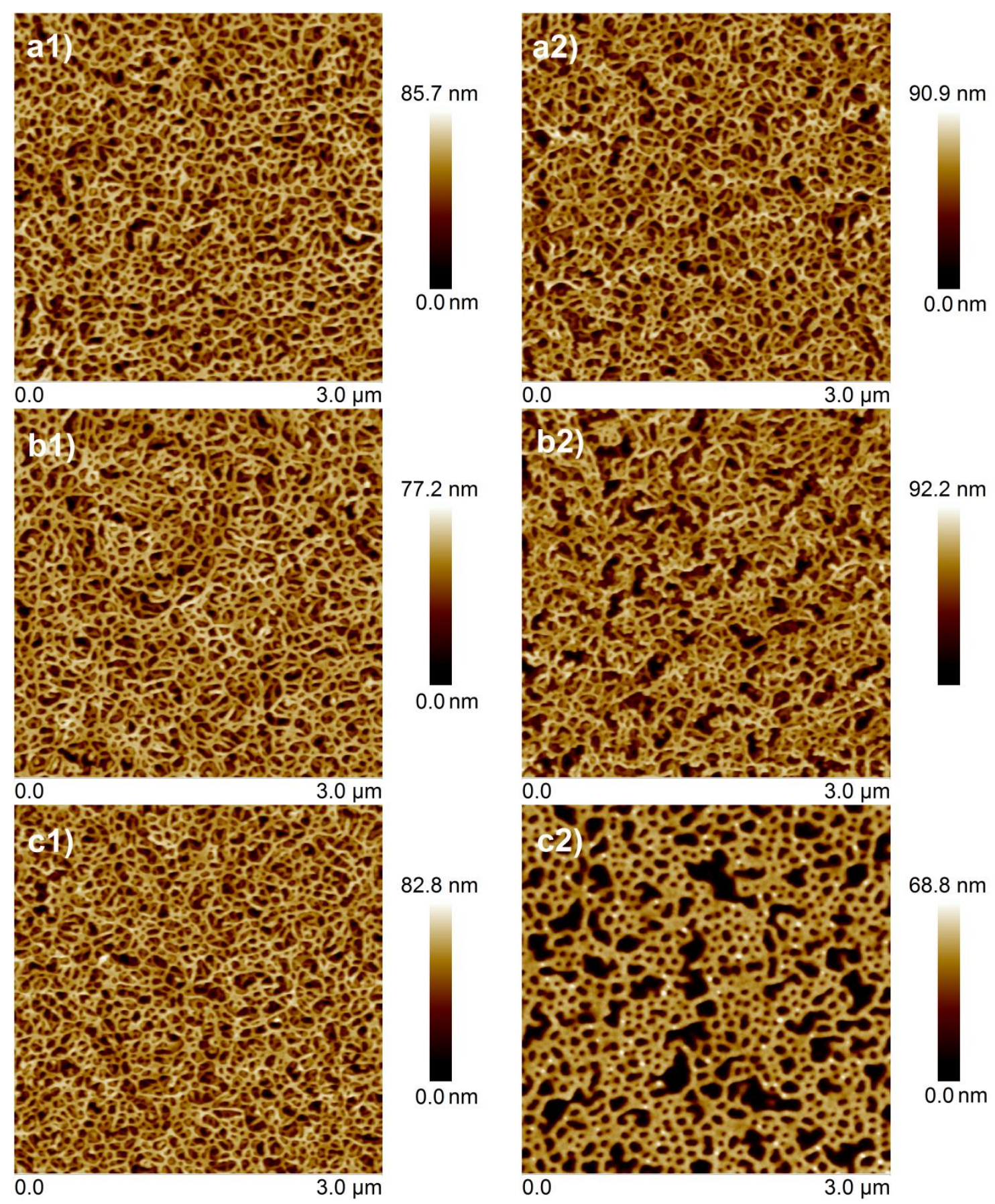

Abbildung 4-55 AFM Höhenprofile der Probe VI: a1) per spincoating erhaltene Ausgangsprobe; a2) nach $4 \mathrm{~h}$ Bestrahlung $(\lambda>310 \mathrm{~nm})$ bei $T=21^{\circ} \mathrm{C}$ und Tempern bei $160^{\circ} \mathrm{C}$ über Nacht; b1) gespincoatete Ausgangsprobe; b2) nach $4 \mathrm{~h}$ Bestrahlung $(\lambda>310 \mathrm{~nm})$ bei $T=125^{\circ} \mathrm{C}$ und Tempern bei $160^{\circ} \mathrm{C}$ über Nacht; $\mathrm{c} 1$ ) per spincoating erhaltene Ausgangsprobe; c2) ohne Bestrahlung und nach Tempern bei $160^{\circ} \mathrm{C}$ über Nacht.

Die Abbildung 4-55a2) und b2) verdeutlichen eine erfolgreiche Photodimerisierung im BCP P(MMA-co-CMA) $37-b$ - $\mathrm{PBA}_{63}$ (Probe VI). Die Vernetzungen zwischen den Polymerketten erschweren es dem BCP-System, die Gleichgewichts- 
morphologie während des Temperns auszubilden. Somit bleibt die ungeordnete Struktur der per spincoating erhaltenen Ausgangsproben [a1) und b1)] nach dem Tempern erhalten. Die AFM-Ergebnisse verdeutlichen, dass bereits eine geringe Anzahl von Coumarin-Einheiten einen deutlichen Einfluss auf das BCP-System besitzt. Obwohl bei der Vernetzung bei $21^{\circ} \mathrm{C}$ ein niedriger Photodimerisierungsgrad festgestellt wurde, reicht die geringe Anzahl an vernetzenden CMA-Einheiten aus, um das Polymersystem an der Ausbildung der Gleichgewichtsmorphologie zu hindern. Die Probe in Abbildung 4-55 b2) wurde im Gegensatz zu a2) zuerst auf $125^{\circ} \mathrm{C}$ aufgeheizt und nach $10 \mathrm{~min}$ mit UV-Licht bestrahlt. Die Kettenbeweglichkeit in Probe b2) wird mit zunehmender Temperatur größer, wodurch das Material versucht die Gleichgewichtsmorphologie (große tiefe Bereiche) auszubilden. Allerdings wird dem Polymersystem nicht genügend Zeit zur Strukturanordnung gegeben, da die Bestrahlung der Probe nach 10 min bei $125^{\circ} \mathrm{C}$ erfolgte. Die Referenzprobe in Abbildung 4-55 c2) zeigt nach dem Temperprozess die in Abschnitt 4.4.1 beschriebene Gleichgewichtsmorphologie, die sich durch große, tiefe Bereiche bestehend aus der PBA-Phase an der Oberfläche auszeichnet.

Während des Temperns der Referenzprobe kann das BCP-System im thermodynamischen Gleichgewichtszustand eine geordnete, phasenseparierte Struktur ausbilden. Die Umstrukturierung des BCPs wird in den AFM-Profilen durch die signifikante Höhenabnahme von 82,8 auf 68,8 nm erkennbar [Abbildung 4-55 c1) und c2)]. Wohingegen bei den vernetzten BCP-Systemen [Abbildung 4-55 a2) und b2)] eine Höhenzunahme nach dem Temperprozess erkennbar ist. Die P(MMA-coCMA)-Phase wird durch die Vernetzungen daran gehindert, die Gleichgewichtsmorphologie während des Temperns auszubilden. Die PBA-Blöcke ordnen sich zu den, für die Probe VI typischen, großen Bereiche an, wodurch es zu einer Anhebung der P(MMA-co-CMA)-Phase und somit zu einer Höhenzunahme im AFMProfil kommen kann. Dieser Effekt erscheint besonders ausgeprägt in Abbildung 4-55 b2), da die PBA-Phase in der Probe schon während des Aufheizens auf $125^{\circ} \mathrm{C}$ Zeit zur Umstrukturierung hatte. Insgesamt wurde bei der Probe in Abbildung 4-55 b2) eine Zunahme der Höhe von 77,2 auf 92,2 nm festgestellt.

Die Vernetzungen haben einen zusätzlichen Einfluss auf die mechanischen Eigenschaften: Wird das Polymersystem durch Veränderung der Temperatur oder durch das Anlegen einer Kraft aus dem Gleichgewichtszustand gedrängt, ist eine Relaxation nur eingeschränkt möglich. Durch die Vernetzungen kommt es zu topologischen Einschränkungen des Systems und die Frustration zwischen der 
Netzwerk-Konnektivität und der Unverträglichkeit der Blockkomponenten wirkt sich auf die mechanischen Eigenschaften aus. Der Einfluss der Photodimerisierung auf die mechanischen Eigenschaften des BCPs P(MMA-co-CMA)- $b$-PBA (Proben $\mathbf{V}$ und VI) wurde mittels DMA untersucht. Die globalen Elastizitätsmoduln für die Proben V und VI wurden nach jedem Bestrahlungsvorgang $(\lambda>310 \mathrm{~nm})$ bei $T=21{ }^{\circ} \mathrm{C}(\mathrm{RT})$ mit Hilfe der DMA ermittelt. Abbildung 4-56 zeigt den Verlauf der Elastizitätsmoduln in Abhängigkeit der Bestrahlungszeit.

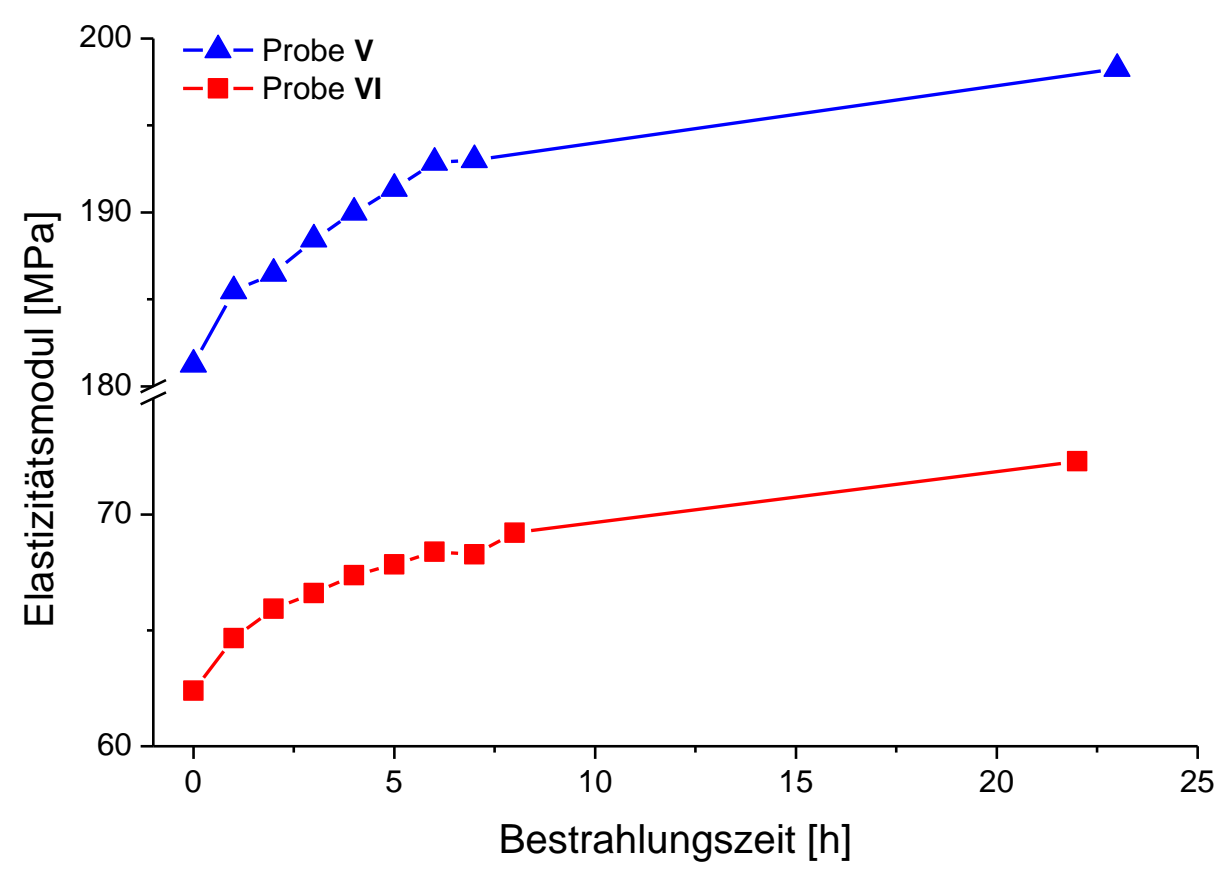

Abbildung 4-56 Entwicklung der Elastizitätsmoduln für dünne Polymerfolien der Proben V und VI in Abhängigkeit von der Bestrahlungszeit $(\lambda>310 \mathrm{~nm})$.

Die Proben V und VI werden mit zunehmender Bestrahlungszeit und steigender Vernetzungsanzahl härter (10-15\%), wodurch eine erfolgreiche photochemische Dimerisierung festgestellt werden kann. Die Zunahme des absoluten E-Moduls ist bei der Probe mit dem höheren P(MMA-co-CMA)-Anteil (V) ausgeprägter als bei der Probe mit niedrigerem (VI). Dieser Effekt erklärt sich dadurch, dass die CMAEinheiten in dem PMMA-Block eingebaut sind. Somit besitzen Proben mit einem größeren PMMA-Anteil eine größere Menge an Vernetzern.

\section{Fazit}

Insgesamt wurde bei der Bestrahlung des BCP-Systems P(MMA-co-CMA)- $b$-PBA mit UV-Licht der Wellenlänge $\lambda>310 \mathrm{~nm}$ eine erfolgreiche photochemische Dimerisierung der Coumarin-Einheiten festgestellt. Die AFM-Ergebnisse ver- 
deutlichen, dass die Vernetzung eines geringen CMA-Anteils einen großen Einfluss auf die Morphologie des Polymersystems hat. In der vorliegenden Arbeit wurden alle Vernetzungsprozesse im festen Zustand des Systems durchgeführt und bei der Bestrahlung der Proben unterhalb der $T_{\mathrm{g}}$ des P(MMA-co-CMA)-Blocks wurde eine hohe Effizienz für die Dimerisierung der CMA-Einheiten festgestellt. Die unkomplizierte Durchführbarkeit der photochemischen Dimerisierung des Coumarin-Derivats stellt neue und einfache Wege für Material-Design und Modifikation von BCP bereit.

\subsubsection{Dimerspaltung der Coumarin-Einheiten des BCPs P(MMA- co-CMA)-b-PBA}

Die photochemische Dimerspaltung des BCPs P(MMA $\left.{ }_{390}-\mathrm{Co}-\mathrm{CMA}_{29}\right)-b-\mathrm{PBA}_{526}$ (Probe VI) wurde mit Hilfe von UV/Vis-Spektroskopie untersucht. Dafür wurden die vernetzten Proben aus Abschnitt 4.7.1 bei zwei Temperaturen $\left(21^{\circ} \mathrm{C}\right.$ und $125^{\circ} \mathrm{C}$ ) mit UV-Licht der Wellenlänge $\lambda=254 \mathrm{~nm}$ bestrahlt. Wie im Verlauf dieser Arbeit bereits erwähnt, ist der Anstieg der Absorbanz bei $\lambda=317 \mathrm{~nm}$ auf den Aufbau der Coumarin-Doppelbindung in Folge einer Dimerspaltung zurückzuführen (siehe Schema 4-2). Abbildung 4-57 zeigt das zeitabhängige UV/VisSpektrum für Probe VI bei $T=21^{\circ} \mathrm{C}$ und die Zunahme der für die CoumarinDoppelbindung charakteristischen Absorptionsbande bei $317 \mathrm{~nm}$.
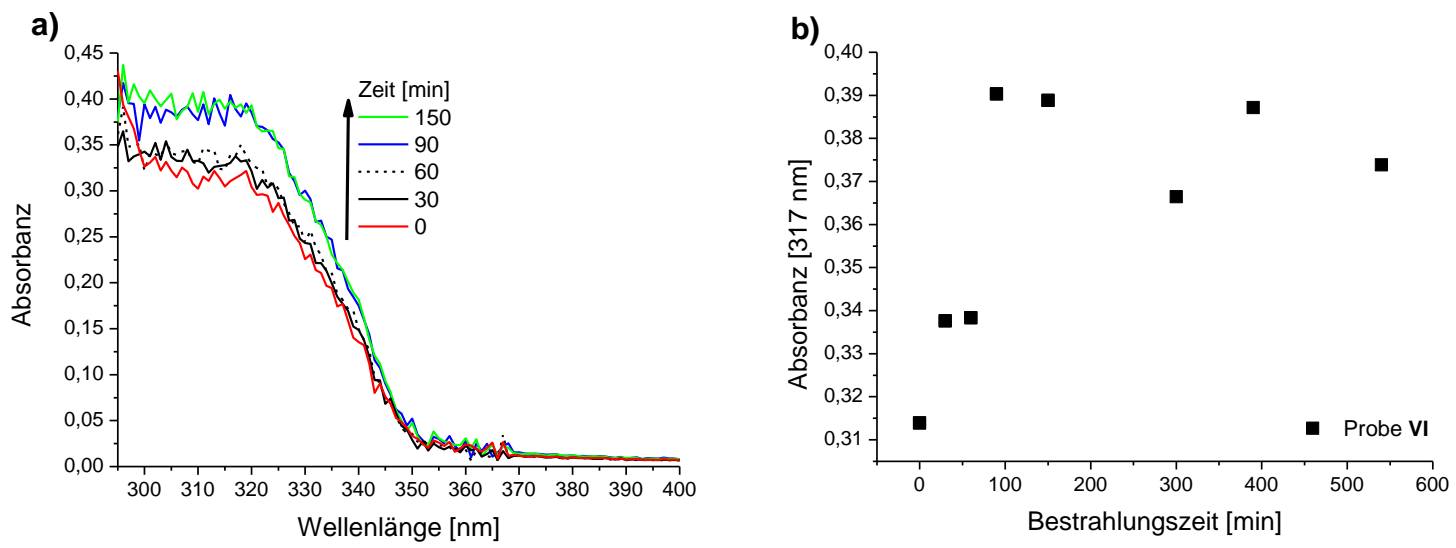

Abbildung 4-57 a) zeitabhängiges UV/Vis-Spektrum von Probe VI bei einer Bestrahlung mit UVLicht der Wellenlänge $\lambda=254 \mathrm{~nm}$ bei $T=21^{\circ} \mathrm{C}$; b) zeitabhängige Entwicklung der Absorptionsbande bei $317 \mathrm{~nm}$.

Abbildung 4-57 stellt eine erfolgreiche Dimerspaltung bei $T=21^{\circ} \mathrm{C}$ dar. Mit Gleichung (43) aus Abschnitt 4.7.1 wurde zusätzlich der Photodimerisierungsgrad der Reaktion bestimmt. In Abbildung 4-58 werden die Spaltungsprozesse für eine 
Bestrahlung mit UV-Licht der Wellenlänge $\lambda=254 \mathrm{~nm}$ bei einer Temperatur von $21^{\circ} \mathrm{C}$ und $125^{\circ} \mathrm{C}$ verglichen. Dafür wurde in Abbildung 4-58 der Grad der Photodimerisierung für beide Temperaturen in Abhängigkeit von der Bestrahlungszeit aufgetragen.

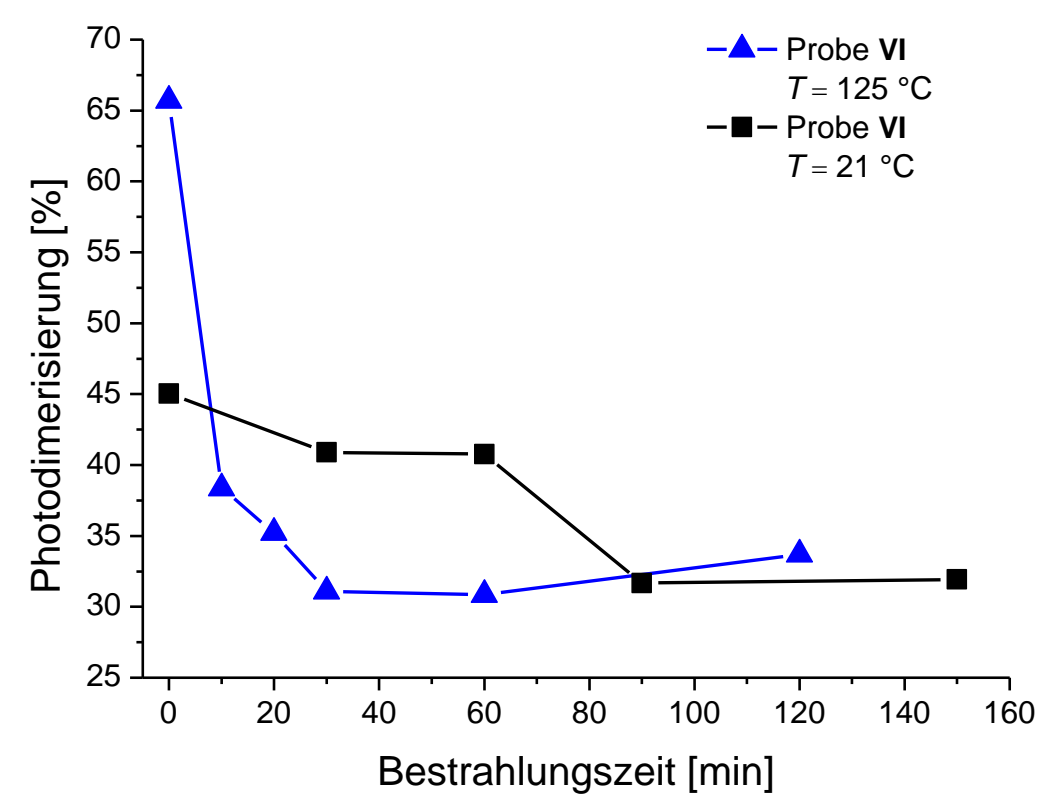

Abbildung 4-58 Entwicklung des Photodimerisierungsgrades für dünne Polymerfilme der Probe VI mit zunehmender Bestrahlungszeit $(\lambda=254 \mathrm{~nm})$ bei $T=21^{\circ} \mathrm{C}$ und $125^{\circ} \mathrm{C}$. Die unterschiedlichen Ausgangspunkte für die Dimerspaltung resultieren aus den Endwerten der Vernetzungsreaktion beider Proben (siehe Abbildung 4-53).

Bei einer erhöhten Kettenbeweglichkeit (Abbildung 4-58, $T=125^{\circ} \mathrm{C}$ ) konnte der Photodimerisierungsgrad innerhalb kurzer Bestrahlungsdauer ( $t=30 \mathrm{~min}$ ) von $66 \%$ auf ca. $31 \%$ herabgesetzt werden. Der Dimerisierungsgrad für den Polymerfilm VI bei $T=21^{\circ} \mathrm{C}$ (Abbildung 4-58) konnte nach einer Bestrahlungsdauer von $t=90 \mathrm{~min}$ um ca. $13 \%$ verringert werden. Im Vergleich zur Probe VI bei $125^{\circ} \mathrm{C}$ besitzt der bei RT vernetzte Polymerfilm (VI) weniger vernetzte CoumarinEinheiten, woraus sich ein geringerer Grad für die Dimerspaltung ( 13\%) ergibt. Insgesamt konnte für beide Spaltungsreaktionen keine vollständige Dimerspaltung erreicht werden. Neben der photochemischen Analyse der Polymerfilme wurde die Dimerspaltung zusätzlich in einer Polymerlösung (Probe VI) untersucht. Das Ergebnis der UV/Vis-Messung für das gelöste BCP $\left(c=2,85 \cdot 10^{-7} \mathrm{~mol} / \mathrm{L}\right)$ wird in Abbildung 4-59 gezeigt. 


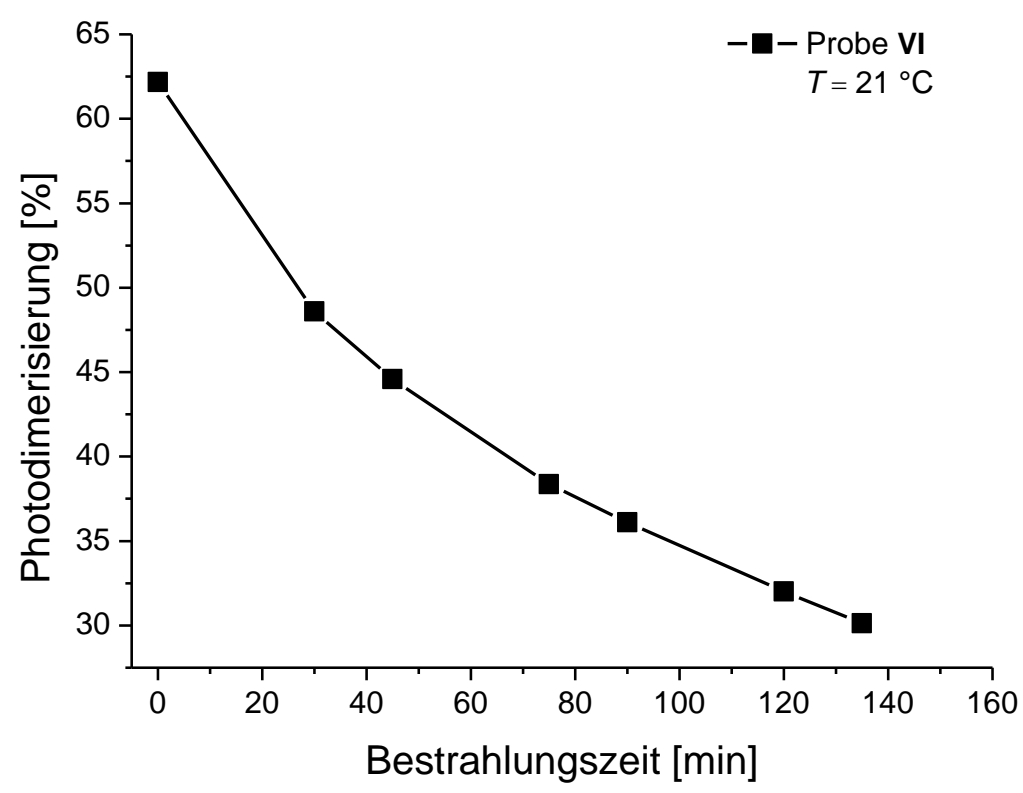

Abbildung 4-59 Entwicklung des Photodimerisierungsgrades für die Probe VI in THF mit zunehmender Bestrahlungszeit.

In Abbildung 4-59 wurde für die in THF gelöste Probe VI eine Abnahme des Photodimerisierungsgrades von ca. $62 \%$ auf $31 \%$ mit zunehmender Bestrahlungsdauer festgestellt. Folglich konnte für die UV-Bestrahlung $(\lambda=254 \mathrm{~nm})$ des im festen und flüssigen Zustand vorliegenden BCPs (VI) keine vollständige Dimerspaltung erreicht werden. Der für die UV-Bestrahlung der Probe VI verwendete Bandpassfilter besitzt bei der Wellenlänge $\lambda=254 \mathrm{~nm}$ eine Transmission von ca. $78 \%$, die durch die zusätzliche Verwendung eines Lichtleiters herabgesetzt wird. Ursachen für die unvollständige Dimerspaltung der Coumarin-Einheiten können zum einen die zu geringe Transmission bei einer Wellenlänge von $\lambda=254 \mathrm{~nm}$ sein und zum anderen können bei der Dimerspaltung durch die hohe Energie der UV-Strahlung bei $\lambda=254 \mathrm{~nm}$ Nebenreaktionen stattfinden (siehe Abschnitt 2.8.2).

Weiterhin wurde der Spaltungsprozess für die mit dem AFM untersuchten Polymerfilme aus Abschnitt 4.7.1 analysiert. Dafür wurden die Proben nach der Vernetzung [Abbildung 4-60a2) und b2)] mit UV-Licht der Wellenlänge $\lambda=254 \mathrm{~nm}$ bei $21^{\circ} \mathrm{C}$ und $125^{\circ} \mathrm{C}$ bestrahlt und im Anschluss bei $160^{\circ} \mathrm{C}$ über Nacht im Vakuumofen getempert. Abbildung 4-60 zeigt die vernetzten AFM-Proben aus dem Abschnitt 4.7.1 und die Ergebnisse der Dimerspaltung. 

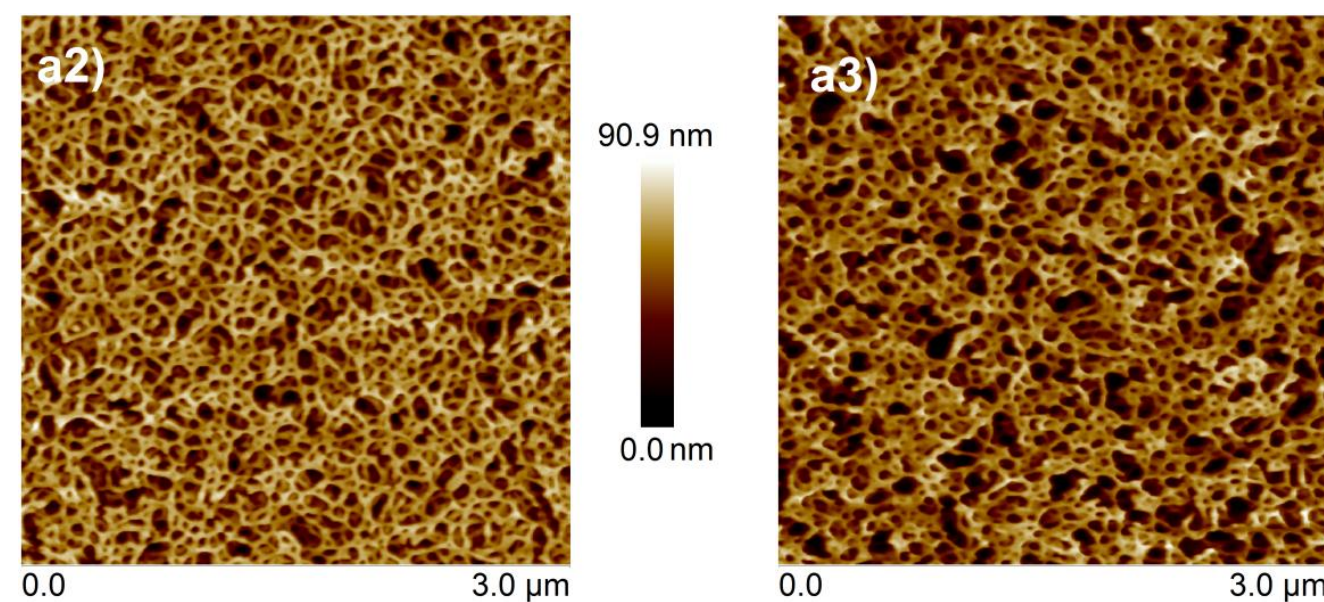

$82.8 \mathrm{~nm}$

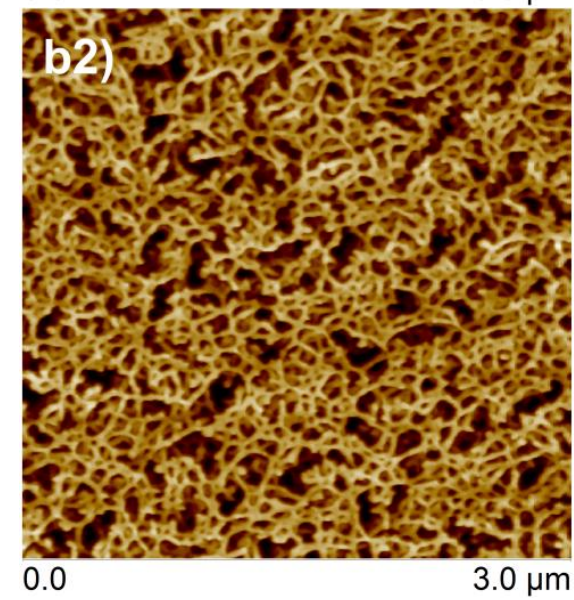

$92.2 \mathrm{~nm}$

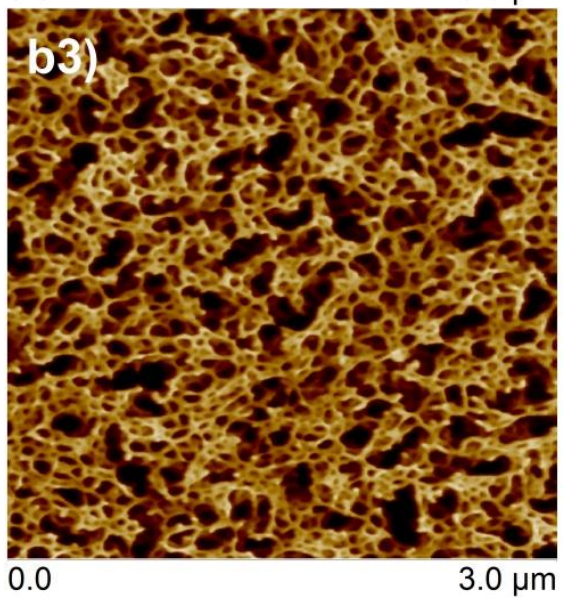

$79.7 \mathrm{~nm}$

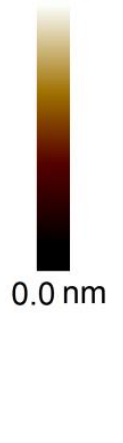

0.0

$3.0 \mu \mathrm{m}$

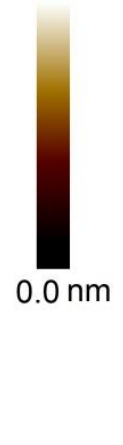

Abbildung 4-60 AFM-Höhenprofile der Probe VI: a2) nach $4 \mathrm{~h}$ Bestrahlung $(\lambda>310 \mathrm{~nm})$ bei $T=21^{\circ} \mathrm{C}$ und Tempern bei $160^{\circ} \mathrm{C}$ über Nacht; a3) nach $4 \mathrm{~h}$ Bestrahlung $(\lambda=254 \mathrm{~nm})$ bei $T=21^{\circ} \mathrm{C}$ und Tempern bei $160^{\circ} \mathrm{C}$ über Nacht; b2) nach $4 \mathrm{~h}$ Bestrahlung $(\lambda>310 \mathrm{~nm})$ bei $T=125^{\circ} \mathrm{C}$ und Tempern bei $160^{\circ} \mathrm{C}$ über Nacht; b3) nach $4 \mathrm{~h}$ Bestrahlung $(\lambda=254 \mathrm{~nm})$ bei $T=125^{\circ} \mathrm{C}$ und Tempern bei $160^{\circ} \mathrm{C}$ über Nacht.

Insgesamt wurde in den Abbildung 4-60 a3) und b3) für beide Polymerfilme eine Höhenabnahme des Systems festgestellt. Nach der Dimerspaltung wird das System nicht länger in seiner Struktur festgehalten und die Umordnung der Blöcke nach dem Tempern wird durch die Vergrößerung der PBA-Bereiche (Abbildung 4-60 a3) und b3), tiefe Gebiete) verdeutlicht. Allerdings wurde mit den UV/VisErgebnissen eine unvollständige Dimerspaltung der Coumarin-Einheiten nachgewiesen. Folglich findet bei dem Polymersystem eine Annäherung an die Gleichgewichtsmorphologie statt und die vollständige MPS kann nicht erreicht werden [siehe Referenzprobe in Abbildung 4-55 c2)]. 


\subsection{Ermittlung des Ordnungs-Unordnungs-Übergangs}

Der Ordnungs-Unordnungs-Übergang (engl.: order-disorder transition, ODT) der bisher untersuchten BCP (siehe Abschnitt 4.4) liegt bei zu hohen Temperaturen, um ihn nachweisen zu können, da sowohl die niedrige Zersetzungstemperatur des Polymers sowie die technischen Einschränkungen beim AFM, wie z. B. die Nutzung der Heizzelle bis max. $250^{\circ} \mathrm{C}$, die Bestimmung des ODTs verhindern. Allerdings zeigt die DMA-Untersuchung von Probe $\mathbf{X}$ einen Hinweis auf den ODT bei einer Temperatur von ca. $140{ }^{\circ} \mathrm{C}$ (siehe Abschnitt 4.3). In dem folgenden Abschnitt wird die Struktur und das Phasenverhalten von Probe $\mathbf{X}$ mittels AFM untersucht.

Das AFM-Höhenprofil in Abbildung 4-61 veranschaulicht die Gleichgewichtsmorphologie von Probe $\mathbf{X}$, die eine Anordnung von liegenden P(MMA-co-CMA)Zylindern in einer darunterliegenden PBA-Matrix zeigt. Der Vergleich der MPS von Probe $\mathbf{X}$ mit den anderen Blockcopolymeren erfolgte in Abschnitt 4.4.1.

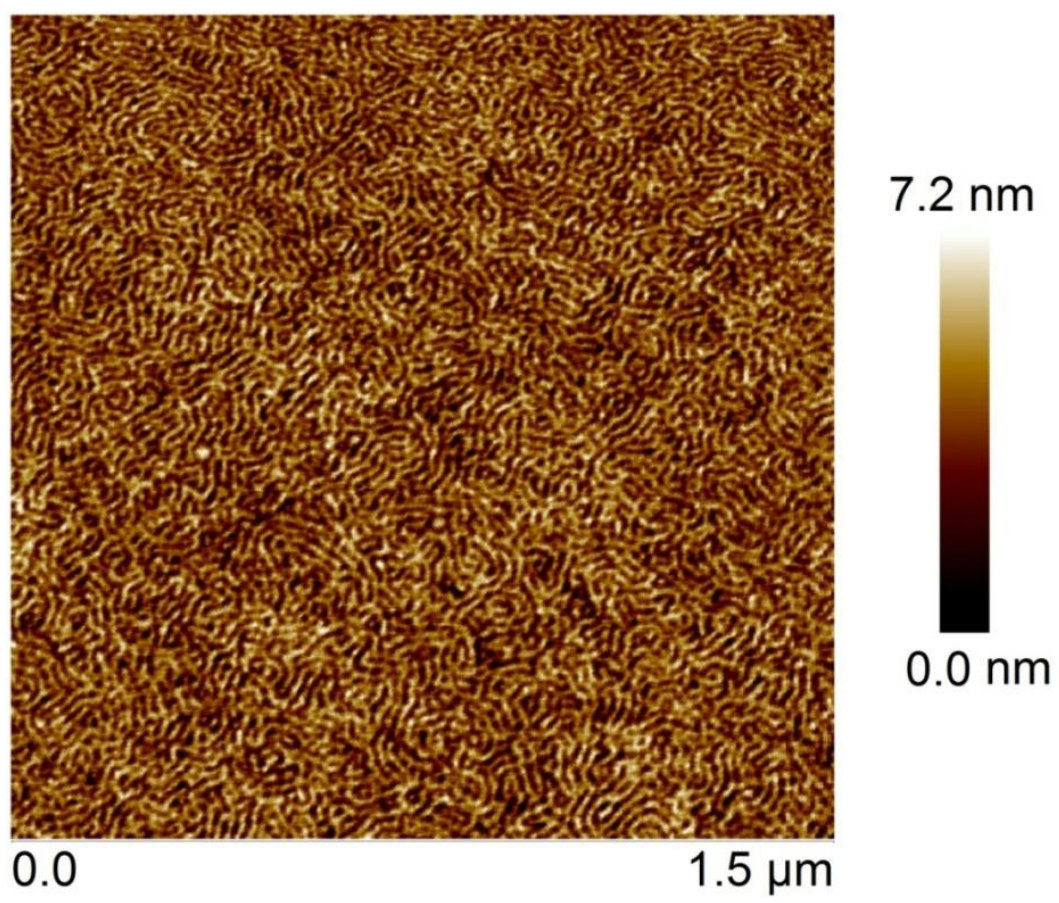

Abbildung 4-61 AFM-Höhenprofil von $\mathrm{P}\left(\mathrm{MMA}_{48}-c o-\mathrm{CMA}_{4}\right)-b$ - $\mathrm{PBA}_{124}$ (Probe $\mathbf{X}$ ) mit $F_{\mathrm{BA}}=69$ mol\%. Die Probe wurde bei $160^{\circ} \mathrm{C}$ über Nacht getempert. Cantilever: FESPA. PF setpoint automatisch gesetzt. Auflösung jew. 512 x 512 Pixel.

Das Phasenverhalten des BCPs $\mathbf{X}$ bei erhöhten Temperaturen konnte mittels in-situ-AFM-Messungen nicht vorgenommen werden, da schon bei Temperaturen von $T<100^{\circ} \mathrm{C}$ eine hohe Klebrigkeit des Polymers festgestellt wurde. Aufgrund zu 
hoher Adhäsion konnte sich der Cantilever nicht von der Probenoberfläche zurückziehen. Dementsprechend wurde der ungeordnete Zustand des Polymersystems mittels eines ex-situ-Verfahrens nachgewiesen. Dafür wurde eine 2,5 Gew.\%-ige Polymerlösung des BCPs $\mathbf{X}$ per spincoating auf ein vorbehandeltes Glassubstrat aufgetragen und im Anschluss bei $160^{\circ} \mathrm{C}$ über Nacht getempert und mittels AFM analysiert, wobei die oben beschriebene Phasenseparation von Probe $\mathbf{X}$ festgestellt wurde [siehe Abbildung 4-62 a1) und b1)]. Danach wurde der auf das Substrat aufgetragene Polymerfilm in einem Heizblock für $2 \mathrm{~h}$ auf $180^{\circ} \mathrm{C}$ erwärmt, wodurch das Polymer Zeit bekommt eine homogene Phase zu bilden. Im Anschluss wurde das im Gleichgewichtszustand befindliche Polymersystem bei einer Temperatur von $180^{\circ} \mathrm{C}$ für $4 \mathrm{~h}$ mit UV-Licht $(\lambda>310 \mathrm{~nm})$ bestrahlt. Nach der Bestrahlung wurde das Material auf $21^{\circ} \mathrm{C}$ (RT) abgekühlt und mittels AFM untersucht. Die Abbildung 4-62 a1) und a2) zeigen das AFM-Höhenprofil des BCPs $\mathbf{X}$ vor und nach der Bestrahlung mit UV-Licht, wohingegen die Abbildung 4-62 b1) und b2) eine Referenzprobe ohne UV-Bestrahlung darstellt. Die getemperten Ausgangsproben in den Abbildung 4-62 a1) und b1) veranschaulichen den phasenseparierten Zustand des BCPs $\mathbf{X}$, der bei einer Temperatur von ca. $21^{\circ} \mathrm{C}$ den globalen Gleichgewichtszustand des Polymers darstellt. Nach UV-Bestrahlung bei $180^{\circ} \mathrm{C}$ und Abkühlen des Materials auf $21^{\circ} \mathrm{C}$ liegt das System in einem ungeordneten, homogenen Zustand vor und das Höhenprofil zeigt eine signifikante Höhenabnahme [Abbildung 4-62 a2)]. Im Vergleich dazu zeigt die nicht vernetzte Probe in Abbildung 4-62 b2) die erwartete Gleichgewichtsmorphologie bei $21^{\circ} \mathrm{C}$, wobei die Phasenseparation von der Ausgangsprobe in Abbildung 4-62 a1) abweicht und eine Höhenabnahme des Materials erkennbar ist. Das könnte durch den schneller ablaufenden Abkühlvorgang beim Heizblock zustande kommen, wodurch das System nicht genügend Zeit hat, sich vollständig neu anzuordnen. 

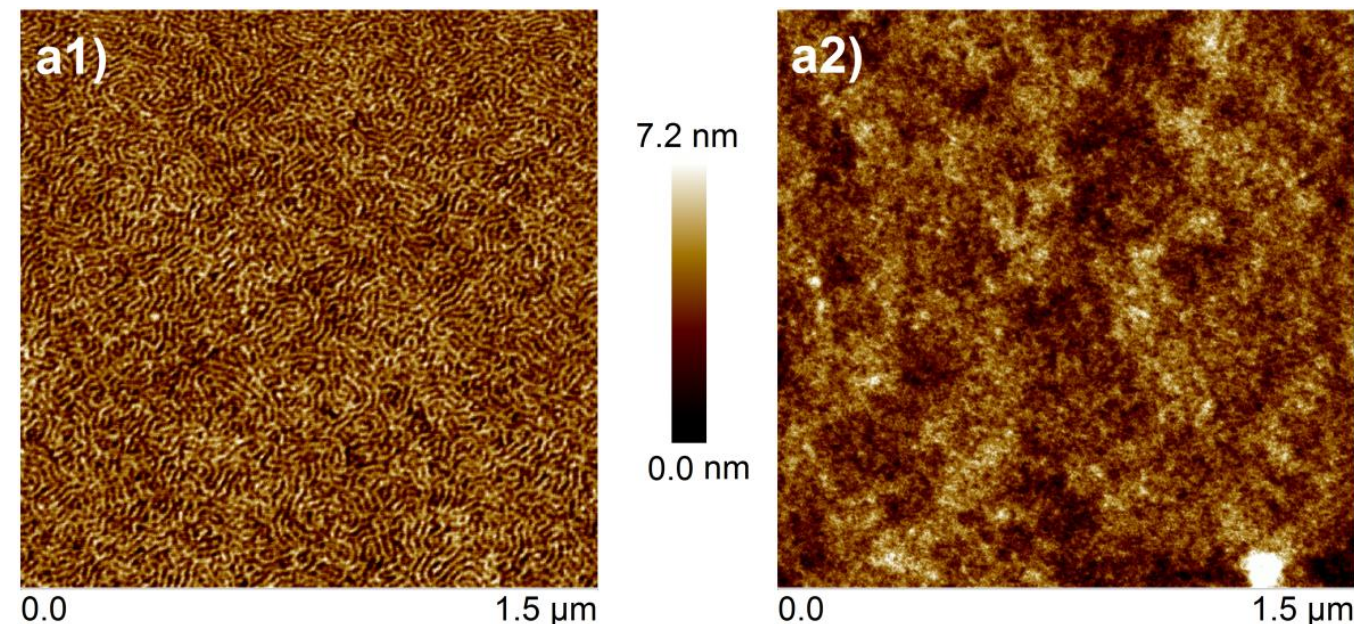

$2.7 \mathrm{~nm}$

0.0

$1.5 \mu \mathrm{m}$

$1.5 \mu \mathrm{m}$
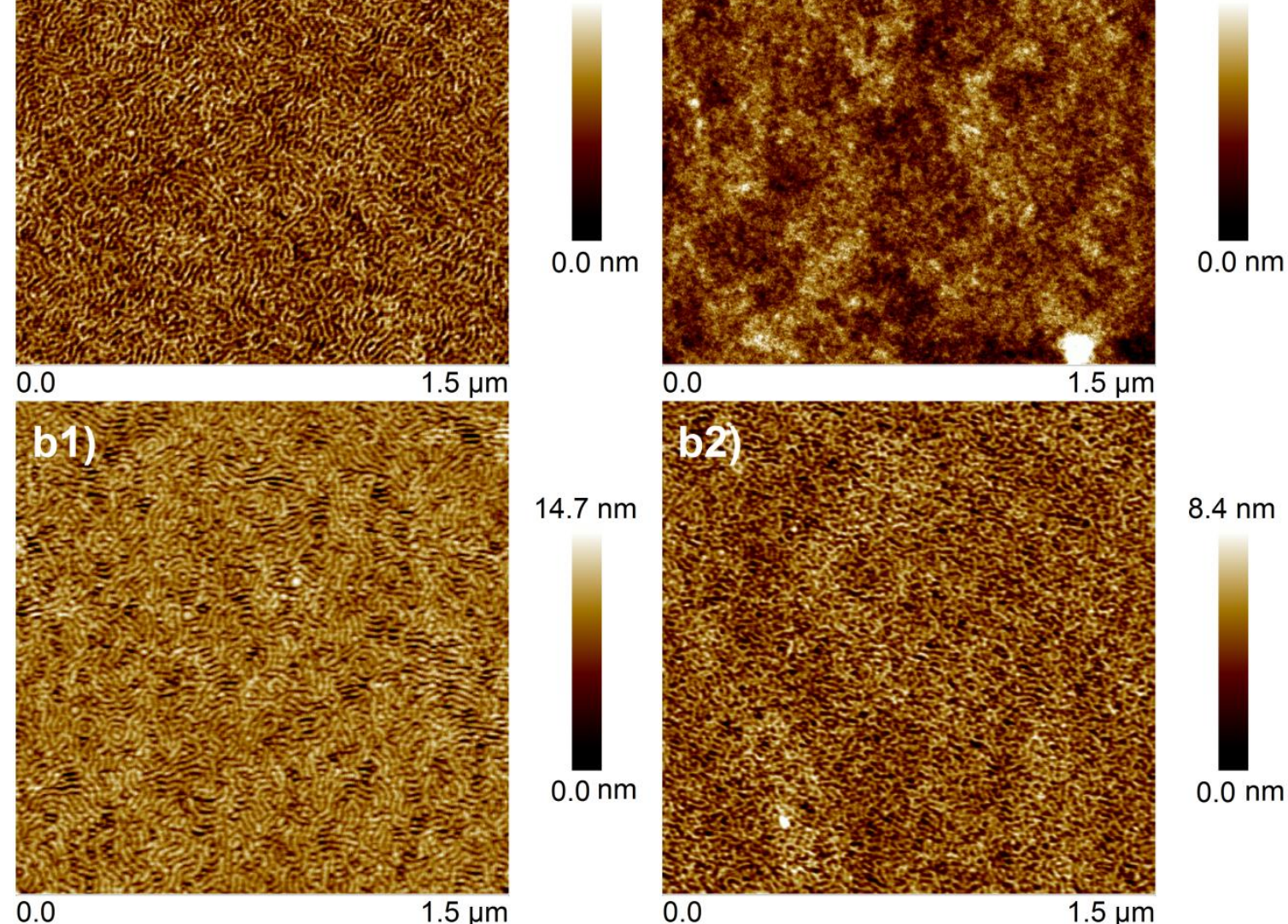

$8.4 \mathrm{~nm}$

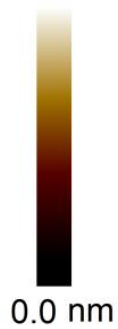

Abbildung 4-62 AFM-Höhenprofile des BCPs $\mathbf{X}$ mit $F_{\mathrm{BA}}=70 \mathrm{~mol} \%$ : a1) Ausgangsprobe bei $160{ }^{\circ} \mathrm{C}$ über Nacht getempert; a2) getemperte Probe nach $4 \mathrm{~h}$ Bestrahlung $(\lambda>310 \mathrm{~nm})$ bei $T=180{ }^{\circ} \mathrm{C}$; b1) Ausgangsprobe bei $160{ }^{\circ} \mathrm{C}$ über Nacht getempert; b2) getemperte Probe, $4 \mathrm{~h}$ auf $180{ }^{\circ} \mathrm{C}$ geheizt und nicht bestrahlt, auf $21^{\circ} \mathrm{C}$ abgekühlt. Alle Proben wurden jew. bei $21^{\circ} \mathrm{C}$ mit dem AFM untersucht. Cantilever: FESPA. Auflösung jew. 512 x 512 Pixel.

Das Höhenprofil des ungeordneten Zustands der Probe $\mathbf{X}$ aus Abbildung 4-62 a2) wird zusätzlich mit dem Profil eines makroRAFT-Agens verglichen, um eine Aussage über die Oberflächenstruktur zu geben. Da das makroRAFT-Agens keine Phasenseparation ausbilden kann, ist in Abbildung 4-63 a) eine glatte, ungeordnete Oberfläche zu erkennen. Im Vergleich dazu zeigt das BCP X in Abbildung 4-63 b) eine identische Probenoberfläche und ein Anzeichen einer MPS ist nicht zu erkennen, wodurch von einem ungeordneten Zustand der Blöcke des vernetzten Polymers $\mathbf{X}$ aus Abbildung 4-62 a2) ausgegangen werden kann. 

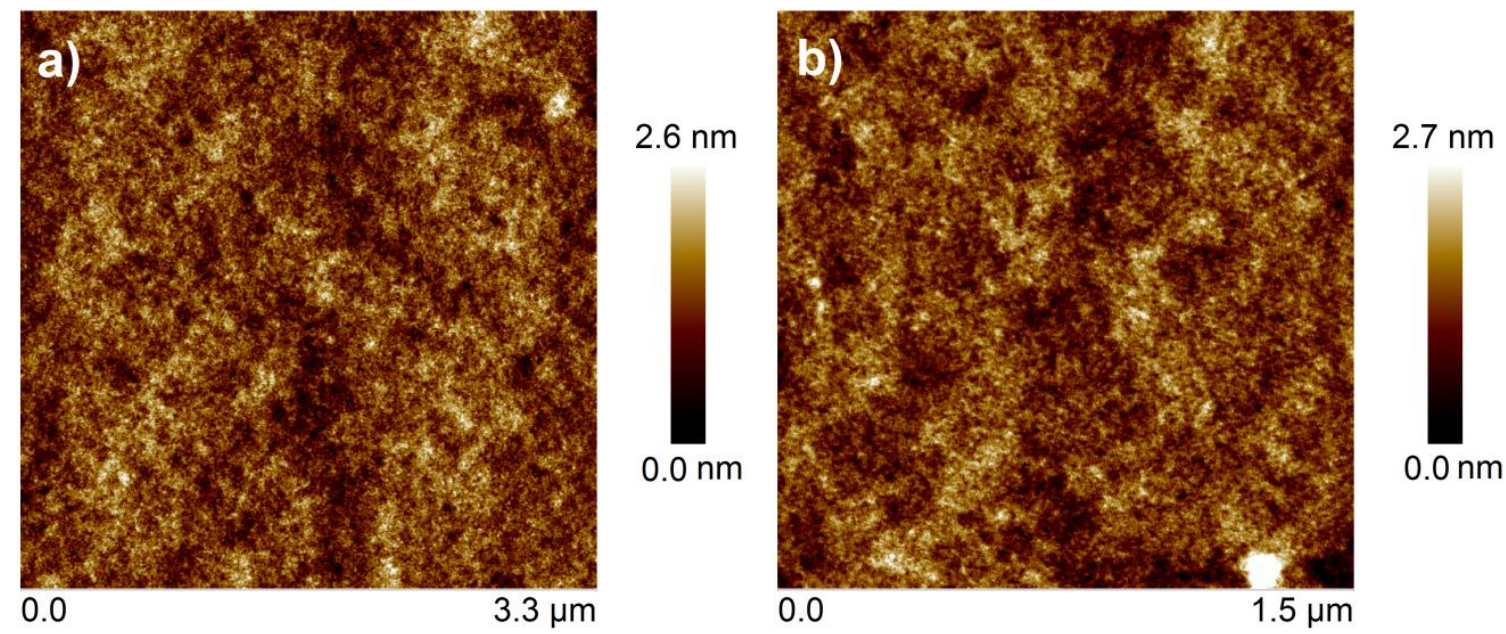

Abbildung 4-63 a) AFM-Höhenprofil eines makroRAFT-Agens P(MMA-co-CMA) nach dem Tempern bei $160^{\circ} \mathrm{C}$ über Nacht; b) AFM-Höhenprofil des BCPs $\mathbf{X}$ nach dem Tempern und $4 \mathrm{~h}$ Bestrahlung $(\lambda>310 \mathrm{~nm})$ bei $T=180^{\circ} \mathrm{C}$ [siehe Abbildung 4-62 a2)]. Beide Proben wurden jew. bei $21{ }^{\circ} \mathrm{C}$ mit dem AFM untersucht. Cantilever: FESPA. Auflösung jew. 512 x 512 Pixel.

Der bei hohen Temperaturen auftretende ungeordnete Zustand des Blockcopolymersystems $\mathbf{X}$ wurde durch die Vernetzungsreaktion der CoumarinEinheiten erfolgreich eingefroren [Abbildung 4-62 a2)], wodurch das System beim Abkühlen daran gehindert wird, in die phasenseparierte Struktur überzugehen. Folglich befindet sich das Polymersystem in Abbildung 4-62 a2) in einem Nichtgleichgewichtszustand. Im Vergleich zum BCP P(MMA48-co-CMA 4$)-b-\mathrm{PBA}_{124}$ $\left(\mathrm{X}, \bar{M}_{\mathrm{n}}=22200 \mathrm{~g} / \mathrm{mol}, F_{\mathrm{BA}}=69 \mathrm{~mol} \%\right)$ zeigt das Polymer P(MMA95-Co-CMA9)- $b$ $\mathrm{PBA}_{229}$ (VII, $\bar{M}_{\mathrm{n}}=42100 \mathrm{~g} / \mathrm{mol}, \quad F_{\mathrm{BA}}=68 \mathrm{~mol} \%$ ) bei AFM-Untersuchungen bei Temperaturen bis zu $210{ }^{\circ} \mathrm{C}$ kein Auftreten des Ordnungs-Unordnungs-Übergangs, wodurch gezeigt werden kann, dass sich das System durch die Verringerung des Polymerisationsgrades $N$ des BCPs mehr im weak segregation limit (WSL, siehe Abschnitt 2.5.1) befindet. In diesem Bereich ist die Unverträglichkeit der Blöcke gering, wodurch das Material einen niedrigeren und somit experimentell ermittelbaren ODT besitzt. 


\section{Fazit und Ausblick}

Das Ziel der vorliegenden Arbeit war es, phasenseparierende Diblockcopolymere mit eingebauten photo-vernetzbaren Einheiten zu synthetisieren und hinsichtlich ihrer Topographie, mechanischen Eigenschaften sowie ihres Phasenverhaltens in Abhängigkeit von der Temperatur zu analysieren. Weiterhin wurde der Einfluss der Vernetzungen auf die Selbstorganisation in Blockcopolymeren untersucht. Dazu wurden Blockcopolymersysteme bestehend aus Methacrylaten und Acrylaten mittels RAFT-Polymerisation synthetisiert, wobei zusätzlich photo-vernetzbare Einheiten in eine Blockkomponente des Polymers eingebaut wurden, um eine reversible Vernetzung des Blockcopolymersystems durch UV-Bestrahlung zu ermöglichen.

Zunächst wurde der Einbau der vernetzungsfähigen Gruppen in Form von Coumarin-Derivaten mittels RAFT-Polymerisation mit Methylmethacrylat (MMA) und $n$-Butylacrylat (BA) untersucht. Dabei wurde festgestellt, dass die Copolymerisation von 7-(2-Methacryloyloxyethoxy)-4-methylcoumarin (CMA) mit MMA mittels RAFT-Polymerisation erfolgreich durchgeführt werden kann, wobei Copolymere mit einer engen Molmassenverteilung erhalten wurden. Die Reaktion findet in einem angemessenen Zeitintervall statt und kann somit als geeigneter Weg für den Einbau photo-vernetzbarer Einheiten in das Polymer genutzt werden. Der erfolgreiche Einbau des CMA-Monomers in das Copolymer und die Zusammensetzung der statistischen Copolymere wurde mittels NMRSpektroskopie bestimmt, wobei in zukünftigen Arbeiten zusätzlich die Copolymerisationsparameter für das oben beschriebene Copolymer-System bestimmt werden können.

In einem zweiten Schritt wurden die synthetisierten Copolymere als makroRAFTAgenzien für eine Blockcopolymerisation verwendet, wobei der zweite Block im Vergleich zum ersten gegensätzliche Eigenschaften besitzt, um die Mikrophasenseparation des Polymersystems zu begünstigen. Da das makroRAFT-Agens $\mathrm{P}(\mathrm{MMA}-\mathrm{co}-\mathrm{CMA})$ bei Raumtemperatur ein hartes Material ist, wurde für den zweiten Block das Monomer BA gewählt, da dieses aufgrund der niedrigen Glasübergangstemperatur ein sehr weiches Material bildet. Zusätzlich zu dem Blockcopolymer P(MMA-co-CMA)- $b$-PBA wurde ein weiteres Polymersystem synthetisiert, in dem beide Blockkomponenten aus Methacrylat-Monomeren 
aufgebaut werden (P(MMA-co-CMA)- $b$-PBMA). Aufgrund der Ähnlichkeit der Monomereinheiten und der damit einhergehenden niedrigeren Entmischungstendenz der Blockcopolymere im Vergleich zum ersten System wurde die Mikrophasenseparation beider Blockcopolymere in Abhängigkeit von der Temperatur untersucht. Die Zusammensetzungen der Blockcopolymersysteme wurden wie in dem Fall des makroRAFT-Agens mittels NMR-Spektroskopie bestimmt. Insgesamt wurden erfolgreiche Blockcopolymerisationen für beide Systeme festgestellt, wobei für das Polymer P(MMA-co-CMA)- $b$-PBA mit größeren Ausgangsblöcken $\left(\bar{M}_{\mathrm{n}}>47000 \mathrm{~g} / \mathrm{mol}\right)$ ein weniger kontrolliertes Polymerisationsverhalten ermittelt wurde. In diesem Fall zeigt das makroRAFT-Agens eine niedrigere effektive Konzentration der RAFT-Endgruppen, wobei diese Konzentration entscheidend ist für das weitere Kettenwachstum. Dadurch rücken Nebenreaktionen wie z. B. Verzweigung und Terminierung mehr in den Vordergrund und die Kontrolle über die Polymerisation wird minimiert. Insgesamt wurde eine große Vielfalt von Blockcopolymeren synthetisiert, die zum einen in ihren einzelnen Blocklängen und somit in der Zusammensetzung des Polymers variieren und zum anderen in der Molmasse des Blockcopolymers.

Es wurden umfangreiche Untersuchungen an den Blockcopolymersystemen P(MMA-co-CMA)- $b$-PBA und P(MMA-co-CMA)- $b$-PBMA durchgeführt. So wurden die thermischen Eigenschaften der Blockcopolymere mit Hilfe der dynamischmechanischen Analyse (DMA) untersucht. Dabei konnten für beide Systeme jeweils zwei Glasüberganstemperaturen $\left(T_{\mathrm{g}}\right)$ bestimmt werden, was ein eindeutiges Indiz für Mikrophasenseparation ist. Außerdem wurde bei dem BCP P(MMA-co-CMA)- $b$ PBA mit einer geringen Molmasse $\left(\bar{M}_{\mathrm{n}}=22200 \mathrm{~g} / \mathrm{mol}\right)$ ein Hinweis auf die Existenz des Ordnungs-Unordnungs-Übergangs festgestellt.

Weiterhin wurde die Mikrophasenseparation der unterschiedlichen Polymersysteme mittels AFM umfangreich untersucht. Dabei wiesen die Blockcopolymere eine vielfältige Morphologie (Sphären, Zylinder, perforierte Lamellen) in Abhängigkeit von ihrer jeweiligen Zusammensetzung auf. Die Domänenabstände für die P(MMA-co-CMA)-Blöcke der Blockcopolymere wurde mittels PSD-Funktion bestimmt, wobei für die identischen makroRAFT-Agenzien, die in unterschiedlichen BCP eingebaut wurden, ähnliche Werte für die Domänenabstände ermittelt wurden. Bei beiden Systemen wurde das Phasenverhalten bei erhöhten Temperaturen mittels in-situ-AFM-Untersuchungen analysiert. Dabei lagen beide Blockcopolymersysteme in einem Temperaturbereich von $21^{\circ} \mathrm{C}$ bis $190{ }^{\circ} \mathrm{C}$ bzw. $210^{\circ} \mathrm{C}$ in einem konstant phasenseparierten Zustand vor. Lediglich bei dem BCP 
P(MMA-co-CMA)- $b$-PBA wurde eine Abnahme der Domänenabstände für die Zylinder bei einer Temperatur von ca. $180{ }^{\circ} \mathrm{C}$ festgestellt, wobei das vollständige Auflösen der Zylinderstruktur ausblieb. Folglich wurde für beide Blockcopolymersysteme kein Ordnungs-Unordnungs-Übergang (ODT) bei experimentell zugänglichen Versuchsbedingungen mittels in-situ-AFM-Untersuchungen ermittelt, da bei langkettigen Blockcopolymeren der ODT oft weit über der thermischen Stabilitätsgrenze liegt. Allerdings konnte bei dem BCP P(MMA-coCMA)- $b$-PBA durch die Verringerung des Polymerisationsgrades $N$ ein niedrigerer ODT festgestellt und mittels AFM-Messungen nachgewiesen werden.

Der für die Ausbildung der Mikrophasenseparation entscheidende Tempervorgang der Blockcopolymere wurde für das System P(MMA-co-CMA)- $b$-PBA detailliert untersucht. Dabei wurde eine Abhängigkeit des Prozesses von der Temperzeit und -temperatur festgestellt, wobei lediglich die Ausbildung der Gleichgewichtsmorphologie von den Temperbedingungen abhängig ist und nicht der Domänenabstand der P(MMA-co-CMA)-Blöcke.

Die mechanischen Eigenschaften des BCPs P(MMA-co-CMA)- $b$-PBA wurden mittels AFM und DMA untersucht. Dabei wurde eine gute Übereinstimmung der lokalen mit den globalen DMT-Moduln ermittelt, wobei insgesamt eine kleinere Größenordnung für die Moduln als für reines PMMA festgestellt wurde, was auf den Einfluss des eingebauten weichen PBA-Blocks zurückzuführen ist.

Die reversible photochemische Vernetzungsreaktion wurde an dünnen Polymerfilmen bei unterschiedlichen Temperaturen und Bestrahlungszeiten untersucht. Der Erfolg des reversiblen Vernetzungsprozesses wurde mittels UV/Vis-Spektroskopie und AFM-Untersuchungen nachgewiesen. Dabei wurde festgestellt, dass bereits 7 mol\% vernetzbare CMA-Einheiten in dem Ausgangsblock zu einer erfolgreichen Vernetzung des BCPs führen, wobei in zukünftigen Arbeiten die Variation der Vernetzungsdichte noch untersucht werden sollte. Der Einfluss der Vernetzungen auf die mechanischen Eigenschaften des Polymersystems wurde mittels DMA-Untersuchungen nachgewiesen, wobei eine Zunahme des Moduls mit zunehmender Bestrahlungszeit $(\lambda>310 \mathrm{~nm})$ festgestellt wurde. Zusätzlich sollte in der Zukunft der Prozess der Dimerspaltung mittels DMAMessungen genauer analysiert werden. Die Effizienz der Vernetzungsreaktion wird besonders anhand der AFM-Untersuchungen deutlich, wobei gezeigt werden konnte, dass die Selbstorganisation in den BCP durch die topologischen Einschränkungen durch Vernetzung des Systems verhindert wird. Dafür wurde das 
BCP $\mathrm{P}(\mathrm{MMA}-\mathrm{co}-\mathrm{CMA})-b$-PBA in seinem ungeordneten, homogenen Zustand bei hohen Temperaturen vernetzt und mittels AFM-Untersuchungen wurde nach dem Abkühlen keine phasenseparierte Struktur festgestellt, wodurch gezeigt werden konnte, dass sich das BCP in einem Nichtgleichgewichtszustand befindet. Die Untersuchung der Relaxation des Systems durch die Dimerspaltung im Nichtgleichgewichtszustand stellt eine interessante Aufgabe für zukünftige Arbeiten dar.

Weitere Untersuchungen des Polymersystems könnten zur vollständigen Aufklärung beitragen. So konnten zwar durch die AFM-Untersuchungen detaillierte Informationen zur Oberfläche des Materials ermittelt werden, allerdings wären zusätzliche 3D-Untersuchungen der geordneten Phasen z. B. durch Röntgentomographie oder STED-Mikroskopie wünschenswert. Weiterhin sind Untersuchungen der Morphologie und Materialeigenschaften der Blockcopolymere während mechanischer Beanspruchung denkbar. Diese Strukturanalyse könnte insitu durch ein eingebautes Zugmodul im AFM durchgeführt werden oder ex-situ mittels Zugversuchen erfolgen. 


\section{Experimentalteil}

\subsection{Chemikalien}

Sofern nicht anders angegeben ist, wurden alle kommerziell erhältlichen Chemikalien (von ABCR, Acros Organics, Merck, Roth, VWR und Sigma Aldrich) mit einer Reinheit von mindestens $95 \%$ ohne weitere Aufreinigung verwendet. Für Synthesen wurden Lösungsmittel in pro analysi-Qualität eingesetzt.

\subsection{Monomere}

Methylmethacrylat (Sigma Aldrich, 99\%, stabilisiert mit Hydrochinonmonomethylether), $n$-Butylacrylat (Sigma Aldrich, $\geq 99 \%$, stabilisiert mit Hydrochinonmonomethylether), Butylmethacrylat (Sigma Aldrich, $99 \%$, stabilisiert mit Hydrochinonmonomethylether) und 2-Hydroxyethylmethacrylat (Sigma Aldrich, 97 \%, stabilisiert mit Hydrochinonmonomethylether) wurden vor der Verwendung durch Inhibitor Remover (Sigma Aldrich) filtriert, um den Inhibitor abzutrennen. Alle Monomere wurden bei $-18^{\circ} \mathrm{C}$ gelagert.

\subsection{Initiator}

2,2'-Azobis-(iso-butyronitril) (AkzoNobel, 98\%) wurde aus Methanol umkristallisiert, im Vakuum getrocknet und bei $-18^{\circ} \mathrm{C}$ gelagert.

\subsection{RAFT-Agenz}

4-Cyano-4-[(dodecylsulfanylthiocarbonyl)sulfanyl]-pentansäure (Sigma Aldrich, $97 \%$ ) wurde als RAFT-Agenz verwendet, da es sich besonders gut für die Polymerisation von Methacrylaten und Acrylaten eignet. ${ }^{180,181}$ Das RAFT-Agenz ist in Schema 6-1 dargestellt. 


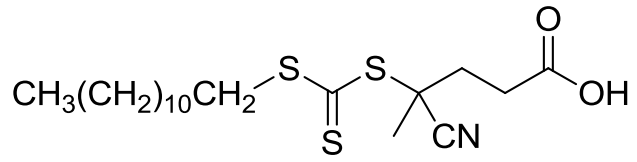

Schema 6-1 Chemische Struktur von 4-Cyano-4-[(dodecylsulfanylthiocarbonyl)sulfanyl]pentansäure.

\subsection{Spektroskopische und analytische Methoden}

\section{Gelpermeationschromatographie (GPC)}

Die Molmassenverteilungen der Methylmethacrylat-, Butylacrylat- und Hydroxylethylmethacrylatpolymere, sowie der Blockcopolymere, Copolymere und der Homopolymere des UV-Monomers 7-(2-Methacryloyloxyethoxy)4-methylcoumarin (CMA) wurden mit einem SEC Analysis System 1260 Infinity von PSS Agilent gemessen. Das System besteht aus einer PSS Agilent Technologies 1260 Iso Pump G1310B (HPLC-Pumpe), einem Agilent 1260 ALS G1329B Autosampler, einem Agilent 1260 ALS Injektor, einer Vorsäule (PSS SDV, 8x50 mm, Partikelgröße $5 \mu \mathrm{m}$ ), drei Trennsäulen (PSS SDV, 8 x 300 mm, Partikelgröße: $5 \mu \mathrm{m}$, Porengröße $10^{5} \AA, 10^{3} \AA$ und $10^{2} \AA$ ) und den Detektoren. In dieser Arbeit wurden der UV-Detektor PSS Agilent Tecchnologies 1260 VWDVL bei einer Wellenlänge von 310 nm, sowie der RI-Detektor PSS Agilent Technologies 1260 RID benutzt. Als Laufmittel diente THF (HPLC-grad) mit Toluol ( $>99,7 \%$, trocken) als interner Standard (Fließgeschwindigkeit $1,0 \mathrm{~mL} / \mathrm{min}$ bei $35^{\circ} \mathrm{C}$ ). Das System wurde mit Polystyrol-Standards mit niedriger Dispersität von PSS kalibriert. Die Kalibrierung für Polymethylmethacrylat und Polybutylacrylat war über die tabellierten MarkHouwink-Parameter (für MMA: $\mathrm{K}=0,01298 \mathrm{~cm}^{3} / \mathrm{g}, \mathrm{a}=0,688$ und für $\mathrm{BA}$ : $\mathrm{K}=0,0122 \mathrm{~cm}^{2} / \mathrm{g}$, a = 0,700)182,183 möglich. Zur Auswertung wurde die Software PSS WinGPC verwendet. Die detektierten Intensitäten wurden auf 1 normiert.

\section{Kernspinresonanzspektroskopie (NMR)}

${ }^{1} \mathrm{H}-\mathrm{NMR}$ wurden mit dem Modell Unity 300 (300 MHz) von Varian oder mit dem Modell Avance III (300 MHz) von Bruker bei Raumtemperatur gemessen. Als Lösungsmittel wurden $\mathrm{CDCl}_{3}-\mathrm{d}_{1}$ und DMSO- $\mathrm{d}_{6}$ von Deutero verwendet. Für die Auswertung wurde das Programm MestReNova 7.1 verwendet. Die Multiplizitäten wurden folgendermaßen abgekürzt: s (Singulett), $d$ (Dublett), $t$ (Triplett), $q$ (Quartett), quin (Quintett), dd (Dublett von Dublett), m (Multiplett). 


\section{Massenspektrometrie}

Die ESI-Spektren wurden auf einem Bruker Daltonik ESI-ToF-MS (MICRO-TOF) in den Laboren der Zentralen Analytik des Institutes für Organische Chemie an der Georg-August-Universität Göttingen gemessen. Alle Messungen wurden bei einer Kapillartemperatur von $180^{\circ} \mathrm{C}$ und einer Spannung von $4,5 \mathrm{kV}$ detektiert.

\section{Rasterkraftmikroskop (AFM)}

Die AFM-Messungen wurden mit einem Multimode 8 AFM ausgestattet mit einem NanoScope V Kontroller von der Firma Veeco/Digital Instruments (Santa Babara, CA) durchgeführt. Für die Messungen wurde der Modus PeakForce-Quantitative Nanomechnaical Mapping ${ }^{\mathrm{TM}}$ (PeakForce-QNM ${ }^{\mathrm{TM}}$ ) verwendet. Als Cantilever dienten der FESPA (Material: Si, Resonanzfrequenz $\sim 75 \mathrm{kHz}$, Federkonstante $=2,8 \mathrm{~N} / \mathrm{m}$, Spitzenradius $=8 \mathrm{~nm}$, Bruker AFM Probes), der RFESP (Material: Si , Resonanzfrequenz $\sim 75 \mathrm{kHz}$, Federkonstante $=3 \mathrm{~N} / \mathrm{m}$, Spitzenradius $=8 \mathrm{~nm}$, Bruker AFM Probes) und der ScanAsystAir (Material: SiN, Resonanzfrequenz $\sim 70 \mathrm{kHz}$, Federkonstante $=0,4 \mathrm{~N} / \mathrm{m}$, Spitzenradius $=2 \mathrm{~nm}$, Bruker AFM Probes). Alle Messungen wurden unter Argon-Atmosphäre durchgeführt. Die Scanrate betrug 0,5 bis $1 \mathrm{~Hz}$. Zur Datenauswertung wurde die NanoScope Analysis Version 1.40 verwendet.

\section{UV/Vis-Spektroskopie}

UV/Vis-Spektren wurden mit einem Cary 300-Spektrometer von Agilent in einem Wellenlängenbereich von 250 bis $600 \mathrm{~nm}$ aufgenommen.

\section{UV-Lampen}

Für die Bestrahlungsversuche wurden zwei unterschiedliche UV-Lampen benutzt. Für die UV-Vis-Messungen wurde die Bestrahlungskammer UVACUBE inert von der Firma Hönle verwendet. Diese wurde mit einer Quecksilberlampe (1500 W) und einem Lichtleiter (Serie 250) von Lumatec ausgestattet. Für die DMA- und AFM-Proben wurde die UV-Lampe XH 100 von der Firma Müller verwendet. Diese wurde mit einer Quecksilberlampe $(100 \mathrm{~W})$ und dem Lichtleiter LLEF von Müller bestückt.

Alle untersuchten Proben wurden mit UV-Licht unterschiedlicher Wellenlänge bestrahlt. Dafür wurde der Langpassfilter N-WG305 SCHOTT (ITOS) mit einer Wellenlänge von $\lambda>310 \mathrm{~nm}$ verwendet sowie der Bandpassfilter F39-HG1 (AHF) mit einer Wellenlänge $\lambda=254 \mathrm{~nm}$. 


\section{Thermogravimetrische Analyse (TGA)}

Die thermogravimetrischen Untersuchungen wurden mit dem Gerät STA 449 C Jupiter der Firma Netzsch durchgeführt. Ein Aluminiumtiegel mit $20 \mathrm{mg}$ Polymer wurde auf $500{ }^{\circ} \mathrm{C}$ unter Argonatmosphäre erhitzt. Die Heizrate betrug $5 \mathrm{~K} / \mathrm{min}$.

\section{Dynamisch-mechanische Analyse (DMA)}

Die dynamisch mechanischen Messungen wurden mit dem Gerät DMA 8000 der Firma Perkin Elmer durchgeführt. Bei diesem Gerät wurden zwei unterschiedliche Messgeometrien verwendet, die in Abschnitt 6.6.5 näher erläutert werden. Die Steuerung der Messungen erfolgt über die mitgelieferte Software DMA Control der Firma Perkin Elmer. Besonderer Dank für die durchgeführten DMA-Messungen gilt Stefanie Finkhäuser aus dem Institut für Physik der Universität Göttingen.

\section{Ellipsometrie}

Die ellipsometrischen Untersuchungen wurden mit einem Nanofilm EP4 Ellipsometer der Firma Accurion durchgeführt. Bei diesem Gerät handelt es sich um ein Imaging Ellipsometer, das als Messverfahren die Null-Ellipsometrie verwendet. Gemessen wurde mit einer laser stabilized xenon arc lamp mit einem Wellenlängenbereich von 360 bis $800 \mathrm{~nm}$. Außerdem verfügt das Ellipsometer über einen internal solid state Laser, der eine Wellenlänge von $658 \mathrm{~nm}$ besitzt. Für Bildaufnahmen ist eine CCD Kamera mit 1392 x 1040 Pixeln und ein Objektiv mit 10-facher Vergrößerung vorhanden. Für die Auswertung der Messdaten steht da Programm EP4-Model zur Verfügung. Für einige Materialien sind vorgefertigt Modelle mit den wichtigen Materialeigenschaften wie Brechungsindex und Reflexionskoeffizient vorhanden. Die Werte der analysierten Proben wurden mit der Methode Cauchy angepasst. 


\subsection{Durchführung der Experimente}

\subsubsection{Synthese von 7-(2-Methacryloyloxyethoxy)-4- methylcoumarin (CMA) und 7-Acryloyloxyethoxy-4- methylcoumarin (CA)}
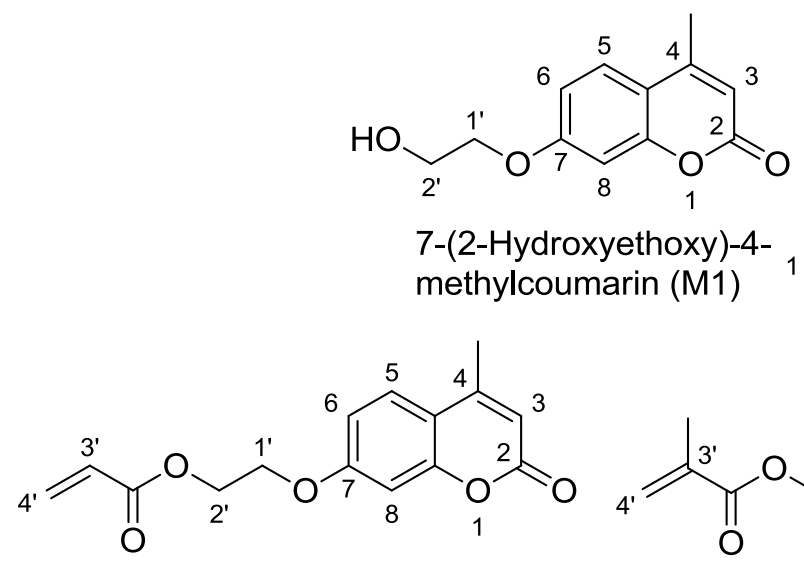

7-Acryloyloxyethoxy4-methylcoumarin<smiles>C=C(C)C(=O)OCCOc1ccc2c(C)cc(=O)oc2c1</smiles>
(CA)

7-(2-Methacryloyloxyethoxy)4-methylcoumarin (CMA)

Für die Synthese der UV-aktiven Comonomere 7-Acryloyloxyethoxy4-methylcoumarin (CA) und 7-(2-Methacryloyloxyethoxy)-4-methylcoumarin (CMA) wurde zuerst die Vorstufe 7-(2-Hydroxyethoxy)-4-methylcoumarin (M1) mit Hilfe der Synthesemethode nach Zhang et al. hergestellt. ${ }^{184}$ Dafür wurden 7-Hydroxy-4-methylcoumarin (4,40 g, 25 mmol, 1 Äq.), Kaliumcarbonat (6,93 g, $50 \mathrm{mmol}, 2$ Äq.), Kaliumiodid $(0,253 \mathrm{~g}, 6 \mathrm{~mol} \%)$ und 2-Chlorethanol (4,03 g, $50 \mathrm{mmol}, 3,4 \mathrm{~mL}, 2$ Äq.) in DMF (50 mL) gelöst. Diese Mischung wurde auf $110{ }^{\circ} \mathrm{C}$ erhitzt und über Nacht gerührt. Nach Abkühlen der Reaktionsmischung auf Raumtemperatur wurde sie mit Essigsäureethylether extrahiert. Die organische Phase wurde über Magnesiumsulfat getrocknet. Nach Abdampfen des Lösungsmittels im Vakuum wurde das Produkt als weißer Feststoff erhalten (4,96 g, 22,5 mmol, $90 \%)$.

1H-NMR (300 MHz, DMSO) $\delta(\mathrm{ppm}): \quad 2,40$ (s, $\left.3 \mathrm{H},-\mathrm{C}^{4}-\mathrm{CH}_{3}\right), 3,73$ (t, $2 \mathrm{H}, \mathrm{HO}-$ $\left.\mathrm{C}^{1} \underline{\mathrm{H}}_{2}-\mathrm{C}^{2} \mathrm{H}_{2}-\right), \quad 4,10 \quad\left(\mathrm{t}, \quad 2 \mathrm{H}, \quad \mathrm{HO}-\mathrm{C}^{1} \mathrm{H}_{2}-\right.$ $\mathrm{C}^{\prime} \underline{\mathrm{H}}_{2}^{-}$), 4,93 (t, $1 \mathrm{H}, \mathrm{HO}-$ ), 6,21 (s, $1 \mathrm{H},-\mathrm{C}^{3}-$ $\mathrm{H}), 6,96\left(\mathrm{~s}, 1 \mathrm{H},-\mathrm{C}^{7}-\mathrm{H}\right), 6,98\left(\mathrm{~s}, 1 \mathrm{H},-\mathrm{C}^{8}-\mathrm{H}\right)$, 7,70 (d, $\left.1 \mathrm{H},-\mathrm{C}^{6}-\mathrm{H}\right)$ 
ESI-MS:

$221,1[\mathrm{M}+\mathrm{H}]^{+}, \quad 243,1 \quad[\mathrm{M}+\mathrm{Na}]^{+}, \quad 463,2$ $[2 \mathrm{M}+\mathrm{Na}]^{+}$

Das Produkt M1 wurde im nächsten Syntheseschritt ohne weitere Aufreinigung verwendet. CA wurde nach einer abgewandelten Synthesevorschrift von Shaughnessy et al. durchgeführt. ${ }^{185}$ In Acetonitril $(115 \mathrm{~mL})$ wurden unter Argon M1 (2,29 g, $10 \mathrm{mmol}, 1,5$ Äq.) gelöst und mit einem Eisbad auf $0{ }^{\circ} \mathrm{C}$ gekühlt. Im Anschluss wurden zuerst Triethylamin (2,08 g, $20 \mathrm{mmol}, 2,85 \mathrm{~mL}, 2$ Äq.) tropfenweise hinzugegeben und danach Acryloylchlorid (1,36 g, $15 \mathrm{mmol}, 1,22 \mathrm{~mL}$, 1,5 Äq.). Nach Entfernung des Eisbades wurde die Reaktionslösung für 2,5 $\mathrm{h}$ bei Raumtemperatur gerührt. Das entstandene Salz wurde abfiltriert und das Lösungsmittel wurde im Vakuum abgedampft. Im Anschluss wurde der gelbe Feststoff in $45 \mathrm{~mL}$ Dichlormethan gelöst und zweimal mit je $40 \mathrm{~mL}$ wässriger Salzsäure-Lösung und einmal mit $40 \mathrm{~mL}$ Wasser extrahiert. Die organische Phase wurde über Magnesiumsulfat getrocknet und das Lösungsmittel wurde im Vakuum entfernt. Das Rohprodukt wurde aus einer Methanol-Ethanol-Mischung (1:1) bei $40{ }^{\circ} \mathrm{C}$ umkristallisiert. Das Produkt wurde als weißer Feststoff erhalten $(2,55 \mathrm{~g}$, 9,3 mmol, $93 \%$ ).

1H-NMR (300 MHz, DMSO) $\delta(\mathrm{ppm}): \quad 2,40\left(\mathrm{~s}, 3 \mathrm{H},-\mathrm{C}^{4}-\mathrm{CH}_{3}\right), 4,38$ (t, $2 \mathrm{H},-0-\mathrm{C}_{2}-$ $\mathrm{CH}_{2}-$ ), 4,48 (t, $2 \mathrm{H},-0-\mathrm{CH}_{2}-\mathrm{C}_{2}-$ ) , 5,96 (dd, $1 \mathrm{H}, \mathrm{C}^{4} \underline{\mathrm{H}}_{2}=\mathrm{C}^{3} \mathrm{H}-\mathrm{C}-$ ), 6,23 (dd, $1 \mathrm{H},-\mathrm{C}^{3}-\mathrm{H}$ ), 6,35 (m, 2 H, - $\mathrm{C}^{4} \underline{\mathrm{H}} 2-\mathrm{C}^{3} \underline{\mathrm{H}}-\mathrm{C}-$ ) , 7,02 (m, 2 $\mathrm{H}$, , - $\left.\mathrm{C}^{7}-\mathrm{H},-\mathrm{C}^{8}-\mathrm{H}\right), 7,69\left(\mathrm{~d}, 1 \mathrm{H},-\mathrm{C}^{6}-\mathrm{H}\right)$

ESI-MS:

$$
\begin{aligned}
& 297,1[\mathrm{M}+\mathrm{Na}]^{+}, 571,2[2 \mathrm{M}+\mathrm{Na}]^{+}, 845,3 \\
& {[3 \mathrm{M}+\mathrm{Na}]^{+}}
\end{aligned}
$$

Die Vorstufe M1 wurde weiterhin für die Synthese des Monomers CMA verwendet. Dafür wurde die Synthesemethode nach Jiang et al. herangezogen. ${ }^{162}$ In Chloroform (150 mL) wurden M1 (1,28 g, 6 mmol, 1 Äq.) gelöst und mit einem Eisbad auf $0{ }^{\circ} \mathrm{C}$ gekühlt. Danach wurden Triethylamin $(1,21 \mathrm{~g}, 12 \mathrm{mmol}, 1,66 \mathrm{~mL}$, 2 Äq.) zu der Lösung dazu getropft und danach Methacryloylchlorid 0 (0,94 g, 9 mmol, 0,88 mL, 1,5 Äq.). Nachdem das Eisbad entfernt wurde, konnte sich die Reaktionslösung auf Raumtemperatur erwärmen und wurde über Nacht gerührt. Die Lösung wurde zweimal mit gesättigter Natriumchlorid-Lösung (30 mL) und einmal mit Wasser $(15 \mathrm{~mL})$ gewaschen. Die wässrige Phase wurde mit Essigsäureethylester extrahiert und die zwei organischen Phasen wurden vereint und über Magnesiumsulfat getrocknet. Das Lösungsmittel wurde im Vakuum 
entfernt. Das Rohprodukt wurde mit einer Methanol-Ethanol-Mischung (1:1) bei $40{ }^{\circ} \mathrm{C}$ umkristallisiert und das Produkt wurde als weißer Feststoff erhalten $(1,14 \mathrm{~g}$, $3,9 \mathrm{mmol}, 71 \%)$.

${ }^{1}$ H-NMR (300 MHz, DMSO) $\delta$ (ppm):

$1,88\left(\mathrm{~s}, 3 \mathrm{H}, \mathrm{H}_{2} \mathrm{C}^{4}=\mathrm{C}^{3}-\mathrm{C}_{3} \underline{3}\right), 2,40(\mathrm{~s}, 3 \mathrm{H},-$ $\left.\mathrm{C}^{4}-\mathrm{CH}_{3}\right), 4,38\left(\mathrm{t}, 2 \mathrm{H},-\mathrm{O}-\mathrm{C}_{2}-\mathrm{CH}_{2}-\right), 4,48$

$\left(\mathrm{t}, 2 \mathrm{H},-\mathrm{O}-\mathrm{CH}_{2}-\underline{\mathrm{C}}_{2}-\right), 5,70 \quad(\mathrm{t}, 1 \mathrm{H}$, $\left.\mathrm{C}^{4} \underline{\mathrm{H}}_{2}=\mathrm{C}^{3}-\mathrm{CH}_{3}\right), 6,03\left(\mathrm{~s}, 1 \mathrm{H}, \mathrm{C}^{4} \underline{\mathrm{H}}_{2}=\mathrm{C}^{3}-\right.$ $\left.\mathrm{CH}_{3}\right), 6,22\left(\mathrm{~d}, 1 \mathrm{H},-\mathrm{C}^{3}-\mathrm{H}\right), 6,99(\mathrm{~d}, 1 \mathrm{H}$, , $\left.\mathrm{C}^{7}-\mathrm{H}\right), 7,03\left(\mathrm{dd}, 1 \mathrm{H},-\mathrm{C}^{8}-\mathrm{H}\right), 7,70(\mathrm{~d}, 1 \mathrm{H},-$ $\left.\mathrm{C}^{6}-\mathrm{H}\right)$

ESI-MS:

$311,1[\mathrm{M}+\mathrm{Na}]^{+}, 599,2[2 \mathrm{M}+\mathrm{Na}]^{+}, 887,3$ $[3 \mathrm{M}+\mathrm{Na}]^{+}$

\subsubsection{Polymerisationen}

Alle verwendeten Lösungsmittel und flüssigen Monomere wurden in einem Einhals-Entgasungskolben gegeben und durch jeweils drei Einfrier-EntgasungsAuftau-Zyklen (engl.: freeze-pump-thaw cycles) an einer Hochvakuumpumpe (Edwards EXC 120 oder Pfeiffer TMH 071) vom Sauerstoff befreit. Die Entgasungskolben wurden zusammen mit den Schraubdeckelgläsern, dem RAFT-Agenz (makroRAFT-Agenz im Falle der Blockcopolymerisation), dem Initiator und gegebenenfalls dem Comonomer in eine Glove-Box (MBRAUN Labmaster 130) mit einem Sauerstoffgehalt unter 4 ppm überführt. Dort wurden alle Polymerisationskomponenten unter Argonatmosphäre miteinander vereint. Die Polymerisationslösung wurde im Anschluss auf mehrere Schraubdeckelgläser aufgeteilt. Diese wurden dann für ein bestimmtes Zeitintervall in einen auf $70^{\circ} \mathrm{C} \pm 0,1^{\circ} \mathrm{C}$ vorgeheizten Thermoblock (Roth Rotilabo ${ }^{\circledR}$ H250 oder Kleinfeld MBT250-2) mit Schüttler (Edmund Bühler GmbH) gesetzt und zum Abbruch der Polymerisation in einem Eisbad abgekühlt. Die Polymerisationsumsätze wurden gravimetrisch bestimmt. Dazu wurden die Proben ggf. mit THF gelöst und in der zehnfachen Menge Methanol gefällt. Die Copolymere mit CA/CMA sowie das Homopolymer CMA wurden mit Hilfe der Zentrifuge (2-16KC von Sigma) von der Lösung abgetrennt. Das gefällte Polymer wurde im Vakuumtrockenschrank bis zur Gewichtskonstanz getrocknet. 


\subsubsection{Probenpräparation, Messprobleme und Kalibrierung für AFM}

\section{Probenpräparation}

Als Substrate für die Polymerfilme wurden Silizium (111)-Wafer(Firma Plano) und Glasplättchen (Firma VWR) verwendet. Diese wurden vor dem spincoating-Prozess in einer Plasmaanlage (Typ ZEPTO, Firma electronic diener, Plasmsa-SurfaceTechnology) gereinigt.

Für die Probenpräparation wurden zuerst $5 \mu \mathrm{L}$ einer Polymerlösung mit $1 \%$ des statistischen Copolymers PMMA-co-PBA-co-PHEMA auf ein gereinigtes Substrat getropft und im Anschluss wurde die Schicht mittels eines Spincoaters (WS650MZ-23NPP von Laurell) für $1 \mathrm{~min}$ bei $2000 \mathrm{rpm}$ gleichmäßig verteilt. Die Probe wurde im Vakuumofen bei $160{ }^{\circ} \mathrm{C}$ über Nacht getempert. Danach wurden $5 \mu \mathrm{L}$ einer 2,5 \% Lösung des Blockcopolymers auf das behandelte Substrat getropft und wiederum für $1 \mathrm{~min}$ bei $2000 \mathrm{rpm}$ gespincoatet. Zum Schluss wurde die Probe im Vakuumofen bei $160^{\circ} \mathrm{C}$ über Nacht getempert. Für alle Polymerproben wurde Propylen-glykol-monomethyl-ether-acetat als Lösungsmittel verwendet.

Für die Vernetzungsversuche wurden die Proben für ein bestimmtes Zeitintervall mit UV-Licht einer bestimmten Wellenlänge $(\lambda>310 \mathrm{~nm}$ oder $\lambda=254 \mathrm{~nm})$ bestrahlt. Dafür wurde die Bestrahlungskammer UVACUBE inert von der Firma Hönle verwendet.

\section{Messprobleme bei erhöhten Temperaturen bei AFM-Untersuchungen}

Die Messung bei hohen Temperaturen bringt einige Messprobleme mit sich. Es ist schwierig mit dem AFM Aufnahmen der Höhenprofile mit einer guten Auflösung bei hohen Temperaturen zu erhalten, da der Cantilever bei erhöhten Temperaturen aufgrund thermischen Rauschens zum Schwingen gebracht wird. Die Messung wird durch das Rauschen beeinflusst. Die mittlere Auslenkung $\bar{z}$ des Cantilever wird laut Theorie wie folgt beschrieben:186

$$
\bar{z}=\sqrt{\frac{k_{\mathrm{B}} T}{k_{\mathrm{c}}}}
$$

Hierbei ist $k_{\mathrm{B}}$ die Boltzmannkonstante, $T$ die Umgebungstemperatur und $k_{\mathrm{c}}$ die Federkonstante des Cantilevers. Es wird deutlich, dass ein Cantilever mit einer großen Federkonstante weniger durch thermisches Rauschen zum Schwingen gebracht wird als ein Cantilever mit einer geringeren Federkonstante. Zusätzlich 
werden die Polymerproben mit zunehmender Temperatur sehr weich und können klebrig werden. Dies stört insofern den Messvorgang, da der Cantilever nicht mit einer beliebigen Amplitude schwingen kann. Ist die Probe so klebrig, dass der Cantilever selbst bei maximaler Amplitude nicht die Probenoberfläche verlassen kann, so ist es nicht möglich die Probe weiterhin zu vermessen.

\section{Scanrichtung und Auflösungslimit durch Cantileverspitze}

Die Bewegung der Cantilverspitze über die Probenoberfläche wird in Abbildung 6-1 schematisch dargestellt, wobei die grünen Linien den Hinweg und die orangen Linien den Rückweg der Spitze kennzeichnen. Die Spitze fährt sinusförmig an die Oberfläche heran und rastert die Probe zeilenweise ab. Ein Bild entsteht dabei aus den Daten einer Richtung, wobei in der vorliegenden Arbeit die Messdaten der Rückrichtung verwendet wurden. Die Abbildung 6-1 verdeutlicht, dass die Bewegung der Cantileverspitze in horizontaler Richtung wesentlich schneller ist als in vertikaler Richtung, wodurch die horizontale Richtung als schnelle Messrichtung

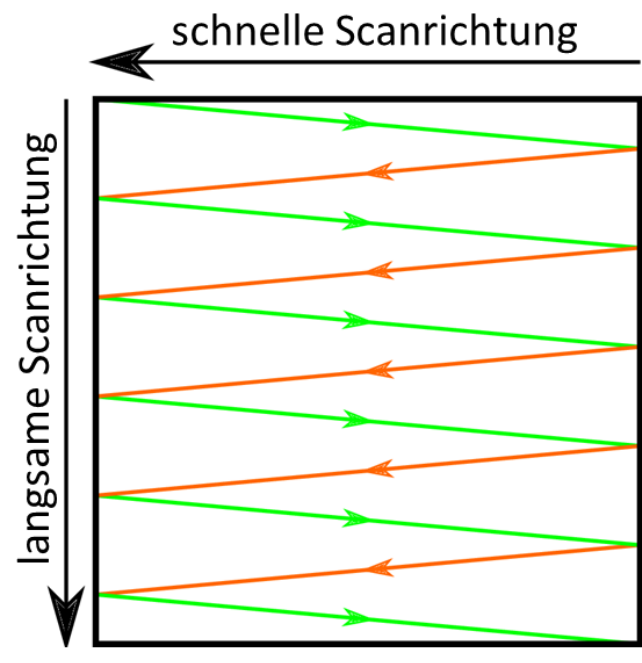

Abbildung 6-1 Schnelle und langsame Scanrichtung der Cantileverspitze. bezeichnet wird und die vertikale als langsame Richtung.

Ein weiteres Messproblem stellt der Radius der Cantileverspitze dar, da dieser die Auflösung des AFMs begrenzt. Dabei hängt es davon ab, wie nah die Objekte auf der Oberfläche nebeneinander liegen, wie hoch die Objekte sind und wie groß die Steigung am Rand der Erhebung ist. In Abbildung 6-2 soll dieses Problem verdeutlicht werden, indem eine Cantileverspitze über eine Reihe hoher Säulen sowie steiler Kanten fahren soll. Der Radius der gewählten Cantileverspitze ist zu groß für die in Abbildung 6-2 dargestellten Säulen, wodurch die Spitze nicht in den Zwischenraum eindringen kann und somit ein

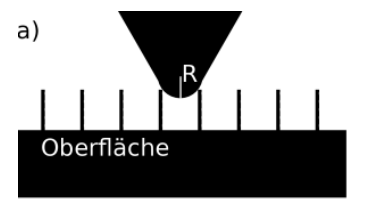

Abbild:

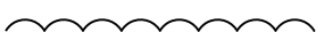

Abbildung 6-2 Darstellung vom Abrastern einer Probenoberfläche mit einer $\mathrm{zu}$ groß gewählten Cantileverspitze: a) Anreihung hoher Säulen; b) steile Kante. 
verfälschtes Höhenprofil entsteht. Allgemein gilt die Regel, dass Objekte in der Größenordnung des Spitzenradius nicht korrekt abgebildet werden können. Weiterhin ist auf den Einfluss der Spitzenform auf die Topographie von steilen Kanten zu achten.

\section{Kalibrierung 122,177}

In Abschnitt 2.7.3 wurde bereits beschrieben, dass mit dem AFM verschiedene mechanische Eigenschaften der Polymerproben untersucht werden können. Dafür wird das AFM mit dem PeakForce-QNMTM${ }^{\mathrm{T}}$-Modus betrieben, bei dem für jeden Bildpunkt eine komplette Kraft-Abstandskurve aufgenommen wird. Aus den Kurven können dann die Eigenschaften wie z. B. DMT-Modul bestimmt werden. Um verlässliche Daten ermitteln zu können, muss eine Kalibrierung des Systems an einem Standard mit bekannten E-Moduln erfolgen. Bei der Standardprobe handelte es sich um ein PS-LDPE-Polymerblend der Firma Veeco.

Im ersten Schritt der Kalibrierung wird die Deflection Sensitivity des Cantilevers bestimmt. Zur Bestimmung eines genauen Wertes der Deflection Sensitivity muss das exakte Modulverhältnis beider Polymere eingestellt werden. Dazu wurde die Differenz der logarithmierten DMT-Moduln von PS (1960 GPa, $\log E_{\mathrm{DMT}}=9,29 \mathrm{~Pa}$ ) und LDPE $\left(84,2 \mathrm{GPa}, \log E_{\mathrm{DMT}}=7,93 \mathrm{~Pa}\right) \mathrm{zu} \Delta \log D M T=1,36$ ermittelt. Es wurden Bilder der Probe in einer Auflösung 128 x 128 Pixel aufgenommen und mit Hilfe der Software NanoScope Analysis konnte die Differenz des $\log E_{\mathrm{DMT}}$ bestimmt werden (Abbildung 6-3). Der Wert der Deflection Sensitivity wurde solange variiert, bis der Wert des $\log E_{\mathrm{DMT}}$ im Bereich von 1,36 \pm 0,02 lag. Abbildung 6-3 zeigt das Profil (links) und die Verteilungskurve (rechts) für das $\log E_{\mathrm{DMT}}$ der Standard-Kalibrierprobe. 

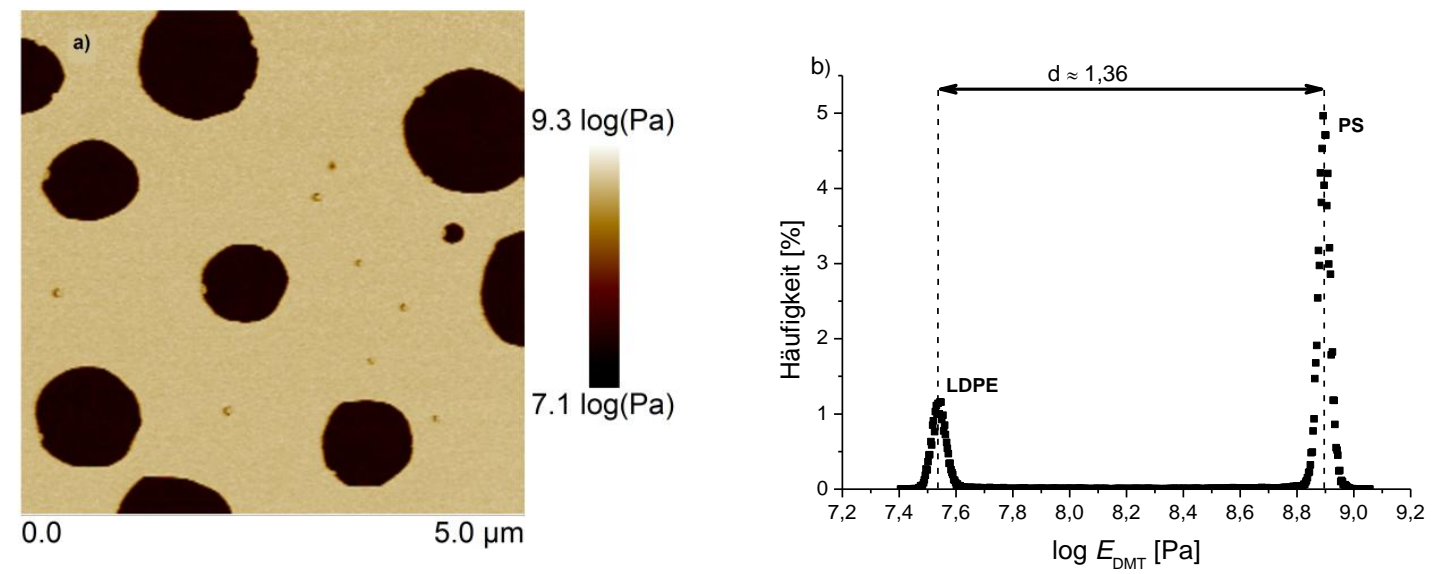

Abbildung 6-3 a) Profil des logarithmierten DMT-Moduls der PS-LDPE-Standardprobe; b) Verteilungskurve des logarithmierten DMT-Moduls.

Im Anschluss wird der Cantilever von der Oberfläche weggefahren, um mit Hilfe des Thermal Tune-Modus die exakte Federkonstante $k_{\text {Cantilever }}$ bestimmen zu können.

Im letzten Schritt der Kalibrierung muss noch der Spitzenradius R ermittelt werden. Dies erfolgte mit folgender Rechnung:

$$
E_{\text {rel }} \propto \frac{k_{\text {Cantilever }}^{*}}{\sqrt{R^{*}}}
$$

$E_{\text {rel }}$ steht hier für den relativen reduzierten E-Modul und die Sternchen sind zur besseren Orientierung da. Für den tatsächlichen reduzierten E-Modul $E_{\mathrm{r}}$ gilt ebenfalls:

$$
E_{\mathrm{r}} \propto \frac{k_{\text {Cantilever }}}{\sqrt{R}}
$$

Beide Moduln werden in Relation zueinander gesetzt:

$$
\left(\frac{E_{\mathrm{rel}}}{E_{\mathrm{r}}}\right)^{2}=\left(\frac{k_{\text {Cantilever }}^{*}}{k_{\text {Cantilever }}}\right)^{2} \cdot \frac{R}{R^{*}}
$$

Mit der exakten Deflection Sensitivity sowie der exakten Federkonstante wird nun das DMT-Profil des Standards aufgenommen und mit der Software ausgewertet. Dabei erhält man den tatsächlich reduzierten E-Modul $E_{\mathrm{r}}$ und kann diesen in 
folgender umgestellter Formel einsetzen, um somit den exakten Spitzenradius zu berechnen:

$$
R=R^{*} \cdot\left(\frac{E_{\mathrm{rel}}}{E_{\mathrm{r}}}\right)^{2} \cdot\left(\frac{k_{\text {Cantilever }}}{k_{\text {Cantilever }}^{*}}\right)^{2}
$$

Die Kalibrierung ist mit der Ermittlung der exakten Deflection Sensitivity, der korrekten Federkonstante sowie des genauen Spitzenradius beendet und ausgesuchte Proben können nun untersucht werden (siehe Abschnitt 2.7.3).

\subsubsection{Probenpräparation für UV/Vis}

Einige Tropfen einer 2,5\% Polymerlösung wurden auf Glasplättchen, welche vorher in einer Plasmaanlage gereinigt wurden, aufgetragen. Anschließend wurde das Lösungsmittel THF im Vakuumofen bei $40^{\circ} \mathrm{C}$ abgedampft. Für die Bestrahlung der Proben mit UV-Licht wurde die UV-Bestrahlungskammer UVACUBE verwendet. Die Proben wurden für ein bestimmtes Zeitintervall mit UV-Licht einer bestimmten Wellenlänge $(\lambda>310 \mathrm{~nm}$ und $\lambda=254 \mathrm{~nm})$ bestrahlt.

\subsubsection{Durchführung der DMA-Experimente}

\section{Biegebalkenexperiment}

In diesem Experiment wird eine Seite der Probe fest eingespannt, während die andere Seite der Probe in dem beweglichen Probenstab eingespannt ist. Ein Vorteil dieser Methode besteht in der Verwendung sog. Materialpockets für die weichen, klebrigen bis pulverförmigen Polymerproben, die ohne Unterstützung nicht einzuspannen wären. Das Probenmaterial wird in einen Umschlag aus rostfreiem Stahl gegeben. Dieser Umschlag wird zusammengefaltet und in die Apparatur eingebaut. Da Stahl einen konstanten Modul und einen konstanten Verlust in den untersuchten Temperaturbereichen aufweist, können alle gemessenen Änderungen in den mechanischen Eigenschaften auf das Probenmaterial zurückgeführt werden. ${ }^{187}$ Für die Untersuchungen wurden $20 \mathrm{mg}$ bis $70 \mathrm{mg}$ Probenmaterial verwendet. Die Messungen wurden mit einer Verschiebungsamplitude von 0,02 mm und einer Frequenz von $5 \mathrm{~Hz}$ durchgeführt. Die Proben wurden in einem Temperaturbereich von $-80^{\circ} \mathrm{C}$ bis $250{ }^{\circ} \mathrm{C}$ mit einer Heizrate von $2 \mathrm{~K} / \mathrm{min}$ gemessen. Die Werte für die Glasübergangstemperaturen wurden aus dem jeweiligen Wendepunkt der Stufe des Verlustmoduls bestimmt. 


\section{Zugversuch}

Für die Messung der Absolutwerte des Moduls wurde die Zuggeometrie verwendet. Für die Messung mussten dünne Polymerfilme hergestellt werden. Somit wurde das synthetisierte Polymer zunächst in THF gelöst und im Anschluss wurde das Lösungsmittel im Vakuumofen bei $40^{\circ} \mathrm{C}$ entfernt. Für die Messungen war es wichtig, dass die dünnen Polymerfolien homogen waren. Aus den Folien wurden rechteckige Bänder herausgeschnitten, die eine Breite von 2 bis $5 \mathrm{~mm}$ und eine Dicke von ca. 0,1 mm hatten. Die Längen betrugen zwischen 4 und $10 \mathrm{~mm}$. Für die Messungen wurden die dünnen Polymerfolien in einem beweglichen Probenstab eingespannt. Der Stab ist frei gelagert und an seinem hinteren Ende mit einem Kraftmotor verbunden, welcher aus einer Spule und einem Magnet besteht. Die Auslenkung des Probenstabs wurde mithilfe eines LVDT (Linear Variable Differential Transformator) gemessen. Dabei handelt es sich um ein abgeglichenes Spulenpaar, welches den Probenstab umschließt und dessen Bewegung einen Spannungspuls im Spulensystem zur Folge hat. Zum Einspannen wurde eine Vorspannung von -0,2 N verwendet, damit die Proben im Zugversuch keine Beulen werfen. Die Messungen wurden mit einer Scheramplitude von 0,1\% und bei einer Frequenz von $1 \mathrm{~Hz}$ durchgeführt.

Für die Untersuchung der Vernetzung und den damit verbundenen Einfluss auf den Modul wurden die Proben im Zugversuch untersucht und mit UV-Licht einer bestimmten Wellenlänge $(\lambda>310 \mathrm{~nm})$ bestrahlt. Dafür wurde die UV-Lampe XH 100 der Firma Müller verwendet. Vor Beginn der Bestrahlung wurde 5 min lang eine dynamisch mechanische Messung durchgeführt. Danach wurde die Probe für $1 \mathrm{~h}$ mit UV-Licht mit der Wellenlänge $\lambda>310 \mathrm{~nm}$ bestrahlt und anschließend erneut für 5 min dynamisch gemessen. Dieser Ablauf wurde mehrfach wiederholt, sodass sich Änderungen im Modul in Abhängigkeit der Bestrahlungsdauer untersuchen ließen. 


\section{Literaturverzeichnis}

(1) Plastics - the Facts 2013 An analysis of European latest plastics production, demand and waste data; Brüssel, Belgien, 2013; pp. 1-37.

(2) Müller, A. H. E.; Matyjaszewski, K. Controlled and Living Polymerizations; 2009; p. 612.

(3) Staudinger, H. Berichte d. Dtsch. Chem. Gesellschaft 1920, 59, 1073-1085.

(4) Staudinger, H. Berichte d. Dtsch. Chem. Gesellschaft 1926, 59, 3019-3043.

(5) Staudinger, H.; Brunner, M.; Frey, K.; Garbsch, P.; Signer, R.; Wehrli, S. Berichte d. Dtsch. Chem. Gesellschaft 1929, 59, 241-263.

(6) Braun, D. Int. J. Polym. Sci. 2009, 2009, 1-10.

(7) Schubert, U. Chemie unserer Zeit 2005, 39, 3.

(8) Fraunhofer IAP Jahresbericht 2012; 2012; pp. 1-136.

(9) Matyjaszewski, K.; Xia, J. Chem. Rev. 2001, 101, 2921-2990.

(10) Szwarc, M. Nature 1956, 178, 1168-1169.

(11) Szwarc, M.; Levy, M.; Milkovich, R. J. Am. Chem. Soc. 1956, 78, 2656-2657.

(12) Chiefari, J.; Chong, Y. K. B.; Ercole, F.; Krstina, J.; Jeffery, J.; Le, T. P. T.; Mayadunne, R. T. A.; Meijs, G. F.; Moad, C. L.; Moad, G.; Rizzardo, E.; Thang, S. H.; South, C. Macromolecules 1998, 31, 5559-5562.

(13) Förster, S.; Plantenberg, T. Angew. Chem. Int. Ed. Engl. 2002, 41, 689-714.

(14) Schacher, F. H.; Rupar, P. a; Manners, I. Angew. Chem. Int. Ed. Engl. 2012, 51, 7898-7921.

(15) Seki, T. Bull. Chem. Soc. Jpn. 2007, 80, 2084-2109.

(16) Boschmann, D.; Vana, P. Macromolecules 2007, 40, 2683-2693.

(17) Boyer, C.; Valade, D.; Sauguet, L.; Ameduri, B.; Boutevin, B. Macromolecules 2005, 38, 10353-10362. 
(18) Lechner, M. D.; Gehrke, K.; Nordmeier, E. H. Makromolekulare Chemie: Ein Lehrbuch für Chemiker, Physiker, MAterialwissenschaftler und Verfahrenstechniker; Birkhäuser Verlag, 2010.

(19) Jenkins, A. D.; Jones, R. G.; Moad, G. Pure Appl. Chem. 2009, 82, 483-491.

(20) Webster, 0. W. Science (80-. ). 1991, 251, 887-893.

(21) Moad, G.; Rizzardo, E.; Solomon, D. H. Macromolecules 1982, 15, 909-914.

(22) Moad, G.; Rizzardo, E.; Solomon, O. H.; Organic, A. Polym. Bull. 1982, 6, 589593.

(23) Hawker, C. J.; Bosman, a W.; Harth, E. Chem. Rev. 2001, 101, 3661-3688.

(24) Solomon, D. H.; Rizzardo, E.; Cacioli, P. Polymerization Process and Polymers Produced Thereby. US Patent 4581429, 1986.

(25) Kato, M.; Kamigaito, M.; Sawamoto, M.; Higashimuras, T. Macromolecules 1996, 28, 1721-1723.

(26) Wang, J.; Matyjaszewski, K. J. Am. Chem. Soc. 1995, 117, 5614-5615.

(27) Moad, G.; Rizzardo, E.; Thang, S. H. Aust. J. Chem. 2006, 59, 669.

(28) Moad, G.; Rizzardo, E.; Thang, S. H. Aust. J. Chem. 2009, 62, 1402.

(29) Lutz, J.-F. Polym. Int. 2006, 55, 979-993.

(30) Gao, H.; Matyjaszewski, K. Prog. Polym. Sci. 2009, 34, 317-350.

(31) Moad, G.; Rizzardo, E.; Thang, S. H. Aust. J. Chem. 2005, 58, 379.

(32) Moad, G.; Rizzardo, E.; Thang, S. H. Aust. J. Chem. 2006, 59, 669.

(33) Moad, G.; Rizzardo, E.; Thang, S. H. Chem. Asian J. 2013, 8, 1634-1644.

(34) Barner-kowollik, C.; Perrier, S. J. Polym. Sci. Part A Polym. Chem. 2008, 46, 5715-5723.

(35) Arita, T.; Buback, M.; Janssen, O.; Vana, P. Macromol. Rapid Commun. 2004, 25, 1376-1381.

(36) Arita, T.; Buback, M.; Vana, P. Macromolecules 2005, 38, 7935-7943.

(37) Ullal, C. K.; Primpke, S.; Schmidt, R.; Böhm, U.; Egner, A.; Vana, P.; Hell, S. W. Macromolecules 2011, 44, 7508-7510. 
(38) Chong, B. Y. K.; Le, T. P. T.; Moad, G.; Rizzardo, E.; Thang, S. H. Macromolecules 1999, 32, 2071-2074.

(39) Barner, L.; Davis, T. P.; Stenzel, M. H.; Barner-Kowollik, C. Macromol. Rapid Commun. 2007, 28, 539-559.

(40) Boyer, C.; Stenzel, M. H.; Davis, T. P. J. Polym. Sci. Part A Polym. Chem. 2011, 49, 551-595.

(41) Yang, P.; Ratcli, L. P. D.; Armes, S. P. Macromolecules 2013, 46, 8545-8556.

(42) Arita, T.; Beuermann, S.; Buback, M.; Vana, P. Macromol. Mater. Eng. 2005, 290, 283-293.

(43) Wang, X.; Li, S.; Su, Y.; Huo, F.; Zhang, W. J. Polym. Sci. Part A Polym. Chem. 2013, 51, 2188-2198.

(44) Chakrabarty, A.; Singha, N. K. J. Colloid Interface Sci. 2013, 408, 66-74.

(45) Zhang, W.; Agosto, F. D.; Boyron, O.; Rieger, J.; Charleux, B. Macromolecules 2011, 44, 7584-7593.

(46) Chiefari, J.; Mayadunne, R. T. A.; Moad, C. L.; Moad, G.; Rizzardo, E.; Postma, A.; Skidmore, M. A.; Thang, S. H. Macromolecules 2003, 36, 2273-2283.

(47) Chong, B. Y. K.; Krstina, J.; Le, T. P. T.; Moad, G.; Postma, A.; Rizzardo, E.; Thang, S. H. Macromolecules 2003, 36, 2256-2272.

(48) Rizzardo, E.; Chen, M.; Chong, B.; Moad, G.; Skidmore, M.; Thang, S. H. Macromol. Symp. 2007, 248, 104-116.

(49) Li, C.; Benicewicz, B. C. J. Polym. Sci. Part A Polym. Chem. 2005, 43, 15351543.

(50) Skey, J.; O’Reilly, R. K. Chem. Commun. (Camb). 2008, 4183-4185.

(51) Ham, M.; HoYouk, J.; Kwon, Y.-K.; Kwark, Y.-J. J. Polym. Sci. Part A Polym. Chem. 2012, 50, 2389-2397.

(52) Perrier, S.; Takolpuckdee, P. J. Polym. Sci. Part A Polym. Chem. 2005, 43, 5347-5393.

(53) McLeary, J. B.; Calitz, F. M.; McKenzie, J. M.; Tonge, M. P.; Sanderson, R. D.; Klumperman, B. Macromolecules 2004, 37, 2383-2394.

(54) Mcleary, J. B.; Calitz, F. M.; Mckenzie, J. M.; Tonge, M. P.; Sanderson, R. D.; Klumperman, B. Macromolecules 2005, 38, 3151-3161. 
(55) Arita, T.; Beuermann, S.; Buback, M.; Vana, P.; Chemie, P.; Göttingen, G. $e$ Polymers 2004, 1-14.

(56) Barner-Kowollik, C.; Coote, M. L.; Davis, T. P.; Radom, L.; Vana, P. J. Polym. Sci. Part A Polym. Chem. 2003, 41, 2828-2832.

(57) Davis, T. P.; Matyjaszewski, K. In Handbook of Radical Polymerization; Hoboken: Wiley-Interscience, 2002.

(58) Toy, A. A.; Vana, P.; Davis, T. P.; Barner-kowollik, C. Macromolecules 2004, $37,744-751$.

(59) Kwak, Y.; Goto, A.; Fukuda, T. Macromolecules 2004, 37, 1219-1225.

(60) Monteiro, M. J.; Brouwer, H. De. Macromolecules 2001, 34, 349-352.

(61) Kwak, Y.; Goto, A.; Tsujii, Y.; Murata, Y.; Komatsu, K.; Fukuda, T. Macromolecules 2002, 35, 3026-3029.

(62) Arita, T.; Buback, M.; Vana, P. Macromolecules 2005, 38, 7935-7943.

(63) Buback, M.; Vana, P. Macromol. Rapid Commun. 2006, 27, 1299-1305.

(64) Buback, M.; Janssen, O.; Oswald, R.; Schmatz, S.; Vana, P. Macromol. Symp. 2007, 248, 158-167.

(65) Matyjaszewski, K.; Davis, T. P. In Handbook of Radical Polymerization; Matyjaszewski, K., Ed.; Hoboken: Wiley-Interscience, 2002.

(66) Barner-Kowollik, C.; Vana, P.; Davis, T. P. In Handbook of Radical Polymerization; Matyjaszewski, K.; Davis, T. P., Eds.; Hoboken: WileyInterscience, 2002.

(67) Hutchinson, R. A.; Aronson, M. T.; Richards, J. R. Macromolecules 1993, 26, 6410-6415.

(68) Grubsici, Z.; Rempp, P.; Benoit, H. J. Polym. Sci. Part B Polym. Lett. 1967, 5, 753-759.

(69) Spatorico, A. L.; Coulter, B. J. Polym. Sci. Polym. Phys. Ed. 1973, 11, 11391150.

(70) Reed, W. F. Macromol. Chem. Phys. 1995, 196, 1539-1575.

(71) Wyatt, P. J. Anal. Chim. Acta 1993, 272, 1-40.

(72) Bates, F. S.; Fredrickson, G. H. Phys. Today 1999, 52, 32. 
(73) Ruzette, A.-V.; Leibler, L. Nat. Mater. 2005, 4, 19-31.

(74) Han, C. D.; Kim, J.; Kim, J. K. Macromolecules 1989, 22, 383-394.

(75) Khandpur, A. K.; Förster, S.; Bates, F. S.; Hamley, I. W.; Ryan, A. J. Macromolecules 1995, 28, 8796-8806.

(76) Förster, S.; Antonietti, M. Adv. Mater. 1998, 10, 195-217.

(77) Elias, H.-G. An Introduction to Polymer Science; 1997; p. 492.

(78) Strobl, G. The Physics of Polymers: Concepts for understanding their structures and behaviour; Springer, 1997.

(79) Mai, Y.; Eisenberg, A. Chem. Soc. Rev. 2012, 41, 5969-5985.

(80) Lee, K.-S.; Kobayashi, S. Block-Copolymers, Nanocomposittes, Organic/Inorganic Hybrids, Polymethylenes; Springer, 2010.

(81) Meier, D. J.; Company, S. D. J. Polym. Sci. Part C 1969, 26, 81-98.

(82) Bates, F. S.; Fredrickson, G. H. Annu. Rev. Phys. Chem. 1990, 41, 525-557.

(83) Helfand, E. Macromolecules 1975, 8, 552-556.

(84) Helfand, E.; Wasserman, Z. R. Macromol. Symp. 1976, 9, 879-888.

(85) Helfand, E.; Wasserman, Z. R. Macromolecules 1978, 11, 960-966.

(86) Helfand, E.; Wasserman, Z. R. Macromolecules 1980, 13, 994-998.

(87) Leibler, L. Macromolecules 1980, 13, 1602-1617.

(88) Matsen, M. W.; Bates, F. S. Macromolecules 1996, 29, 1091-1098.

(89) Matsen, M. W.; Bates, F. S. J. Chem. Phys. 1997, 106, 2436.

(90) Fredrickson, G. H.; Helfand, E. J. Chem. Phys. 1987, 87, 697.

(91) Semenov, A. N. Sov. Phys. JETP 1985, 61, 733-742.

(92) Ohta, T.; Kawasaki, K. Macromolecules 1986, 19, 2621-2632.

(93) Matsen, M. W.; Bates, F. S. Macromolecules 1996, 29, 1091-1098.

(94) Matsen, M. W.; Schick, M. Phys. Rev. Lett. 1994, 72, 2660-2663. 
(95) Holoubek, J.; Baldrian, J.; Lednický, F.; Lal, J. Polym. Int. 2009, 58, 81-87.

(96) Hasegawa, H.; Tanaka, H.; Hashimoto, T.; Han, C. C. Macromolecules 1987, 20, 2120-2127.

(97) Gopinathan, A. Phys. Rev. E 2005, 71, 041601.

(98) Zehner, R. W.; Lopes, W. A.; Morkved, T. L.; Jaeger, H.; Sita, L. R. Langmuir 1998, 14, 1401-1404.

(99) Vernon-Parry, K. D. III-Vs Rev. 2000, 13, 40-44.

(100) Magonov, S. N.; Reneker, D. H. Annu. Rev. Mater. Sci. 1997, 27, 175-222.

(101) Sriprom, W.; James, M.; Perrier, S.; Neto, C. Macromolecules 2009, 42, 31383146.

(102) Ji, S.; Liu, C.-C.; Son, J. G.; Gotrik, K.; Craig, G. S. W.; Gopalan, P.; Himpsel, F. J.; Char, K.; Nealey, P. F. Macromolecules 2008, 41, 9098-9103.

(103) Stehlin, F.; Diot, F.; Gwiazda, A.; Dirani, A.; Salaun, M.; Zelsmann, M.; Soppera, 0. Langmuir 2013, 29, 12796-12803.

(104) Rasmont, a; Leclère, P.; Doneux, C.; Lambin, G.; Tong, J.; Jérôme, R.; Brédas, J.; Lazzaroni, R. Colloids Surf. B. Biointerfaces 2000, 19, 381-395.

(105) Ahn, H.; Ryu, D. Y.; Kim, Y.; Kwon, K. W.; Lee, J.; Cho, J. Macromolecules 2009, 42, 7897-7902.

(106) Hajduk, D. A.; Gruner, S. M.; Erramilli, S.; Register, R. A. Macromolecules 1996, 29, 1473-1481.

(107) Khandpurj, A. K.; Förster, S.; Bates, F. S.; Hamley, I. W.; Ryan, A. J. Macromolecules 1995, 28, 8796-8806.

(108) Zhao, Y.; Saijo, K.; Hashimoto, T. Macromolecules 2013, 46, 957-970.

(109) Sriprom, W.; Neel, M.; Gabbutt, C. D.; Heron, B. M.; Perrier, S. J. Mater. Chem. 2007, 17, 1885.

(110) Corredor, C. C.; Huang, Z.-L.; Belfield, K. D.; Morales, A. R.; Bondar, M. V. Chem. Mater. 2007, 19, 5165-5173.

(111) Wrana, C. Polymerphysik- Eine physikalische Beschreibung von Elastomeren und ihren anwendungsrelevanten Eigenschaften; Springer Spektrum, 2014; p. 339. 
(112) Binnig, G.; Quate, C. F.; Gerber, C. Phys. Rev. Lett. 1986, 56, 930-933.

(113) Schön, P.; Bagdi, K.; Molnár, K.; Markus, P.; Pukánszky, B.; Julius Vancso, G. Eur. Polym. J. 2011, 47, 692-698.

(114) Young, T. J.; Monclus, M. a; Burnett, T. L.; Broughton, W. R.; Ogin, S. L.; Smith, P. a. Meas. Sci. Technol. 2011, 22, 125703.

(115) Zhao, B.; Song, Y.; Wang, S.; Dai, B.; Zhang, L.; Dong, Y.; Lü, J.; Hu, J. Soft Matter 2013, 9, 8837.

(116) Adamcik, J.; Berquand, A.; Mezzenga, R. Appl. Phys. Lett. 2011, 98, 193701.

(117) Veeco Instruments Inc. Multimode 8 Instr. Man. A - Chapter 9 Contact AFM Mode; 2010; p. 149.

(118) Kaemmer, S. B. Introduction to Bruker's ScanAsyst and PeakForce Tapping AFM Technology; Bruker application note AN133, Rev. A0; Application Note \#133; 2011; pp. 1-12.

(119) Veeco Instruments Inc. Multimode 8 Instr. Man. A - Chapter 10 Tapping Mode AFM; 2010; p. 167.

(120) Veeco Instruments Inc. Multimode 8 Instr. Man. A - Chapter 8 ScanAsyst; 2010; p. 119.

(121) Pittenger, B.; Erina, N.; Su, C. Quantitative Mechanical Property Mapping at the Nanoscale with PeakForce QNM; Bruker application note AN128, Rev. B0; 2012; pp. 1-12.

(122) Veeco Instruments Inc. PeakForce QNM User Guid. - Chapter 4 Calibration; 2010 ; p. 53.

(123) Elson, J. M.; Bennett, J. M. Appl. Opt. 1995, 34, 201-208.

(124) Sidick, E. Proc. SPIE 2009, 7390, 73900L-73900L-12.

(125) Brigham, E. O. FFT: Schnelle Fourier-Transformation; Oldenbourg, 1982.

(126) Mack, C. a. J. Micro/Nanolithography, MEMS, MOEMS 2011, 10, 040501.

(127) Trenor, S. R.; Shultz, A. R.; Love, B. J.; Long, T. E. Chem. Rev. 2004, 104, 30593077.

(128) Minsk, L. M.; Smith, J. G.; Van Deusen, W. P.; Wright, J. F. J. Appl. Polym. Sci. 1959, 11, 302-307. 
(129) Deusen, W. P. V. A. N.; Minsk, M.; Company, E. K.; York, N. J. Appl. Polym. Sci. 1959, 11, 308-311.

(130) Coqueret, X. Macromol. Chem. Phys. 1999, 200, 1567-1579.

(131) Coleman, M. M.; Hu, Y. I. N.; Sobkowiak, M.; Painter, P. C. J. Polym. Sci. Part B Polym. Phys. 1997, 36, 1579-1590.

(132) Ihmels, H. Tetrahedron Lett. 1998, 39, 8641-8642.

(133) Shindo, Y.; Katagirit, N.; Ebisunol, T.; Hasegawal, M.; Mitsudan, M. Angew. Makromol. Chemie 1996, 240, 231-239.

(134) Cockburn, E. S.; Davidson, R. S.; Pratt, J. E. J. Photochem. Photobiol. A Chem. 1996, 94, 83-88.

(135) Vo, C. D.; Kuckling, D.; Adler, H.-J. P.; Schönhoff, M. Colloid Polym. Sci. 2002, 280, 400-409.

(136) Berger, J.; Zweifel, H. Angew. Makromol. Chemie 1983, 115, 163-181.

(137) Yourick, J. J.; Bronaugh, R. L. J. Appl. Toxicol. 1997, 17, 153-158.

(138) Born, S. L.; Hu, J. K.; Lehman-mckeeman, L. D. Drug Metab. Dispos. 2000, 28, 218-223.

(139) Ong, E. B. B.; Watanabe, N.; Saito, A.; Futamura, Y.; Abd El Galil, K. H.; Koito, A.; Najimudin, N.; Osada, H. J. Biol. Chem. 2011, 286, 14049-14056.

(140) Lin, P.-H.; Ke, Y.-Y.; Su, C.-T.; Shiao, H.-Y.; Hsieh, H.-P.; Chao, Y.-K.; Lee, C.-N.; Kao, C.-L.; Chao, Y.-S.; Chang, S.-Y.J. Virol. 2011, 85, 9114-9126.

(141) Wheelock, C. E. J. Am. Chem. Soc. 1959, 81, 1348-1352.

(142) Jones, G.; Jackson, W. R.; Choi, C.; Bergmark, W. R. J. Phys.Chem. 1985, 89, 294-300.

(143) Hoshiyama, M.; Kubo, K.; Igarashi, T.; Sakurai, T. J. Photochem. Photobiol. A Chem. 2001, 138, 227-233.

(144) Jones, G.; Rahman, M. A. J. Phys.Chem. 1994, 98, 13028-13037.

(145) Tsukagoshi, K.; Tanabe, J.; Yagi, I.; Shigeto, K.; Yanagisawa, K.; Aoyagi, Y. J. Appl. Phys. 2006, 99, 064506.

(146) Ciamician, G.; Silber, P. Ber. Dtsch. Chem. Ges. Berlin 1902, 35, 4128-4131. 
(147) Krauch, C. H.; Farid, S.; Schenck, G. O. Chem. Ber. 1966, 99, 625-633.

(148) D’Auria, M.; Racioppi, R. J. Photochem. Photobiol. A Chem. 2004, 163, 557559.

(149) Lewis, F. D.; Barancyk, S. V. J. Am. Chem. Soc. 1989, 111, 8653-8661.

(150) Schenck, G. O.; Wilucki, I. V. O. N.; Krauch, C. H. Chem. Ber. 1962, 95, 14091412.

(151) Wolff, T.; Görner, H. Phys. Chem. Chem. Phys. 2004, 6, 368-376.

(152) Brett, T. J.; Alexander, J. M.; Stezowski, J. J. J. Chem. Soc. Perkin Trans. 2 2000, 1105-1111.

(153) Chen, Y.; Geh, J.-L. Polymer (Guildf). 1996, 37, 4481-4486.

(154) Yonezawa, N.; Yoshida, T.; Hasegawa, M. J. Chem. Soc. Perkin Trans I 1983, 1083-1086.

(155) Chen, Y.; Geh, J.-L. Polymer (Guildf). 1996, 37, 4473-4480.

(156) Chen, Y. U. N.; Wu, J. J. Polym. Sci. Part A Polym. Chem. 1994, 32, 1867-1875.

(157) Chen, Y. U. N.; Chou, C. J. Polym. Sci. Part A Polym. Chem. 1995, 33, 27052714.

(158) Jiang, J.; Qi, B.; Lepage, M.; Zhao, Y. Macromolecules 2007, 40, 790-792.

(159) Sinkel, C.; Greiner, A.; Agarwal, S. Macromol. Chem. Phys. 2010, 211, 18571867.

(160) Huyck, R. H.; Trenor, S. R.; Love, B. J.; Long, T. E. J. Macromol. Sci. Part A 2007, $45,9-15$.

(161) He, J.; Tong, X.; Tremblay, L.; Zhao, Y. Macromolecules 2009, 42, 7267-7270.

(162) Jiang, J.; Qi, B.; Lepage, M.; Zhao, Y. Macromolecules 2007, 40, 790-792.

(163) Förster, N. Nano-Carrier Synthesis via Z-RAFT Star Polymerisation, 2012.

(164) Immergut, E. H.; Brandrup, J. Polymer Handbook; 1989.

(165) Gaur, U.; Wunderlich, B. Macromolecules 1980, 13, 1618-1625. 
(166) Riesen, R.; Schawe, J. Die Glasübergangstemperatur gemessen mit verschiedenen TA-Techniken, Teil 2: Ermittlung der Glasübergangstemperaturen; 2003; pp. 1-20.

(167) Knoll, A.; Magerle, R.; Krausch, G. J. Chem. Phys. 2004, 120, 1105-1116.

(168) Tsarkova, L.; Knoll, A.; Krausch, G.; Magerle, R.; Ii, P. C.; Uni, V. 2006, 36083615.

(169) Anastasiadis, S. H.; Russell, T. P.; Satija, S. K.; Majkrzak, C. F. Phys. Rev. Lett. 1989, 62, 1852-1855.

(170) Park, I.; Park, S.; Park, H.; Chang, T. Macromolecules 2006, 39, 315-318.

(171) Mansky, P. Science (80-. ). 1997, 275, 1458-1460.

(172) In, I.; La, Y.-H.; Park, S.-M.; Nealey, P. F.; Gopalan, P. Langmuir 2006, 22, 7855-7860.

(173) Han, E.; In, I.; Park, S.-M.; La, Y.-H.; Wang, Y.; Nealey, P. F.; Gopalan, P. Adv. Mater. 2007, 19, 4448-4452.

(174) Stühn, B. J. Polym. Sci. Part B Polym. Phys. 1992, 30, 1013-1019.

(175) Holzer, B.; Lehmann, A.; Stühn, B. Polymer (Guildf). 1991, 32, 1935-1942.

(176) Swallowe, G. M.; Lee, S. F. J. Mater. Sci. 2006, 41, 6280-6289.

(177) Young, T. J.; Monclus, M. a; Burnett, T. L.; Broughton, W. R.; Ogin, S. L.; Smith, P. a. Meas. Sci. Technol. 2011, 22, 125703.

(178) Raaij, M. E. Van; Segers-nolten, I. M. J.; Subramaniam, V. Biophys. J. 2006, 91, L96-L98.

(179) Kumar E.K., P.; Feldborg, L. N.; Almdal, K.; Andresen, T. L. Chem. Mater. 2013, 25, 1496-1501.

(180) Moad, G.; Rizzardo, E.; Thang, S. H. Aust. J. Chem. 2009, 62, 1402.

(181) Moad, G.; Chong, Y. K.; Postma, A.; Rizzardo, E.; Thang, S. H. Polymer (Guildf). 2005, 46, 8458-8468.

(182) Polymer Standard Service GmbH.

(183) Beuermann, S.; Paquet, D. a.; McMinn, J. H.; Hutchinson, R. a. Macromolecules 1996, 29, 4206-4215. 
(184) Zhang, B.; Wang, F.; Yue, J. Synth. Commun. 2007, 37, 63-70.

(185) Shaughnessy, K.; Kim, P.; Hartwig, J. J. Am. Chem. Soc. 1999, 2123-2132.

(186) Meyer, E.; Hug, H.; Bennewitz, R. Scanning Probe Microscopy: the lab on the tip; Springer, 2004; pp. 0-224.

(187) Royall, P. G.; Huang, C.; Tang, S. J.; Duncan, J.; Van-de-Velde, G.; Brown, M. B. Int. J. Pharm. 2005, 301, 181-191. 


\section{Anhang A}

\section{Zusammensetzungen der Polymerisationsmischungen:}

Tabelle A-1 Homopolymerisation A: MMA (4,8 mol/L) mit dem RAFT-Agens (24 mmol/L) und AIBN $(5 \mathrm{mmol} / \mathrm{L})$ in DMF $(9 \mathrm{~mL})$ bei $70{ }^{\circ} \mathrm{C}$.

\begin{tabular}{|l|l|l|l|l|l|}
\hline Probe & Bedingung & Zeit [h] & Umsatz [\%] & $\begin{array}{l}\overline{\boldsymbol{M}}_{\mathbf{n}} \\
{[\mathbf{g} / \mathbf{m o l}]}\end{array}$ & $\boldsymbol{Ð}$ \\
\hline $\mathbf{4 3 - 2}$ & & 1 & 18 & 5456 & 1,4 \\
\hline $\mathbf{4 3 - 3}$ & & 2 & 32 & 8397 & 1,3 \\
\hline $\mathbf{4 3 - 4}$ & $\begin{array}{c}\text { MMA:RAFT:AIBN } \\
=\end{array}$ & 5,25 & 68 & 14700 & 1,21 \\
\hline $\mathbf{4 3 - 5}$ & $200: 1: 0,2$ & 6,5 & 75 & 17180 & 1,15 \\
\hline $\mathbf{4 3 - 6}$ & & 17 & 100 & 20100 & 1,16 \\
\hline $\mathbf{4 3 - 7}$ & & 8 & 82 & 16770 & 1,19 \\
\hline
\end{tabular}

Tabelle A-2 Copolymerisation A: MMA (5 mol/L) und CMA (5 mol\%) mit dem RAFT-Agens $(23 \mathrm{mmol} / \mathrm{L})$ und AIBN $(5 \mathrm{mmol} / \mathrm{L})$ in DMF $(5 \mathrm{~mL})$ bei $70^{\circ} \mathrm{C}$.

\begin{tabular}{|c|c|c|c|c|c|}
\hline Probe & Bedingung & Zeit [h] & Umsatz [\%] & $\begin{array}{l}\bar{M}_{\mathrm{n}} \\
\text { [g/mol] }\end{array}$ & $\boldsymbol{\oplus}$ \\
\hline $26-1$ & \multirow{5}{*}{$\begin{array}{c}\text { MMA:RAFT:AIBN } \\
= \\
200: 1: 0,2\end{array}$} & 2 & 17 & 11120 & 1,15 \\
\hline $26-2$ & & 5 & 62 & 16720 & 1,12 \\
\hline $26-3$ & & 9 & 84 & 21300 & 1,1 \\
\hline $26-4$ & & 15 & 93 & 23480 & 1,1 \\
\hline $26-5$ & & 24 & 95 & 23120 & 1,1 \\
\hline
\end{tabular}

Tabelle A-3 Copolymerisation B: MMA (5 mol/L) und CMA (1 mol\%) mit dem RAFT-Agens $(24 \mathrm{mmol} / \mathrm{L})$ und AIBN $(5 \mathrm{mmol} / \mathrm{L})$ in DMF $(10 \mathrm{~mL})$ bei $70^{\circ} \mathrm{C}$.

\begin{tabular}{|c|c|c|c|c|c|}
\hline Probe & Bedingung & Zeit [h] & Umsatz [\%] & $\begin{array}{l}\bar{M}_{\mathrm{n}} \\
{[\mathrm{g} / \mathrm{mol}]}\end{array}$ & $\boldsymbol{\oplus}$ \\
\hline $39-1$ & \multirow{5}{*}{$\begin{array}{c}\text { MMA:RAFT:AIBN } \\
= \\
200: 1: 0,2\end{array}$} & 1,5 & 11 & 10300 & 1,19 \\
\hline $39-2$ & & 3 & 39 & 11120 & 1,2 \\
\hline $39-3$ & & 5 & 62 & 14770 & 1,16 \\
\hline $39-4$ & & 8 & / & / & / \\
\hline $39-5$ & & 8 & 79 & 18350 & 1,13 \\
\hline
\end{tabular}




\begin{tabular}{|l|l|l|l|l|l|}
\hline $\mathbf{3 9 - 6}$ & 14 & 96 & 20728 & 1,12 \\
\hline $\mathbf{3 9 - 7}$ & 24 & 100 & 21582 & 1,12 \\
\hline
\end{tabular}

Tabelle A-4 Homopolymerisation B: CMA (200 mmol/L) mit dem RAFT-Agens $(10 \mathrm{mmol} / \mathrm{L})$ und AIBN $(2 \mathrm{mmol} / \mathrm{L})$ in DMF $(20 \mathrm{~mL})$ bei $70^{\circ} \mathrm{C}$.

\begin{tabular}{|c|c|c|c|c|c|}
\hline Probe & Bedingung & Zeit [h] & Umsatz [\%] & $\begin{array}{l}\bar{M}_{\mathrm{n}} \\
\text { [g/mol] }\end{array}$ & $\boldsymbol{D}$ \\
\hline $58-1$ & \multirow{10}{*}{$\begin{array}{c}\text { CMA:RAFT:AIBN } \\
= \\
20: 1: 0,2\end{array}$} & 0,5 & I & / & / \\
\hline $58-2$ & & 1 & / & / & / \\
\hline $58-3$ & & 2 & 4 & 1152 & 2,14 \\
\hline $58-4$ & & 3 & 8 & / & 1,58 \\
\hline 58-5 & & 4 & 13 & 18350 & 1,13 \\
\hline $58-6$ & & 5,5 & 20 & 20728 & 1,12 \\
\hline 58-7 & & 7 & 22 & 21582 & 1,12 \\
\hline $58-8$ & & 10 & 25 & & \\
\hline 58-9 & & 14 & 22 & & \\
\hline $58-10$ & & 24 & 21 & & \\
\hline
\end{tabular}

Tabelle A-5 Homopolymerisation C: BA (2,8 mol/L) mit dem RAFT-Agens $(13 \mathrm{mmol} / \mathrm{L})$ und AIBN $(3 \mathrm{mmol} / \mathrm{L})$ in DMF $(10 \mathrm{~mL})$ bei $70^{\circ} \mathrm{C}$.

\begin{tabular}{|l|l|l|l|l|l|}
\hline Probe & Bedingung & Zeit [h] & Umsatz [\%] & $\begin{array}{l}\overline{\boldsymbol{M}}_{\mathbf{n}} \\
{[\mathbf{g} / \mathbf{m o l}]}\end{array}$ & $\boldsymbol{D}$ \\
\hline $\mathbf{5 6 - 1}$ & & 0,25 & $/$ & $/$ & $/$ \\
\hline $\mathbf{5 6 - 2}$ & & 0,5 & 10 & 2200 & 1,70 \\
\hline $\mathbf{5 6 - 3}$ & & 1 & 52 & 15900 & 1,25 \\
\hline $\mathbf{5 6 - 4}$ & BA:RAFT:AIBN $=$ & 1,5 & 60 & 17800 & 1,24 \\
\hline $\mathbf{5 6 - 5}$ & $200: 1: 0,2$ & 2 & 76 & 21600 & 1,33 \\
\hline $\mathbf{5 6 - 6}$ & & 3 & 81 & 22800 & 1,37 \\
\hline $\mathbf{5 6 - 7}$ & & 6,5 & 89 & 25500 & 1,47 \\
\hline $\mathbf{5 6 - 8}$ & & 14 & 92 & 24730 & 1,41 \\
\hline
\end{tabular}


Tabelle A-6 Copolymerisation C: BA (3,7 mol/L) und CA $(5 \mathrm{~mol} \%)$ mit dem RAFT-Agens $(19 \mathrm{mmol} / \mathrm{L})$ und AIBN $(4 \mathrm{mmol} / \mathrm{L})$ in DMF $(8,9 \mathrm{~mL})$ bei $70^{\circ} \mathrm{C}$.

\begin{tabular}{|l|l|l|l|l|l|}
\hline Probe & Bedingung & Zeit [h] & Umsatz [\%] & $\begin{array}{l}\overline{\boldsymbol{M}}_{\mathbf{n}} \\
{[\mathbf{g} / \mathbf{m o l}]}\end{array}$ & $\boldsymbol{\Xi}$ \\
\hline $\mathbf{4 2 - 1}$ & & 1 & $/$ & $/$ & $/$ \\
\hline $\mathbf{4 2 - 2}$ & & 3 & $/$ & $/$ & $/$ \\
\hline $\mathbf{4 2 - 3}$ & & 5 & $/$ & $/$ & $/$ \\
\hline $\mathbf{4 2 - 4}$ & & 8 & $/$ & $/$ & $/$ \\
\hline $\mathbf{4 2 - 5}$ & BA:RAFT:AIBN $=$ & 14 & 34 & 15900 & 1,19 \\
\hline $\mathbf{4 2 - 6}$ & $200: 1: 0,2$ & 19 & 46 & 28800 & 1,35 \\
\hline $\mathbf{4 2 - 7}$ & & 38 & 43 & 33300 & 1,61 \\
\hline $\mathbf{4 2 - 8}$ & & 24 & 75 & 30000 & 1,46 \\
\hline $\mathbf{4 2 - 9}$ & & 48 & 38 & 30100 & 1,60 \\
\hline
\end{tabular}

Tabelle A-7 Copolymerisation D: MMA (2,7 mol/L), BA(2,7 mol/L) und HEMA (2 mol\%) mit dem RAFT-Agens $(14 \mathrm{mmol} / \mathrm{L})$ und AIBN $(3 \mathrm{mmol} / \mathrm{L})$ in Toluol $(4 \mathrm{~mL})$ bei $60^{\circ} \mathrm{C}$. Die Probe hat eine Zusammensetzung von $F_{\mathrm{MMA}}=57 \mathrm{~mol} \%, F_{\mathrm{BA}}=37 \mathrm{~mol} \%$ und $F_{\mathrm{HEMA}}=6 \mathrm{~mol} \%$.

\begin{tabular}{|l|l|l|l|l|l|}
\hline Probe & Bedingung & Zeit [h] & Umsatz [\%] & $\begin{array}{l}\overline{\boldsymbol{M}}_{\mathbf{n}} \\
{[\mathbf{g} / \mathbf{m o l}]}\end{array}$ & Ð \\
\hline $\mathbf{1 8 - 7}$ & $\begin{array}{c}\text { MMA:RAFT:AIBN } \\
=\end{array}$ & 6 & 17 & 14800 & 1,36 \\
& $200: 1: 0,2$ & & & & \\
\hline
\end{tabular}

Tabelle A-8 Übersicht für verwendete makroRAFT-Agenzien.

\begin{tabular}{|l|c|l|l|l|l|}
\hline Probe & $\begin{array}{l}\text { Bedingung } \\
\text { MMA:RAFT:AIBN }\end{array}$ & Zeit $[\mathbf{h}]$ & $\begin{array}{l}\text { Umsatz } \\
{[\%]}\end{array}$ & $\begin{array}{l}\overline{\boldsymbol{M}}_{\mathbf{n}} \\
\text { [g/mol] }\end{array}$ & $\boldsymbol{\Xi}$ \\
\hline I & $200: 1: 0,2$ & 5 & 4 & 12000 & 1,20 \\
\hline II & $200: 1: 0,2$ & 5 & 6 & 14300 & 1,19 \\
\hline III & $50: 1: 0,2$ & 5 & 17 & 5800 & 1,18 \\
\hline IV & $500: 1: 0,2$ & 24 & 33 & 47500 & 1,30 \\
\hline
\end{tabular}

Tabelle A-9 Blockcopolymerisation A: BA (4,36 mol/L) mit makroRAFT-Agens I (4,4 mmol/L) und AIBN ( $1 \mathrm{mmol} / \mathrm{L})$ in Toluol $(3 \mathrm{~mL})$ bei $70^{\circ} \mathrm{C}$.

\begin{tabular}{|l|l|l|l|l|l|}
\hline Probe & Bedingung & Zeit [min] & $\begin{array}{l}\text { Umsatz } \\
{[\%]}\end{array}$ & $\begin{array}{l}\overline{\boldsymbol{M}}_{\mathbf{n}} \\
{[\mathbf{g} / \mathbf{m o l}]}\end{array}$ & $\boldsymbol{Ð}$ \\
\hline $\mathbf{3 0 - 1}$ & $\begin{array}{c}\text { BA:makroRAFT:AIBN } \\
=\end{array}$ & 10 & 4 & 21300 & 1,21 \\
\hline $\mathbf{3 0 - 2}$ & 15 & 6 & 31400 & 1,23 \\
\hline
\end{tabular}




\begin{tabular}{|l|l|l|l|l|l|}
\hline $\mathbf{3 0 - 3}$ & $1000: 1: 0,2$ & 20 & 17 & 42000 & 1,23 \\
\hline $\mathbf{3 0 - 4}$ & & & 33 & 64700 & 1,24 \\
\hline $\mathbf{3 0 - 5}$ & & 14 & 51 & 87400 & 1,31 \\
\hline
\end{tabular}

Tabelle A-10 Blockcopolymerisation B: BA (1,78 mol/L) mit makroRAFT-Agens IV (1,7 mmol/L) und AIBN ( $1 \mathrm{mmol} / \mathrm{L})$ in Toluol $(13 \mathrm{~mL})$ bei $70^{\circ} \mathrm{C}$.

\begin{tabular}{|l|l|l|l|l|l|}
\hline Probe & Bedingung & Zeit $[\mathbf{h}]$ & $\begin{array}{l}\text { Umsatz } \\
{[\mathbf{\%}]}\end{array}$ & $\begin{array}{l}\overline{\boldsymbol{M}}_{\mathbf{n}} \\
\mathbf{[ g / m o l ]}\end{array}$ & $\boldsymbol{\Xi}$ \\
\hline $\mathbf{3 7 - 6}$ & & 0,5 & 5 & 86500 & 1,37 \\
\hline $\mathbf{3 7 - 7}$ & $\begin{array}{c}\text { BA:makroRAFT:AIBN } \\
=\end{array}$ & 0,75 & 10 & 93400 & 1,41 \\
\hline $\mathbf{3 7 - 8}$ & $1000: 1: 0,2$ & 1 & 16 & 98500 & 1,65 \\
\hline $\mathbf{3 7 - 1 0}$ & & 2 & 29 & 106000 & 1,61 \\
\hline
\end{tabular}

Tabelle A-11 Blockcopolymerisation C: BA (4,36 mol/L) mit makroRAFT-Agens III $(4,4 \mathrm{mmol} / \mathrm{L})$ und AIBN (1 mmol/L) in Toluol $(3 \mathrm{~mL})$ bei $70^{\circ} \mathrm{C}$.

\begin{tabular}{|c|c|c|c|c|c|}
\hline Probe & Bedingung & Zeit [min] & $\begin{array}{l}\text { Umsatz } \\
\text { [\%] }\end{array}$ & $\begin{array}{l}\bar{M}_{\mathrm{n}} \\
\text { [g/mol] }\end{array}$ & $\boldsymbol{\oplus}$ \\
\hline $55-1$ & \multirow{3}{*}{$\begin{array}{c}\text { BA:makroRAFT:AIBN } \\
= \\
1000: 1: 0,2\end{array}$} & 10 & 10 & 22200 & 1,39 \\
\hline $55-2$ & & 20 & 33 & 53500 & 1,28 \\
\hline $55-3$ & & 60 & 61 & 95100 & 1,45 \\
\hline
\end{tabular}

Tabelle A-12 Blockcopolymerisation D: BMA (3,5 mol/L) mit makroRAFT-Agens II $(3,5 \mathrm{mmol} / \mathrm{L})$ und AIBN (1 mmol/L) in Toluol $(8 \mathrm{~mL})$ bei $70^{\circ} \mathrm{C}$.

\begin{tabular}{|c|c|c|c|c|c|}
\hline Probe & Bedingung & Zeit [h] & $\begin{array}{l}\text { Umsatz } \\
\text { [\%] }\end{array}$ & $\begin{array}{l}\bar{M}_{\mathrm{n}} \\
{[\mathrm{g} / \mathrm{mol}]}\end{array}$ & $\boldsymbol{D}$ \\
\hline $49-3$ & \multirow{3}{*}{$\begin{array}{c}\text { BA:makroRAFT:AIBN } \\
= \\
1000: 1: 0,2\end{array}$} & 1 & 14 & 40600 & 1,41 \\
\hline $49-6$ & & 4 & 44 & 94200 & 1,35 \\
\hline 49-10 & & 29 & 90 & 100200 & 1,67 \\
\hline
\end{tabular}

Tabelle A-13 Blockcopolymerisation ohne Coumarin-Monomer: a) BA (2 mol/L) mit makroRAFTAgens $(4,6 \mathrm{mmol} / \mathrm{L})$ und AIBN $(1 \mathrm{mmol} / \mathrm{L})$ in Toluol $(4 \mathrm{~mL})$ bei $70^{\circ} \mathrm{C}$.; b) BA $(4 \mathrm{~mol} / \mathrm{L})$ mit makroRAFT-Agens $(4 \mathrm{mmol} / \mathrm{L})$ und AIBN $(1 \mathrm{mmol} / \mathrm{L})$ in Toluol $(10 \mathrm{~mL})$ bei $70^{\circ} \mathrm{C}$.

\begin{tabular}{|c|c|l|l|l|l|}
\hline Probe & $\begin{array}{l}\text { Bedingung } \\
\text { BA:makroRAFT:AIBN }\end{array}$ & Zeit [h] & $\begin{array}{l}\text { Umsatz } \\
{[\%]}\end{array}$ & $\begin{array}{l}\overline{\boldsymbol{M}}_{\mathbf{n}} \\
{[\mathbf{g} / \mathbf{m o l}]}\end{array}$ & $\boldsymbol{Ð}$ \\
\hline a) $\mathbf{1 0 - 9}$ & $1000: 1: 0,2$ & 1 & 47 & 78300 & 1,22 \\
\hline b) $\mathbf{2 3 - 4}$ & $500: 1: 0,2$ & 0,75 & 18 & 43000 & 1,33 \\
\hline
\end{tabular}




\section{Anhang B}
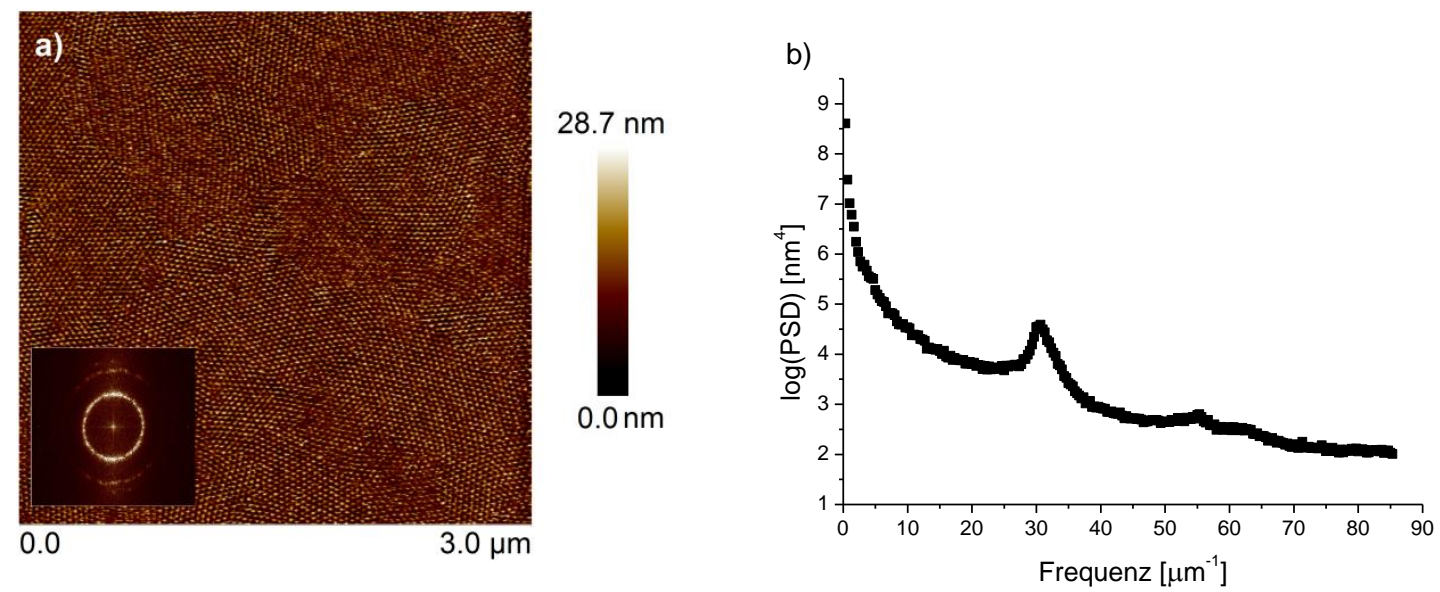

Abbildung B-1 Bestimmung des Domänenabstands mittels PSD-Funktion: a) AFM-Höhenprofil von Probe VIII, wobei der Ausschnitt die FFT zeigt. Cantilever: FESPA. PF setpoint automatisch gesetzt. Auflösung jew. 512 x 512 Pixel. b) zweidimensionale, isotropische PSD-Verteilung des Höhenprofils.
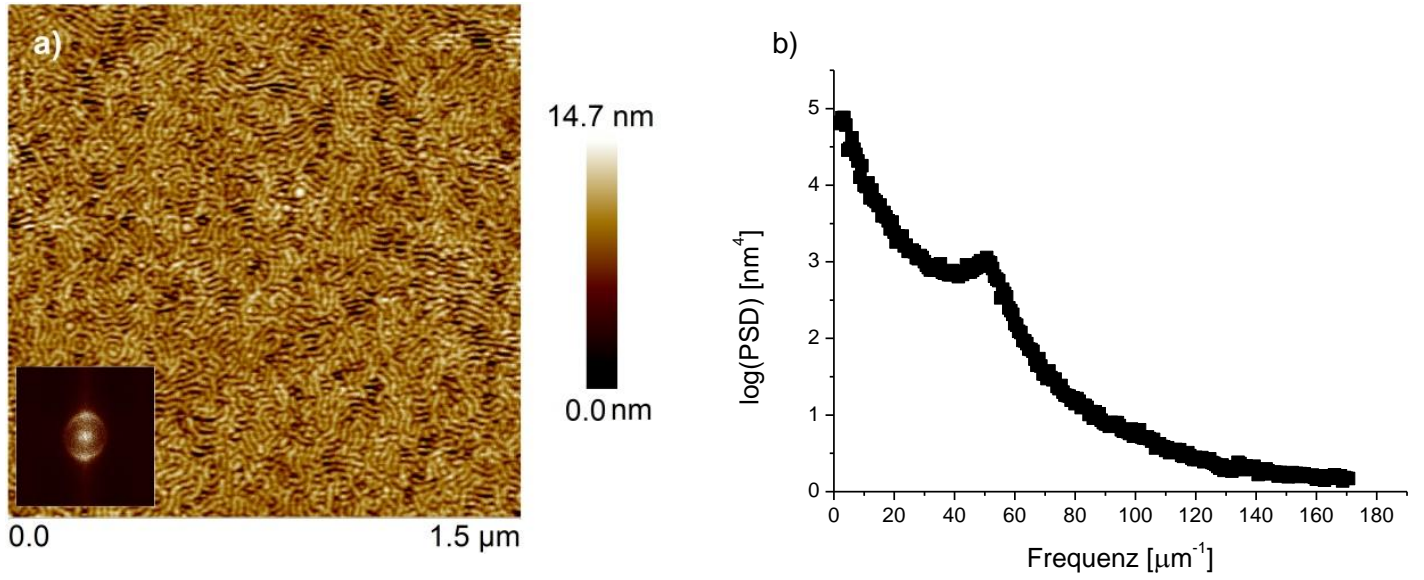

Abbildung B-2 Bestimmung des Domänenabstands mittels PSD-Funktion: a) AFM-Höhenprofil von Probe $\mathbf{X}$, wobei der Ausschnitt die FFT zeigt. Cantilever: FESPA. PF setpoint automatisch gesetzt. Auflösung jew. 512 x 512 Pixel. b) zweidimensionale, isotropische PSD-Verteilung des Höhenprofils. 

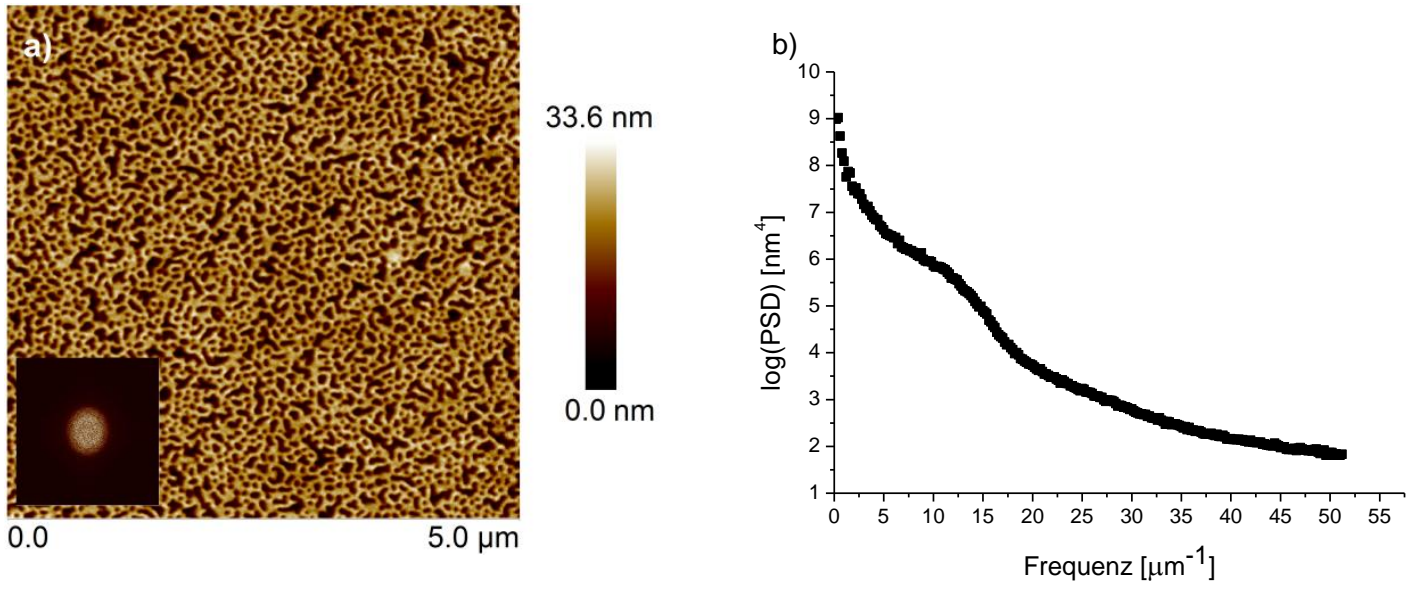

Abbildung B-3 Bestimmung des Domänenabstands mittels PSD-Funktion: a) AFM-Höhenprofil von Probe V, wobei der Ausschnitt die FFT zeigt. Cantilever: FESPA. PF setpoint automatisch gesetzt. Auflösung jew. 512 x 512 Pixel. b) zweidimensionale, isotropische PSD-Verteilung des Höhenprofils.
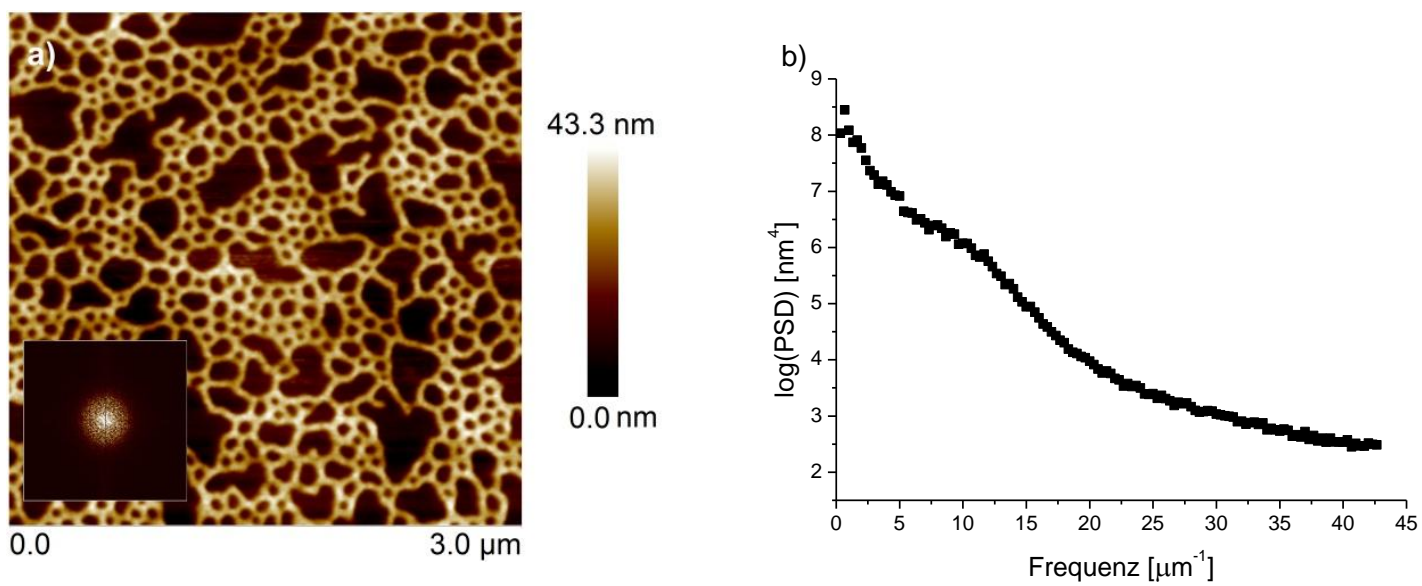

Abbildung B-4 Bestimmung des Domänenabstands mittels PSD-Funktion: a) AFM-Höhenprofil von Probe VI, wobei der Ausschnitt die FFT zeigt. Cantilever: FESPA. PF setpoint automatisch gesetzt. Auflösung jew. 512 x 512 Pixel. b) zweidimensionale, isotropische PSD-Verteilung des Höhenprofils. 

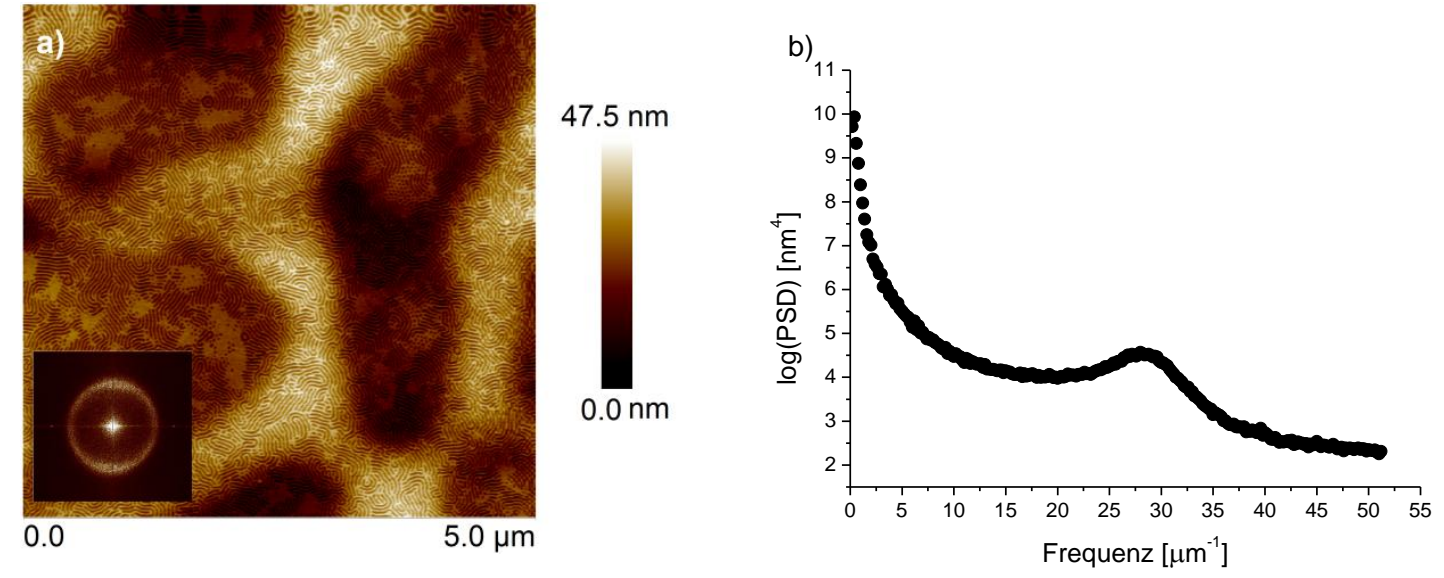

Abbildung B-5 Bestimmung des Domänenabstands mittels PSD-Funktion: a) AFM-Höhenprofil von Probe XI, wobei der Ausschnitt die FFT zeigt. Cantilever: FESPA. PF setpoint automatisch gesetzt. Auflösung jew. 512 x 512 Pixel. b) zweidimensionale, isotropische PSD-Verteilung des Höhenprofils.
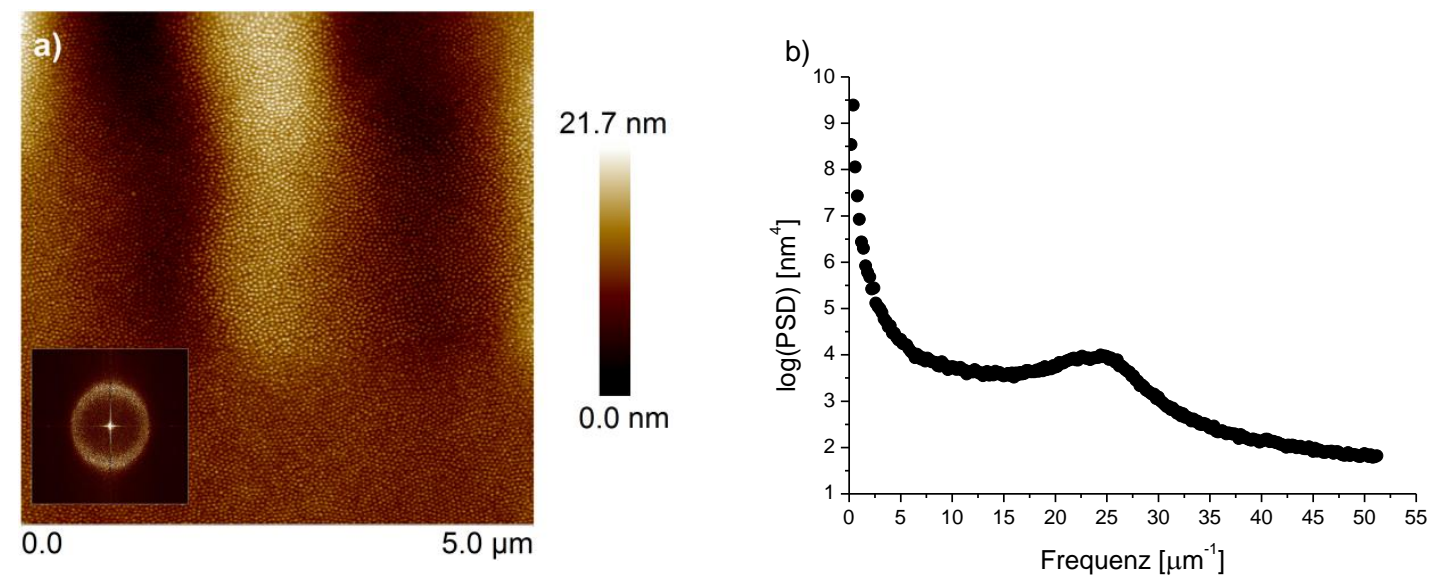

Abbildung B-6 Bestimmung des Domänenabstands mittels PSD-Funktion: a) AFM-Höhenprofil von Probe XII, wobei der Ausschnitt die FFT zeigt. Cantilever: FESPA. PF setpoint automatisch gesetzt. Auflösung jew. 512 x 512 Pixel. b) zweidimensionale, isotropische $P S D$-Verteilung des Höhenprofils. 


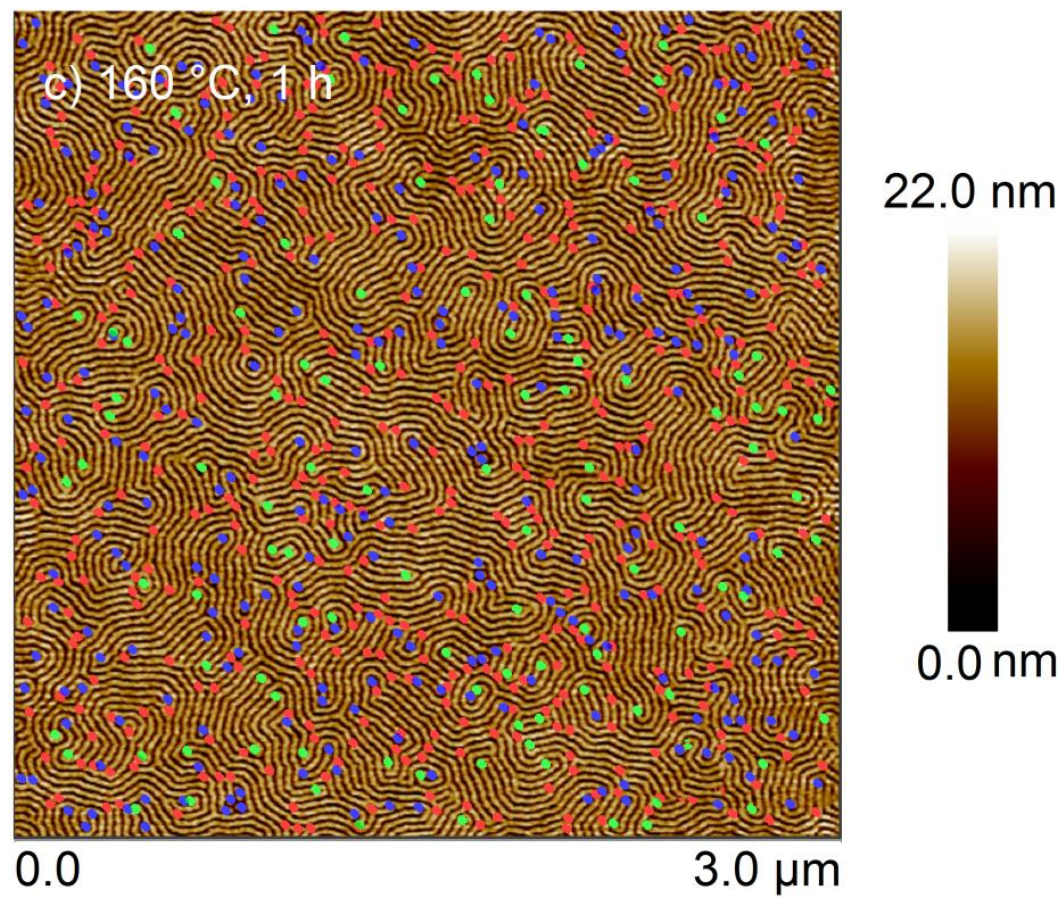

Abbildung B-7 Ausgezähltes AFM-Höhenprofil des BCPs VII nach $1 \mathrm{~h}$ Tempern bei $160^{\circ} \mathrm{C}$, wobei die Defekte Kurven (grün), Sackgassen (blau) und Kreuzungen (rot) bestimmt wurden. Cantilever: ScanAsystAir. PF setpoint manuell festgelegt. Auflösung 512 x 512 Pixel.

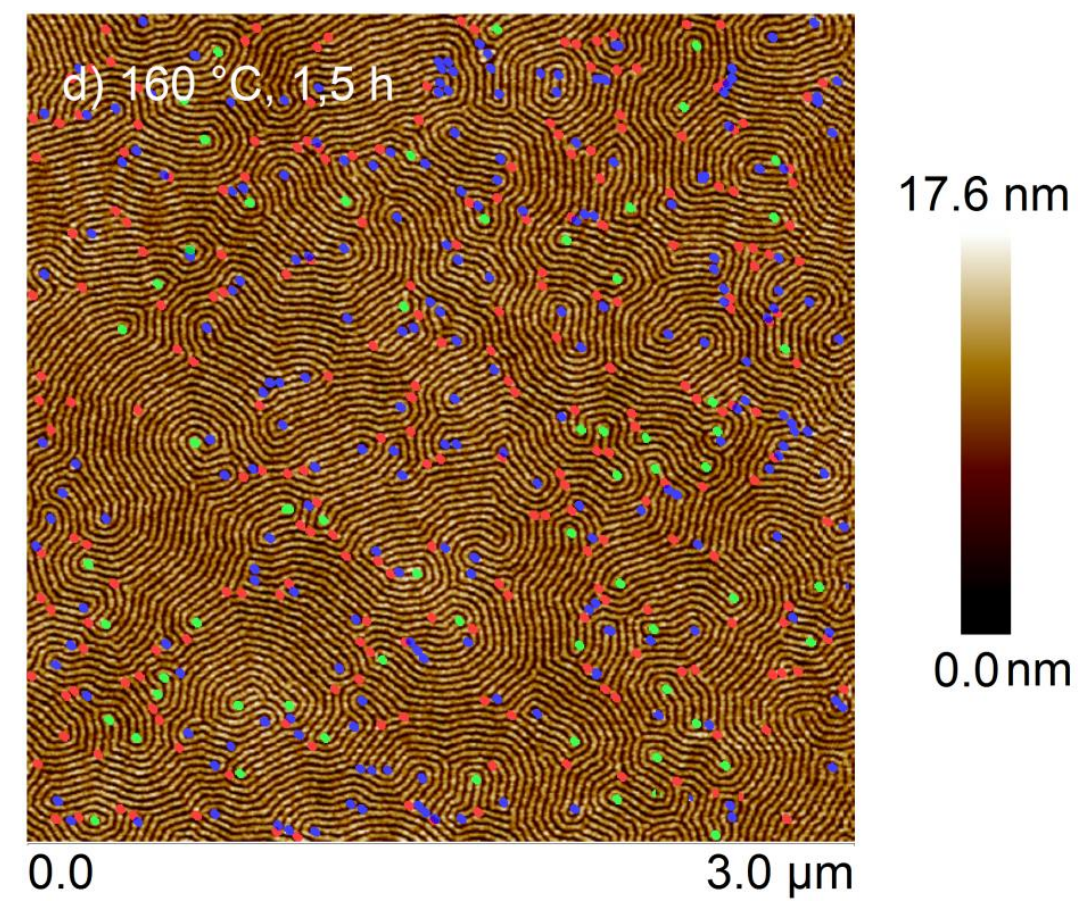

Abbildung B-8 Ausgezähltes AFM-Höhenprofil des BCPs VII nach 1,5 h Tempern bei $160{ }^{\circ} \mathrm{C}$, wobei die Defekte Kurven (grün), Sackgassen (blau) und Kreuzungen (rot) bestimmt wurden. Cantilever: ScanAsystAir. PF setpoint manuell festgelegt. Auflösung 512 x 512 Pixel. 


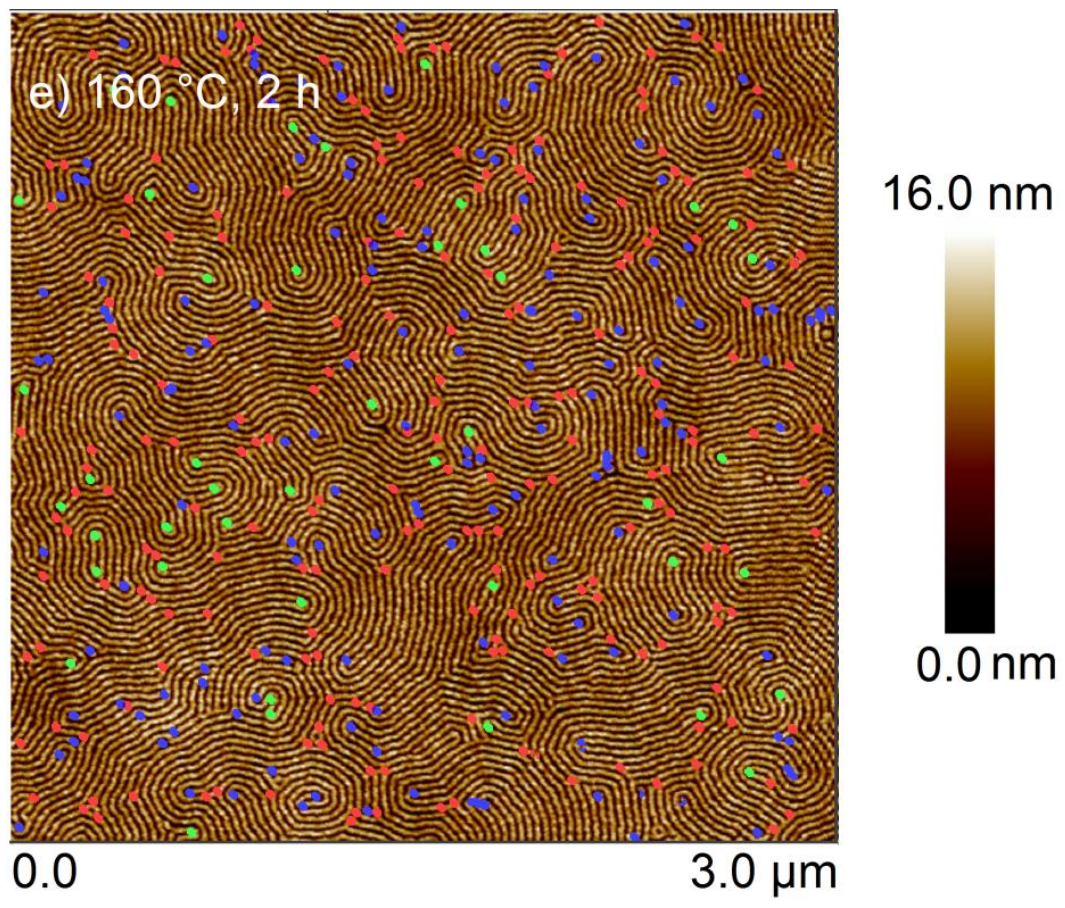

Abbildung B-9 Ausgezähltes AFM-Höhenprofil des BCPs VII nach $2 \mathrm{~h}$ Tempern bei $160^{\circ} \mathrm{C}$, wobei die Defekte Kurven (grün), Sackgassen (blau) und Kreuzungen (rot) bestimmt wurden. Cantilever: ScanAsystAir. PF setpoint manuell festgelegt. Auflösung 512 x 512 Pixel.

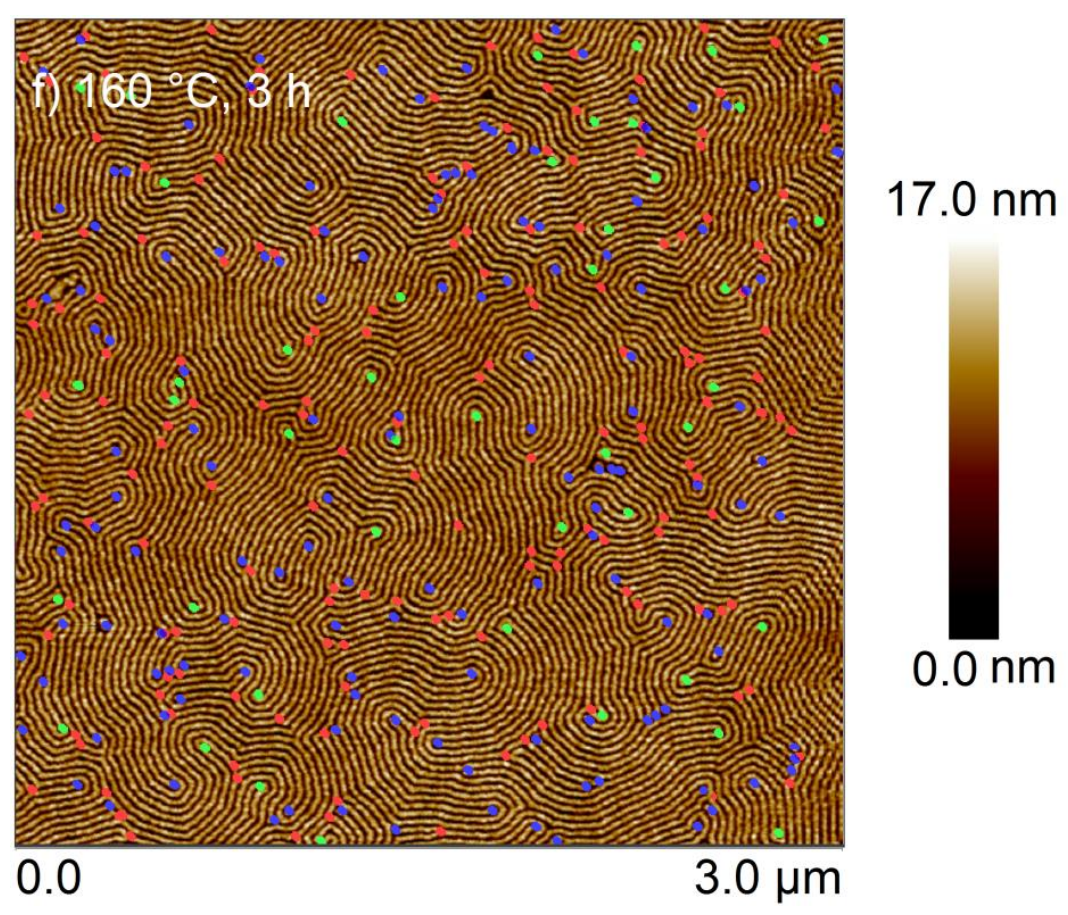

Abbildung B-10 Ausgezähltes AFM-Höhenprofil des BCPs VII nach $3 \mathrm{~h}$ Tempern bei $160^{\circ} \mathrm{C}$, wobei die Defekte Kurven (grün), Sackgassen (blau) und Kreuzungen (rot) bestimmt wurden. Cantilever: ScanAsystAir. PF setpoint manuell festgelegt. Auflösung 512 x 512 Pixel. 


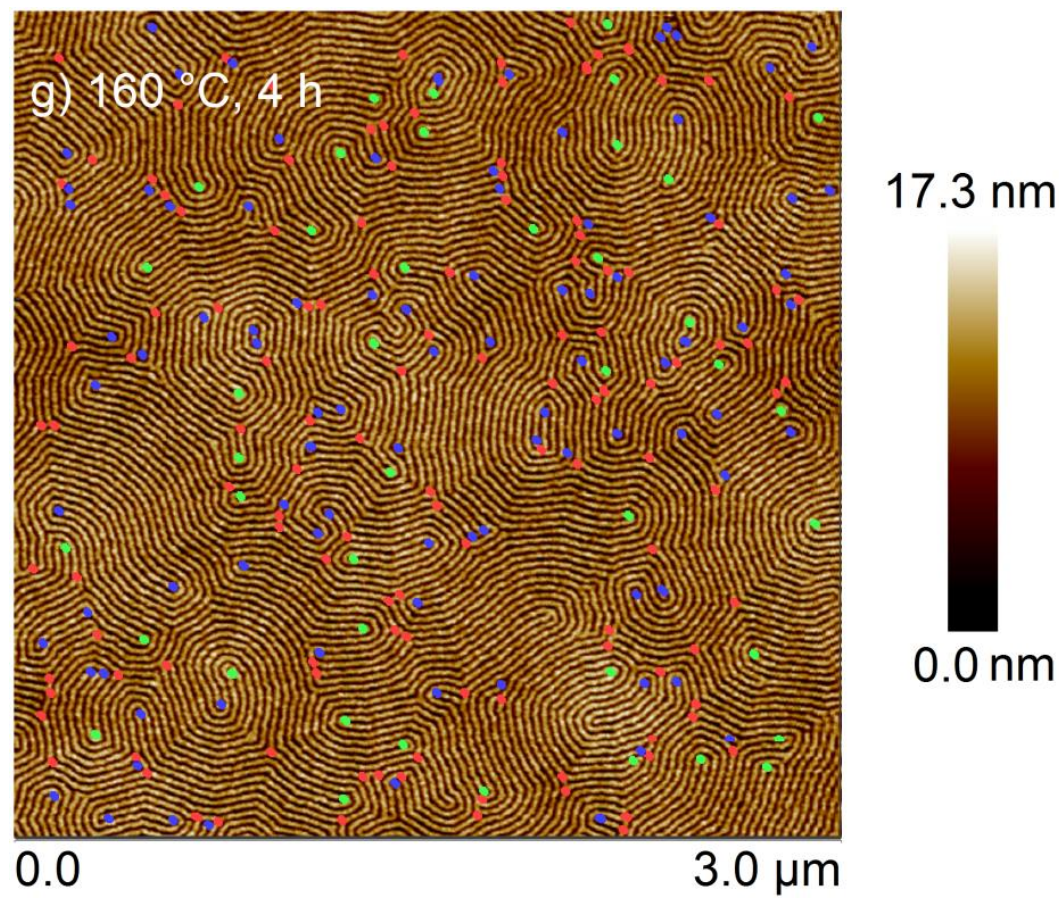

Abbildung B-11 Ausgezähltes AFM-Höhenprofil des BCPs VII nach $4 \mathrm{~h}$ Tempern bei $160^{\circ} \mathrm{C}$, wobei die Defekte Kurven (grün), Sackgassen (blau) und Kreuzungen (rot) bestimmt wurden. Cantilever: ScanAsystAir. PF setpoint manuell festgelegt. Auflösung 512 x 512 Pixel.

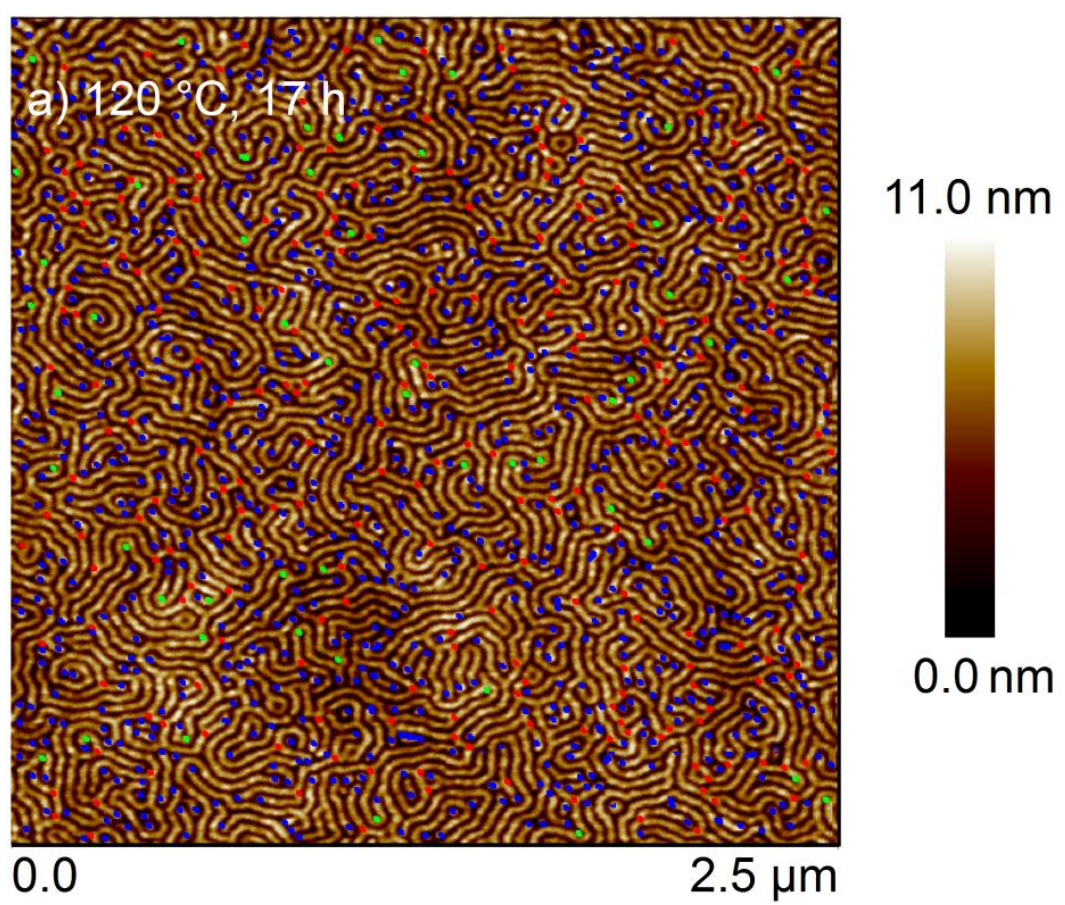

Abbildung B-12 Ausgezähltes AFM-Höhenprofil des BCPs VII nach $17 \mathrm{~h}$ Tempern bei $120^{\circ} \mathrm{C}$, wobei die Defekte Kurven (grün), Sackgassen (blau) und Kreuzungen (rot) bestimmt wurden. Cantilever: RFESP. PF setpoint manuell festgelegt. Auflösung 512 x 512 Pixel. 


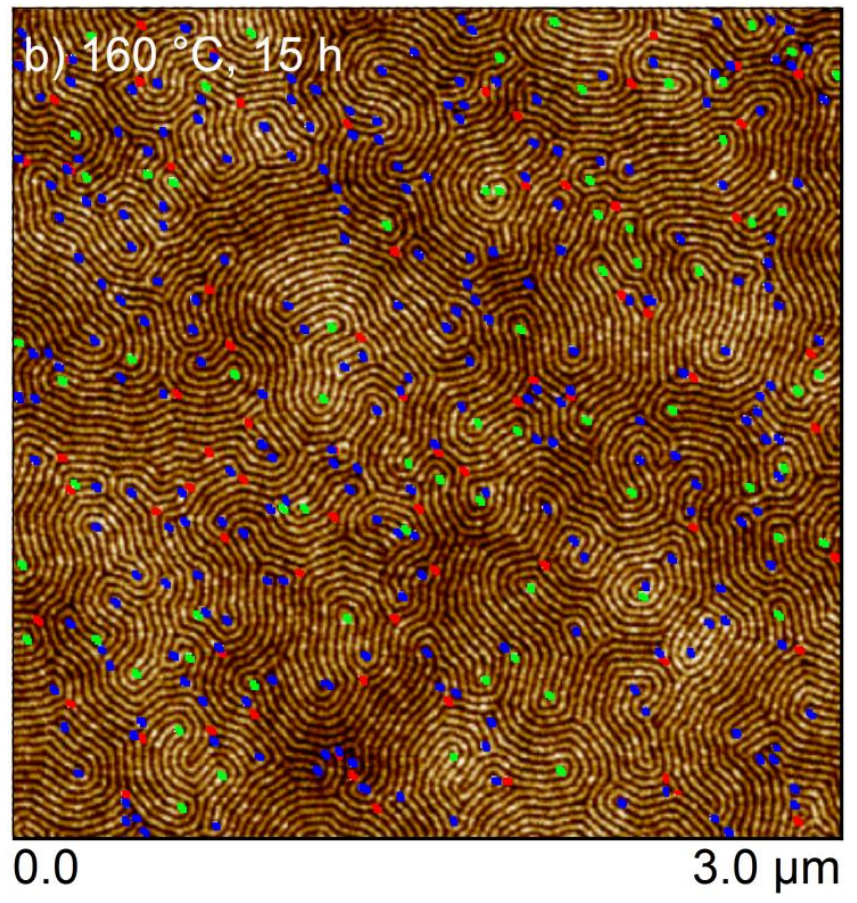

$8.7 \mathrm{~nm}$

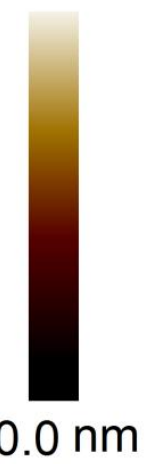

Abbildung B-13 Ausgezähltes AFM-Höhenprofil des BCPs VII nach $15 \mathrm{~h}$ Tempern bei $160^{\circ} \mathrm{C}$, wobei die Defekte Kurven (grün), Sackgassen (blau) und Kreuzungen (rot) bestimmt wurden. Cantilever: ScanAsystAir. PF setpoint manuell festgelegt. Auflösung 512 x 512 Pixel.

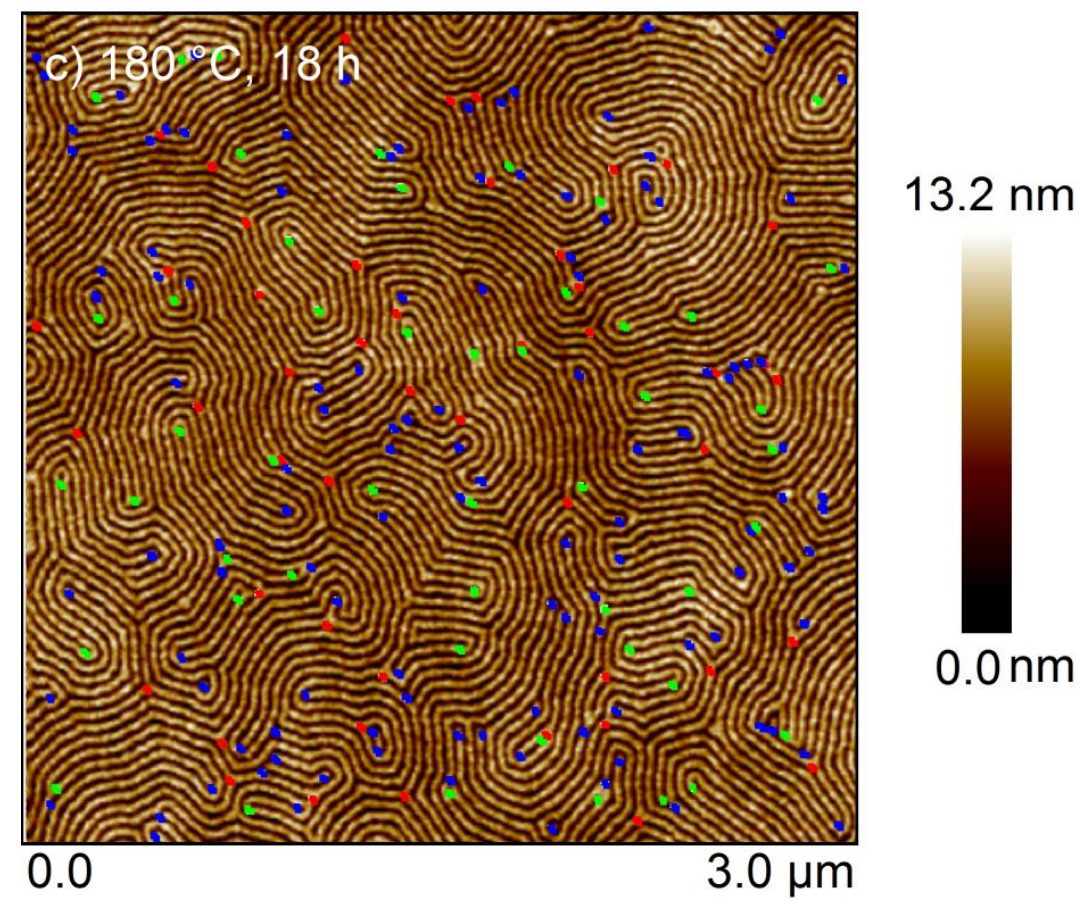

Abbildung B-14 Ausgezähltes AFM-Höhenprofil des BCPs VII nach $18 \mathrm{~h}$ Tempern bei $180^{\circ} \mathrm{C}$, wobei die Defekte Kurven (grün), Sackgassen (blau) und Kreuzungen (rot) bestimmt wurden. Cantilever: RFESP. PF setpoint manuell festgelegt. Auflösung 512 x 512 Pixel. 


\section{Anhang C}

\begin{tabular}{|c|c|}
\hline Abkürzung & Name \\
\hline Äq. & Äquivalent \\
\hline AFM & Rasterkraftmikroskop (engl: Atomic Force Microscopy) \\
\hline AIBN & 2,2'-Azo-bis-(iso-butyronitril) \\
\hline ATRP & Atom Transfer Radical Polymerization \\
\hline \multirow[t]{2}{*}{ ATRP } & Atomtransfer-radikalische Polymerisation (engl: Atom- \\
\hline & Transfer Radical Polymerization) \\
\hline BA & n-Butylacrylat (engl: $n$-Butylacrylate) \\
\hline $\mathrm{BCP}$ & Blockcopolymer \\
\hline BMA & Butylmethacrylat (engl: Butyl Methacrylate) \\
\hline bzw. & Beziehungsweise \\
\hline CA & 7-Acryloyloxyethoxy-4-methylcoumarin \\
\hline CMA & 7-(2-Methacryloyloxyethoxy)-4-methylcoumarin \\
\hline CTA & Chain Transfer Agent \\
\hline \multirow[t]{2}{*}{ DMA } & Dynamisch-mechanische Analyse (engl: Dynamic \\
\hline & Mechanical Analysis) \\
\hline DMF & Dimethylformamid \\
\hline DMT & Dejarguin-Muller-Toporov \\
\hline et al. & et alii (und andere) \\
\hline Gew.\% & Gewichtsprozent \\
\hline GPC & Gel-Permeations-Chromatographie \\
\hline HEMA & Hydroxyethylmethacrylat \\
\hline $\mathrm{K}$ & Kelvin \\
\hline makroRAFT & makromolekulares RAFT-Agens \\
\hline MMA & Methylmethacrylat (engl: Methyl Methacrylate) \\
\hline MMV & Molmassenverteilung \\
\hline $\mathrm{mol} \%$ & Molprozent \\
\hline MPS & Mikrophasenseparation (engl: Micro-Phase Separation) \\
\hline NMP & Nitroxidvermittelte Polymerisation (engl: Nitroxide- \\
\hline & Mediated Polymerization) \\
\hline NMR & Kernspinresonanz (engl: Nuclear Magnetic Resonance) \\
\hline ODT & $\begin{array}{l}\text { Ordnungs-Unordnungs-Übergang (engl.: order-disorder } \\
\text { transition) }\end{array}$ \\
\hline
\end{tabular}


$P$

PD

$\mathrm{PF}$

PSD

QNM

$\mathrm{R}$

RAFT

RDRP

RI

RT

sog.

TGA

THF

UV

Vis

Z

z. B.

\section{Symbole}

[...]

$[\eta]$

A

A

c

D

$d$

D

E

$E_{r}$

$f$

F

$G_{\mathrm{m}}$

$H_{\mathrm{m}}$

I poly

Photodimerisierung

PeakForce

Leistungsspektraldichte (engl.: Power Spectral Density)

Quantitative Nanomechanical Mapping

Abgangsgruppe, Radikal

Reversibler Additions-Fragmentierungs-Kettentransfer

(engl: Reversible Addition-Fragmentation chain

Transfer)

Reversibel-Desaktivierte Radikalische Polymerisation

Brechungsindex

Raumtemperatur

sogenannt

Thermogravimetrische Analyse

Tetrahydrofuran

ultraviolett

sichtbar (engl.: visible)

stabilisierende Gruppe

zum Beispiel

Name

Konzentration der benannten Komponente

intrinsische Viskosität

Absorption

Mark-Houwink-Parameter

Konzentraion

Domänenabstand

Relaxationszeit

Dispersität

Elastizitätsmodul

reduziertes Elastizitätsmodul

Frequenz

Kraft

Gibbs-Energie

Mischungsenthalpie

Initiator 
K

k

$k_{\text {B }}$

$k_{\mathrm{C}}$

$k_{\mathrm{d}}$

$k_{\mathrm{p}}$

$k_{\mathrm{st}}$

$k_{\mathrm{t}}$

$k_{\text {tr }}$

$K_{\text {tr }}$

$L$

m

M

$\bar{M}_{\mathrm{n}}$

$\bar{M}_{\mathrm{w}}$

$\bar{M}_{\mathrm{n}}$ theor

$n$

N

$n$

$N_{\text {A }}$

$\bar{P}_{\mathrm{n}}$

$R$

$R$

$r_{\mathrm{d}}$

$r_{\mathrm{i}}$

$R_{\mathrm{p}}$

$r_{\mathrm{p}}$

$r_{\mathrm{t}}$

$S_{\mathrm{D}}$

$S_{\mathrm{m}}$

$T$

$t$

$T_{\mathrm{g}}$

U

V

$V_{\mathrm{h}}$

X
Mark-Houwink-Parameter

Wellenzahl

Boltzmannkonstante

Federkonstante

Geschwindigkeitskoeffizient des Initiatorzerfalls

Geschwindigkeitskoeffizient der Wachstumsreaktion

Geschwindigkeitskoeffizient der Startreaktion

Geschwindigkeitskoeffizient der Abbruchreaktionen

Gechwindigkeitskoeffizient der Transferreaktion

Transferkonstante

Gesamtmessstrecke des Höhenprofils

Masse

molare Masse

Zahlenmittel der Molmasse

Gewichtsmittel der Molmasse

theortische Molmasse

Anzahl

Polymerisationsgrad

Stoffmenge

Avogadro-Konstante

Polymerisationsgrad

ideale Gaskonstante

Spitzenradius

Geschwindigkeit der Zerfallsreaktion des Initiators

Copolymerisationsparameter

Bruttopolymerisationsgeschwindigkeit

Geschwindigkeit der Propagation

Geschwindigkeit der Terminierung

Deflection Sensitivity

Mischungsentropie

Temperatur

Zeit

Glasübergangstemperatur

Spannung

Volumen

hydrodynamische Volumen

Monomerumsatz 
$Z$

$\delta$

$\varepsilon_{\mathrm{l}}$

$\lambda$

$\lambda_{\mathrm{G}}$

$v$

$\chi$

$\Phi_{\mathrm{i}}$
Auslenkung Cantilever

Cantileververbiegung

Wechselwirkungsenergie

Wellenlänge

Grenzflächendicke

Poissonzahl

Entmischungsparameter (Flory-Huggins-Parameter)

Volumenbruch 


\section{Danksagung}

Mein besonderer Dank gilt Prof. Dr. Philipp Vana für die Möglichkeit, diese interessante und herausfordernde Themenstellung in meiner Dissertation zu bearbeiten. Weiterhin möchte ich ihm für die hilfreichen und motivierenden Diskussionen sowie für seine Unterstützung danken. Prof. Dr. Michael Buback möchte ich für die Übernahme des Koreferats dieser Arbeit danken.

Für die exzellente Kooperation danke ich meinen Projektpartnern des DFG-Projekts „SFB 937“ Prof. Dr. Konrad Samwer, Prof. Dr. Annette Zippelius, Stefanie Finkhäuser und Dr. Alice von der Heydt. Meinen tiefen Dank möchte ich Stefanie Finkhäuser für die vielen DMA-Messungen im Rahmen des SFB-Projekts, für die hilfreichen Diskussionen und die fruchtbare Zusammenarbeit aussprechen. Der Deutschen Forschungsgemeinschaft danke ich für die langjährige finanzielle Unterstützung des Projekts.

Allen Mitgliedern der Arbeitskreise Makromolekulare Chemie sowie Technische und Makromolekulare Chemie danke ich für das entspannte Arbeitsklima und die sehr gute Zusammenarbeit innerhalb der Abteilungen, die wesentlich zur Entstehung dieser Arbeit beigetragen hat. Dr. Hans-Peter Vögele danke ich für technische und theoretische Hilfestellungen. Heike Rohmann und Ingrid Jünke gebührt ein herzlicher Dank für die angenehme Arbeitsatmosphäre und Unterstützung bei den Praktikumsbetreuungen.

Weiterhin danke ich den Mitarbeitern der NMR-Abteilung des Instituts für Organische Chemie für das Messen meiner NMR-Spektren und ebenso den Mitarbeiterinnen des Analytischen Labors in der Anorganischen Chemie, Angelika Wraage, Diana Kumpart und Susanne Petrich, für die durchgeführten Elementaranalysen. Ich danke Dr. Holm Frauendorf und seinem Team für das Messen der Massenspektren. Der Glaswerkstatt möchte ich für zahlreiche Reparaturen und der Mechanik-Werkstatt für den Bau der Probenhalterung für das UV/Vis-Spektrometer danken. Norbert Neisen danke ich für die fürsorgliche Pflege und Wartung meines Computers.

Ich danke Dr. Florian Ehlers für die Einweisung am AFM, Dr. Cathrin Conrad für die am UV Cary und Sebastian Primpke sowie Sandra Lotze für die Unterstützung im Umgang mit den GPC-Anlagen. Großer Dank gilt Vanessa Koch für die kompetente Unterstützung und stete Hilfsbereitschaft bei der Handhabung mit dem AFM. Weiterhin möchte ich mich bei meinen Bachelorstudenten Jens Hagenow und Holger Deutges bedanken, die ich im Laufe meiner Promotion betreuen durfte. Besonders Holger hat durch seinen ausdauernden Einsatz am AFM zum Fortkommen meiner Forschung beigetragen. 
Ein herzlicher Dank geht an meine Korrekturleser Dr. Cathrin Conrad, Dennis Hübner, Michael Hendrich, Vanessa Koch, Hendrik Kattner, Julia Möhrke, Björn Sölter und JanHendrik Schütz für die hilfreiche Kritik und beim Lesen geopferte Zeit. Bei meinen zahlreichen Bürokollegen Dr. Wibke Meiser, Dr. Joachim Morick, Dr. Bastian Ebeling, Hendrik Kattner, Vanessa Koch, Dr. Cathrin Conrad, Dr. Nadja Förster, Sebastian Heise, Jens Hagenow, Jana Rohde und Kamil Kiepura möchte ich mich für viele lustige Diskussionen und die entspannte Büroatmosphäre bedanken.

Spezieller Dank gebührt meinen Kartenspielerinnen Vanessa, Cathrin und Nadja für die vielen Canasta- und Rommé-Runden während der gemütlichen Mittagspausen im Brain sowie die Verkostung gewagter bis schlussendlich kulinarischer Gerichte.

All meinen Freunden danke ich für die schöne und erlebnisreiche Zeit im und neben dem Studium, für die stete Unterstützung in allen Lebensbereichen und die nicht zu vergessenen aufmunternden Worte.

Mein größter Dank gilt meiner Familie, die mich in all meinen Entscheidungen unterstützt und gefördert hat. Insbesondere danke ich meinen Großeltern für ihre Hilfsbereitschaft in jeder Lebenslage. Meinem Bruder Sebastian, meiner Schwägerin Steffi und meiner Nichte Josi danke ich für viele schöne Wochenenden in Mainz, an denen sie mich immer zum Lachen brachten und jeden Stress vergessen ließen. Mein ganz besonderer Dank gebührt meinen Eltern, die in jeglicher Hinsicht die Grundsteine für meinen Weg gelegt und immer an mich geglaubt haben. Ohne euch hätte ich das nicht geschafft! 
